\title{
Jahrbuch der berufs- und wirtschaftspädagogischen Forschung 2017
}

Jürgen Seifried, Susan Seeber, Birgit Ziegler (Hrsg.)

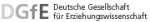


Schriftenreihe der Sektion

Berufs- und Wirtschaftspädagogik

der Deutschen Gesellschaft

für Erziehungswissenschaft (DGfE) 
Jürgen Seifried

Susan Seeber

Birgit Ziegler (Hrsg.)

\section{Jahrbuch der berufs- und} wirtschaftspädagogischen Forschung 2017

Verlag Barbara Budrich

Opladen • Berlin • Toronto 2017 
Bibliografische Information der Deutschen Nationalbibliothek

Die Deutsche Nationalbibliothek verzeichnet diese Publikation in der Deutschen

Nationalbibliografie; detaillierte bibliografische Daten sind im Internet über

http://dnb.d-nb.de abrufbar.

Gedruckt auf säurefreiem und alterungsbeständigem Papier

Alle Rechte vorbehalten

(C) 2017 Verlag Barbara Budrich, Opladen, Berlin \& Toronto

www.budrich-verlag.de

ISBN 978-3-8474-2141-2 (Paperback)

eISBN 978-3-8474-1131-4 (eBook)

Das Werk einschließlich aller seiner Teile ist urheberrechtlich geschützt. Jede Verwertung außerhalb der engen Grenzen des Urheberrechtsgesetzes ist ohne Zustimmung des Verlages unzulässig und strafbar. Das gilt insbesondere für Vervielfältigungen, Übersetzungen, Mikroverfilmungen und die Einspeicherung und Verarbeitung in elektronischen Systemen.

Umschlaggestaltung: Bettina Lehfeldt, Kleinmachnow - www.lehfeldtgraphic.de Typographisches Lektorat: Anja Borkam, Jena 


\section{Inhaltsverzeichnis}

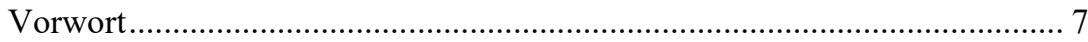

\section{Teil I: Überlegungen zum disziplinären Selbstverständis der Berufs- und Wirtschaftspädagogik}

Karin Büchter

Zum Gehalt berufs- und wirtschaftspädagogischer

Selbstthematisierungen - Rückblick und Ausblick

Matthias Söll

Orientierungspotenziale des Basiscurriculums der Berufs- und

Wirtschaftspädagogik für die disziplinäre Binnen- und Außenlegitimität.... 29

\section{Teil II: Berufliche Lehr-Lern- und Unterrichtsforschung}

Christoph Helm, Jacqueline Netzthaler und Bettina Kreuzer

Kooperatives Lernen im kaufmännischen Unterricht. Eine

Netzwerkanalyse zu sozial-konstruktivistischen Lerntheorien 43

Manuela Niethammer und Anke Langner

Inklusion als fachdidaktischer Anspruch

Svenja Ohlemann und Katja Driesel-Lange

Individuelle Begleitung beruflicher Entwicklung: Kompetenzförderung anhand von Lernstilen.

Mandy Hommel, Bärbel Fürstenau, Claudia Leopold, Héctor Ponce und Mario López

Beitrag von Banken-Webseiten zur Entwicklung der Finanzkompetenz

potentieller Darlehensnehmer/innen über Baufinanzierungen

\section{Teil III: Hochschul- und Lehrerbildungsforschung}

Silke Lange und Dietmar Frommberger

Zur Ausgestaltung schulischer Praxisphasen im beruflichen

Lehramtsstudium - Ergebnisse einer ersten Analyse. 
Heike Jahncke und Karina Kiepe

Handlungsempfehlungen aus dem Einsatz und der Evaluation eines

Tagungsportfolios im Rahmen der Lehrerbildung

Julia Warwas und Andreas Rausch

Unterrichtliche Überzeugungen und Praktiken von Lehrkräften an

Beruflichen Oberschulen - eine fächervergleichende Analyse....

Christian Schmidt

Die Öffnung des Hochschulzugangs für beruflich Gebildete: Förderung studienrelevanter Schlüsselkompetenzen in der Studieneingangsphase am Beispiel der Universität Kassel

\section{Teil IV: Schulentwicklungsforschung}

Marc Casper, Bernadette Dilger, Frederik Fischer, Katharina Fütterer, Nicole Naeve-Stoß und Tade Tramm

Entwicklung beruflicher Schulen im regionalen Verbund....

Herausgeberschaft.

Autorinnen und Autoren 


\section{Vorwort}

Mit Blick auf die thematische und methodische Vielfalt der deutschsprachigen Berufsbildungsforschung - diese bearbeitet z. B. Fragen der Didaktik und Methodik des beruflichen Lernens, der Kompetenzmodellierung und Kompetenzmessung, der Lehrerbildungsforschung, der beruflichen Aus- und Weiterbildung, der Hochschuldidaktik sowie der historischen Berufsbildungsforschung - entschloss sich die Sektion für Berufs- und Wirtschaftspädagogik der Deutschen Gesellschaft für Erziehungswissenschaft (DGfE) im Jahr 2012, ein „Jahrbuch der berufs- und wirtschaftspädagogischen Forschung" herauszugeben. Damit soll der Breite und Tiefe der Forschung und Theoriebildung in der Berufs- und Wirtschaftspädagogik besser Rechnung getragen werden. Die Aufgabe des Jahrbuchs besteht darin, die im Rahmen der Sektionstagungen geführten Diskussionen zu dokumentieren und damit einen Einblick in den aktuellen Stand der Berufsbildungsforschung zu geben. Mit dem hier vorgelegten fünften Jahrbuch sollen die Arbeiten der Sektion Berufs- und Wirtschaftspädagogik erneut einer interessierten Öffentlichkeit zugänglich gemacht werden.

Die im September 2016 an der Universität Hamburg durchgeführte Jahrestagung der Sektion Berufs- und Wirtschaftspädagogik der DGfE erfreute sich einer sehr guten Resonanz. Das aktuelle Jahrbuch präsentiert daher insgesamt elf Beiträge, die wie gewohnt einem doppelten Reviewverfahren unterzogen wurden.

In einem ersten Abschnitt widmet sich das Jahrbuch zunächst ausgewählten Fragestellungen disziplinärer Selbstvergewisserung der Berufs- und Wirtschaftspädagogik. Karin Büchter diskutiert in ihrem grundlegenden Beitrag mit dem Titel „Zum Gehalt berufs- und wirtschaftspädagogischer Selbstthematisierungen - Rückblick und Ausblick" die Frage nach dem disziplinären Selbstverständnis der Berufs- und Wirtschaftspädagogik und zeichnet diesbezüglich die Diskussionslinien seit dem Beginn des 20. Jahrhundert nach. Zentrale Leitlinien bzw. neuralgische Punkte sind Fragen nach der Eigenständigkeit der Disziplin bzw. der Stellung im Gesamtgefüge der Erziehungswissenschaften, der Entwicklung einer Berufsbildungstheorie sowie paradigmatischen Festlegungen.

In dem sich anschließenden Beitrag von Matthias Söll geht es dann um die Orientierung des von der Sektion Berufs- und Wirtschaftspädagogik 2003 erlassenen und 2014 modifizierten Basiscurriculums für die Binnen- und AuBenlegitimität unserer Disziplin. In seinem Beitrag stellt Söll beide Varianten des Basiscurriculums nebeneinander und analysiert diese u. a. hinsichtlich der jeweils verfolgten Ziele, inhaltlichen Schwerpunktsetzungen sowie - vor dem Hintergrund der Vielfalt einschlägiger formeller Vorgaben - deren generelle Bedeutung für die Gestaltung von berufs- und wirtschaftspädagogischen 
Studiengängen. Während das Basiscurriculum von 2003 eher inputorientiert geprägt war, nimmt die Variante von 2014 vornehmlich Zielstrukturen in den Blick. Vor diesem Hintergrund konstatiert Söll: „Einerseits spricht die Berücksichtigung aktueller bildungs- und hochschulpolitischer Rahmenbedingungen im 2. Basiscurriculum dafür, dass dieses bei der Ableitung von Studienzielen, -strukturen und -rahmenbedingungen mehr Orientierung bietet. Andererseits wirkt sich die starke Reduzierung der Präzisierung von Studieninhalten zugunsten der Beschreibung eines kompetenzorientierten Raums möglicherweise negativ auf das Orientierungsvermögen der Leitlinie aus" (S. 38).

Im zweiten Abschnitt des vorliegenden Bandes stehen Beiträge zur beruflichen Lehr-Lern- und Unterrichtsforschung im Mittelpunkt. Christoph Helm, Jacqueline Netzthaler und Bettina Kreuzer analysieren in ihrem Beitrag die Wirkungen des kooperativen Lernens im kaufmännischen Unterricht. Die Analyse zielt auf die Sozialkompetenz und das Sozialverhalten von Lernenden ab. Die Stichprobe umfasst knapp 540 Schülerinnen und Schüler aus 25 Klassen der BHMS (Berufsbildende mittlere und höhere Schulen) in Österreich. Auf Basis von Netzwerkanalysen wird gezeigt, dass leistungsschwächere Lerndende signifikant häufiger von leistungsstärkeren Mitschülerinnen und Mitschüler unterstützt werden als von Leistungsschwächeren. Für Schülerinnen und Schüler, die sich selbst als sozial kompetent einschätzen, können ebenfalls überdurchschnittlich häufig Unterstützungsmaßnahmen registriert werden.

Im Beitrag von Manuela Niethammer und Anke Langner geht es um das Thema Inklusion, das aus einer fachdidaktischen Perspektive sowie mit Blick auf die Modellierung von Lernprozessen beleuchtet wird. Die Autorinnen diskutieren fachdidaktische Gestaltungsmöglichkeiten zur Implementierung inklusionsbezogener beruflicher Lehr-Lern-Settings. Hierzu ziehen die Autorinnen Ansätze der Sachstruktur- und Handlungsstrukturanalyse im Sinne der lehr-lerntheoretischen Didaktik von Heimann, Otto und Schultz (1966) sowie die Entwicklungslogische Didaktik von Feuser (1989) heran, um Potenziale für die „Individualisierung und Differenzierung von Lernwegen“ (S. 65) herauszuarbeiten. In diesem Kontext wird die Subjektorientierung betont. Bezugspunkt der Analysen ist weniger die wissenschaftliche Sicht auf Lerninhalte, sondern viemehr ,die Frage, welches Wissen die sachgerechte Bewältigung der jeweiligen beruflichen Arbeitsaufgaben fundiert" (S. 68).

Svenja Ohlemann und Katja Driesel-Lange berichten über eine Studie zur Individualisierung von berufsorientierenden Angeboten. Dabei steht insbesondere die Kompetenzförderung mittels der Analyse von Lernstilen im Blickpunkt. Zu diesem Zweck werden zunächst das Thüringer Berufswahlkompetenzmodell sowie ein an Kolb angelehntes Lernstilkonzept näher beleuchtet und anschließend beide Diagnostikinstrumente verschränkt. Zur empirischen Prüfung werden über 500 Jugendliche zu ihren Lernstilen und Be- 
rufswahlprozessen befragt. Es zeigt sich, dass bei den Jugendlichen unterschiedliche Lernstile grundsätzlich differenzierbar waren. Die empirische Verknüpfung der beiden Messinstrumente liefert dann weiterführende, angesichts einiger Restriktionen der Studie allerdings mit Bedacht zu interpretierende Hinweise auf die Bedürfnisse von Jugendlichen bei der Berufswahl.

Mandy Hommel, Bärbel Fürstenau, Claudia Leopold, Héctor Ponce und Mario López schließlich untersuchen mittels einer explorativen Studie, „ob Online-Informationen von Banken den Erwerb von Wissen über Baufinanzierung und finanzkompetente Entscheidungen potentieller Darlehensnehmer/ -innen unterstützen“ (S. 97f.). Zu diesem Zweck werden die Webseiten zweier Banken herangezogen, die sich bezüglich des Umfangs und der Qualität der dargebotenen Informationen unterscheiden. Insgesamt 101 Studierende verschiedener wirtschaftswissenschaftlicher Studienrichtungen bearbeiten eine komplexe Aufgabe zur Baufinanzierung (in den Experimentalgruppen mit Hilfe der Bankwebsiten inklusive der dort vorfindbaren Hilfsmittel wie Baudarlehenskalkulatoren). Aus den Befunden lässt sich schließen, dass die von den Banken zur Verfügung gestellten Informationen kaum zur Steigerung der Finanzkompetenz in Bezug auf Entscheidungen über Baufinanzierung beitragen.

Der Schwerpunkt des dritten Abschnitts des Jahrbuches liegt auf Fragen der Hochschul- und Lehrerbildungsforschung. Zunächst berichten Silke Lange und Dietmar Frommberger über eine Studie zur Ausgestaltung schulischer Praxisphasen im beruflichen Lehramtsstudium. Mittels einer Befragung (die Stichprobe umfasst 36 Studienstandorte) werden Strukturen und Modelle der aktuellen Schulpraxisphasenmodelle im beruflichen Lehramtsstudium herausgearbeitet. Von Interesse ist inbesondere, inwieweit diese Konzepte die Spezifika des beruflichen Lehramts(-studiums) widerspiegeln. Die Analysen zeigen, dass die curriculare Einbindung durch Seminare und praktikumsübergreifende Konzepte zwar unterschiedlich ausfällt, aber doch häufig gegeben ist. Insgesamt ergibt sich ein heterogenes Bild - die schulpraktischen Ausbildungsmodelle sind vielfältig; es hat sich ein breites Spektrum etabliert. Die Autorin und der Autor sprechen hier von einem „Flickenteppich“ (S. 124).

Heike Jahncke und Karina Kiepe stellen im Anschluss eine Studie zum Einsatz und der Evaluation eines Tagungsportfolios im Rahmen der Lehrerbildung vor. Vor dem Hintergrund der recht geringen Akzeptanz des Portfolioansatzes im Vorbereitungsdienst setzen die Autorinnen bei den Ausbildungsverantwortlichen - nämlich den Fachleiterinnen und Fachleitern - an und bitten diese, im Rahmen einer Tagung des Bundeskreises der Seminarund Fachleiter/-innen (Schwerpunkt der Tagung war das Themenfeld Reflektieren und Beraten) ein Tagungsportfolio zu führen. Die mehrstufig angelegte Evaluation verweist auf durchaus positive Effekte des Einsatzes des Tagungsportfolios. 
Julia Warwas und Andreas Rausch präsentieren die Ergebnisse einer fächervergleichenden Analyse von unterrichtlichen Überzeugungen und Praktiken von Lehrkräften an Beruflichen Oberschulen. Die Stichprobe umfasst 29 Lehrkräfte sowie 550 Lernende aus den jeweiligen Klassen. Dabei zeigt sich, dass - unabhängig von der Fachkultur und in Übereinstimmung mit verschiedenen Beobachungsstudien - den Sozialformen Lehrvortrag und fragendentwickelndes Lehrgespräch eine große Bedeutung zukommt. Bezüglich der Frage, inwiefern unterrichtsbezogene Überzeugungen und Praktiken fachkulturell geprägt sind, ergeben sich dann weiterführend einige interessante Befunde insbesondere dahingehend, dass Lehrkräfte in wirtschaftswissenschaftlichen sowie mathematisch-naturwissenschaftlichen Fächern transmissiven Lehrstrategien einen höheren Stellenwert beimessen als eher konstruktivitischen Ansätzen.

Christan Schmidt berichtet dann über eine Studie zur Öffnung des Hochschulzugangs für beruflich Gebildete. Im Beitrag geht es darum, die Selbsteinschätzung von studiumsbezogenen Schlüsselkompetenzen mit Portfolioarbeit und binnendifferenzierten Maßnahmen zu verbinden. Aus hochschuldidaktischer Sicht orientiert sich der hier vorgestellte Ansatz am Constructive Alignment und zielt auf eine enge Abstimmung von Lehre, Kompetenzerwartungen und Assessment ab.

Der Beitrag von Marc Casper, Bernadette Dilger, Frederik Fischer, Katharina Fütterer, Nicole Naeve-Sto $\beta$ und Tade Tramm (Abschnitt IV: Schulentwicklungsforschung) beschließt das Jahrbuch. Die Autorinnen und Autoren geben einen Überblick über die Befunde zweier Projekte zur Förderung der Entwicklung von beruflichen Schulen im regionalen Verbund. Es lassen sich postive Effekte regionaler Verbünde ausmachen, die erstens auf Größenund Effizienzeffekte, zweitens auf Innovationseffekte durch gemeinsam entwickelte Profekte sowie drittens auf Qualitätseffekte durch den kollegialen Austausch zurückgeführt werden können.

Der Vorstand der Sektion Berufs- und Wirtschaftspädagogik der DGfE dankt sehr herzlich Herrn Professor Dr. Jens Siemon (Universität Hamburg) sowie den dortigen Mitarbeiterinnen und Mitarbeitern für die Ausrichtung der Sektionstagung. Unser Dank gilt zudem den Gutachterinnen und Gutachtern, die die eingegangenen Beiträge in gewohnt akribischer Manier unter die Lupe nahmen und viele konstruktive Hinweise zur Verbesserung der Texte lieferten. Schließlich danken wir Frau Lena Heinrichs für die Mühe bei der Formatierung der Beiträge.

Mannheim, Göttingen und Darmstadt, im Juli 2017

Jürgen Seifried, Susan Seeber und Birgit Ziegler 


\title{
Zum Gehalt berufs- und wirtschaftspädagogischer Selbstthematisierungen - Rückblick und Ausblick
}

\author{
Karin Büchter
}

\section{Einleitung}

Während in Einführungsschriften, Handbüchern, Festschriften und Aufsätzen kontinuierlich Aussagen zum berufs- und wirtschaftspädagogischen Selbstverständnis getroffen werden, finden Kell $(2014,53)$ zufolge, direkte Diskurse und gemeinsame Bilanzierungen über das Selbstverständnis nicht statt. Das Ausbleiben von solchen Diskursen kann ein Zeichen für einen fehlenden disziplinären Problemdruck sein oder aber für bereits verhärtete Abschottungen von Positionen und Forschungsrichtungen, die für elementare Inhalte und Fragen von Selbsthematisierungen blind gemacht haben. Im folgenden Beitrag geht es um die Frage, welchen Verlauf die berufs- und wirtschaftspädagogische Selbstverständnisfrage genommen hat, welche neuralgischen Punkte und kategorialen Bezüge dabei zur Diskussion standen und stehen sollten.

\section{Aufbruchsstimmungen, Kontroversen und Krisen}

Die Selbstverständnisfrage beginnt am Anfang des 20. Jahrhunderts mit der disziplinären Sichtbarwerdung im Zuge der Errichtung von Lehrstühlen, der Publikation von Grundlagentexten im sich entfaltenden Fachschrifttum und von Einführungsschriften, der Suche nach einer disziplinären Bezeichnung, der Festlegung von Grundkategorien und inhaltlichen Schwerpunkten. Wesentliche Kriterien der Selbstverständnisfrage waren von Anfang an die disziplinäre Eigenständigkeit und Einheitlichkeit. In seiner „Berufsschul- und Wirtschaftspädagogik“ ging es Feld $(1928,54)$ darum, einen ,selbständigen pädagogischen Wissenschaftszweig" aufzubauen. Dieser sollte sich von den anderen ,grundlegend durch Stoffabgrenzung, Methode und Erkenntnisziel“ (ebd.) und durch ,seine besonderen Fragestellungen, die nicht mit den Mitteln bisheriger pädagogischer Einstellung beantwortet werden können“ (ebd.), unterscheiden. Ihm war außerdem daran gelegen, dass sich die „Berufsschul- und Wirtschaftspädagogik“ von einer schulbezogenen Anwendungsdisziplin zu einer wissenschaftlichen Disziplin entwickelt, die im Theoriebereich eine „selbstständige Geltung im Rahmen der Gesamtpädagogik“ 
(V) erlangt. Die damaligen Überlegungen waren an der Kulturphilosophie und Berufsbildungstheorie orientiert, jedoch mit überwiegend axiomatischdeduktiver und dezisionistischer Ausrichtung. Die Verkürzung hermeneutischer Interpretationen von Mensch, Kultur und Gemeinschaft auf programmatische Begriffe wie „Volk“ und „Nation“ begünstigten schließlich den fließenden Übergang der ersten theoretischen Ansätze dieser noch identitätssuchenden Disziplin in die nationalsozialistische Ideologie. ${ }^{1}$ So fand die „endgültige Durchsetzung der Wirtschaftspädagogik als selbständig vertretene Hochschuldisziplin“ (Pleiß 1973, 209 ff.) unter der NS-Diktatur statt. Eine kritische Reflexion ideologischer Funktionalisierbarkeit der Disziplin erfolgte erst viel später und auch nur im kleinen Umfang. ${ }^{2}$ Wie in anderen erziehungswissenschaftlichen Disziplinen wollte man sich nach 1945 durch eine an der Realität orientierte, möglichst objektive wissenschaftliche Ausrichtung der Berufs- und Wirtschaftspädagogik von dem Vorwurf politischideologischer Verführbarkeit befreien. So sprach Schlieper $(1949,42)$ von einer ,bedeutsame[n] Wandlung in der Auffassung der wirtschaftspädagogischen Wissenschaft" und zwar von einer ,primär normativen zu einer vorwiegend empirischen“ (ebd.). Der Wissenschaftscharakter sollte durch eine stärker induktive und empirisch-phänomenologische Vorgehensweise bestimmt sein, die sich dem ,ganzen Menschen entsprechend seiner Bestimmungen in einer [...] bestimmten Lebenssituation" (Schlieper 1954/1967, 72) verpflichtet fühlt, und danach fragt, "welche erzieherischen Probleme“ dadurch entstehen, daß der ,in der Wirtschaft wirkende Mensch [...] einen erheblichen Teil seines Lebens in der Wirtschaft zubringt" (Abraham 1953, 22). Obwohl im Laufe der 1950er Jahre unterschiedliche empirische Untersuchungen (vgl. Dörschel 1960, 23) durchgeführt wurden, entfachte sich am Ende dieses Jahrzehnts eine ,wissenschaftliche Kritik an der Wirtschaftspädagogik“ (Linke 1961, 737), und zwar ,sowohl im internen Kreis der Fachvertreter als auch in der fachfremden Öffentlichkeit“ (ebd.), die „mit zum Teil erheblichem emotionalen Engagement" (Kiehn 1962/67, 36) geführt wurde. Fragen nach dem wissenschaftlichen und disziplinpolitischen Selbstverständnis beschäftigten aber die Erziehungswissenschaft insgesamt. Und ähnlich wie hier bildeten sich auch in der Berufs- und Wirtschaftspädagogik bis in die 1970er Jahre hinein unterschiedliche wissenschaftstheoretische und methodologische Positionen heraus (vgl. Dörschel 1960; Abel 1963; Zabeck 1972; Lipsmeier 1972; Lange 1972), über deren Grundannahmen und praktischen Implikationen immer auch im Zusammenhang mit der Selbstverständnisfrage gestritten wurde. Diese Kontroversen ließen aber keinen Zweifel an der Existenzberechtigung der Disziplin, da sich die Berufs- und Wirt-

1 „Ohne Zweifel vollzog sich damit eine Abwendung von der kulturphilosophisch-humanistischen Berufsbildungstheorie; an die Stelle der kulturpädagogischen trat eine politisch fundierte Erziehungstheorie“" (Pleiß 1971b, 206).

2 Siehe Neumann 1969; Pleiß 1973; Seubert 1977; Kipp \& Miller-Kipp 1994. 
schaftspädagogik wissenschaftsgeschichtlich bis dahin behauptet hatte (vgl. Pleiß 1973). Trotzdem gelang es in den Folgejahren nicht, den Diskurs der 1960er/1970er Jahre zu kultivieren und zu etablieren, stattdessen provozierte die weitere disziplinäre Binnendifferenzierung eine erneute Krisenstimmung, die man, so Stratmann (1979/1999, 509), durchaus als „Unsicherheit im Selbstverständnis der Berufs- und Wirtschaftspädagogik“ deuten könnte. Die verschiedenen Forschungsrichtungen könnten aber auch Ausdruck wissenschaftlichen „Reichtums“, der „Offenheit“ und „Lebendigkeit dieser Disziplin“ (ebd.) sein. Darum seien die erkannten „Identitätsprobleme alles andere als ein Krisensymptom" (ebd.). Aber ähnlich wie in der Erziehungswissenschaft wurde auch in Folgejahren die innere disziplinäre Vielfalt nicht als konstruktiv zu bearbeitendes Phänomen, sondern eher als „Systemproblem“ (Tenorth 2004, 382) begriffen. Ohne im Einzelnen wissenschaftshistorisch und -theoretisch zu erläutern, was disziplinäre Einheit ausmacht und welches ihre Vorteile sind, kritisierte die Deutsche Forschungsgemeinschaft (1990, $18 \mathrm{f}$.), dass ,die Berufs- und Wirtschaftspädagogik [...] bislang noch nicht zu einem einheitlichen Wissenschaftsverständnis gefunden [hat]". Da eine ausführliche Verständigung über Einheit und Idendität zu dieser Zeit fehlte, mussten disziplinexterne arbeitsmarkt- und berufsbildungspolitische Entwicklungen der 1990er Jahre, die als „Krise des Berufs“ und „Krise der Lehrerbildung" thematisiert wurden, zwangsläufig in die Frage münden: „Wird die Berufs- und Wirtschaftspädagogik auch zukünftig benötigt werden?" (Schmiel \& Sommer 2001, 18). Parallel zu solchen disziplinären Unsicherheiten, die seit dem Ende der wissenschaftstheoretischen und methodologischen Kontroversen der 1970er Jahre eher deprimiert und hilflos vorgetragen wurden, hat sich die Disziplin intern immer weiterentwickelt. Obwohl genaue szientometrische Analysen über die strukturelle und inhaltliche Differenzierung der Berufs- und Wirtschaftspädagogik noch ausstehen, ist deutlich, dass sich theoretische Positionen und Bezüge, Forschungsinteressen, methoden und -instrumente ausgeweitet haben. Gegenstände werden nicht mehr nur aus der Praxis gewonnen, sondern auch konstruiert, und zwischen Alltagserfahrungen in Arbeit, Beruf und Berufsbildung und wissenschaftlichem berufs- und wirtschaftspädagogischen Wissen sind immer mehr Betrachtungsebenen entstanden. Ein Diskurs quer zu den Paradigmen blieb aber aus, eher verstärkte sich eine Abschottung von Theorien und Forschungsschwerpunkten, wodurch auch das Interesse an Auseinandersetzungen mit der „Krisenanfälligkeit“ (Lisop 2009), den „,chronischen Krankheiten“ (Lempert 2009) oder der Tatsache, dass die Berufs- und Wirtschaftspädagogik „immer noch keinen disziplinären Ort gefunden“ (Bank 2009) habe, gering blieb. 


\section{Neuralgische Punkte in der Selbstverständnisfrage}

Um das Selbstverständnis diskutierbar zu machen, werden im Folgenden auf der Basis bisheriger Selbstthematisierungen neuralgische Punkt der Disziplin rückblickend und ausblickend betrachtet.

\subsection{Appendix der Erziehungswissenschaft?}

Das Verhältnis zwischen Berufs- und Wirtschaftspädagogik und Erziehungswissenschaft war nie eindeutig. Schon in ihrer Konstitutionsphase stand die damalige Berufs- und Wirtschaftspädagogik mit ihrer auf Beruf und Wirtschaft bezogenen Vorstellung von Pädagogik gegen die neuhumanistische Idee der zweckfreien Bildung. Die ersten Lehrstühle wurden auch nicht an philosophischen oder pädagogischen Fakultäten errichtet (vgl. Stratmann \& Bartel 1975, XV). Die Berufs- und Wirtschaftspädagogik kam erst über den Weg der Fachwissenschaften und der fachdidaktischen Notwendigkeit einer Ausbildung von Berufsschullehrern zur Pädagogik. Die Wirtschaftspädagogik hat sich ab 1923 mit eigenen Lehrstühlen zunächst als Handelsschulpädagogik aus den Handelswissenschaften der Handelshochschulen entwickelt. Um 1930 wurde sie zu einer eigenständigen Disziplin und schöpfte ihren Erkenntnisgegenstand nicht ausschließlich und vorrangig aus der erziehungswissenschaftlichen Theorie, sondern auch aus der Wirtschafts- und Sozialpolitik (vgl. Schürholz 1928), der Psychologie (vgl. Kautz 1929) und der Betriebswirtschaftslehre (vgl. Krasensky 1935). Die Gewerbelehrerbildung fand seit Ende des 19. Jahrhunderts zunächst in Gewerbelehrer-Seminaren, später an Berufspädagogischen Instituten, seit den 1920er Jahren auch an Technischen Hochschulen (Karlsruhe 1922, Dresden 1923, Stuttgart 1924, Braunschweig 1925) und Universitäten (Jena 1924, Hamburg 1930) statt, „wobei an den Universitäten die Pädagogik für Gewerbelehrer stärkeres Gewicht erhielt als an den Technischen Hochschulen" (Baumgardt 1976, 11). Nach Abel wurde das Grundwort Pädagogik dem Bestimmungswort Beruf erstmals 1921 im Rahmen einer „Berufspädagogischen Woche“ in Berlin zugeordnet (vgl. Abel 1963, 9). Die Übernahme der Begriffe Pädagogik und zunehmend auch Erziehungswissenschaft durch die gesamte Lehrerbildungsbewegung zu Beginn des 20. Jahrhunderts veranlasste auch die damalige Berufs- und Wirtschaftspädagogik dazu, sich stärker erziehungswissenschaftlich zu orientieren. Hierbei ist zu berücksichtigen, dass in dieser Zeit auch die Erziehungswissenschaft um ihre Autonomie rang und im Wissenschaftssystem selber noch umstritten war (vgl. Tenorth 2004, 359 f.). Dennoch war ihr Einfluss auf die berufs- und wirtschaftspädagogischen Denkformen deutlich. Für Feld (1928) stand fest, dass ,die Berufsschul- und Wirtschaftspädagogik“ eine „auf kulturphilosophischer Basis ruhende erziehungswissenschaftliche 
Disziplin“ (S. 53) ist. Ein Grundsatzstreit darüber, ob die Berufs- und Wirtschaftspädagogik überhaupt als Erziehungswissenschaft möglich sei, entfachte sich in den 1960er Jahren. Hatte Schlieper $(1952,423)$ die Wirtschaftspädagogik noch als „Wirtschafts- und Sozialwissenschaft mit erziehungswissenschaftlicher Schau“ bezeichnet, wies Linke $(1961,737)$ auf die Tendenz der Wirtschaftspädagogik ,zu einem mechanistischen und instrumentalistischen Erziehungsdenken“ hin und warnte davor, dass sie sich ,in ihren Formulierungen und Vorstellungen vom bisher üblichen Erziehungsdenken entfernt“ (ebd.). Deshalb sah er es als Aufgabe an, ,die Absonderung der Wirtschaftspädagogik von der Pädagogik überwinden zu helfen“ (ebd., 738). Die Befürchtungen gingen also dahin, die Disziplin könnte den Anschluss an die Erziehungswissenschaft verlieren. „Es ist kaum zuviel gesagt, daß die Wirtschaftspädagogik auf dem Wege zum Selbstverständnis [...] ziemlich weit hinter den Klärungen der allgemeinen Pädagogik hinterherhinkt" (Schwarzlose 1961, 766). Zu dieser Zeit hatte sich die Berufs- und Wirtschaftspädagogik noch nicht als Doppeldisziplin konsolidiert, so dass es auch um die Frage ging, ob eher die Wirtschafts- oder die Berufspädagogik als Pädagogik zu bezeichnen sei. Groothoff (1961) wies darauf hin, dass sich die Wirtschaftspädagogik ,anders als alle anderen besonderen Pädagogiken, anders auch [...] als die ,Berufs'-Pädagogik [...] als Sezession konstituiert" hat (S. 780). Deutlich würde, dass sich auch ,die Allgemeine Pädagogik, und zwar seit langem, fast ausschließlich mit der Berufspädagogik [...] auseinander[setzt]“" (S. 774). Der Wirtschaftspädagogik fehle die Historisierung der wirtschaftlichen, gesellschaftlichen und technologischen Phänomene, eine „Situationsanalyse“ in Form von Gesellschafts- und Kulturkritik sowie eine „humane Bestimmung der Wirtschaft" (S. 781). Auch Ritzel $(1961,753)$ war davon überzeugt, „daß es nur eine legitime Wirtschaftspädagogik gibt, eben die Berufspädagogik“: „Wir bestreiten den Erkenntnisanspruch und Wissenschaftscharakter der Wirtschaftspädagogik zugunsten dessen der Berufspädagogik [...]. Sie [die Berufspädagogik] stellt die Fragen, zu denen sich die Frage der einen und ganzen Pädagogik im Hinblick darauf differenziert, daß der, Weg zu der höheren Allgemeinbildung über den Beruf und nur über den Beruf" führt" (ebd.). Der zweite umfassendere Selbstverständnisdiskurs problematisierte erneut das Verhältnis zwischen Erziehungswissenschaft und Berufs- und Wirtschaftspädagogik. Während Lange (1971/1975, 308) feststellte, dass „kein Zweifel darüber bestehen [kann], daß der Forschungsstand [...] noch größer ist als sonst in der deutschen Erziehungswissenschaft", kritisierte Lipsmeier $(1972,22)$, dass ,die Rezeption des wissenschaftstheoretischen und erziehungswissenschaftlichen Standards [...] so gut wie ausgeblieben [sei]“. Zabeck (1972) resümierte, die Berufs- und Wirtschaftspädagogik habe „keine eigene Methode, keine eigene Terminologie, keinen eigenen Theoriebegriff" (S. 586) und müsse daher auf die erziehungswissenschaftliche Grundlagendiskussion zurückgreifen (S. 585), jedoch dürfe nicht 
verkannt werden, dass es die Erziehungswissenschaft selber nicht vermocht hat, „einen Konsens über ihren Wissenschaftscharakter herbeizuführen“ (S. 577) und „,von einer Konsolidierung ihres Selbstverständnisses [...] weit entfernt" (ebd.) ist. Am Ende dieser Auseinandersetzung stand fest, dass die Berufs- und Wirtschaftspädagogik nicht in der Lage ist, von sich aus und innerhalb ihres eigenen Radius Gesellschafts- und Bildungsfragen zu beantworten. Ohne erziehungswissenschaftliche Anbindung laufe sie Gefahr, zur didaktischen Variante der Fachwissenschaften zu degradieren und die angehende Lehrerschaft ohne gesellschaftskritische Reflexion auf ihre Tätigkeit vorzubereiten (vgl. Stratmann \& Bartel 1975, XIII f.). Inwieweit es der damaligen Berufs- und Wirtschaftspädagogik tatsächlich gelungen ist, deutlich zu machen, dass die Erziehungswissenschaft auch auf sie angewiesen ist, ist bis heute nicht klar. Doch nach wie vor klammert die Erziehungswissenschaft die berufliche Integration der Jugend in die Arbeitswelt, die pädagogische Bedeutung von Beruf, Arbeit, Wirtschaft und Technik und die Funktionsweisen von Berufsbildung aus ihren Reflexionen und Untersuchungen weitgehend aus (vgl. ebd.).

Mit dem Vorsatz einer intensiven Verständigung über erziehungswissenschaftliche und berufs- und wirtschaftspädagogische Ziele, Vorgehensweisen und Praktiken wurde die Berufs- und Wirtschaftspädagogik auf der DGfETagung am 10. April 1972 in Nürnberg der Erziehungswissenschaft formal zugeordnet. Auch wenn es immer mal wieder Überlegungen dazu gab, wie das Verhältnis zwischen Erziehungswissenschaft und Berufs- und Wirtschaftspädagogik gestaltet werden kann, sind bis heute das Verhältnis und seine historischen Hypotheken, die Anlässe und Schwerpunkte diskursiver Wechselbeziehungen, gemeinsame Grundverständnisse, ebenso wie gegenseitige Zuschreibungen und Abgrenzungen noch nicht ausführlich aufgearbeitet worden.

\subsection{Objektivitätsanspruch und Normativitätsdilemma}

Ein historisch kontinuierliches Bemühen der Berufs- und Wirtschaftspädagogik besteht darin, in ihrer wissenschaftlichen Ausrichtung nicht als zu normativ und anwendungsorientiert dazustehen, sondern paradigmatisch offen $\mathrm{zu}$ sein. So heißt es bei Feld $(1928,54)$ : „Was den wissenschaftlichen Aufbau betrifft, können wir hier [...] drei Wege gehen, spekulativ, empirisch und beides vereint. Für unsere beruflich eingestellte Pädagogik haben wir uns ohne weiteres für das letzte entschieden". Gedacht war also an eine Verbindung von empirischen Erkenntnissen mit pädagogischen Werten. Eine solche Kombination hatte auch Dörschel $(1960,30)$ vor Augen. Aufgrund ihres „empirisch-deskriptiv-explikativen“ Verfahrens sei „die Wirtschaftspädagogik [...] auf dem Weg zu einer eigenständigen Tatsachenforschung, ohne die 
normative, die begriffsorientierte erziehungsphilosophische, die vergleichende und historische Forschung zu vernachlässigen". Da sich das Normative aber nicht mit dem Anspruch reiner Wissenschaftlichkeit vertrug, die Berufsund Wirtschaftspädagogik sich andererseits auch als pädagogischpraxisbezogene Disziplin verstehen musste, stellte sich die Verknüpfung als nicht widerspruchsfrei dar. Für Zabeck $(1968,92)$ sollte sich die Wissenschaftlichkeit ,allein in der Hingabe, mit der der einzelne Fachvertreter der Idee der Wahrheit dient“, „,im Streben nach ,intellektueller Redlichkeit“ und ,Wahrhaftigkeit““, im Widerstreben „der ,pateiischen Argumentation““ und in der Mitteilung der Erkenntnisse ,in ,rationaler Zucht und begrifflicher Sauberkeit'“ äußern. Eine Normgebundenheit holte ihn aber dann mit seiner axiomatisierten Berufs- und Wirtschaftspädagogik wieder ein (ebd., 594 ff.). Die gesellschaftskritische und emanzipatorische Berufspädagogik richtete das Erkenntnisinteresse ihrer Forschung gezielt auf Herrschaftskritik, die Förderung von Selbstbestimmung und die Beseitigung von Minderprivilegierung (vgl. Lempert 1972). Vor dem Hintergrund des Werturteilstreits in den Sozialwissenschaften kam es schließlich auch in der Berufs- und Wirtschaftspädagogik zu unversöhnlichen Auffassungen von Wissenschaftlichkeit und Erkenntnisinteressen. Seit den 1990er Jahren war es dann die Modellversuchsforschung, die Kontroversen um das berufs- und wirtschaftspädagogische Methodologieverständnis zwischen angewandter und Grundlagenforschung provoziert hat (vgl. Beck 2003). Mit der überwiegend quantitativ ausgerichteten „empirischen Bildungsforschung“ seit Beginn der 2000er Jahre gab es für die Berufs- und Wirtschaftspädagogik einen Anlass, sich dem Verdacht einer überwiegend anwendungsorientieren Disziplin zu entledigen. Neben der bereits gut situierten empirischen Lehr- Lernforschung konnte nun die empirische Kompetenzforschung eine weitere Alternative zur Anwendungsforschung sein, da es hier um objektive Empirie gehen sollte. Doch was der Modellversuchsforschung an Theorie-, Empiriedefiziten und vereinfachter Modelltechnokratie vorgeworfen wurde, gilt ähnlich für die Kompetenzforschung. Wie in der empirischen Bildungsforschung war auch in der Berufs- und Wirtschaftspädagogik mit der Kompetenzorientierung von vornherein eine ,symbiotische[n] Beziehung des Kompetenzbegriffs zu diagnostischen Messverfahren“ (Dammer 2015, 125) gegeben. Die für theoretische Grundlegung herangezogenen erziehungswissenschaftlichen Kompetenztheorien wie die von Heinrich Roth werden pragmatisch so verkürzt, dass sie den methodischen Messmöglichkeiten und Messzielen angepasst werden können. Bildungsphilosophische und -theoretische Einwände gegen gesellschaftsunkritische und subjekttheoriearme Kompetenzverständnisse, gegen die weitgehende Reduktion von Kompetenzen auf Können, gegen die sozialschichtspezifische Gebundenheit von Kompetenzanforderungen und gegen die Auffassung von Lernsubjekten „als kausal funktionierende Apparaturen“ (ebd.) haben bislang kaum Eingang in die berufs- und wirtschaftspädagogische 
Kompetenzforschung gefunden; ${ }^{3}$ auch nicht die Frage, ob und inwieweit eine reflexive pädagogische Professionalisierung herkömmlicher und schulalltäglichen Kompetenzdiagnostik im Rahmen von Lehrertätigkeit nicht sinnvoller mit der Lehrerbildung verknüpft werden könnte als schulexterne Kompetenzmessungen, deren praktische und didaktische Wirksamkeit ohnehin fraglich ist. Schließlich steht auch die empirische Kompetenzforschung, die sich als wertfrei verstehen möchte, vor Objektivitätsgrenzen und einem Normativitätsdilemma, da sie die Bezugsgrößen ihrer Messung (Anforderungen der Arbeitswelt, curriculare Vorgaben) unhinterfragt setzt. Übersehen wird dabei das, was Heid $(1977,836)$ zu Bedenken gab, „daß es zwischen den genannten Gliedern des ,Ableitungszusammenhangs' logisch, theoretisch und empirisch begründ- und überprüfbare Vermittlungsprozesse und -entscheidungen höchster Komplexität gibt“, die zu den Fragen führen: „Welches Merkmal [...] wird warum als ,Anforderung' selegiert und definiert; warum bleiben andere Merkmale außer Betracht? Welche Anforderung wird warum als erhebungs- bzw. erziehungsrelevant ausgewählt? ([...] Welches Verhalten wird warum jeweils als anforderungsbedeutsam postuliert und welches Verhalten dann tatsächlich jeweils als Erfüllung (oder Verfehlung) der Anforderung honoriert (oder sanktioniert)?" (ebd.). Die empirische Kompetenzforschung ist hier begründungspflichtig, wenn sie sich nicht den Vorwurf gefallen lassen will, ihr Erhebungsinstrumentarium aus fraglosen Plausibilitäten abgeleitet zu haben, einem Mangel an kritischer Distanz zur eigenen Praxis und einer Verflochtenheit der Forschung mit politischen Forschungsfördertrends und deren Sachgesetzlichkeiten, die eine Ökonomie des Sehens erforderlich machen, zu unterliegen.

\section{3 „Politische Askese“, politische Steuerung und Diskursregime}

Trotz des Vorsatzes „,politischer Askese“ (Zabeck 1972, 581) steht Wissenschaft immer in einem gesellschaftlich-politischen Kontext (vgl. Albert 1991, 36). Sie setzt einen Sinnzusammenhang voraus, der gesellschaftlich bestimmt ist und damit in Entstehung und Vollzug abhängig von Politik. Gleichzeitig liefert sie der Politik mit ihren wissenschaftlichen Ergebnissen Argumente und Legitimationen. So gerät auch , die Berufs- und Wirtschaftspädagogik zur Sicherung ihrer Außenlegitimität und damit ihrer Ressourcenbasis zunehmend in den Sog von Politik und Wirtschaft" (Reinisch 2009, 13). Ähnlich Lisop $(2009,6)$ : „Betrachten wir die Berufs- und Wirtschaftspädagogik im Spiegel historischer Etappen, dann sehen wir deutlich, dass ihre Themen, ihre jeweilige institutionelle Stabilität, Stärke oder Schwäche und auch die wissenschaftliche Selbstreflexion bzw. Identität stark an die allgemeine Politikentwicklung angelehnt waren, speziell an deren ökonomische Basis“.

3 Vgl. neuerdings die „Antworten auf die Kritiker“ (Baumert/Tillmann 2017). 
Trotzdem ist die Berufs- und Wirtschaftspädagogik keine politisch reaktive Disziplin. Die Beziehung zwischen systematischem Wissen und politischem Handeln ist nicht einseitig, vielmehr handelt es sich „um ein Bedingungsverhältnis zwischen unterschiedlichen Konfigurationen politischer Zielformulierung und wissenschaftlicher Entwicklung (Weingart 1976, 167). So werden beispielsweise Forschungsförder- und Berufsbildungspolitik von Ausschüssen, Gremien, informellen Beratungen gesteuert, an denen die Berufs- und Wirtschaftspädagogik beteiligt ist. Deshalb ist weniger die Frage relevant, ob sie an Politik partizipiert, sondern vielmehr, in welcher Weise sie mit ihr verflochten ist, welchen Einfluss sie hat und wer festlegt, was als vernünftiges und richtiges Handeln gelten kann, inwieweit sie jenseits übergreifender Orientierungen von sich aus Probleme auf die politische Agenda setzt, Meinungen mitproduziert, in welchem Verhältnis wissenschaftliche und politische Probleme zueinander stehen, und wie die Übersetzungen zwischen beiden im konkreten Beratungsprozess ablaufen (vgl. Weingart 2003, 92). Für die Berufs- und Wirtschaftspädagogik ist die Verflochtenheit mit Politik, das Einsickern politischer Strömungen in die innere Wissenschaftsstruktur und ihre Einflussnahme auf politische Steuerungen und Entscheidungen schwer rekonstruierbar. Nicht unwesentlich ist in diesem Zusammenhang auch die Frage, wie und wodurch die Wissensproduktion im disziplinären Binnenverhältnis gelenkt wird, inwiefern und welche disziplinären Diskursregime durch Forschungs- und Berufsbildungspolitik zustande kommen, die theoretische und methodische Reflexionen oder Nicht-Reflexionen steuern, Probleme, Programme, Instrumente der Forschung als richtig und wichtig definieren, festlegen, was Plausibilität beanspruchen kann und was nicht, was oder wessen Positionen als wahr und legitim gelten und welche nicht, was der „Jargon der Eigentlichkeit" ist, welche Denkstile in die Hochschullehre hineingetragen werden und die inhaltliche Ausrichtung von Professuren bestimmen. Fraglich ist auch, ob und inwieweit disziplininterne Abschottungen von Forschungssträngen der Logik von forschungsförder- und berufsbildungspolitischen Abhängigkeiten folgen. Die Verstricktheit der Berufs- und Wirtschaftspädagogik mit Politik muss aber nicht zwangsläufig auf Kosten der Binnenlegitimität gehen, nämlich dann nicht, wenn die politische Involviertheit der disziplinären und wissenschaftlichen Kritik zugänglich wird (vgl. Reinisch 2009, 13).

\section{Selbstverständnis über Grundbegriffe}

Die Verständigung über disziplinäre Grundbegriffe, deren Gehalt und Widersprüche, bildet eine Grundlage für Selbstverständnisdiskurse. Zu den Grundkategorien der Berufs- und Wirtschaftspädagogik gehören zunächst die im 
Disziplinnamen enthaltenen Begriffe: Beruf, Wirtschaft, Pädagogik bzw. Bildung.

\subsection{Beruf}

Beruf ist eine Zentralkategorie der Berufs- und Wirtschaftspädagogik. Kritische Auseinandersetzungen mit dem Beruf tangieren seit der zweiten Hälfte des 20. Jahrhunderts auch die Selbstverständnisfrage. Da der Beruf seit der Industrialisierung in Frage steht (vgl. Abel 1963), ist ein berufs- und wirtschaftspädagogischer identitätsbildender Kern seit Disziplingründung fragil. Dass die Berufsbildungstheorie und die durch sie beeinflusste Berufs- und Wirtschaftspädagogik vor der heterogenen und widersprüchlichen Berufswirklichkeit lange Zeit die Augen verschlossen und sich dabei ,auf merkwürdige Mystifizierungen, auf Unterscheidungen von echtem und unechtem Beruf" (Stratmann 1975, 307) eingelassen haben, wurde ihr in den 1970er Jahren ,als eine der schwersten Hypotheken“ (ebd., 306) vorgeworfen. Noch zwanzig Jahre später schreibt Kutscha (1992, 538): „Die Berufs- und Wirtschaftspädagogik täte mithin gut daran, sich mit den Widersprüchen und Paradoxien auseinanderzusetzen, die aus der Gleichzeitigkeit disparater Entwicklungen bzw. Entwicklungszustände resultieren“. Grund für die Berufsproblematik ist die Diskrepanz zwischen einem abstrakten Beruf und den konkreten Inhalten von Arbeit. Berufe sind nicht logische Folgerungen von Arbeitsprozessen, sondern Ergebnisse sozialer Aushandlungen, in die mit Bildungsansprüchen interveniert werden könnte. Eine politikorientierte Perspektive haben in den 1980er Jahren die Vertreter der gestaltungsorientierten Berufsbildungsforschung (vgl. Rauner 1985) eingenommen, um im Sinne von Sozialverträglichkeit und Mitbestimmung Planungsprozesse von Berufen zu hinterfragen, die für Berufe charakteristischen Aufgaben zu identifizieren und $\mathrm{zu}$ modellieren. Anstatt diese Perspektive berufsfeldübergreifend $\mathrm{zu}$ diskutieren, verblieb sie im engen Kreis berufswissenschaftlicher Forschung und ihrer gewerblich-technischen Fachdidaktiken. Ansonsten dominierte in der Disziplin ein sachlogisch begründetes evolutionäres Berufsdenken, das sich in verschiedenen Diskurslinien mit unterschiedlichen Szenarien niederschlug. So wurden eine Erosion, eine Dysfunktionalität und eine bedingte Stabilität des Berufs prognostiziert, auch verbunden mit Vorschlägen sich auf andere Kategorien wie Arbeit und Erwerb zu konzentrieren oder über institutionelle und individuelle Flexibilisierungsformen nachzudenken. Neben unkritischen Pauschalrezeptionen von Megatrends sind für die meisten Auseinandersetzungen mit Beruf auch ,pauschale Aussagen über die Berufe, die Beruflichkeit, die Entberuflichung, das Berufskonzept" (Kell 2016, 1) kennzeichnend, so dass kaum Anknüpfungspunkte für erziehungswissenschaftliche, curriculare und didaktische Überlegungen offengelegt werden können. 
Selbstbeschränkend in der Diskussion um Beruf war auch lange Zeit die Fixierung auf das Duale System der Berufsausbildung. Berufsrelevante Entwicklungen jenseits dieser Sphäre, also im Weiterbildungssektor, im Übergangsbereich oder im Hochschulsystem, sind daher schnell als Bedrohung des als eigentlich wahrgenommenen Berufs interpretiert worden. Möglicherweise hätten durch eine weniger standesfixierte Berufsbezogenheit viel früher „Beruf und Wissenschaft als organisierende Prinzipien“ (Reetz 1976) mit Blick auf Akademisierung der Berufsbildung ausgelegt werden können. Vielleicht wäre dann auch die Aufgeschlossenheit für Ideen, wie die der ,erweiterten Beruflichkeit" (Kutscha 2015) mit der Perspektive integrierter Berufsund Bildungskonzepts, größer.

\section{2 Ökonomie}

Die Berufs- und Wirtschaftspädagogik hat es in mehrfacher Hinsicht mit dem Ökonomischen zu tun, als äußere Bedingung, wenn es um die Frage effizienter berufsbildungspolitischer und -institutioneller Steuerung geht, und als Gegenstand, wenn curriculare und fachdidaktische Frage des Wirtschaftsunterrichts verhandelt werden (vgl. Bellmann 2001, 228ff). Das, was beide Ebenen miteinander verbindet, sind grundsätzliche Auffassungen von Ökonomie, über die sich Berufs- und Wirtschaftspädagogik zu verständigen hat, wenn sie Lehrerinnen und Lehrer dazu befähigen will, aus einer „ethisch legitimierten und selbstreflexiv kontrollierten Perspektive Urteile zu bilden“" (Sektion Berufs- und Wirtschaftspädagogik 2014, 5). In der Berufs- und Wirtschaftspädagogik hat sich im Verlauf ihrer Geschichte das Verständnis von Wirtschaft mit den jeweiligen Denkstilen und Theoriebezügen verändert. So verstand Feld $(1928,15)$ Wirtschaft im Kontext seiner Kultur- und Wertphilosophie weniger als „Sphäre des Egoismus“ und „Welt der Ausbeutung“, sondern als „Kultursphäre“. Für Ausbeutung, Missstände in der Bildung und für die Lehrlingszustände dürfe ,nicht die Wirtschaft an sich“ (ebd., 16) verantwortlich gemacht werden, ,sondern die Menschen, die sich ihrer Verantwortung der Gemeinschaft gegenüber entziehen“ (ebd.). Für ihn stand fest, „daß die Wirtschaft ihre eigenen Gesetze hat und betriebswirtschaftliches Denken und Forschen an sich mit der Ethik nichts zu tun hat" (ebd., 18). Dennoch müsse „Wirtschaftlichkeit als Norm unseres werktätigen Lebens und Handels" (ebd., 21) verstanden werden, damit der Mensch dem Wirtschaftsprozess nicht feindlich gegenübersteht, sondern ihn begreift und als „Teilfunktion“ in Gesellschaft und Betrieb hinnimmt. Das Ziel der „wirtschaftlichen Bildung" liege im Verständlich machen der gegebenen wirtschaftlichen Vorgänge (vgl. ebd., 27). Schlieper $(1963,110)$ grenzte sich von der Auffassung ab, dass ,das ,wirtschaftliche“ Handeln eigene unabhängige Normen und Gesetze beanspruche“ und stellte heraus, dass die „Wirtschafts- 
form“ (ebd.) der Wirtschaftsauffassung, „die sie beherrscht“ (ebd.), entspricht. Verschiedene Wirtschaftsauffassungen wurden von ihm nicht weiter reflektiert, vielmehr folgert er, dass ,,je nachdem, zu welcher erziehungswissenschaftlichen Richtung sich die einzelnen Pädagogen bekennen, [...] sie die Bedeutung des wirtschaftlichen Lebens- und Kulturbereiches für die Erziehung anders einschätzen [werden]“ (ebd., 113). Abraham $(1967,259)$ wies auf die Tendenz der Funktionalisierung des Menschen durch die Wirtschaft hin und insistierte darauf, dass es nicht nur um ,ökonomische Brauchbarkeit“ gehen dürfe, sondern, ,die Kernfrage lautet vielmehr, wie der [moderne Mensch] unter den Existenzbedingungen, die [...] von der Wirtschaft aufgezwungen werden, sein Leben so gestalten kann, daß es nicht nur ökonomisch erfolgreich ist, sondern ihn auch geistig befriedigt". Mit seinen Überlegungen zu „Wirtschaft im Bildungsraum“ wird auch bei ihm ein Verständnis von Wirtschaft als übergreifende Gegebenheit deutlich: „Es ist für die Wirtschaft lebenswichtig, daß sich das Erziehungswesen in Ordnung befindet [und] daß der moderne Mensch innerlich so stark [ist], daß er die innere Freiheit behält, ohne die das gesamte Dasein wertlos ist“" (ebd., 271).

Eine politische Perspektive auf Ökonomie findet sich hingegen bereits bei Fischer (1932/1963, 35): „Ökonomische Kategorien beherrschen heute alles und alle. Man muß es aus dieser uneingeschränkten Vorherrschaft des Ökonomismus in Denkhaltung und Lebenseistellung verstehen, wenn auch der Mensch und seine Bildung dem Geltungs- und Machtbereich der Wirtschaft unterworfen werden. Der Mensch verliert dabei seine Würde, wird Ware". Er thematisierte die durch wirtschaftliche Macht hervorgerufene Ungleichheit, den „Gegensatz zwischen Luxus auf der einen und halszuschnürender Not auf der anderen Seite, die Extreme der Massenverelendung und des sinnlosen Luxus, die Härte eines Rechtes, das solche Extreme gewiß ursprünglich nicht gewollt hat, aber tatsächlich schützt, die Entfremdung der Menschen“ (S. 53). Aus seinen Überlegungen zog er die Konsequenz, die Bildungskräfte des Wirtschaftslebens im „Geist des sozialen Humanismus“ (S. 58) und gerechter Arbeits- und Wirtschaftsordnung (S. 56) zu identifizieren und deren Entfaltung zur Aufgabe aller Schulformen zu machen. Ähnlich kritische Positionen finden sich später bei Linke (1957/1967). Die „Prosperität der Wirtschaft“ sei nicht Maß aller Dinge. Diese dürfe ,immer nur den Charakter eines Zweckmittels“ (ebd.) besitzen. Das Augenmerk „wirtschaftspädagogischer Forschung“ habe sich ,,auf die Gestaltbarkeit der Wirtschaft" (S. 129) zu richten. Er kritisierte auch den ,Zirkel der engen Verflechtung zwischen wirtschaftlicher Erfolgsrechnung und berufspädagogischer Bildungsplanung“" (Linke 1965, 326). Auch Dörschel $(1960,103)$ wollte das „ökonomische Bildungsideal“ aus „,der Idee und dem Phänomen der Menschlichkeit“ und der „Humanisierung der Arbeit“ und als „Antithese zu bestehenden politisch-ideologischen Glaubens- und Denkmustern“ begreifen. Mit gesellschafts- und bildungskritischem Anspruch verfasste Kutscha 
(1976) seinen politisch-ökonomischen Strukturgitteransatz, um durch politisch-ökonomische Bildung im Medium des Berufs eine Grundlage zu bieten, um Bedingungen und Folgen wirtschaftlicher Entscheidungen und Handlungen im Kontext politischer und ethischer Problemstellungen differenziert und kritisch beurteilen zu können. In der Berufs- und Wirtschaftspädagogik gibt es also schon seit längerem bildungskritische Überlegungen, die das „Ökonomische“ nicht mehr vorrangig als ,(materiales) medium quod“ (Bellmann 2001, 236), sondern als ,(formales) medium quo der Bildung“ (ebd.) verstehen. Die Frage ist, welchen disziplinären Stellenwert diese Überlegungen noch oder wieder haben. Oder führen aktuelle Diskursregime zurück zur ökonomischen Sachlogik? Die Überwindung des schon von Fischer (1932/1963) formulierten „Ökonomismus“ gehört heute angesichts der aktuellen Debatten um Ökonomisierung als soziale Regulierungsform zu wichtigen Themen des berufs- und wirtschaftspädagogisches Diskurses, in dessen Zusammenhang auch anders über „ökonomische Kompetenz" diskutiert werden und eine kritische Reflexion von Individualmoral in der Marktwirtschaft und Wirtschaftsethik erfolgen müsste.

\subsection{Bildung}

Im Basiscurriculum der Sektion für Berufs- und Wirtschaftspädagogik (2014) taucht an mehreren Stellen das Wort „bildungstheoretisch“ auf. Die Frage ist, was in der Berufs- und Wirtschaftspädagogik damit gemeint ist. Die klassische berufsbildungstheoretische Grundidee, dass Bildung über den Weg von Arbeit und Beruf erfolgen kann, wurde in der neuhumanistischen Bildungstheorie angezweifelt, da die Frage nach der bildenden Kraft des Berufs immer auch eine Frage nach der Beschränkung des Menschen und der Enge von Berufsarbeit, an die er gebunden ist, war (vgl. Stratmann 1979/1999, 524). Die Skepsis gegenüber einer Bildung, die das NS-Regime nicht verhindern konnte und für die nun alle Voraussetzungen zerstört waren (vgl. Litt 1947, $12 \mathrm{f}$.), und gleichzeitig der Anspruch, die Berufs- und Arbeitswirklichkeit in der Berufsbildungsdiskussion nicht mehr zu ignorieren, führten nach 1945 dazu, sich entweder ganz vom Bildungsbegriff zu verabschieden oder ihn unter gesellschaftskritischem Aspekt zu begreifen. So war Abel (1963) der Ansicht, dass Bildung durch den Beruf „kein Zauberwert zur Rettung der Menschen“ (S. 196) mehr sei, während für Wilhelm $(1966,97)$ „das Schema ,Bildung durch den Beruf" nicht mehr glaubwürdig" war. Blankertz $(1967 / 75,296)$ räumte ein, dass über Bildung nur noch unter kritischem Vorbehalt gedacht werden könnte und die Berufsbildungstheorie ohnehin „durch den Wandel der Ausbildungsbedingungen beeinträchtigt wird“. Die Rettung der Bildung bei Blankertz erfolgte dann mit dem Hinweis, dass die Begrenzungen von Bildung sie wiederum dazu ermächtigen, ,unter neuen Bedin- 
gungen als organisierende Kraft die Ausbildungskonzeption pädagogisch zu strukturieren" (ebd.), ein Gedanke, der für den bildungstheoretischen Strukturgitteransatz maßgeblich war. Von dem bildungstheoretischen und -kritischen Diskurs dieser Zeit grenzten sich jene Positionen ab, die sich mehr als kognitiv-pragmatisch oder empirisch-analytisch verstehen wollten und auch eher den Erziehungsbegriff bevorzugten. Wie in Teilen der Erziehungswissenschaft wurde auch in der Berufs- und Wirtschaftspädagogik der Erziehungsbegriff als „Oberbegriff mit systembildender Bedeutung“ (Dörschel 1960, 35) favorisiert, dem ,andere erziehungswissenschaftliche Begriffe wie Bildung, Führung, Zucht, Pflege, Berufserziehung, -bildung und -ausbildung ausdrücklich untergeordnet" wurden. In den folgenden Jahrzehnten ist der Bildungsbegriff in der Berufs- und Wirtschaftschaftspädagogik immer mehr in den Hintergrund getreten. Damit sind auch berufsbildungstheoretische Diskurse „aus der Mode gekommen“ (Kutscha 2009, 14). Dies mag auch der Grund dafür sein, dass in berufs- und wirtschaftspädagogischen Einführungsschriften zur Didaktik, „die Rezeption differenzierter [Bildungs] theorien“ abbricht, „,bevor sie konkret (oder ,praktisch') werden könnte“ (Greb 2009, 21).

Eine wesentliche Bezugsgröße bildungstheoretischer Wiederbelebung könnte das Subjektverständnis sein, zumal universelle Form und Zielperspektive verschiedener berufs- und wirtschaftspädagogischer Bemühungen das souveräne Subjekt ist, das autonom Entscheidungen trifft und sich selbst entfaltet. Trotz des bildungstheoretischen Rückzugs ist der Subjektbegriff in der Berufs- und Wirtschaftspädagogik also nach wie vor beliebt, jedoch weniger auf einer theoretisch-analytischen, sondern vielmehr auf einer normativen Ebene. Thoma (2011) arbeitete in „Entwürfe des wirtschaftspädagogischen Subjekts" heraus, dass unklar bleibt, wie das Subjekt konzeptualisiert ist, und wie es durch berufs- und wirtschaftspädagogisch wohl gemeinte subjektorientierte Programme erneut in Machtzentren eingespannt wird. Die im Subjektbegriff liegenden Spannungen und Widersprüche werden in Didaktik- und Kompetenzkonzepten kaum thematisiert. Dabei liegt das, „was ein Subjekt ist, [...] nicht ein für alle Mal fest, sondern lässt sich nur erschließen über die historischen Semantiken und Wissenskomplexe, die Selbst- und Sozialtechnologien, die zu seiner theoretischen Bestimmung und praktischen Formung aufgerufen wurden und werden“ (Bröckling 2007, 23). Somit erweist sich auch die Gegenüberstellung von objektiven Anforderungen auf der einen Seite und Subjekt auf der anderen Seite als zu einfach. In den ,individuellen Bildungsbedürfnissen [kommen] auf so vielfältige und nachhaltige Weise gesellschaftliche, ökonomische und am Ende eben auch betriebliche Anforderungen zur Geltung [...], ja sie sind geradezu inkorporiert, so daß die pädagogisch-idealistische Kontrastierung zwischen [...] ökonomischen Anforderungen einerseits und individuellen Bildungsbedürfnissen andererseits unrealistisch, um nicht zu sagen, als ideologisch interpretiert werden kann“" (Heid 1998, 45). Deshalb geht es in der bildungstheoreti- 
schen Reflexion von Subjekt auch um die Frage, wie Lernende mit wirtschaftlichen, politischen und gesellschaftlichen Denkformen verwoben sind, wie sich diese als Orientierungen in Lebenswelten, „mentalen Infrastrukturen“ (Welzer 2011) und subjektiven Bewertungs- und Verteilungsmaßstäben niederschlagen. Eine Wiederbelebung und Modernisierung des bildungstheoretischen Diskurses könnte dies thematisieren, ebenso die Frage, inwiefern die Disziplin selber von Denkformen gesteuert ist, die sich als Machtformen in ihre subjektorientierten Konzepte und Messforschung einschleichen.

\section{Fazit}

Anliegen des Beitrags ist die Wiederbelebung des Selbstverständnisdiskurses in der Berufs- und Wirtschaftspädagogik. Mögliche Anknüpfungspunkte hierfür können der historische Verlauf der Selbstverständnisfrage, die darin kontinuierlich thematisierten neuralgischen Punkte sowie die Spannungen und Widersprüche disziplinärer Grundkategorien sein.

\section{Literatur}

Abel, H. (1963). Das Berufsproblem im gewerblichen Ausbildungs- und Schulwesen. Braunschweig: Westermann.

Abraham, K. (1953). Die Erziehung des Menschen in der Wirtschaft. In Die Deutsche Berufs- und Fachschule, 49, 321-329.

Abraham, K. (1957). Die Bedeutung der begrifflichen Klarheit für die wirtschaftspädagogische ForschungIn Die Deutsche Berufs- und Fachschule, 53, 169-175.

Albert, H. (1991). Traktat über kritische Vernunft, 5. Auflage, Stuttgart: UTB.

Bank, V. (2009): Berufs- und Wirtschaftspädagogik: Epitaph einer Disziplinlosen. bwp@ Berufs- und Wirtschaftspädagogik - online, 16, 1-22. Online: http://www. bwpat.de/ausgabe 16/bank_bwpat16.pdf (27-12-2016).

Baumert, J. \& Tillmann, J. (2017). Empirische Bildungsforschung. Zeitschrift für Erziehungswissenschaft- Sonderheft 31. Wiesbaden: Springer VS.

Baumgardt, J. (1976). Entwicklung und Stand der Wirtschafts- und Berufspädagogik. In Schanz, H. (Hrsg), Entwicklung und Stand der Berufs- und Wirtschaftspädagogik (S. 7-38). Stuttgart: Holland \& Josenhans.

Beck, K. (2003). Erkenntnis und Erfahrung im Verhältnis zu steuerung und Gestaltung. Zeitschrift für Berufs- und Wirtschaftspädagogik, 99, 232-250.

Bellmann, J. (2001). Knappheit als Bildungsproblem. Weinheim: Deutscher Studienverlag.

Blankertz, H. (1967). Berufsbildungstheorie und berufliche Ausbildungskonzeption. In K. Stratmann \& W. Bartel (Hsrg.). a.a.O. Wiederabdruck (S. 285-299). 
Bröckling, U. (2007). Das unternehmerische Selbst. Soziologie einer Subjektivierungsform. Frankfurt a. M.: Suhrkamp.

Dammer, K.-H. (2015). Vermessene Bildungsforschung. Wissenschaftsgeschichtliche Hintergründe zu einem neoliberalen Herrschaftsinstrument. Baltmannsweiler: Schneider.

Deutsche Forschungsgemeinschaft (Hrsg.) (1990). Berufsbildungsforschung an den Hochschulen der Bundesrepublik Deutschland. Denkschrift. Weinheim: Beltz.

Dörschel, A. (1960). Einführung in die Wirtschaftspädagogik. Berlin und Frankfurt a.M.: Franz Vahlen GmbH.

Feld, F. (1928). Grundfragen der Berufsschul- und Wirtschaftspädagogik. Versuch einer Systematik der berufspädagogischen Theorie. Langensalza: Julius Beltz.

Fischer, A. (1932). Wirtschaft und Schule. In H. Röhrs (Hrsg) (1963). Die Bildungsfrage in der modernen Arbeitswelt. Wiederabdruck (S. 35-60). Frankfurt am Main: Akademische Verlagsgesellschaft.

Geck, L.H.A. (1932). Zur Grundlegung der Wirtschaftspädagogik. Zeitschrift für Handelsschulpädagogik, 4, 145-158 und 220-231.

Greb, U. (2009): Der Bildungsbegriff in einführenden Schriften zur Didaktik der Berufs und Wirtschaftspädagogik.bwp@Berufs- und Wirtschaftspädagogik online, 16, 1-23. Online: http://www.bwpat.de/ausgabe16/greb_bwpat16.pdf (2712-2016).

Groothoff, H.H. (1961). Allgemeine und Wirtschaftspädagogik. Die Deutsche Berufsund Fachschule, 57 (10), 772-781.

Heid, H. (1977). Können „die Anforderungen der Arbeitswelt“ Ableitungsvoraussetzungen für Maßgaben der Berufserziehung sein? Die Deutsche Berufs- und Fachschule, 73 (11), 833-839.

Heid, H. (1998): Berufliche Bildung im Spannungsfeld zwischen betrieblichen Anforderungen und individuellen Ansprüchen. In Berufliches Lernen im Wandel - Konsequenzen für die Lernorte? Dokumentation des 3. Forums Berufsbildungsforschung (33-54). Nürnberg: MitAB.

Kautz, H. (1929). Industrie formt Menschen. Einsiedeln: Verlagsanstalt Benzinger \& Co.

Kell, A. (2014). Grenzgänge, Traditionen und Zukünfte in der Deutschen Gesellschaft für Erziehungswissenschaft. Erziehungswissenschaft. Mitteilungen der DGfE, 25 (49), 49-64.

Kell, A. (2015). Arbeit und Beruf aus Sicht ökologischer Berufsbildungswissenschaft. bwp@Berufs- und Wirtschaftspädagogik - online, Ausgabe 29, 1-25. Online: http://www.bwpat.de/ausgabe29/kell_beitrag1_bwpat29.pdf(27-12-2016).

Kiehn, L. (1962). Gedanken zur wissenschaftstheoretischen Grundlegung der Wirtschafts- und Berufspädagogik. In H. Röhrs (Hrsg.) (1967). Die Wirtschaftspädagogik - eine erziehungswissenschaftliche Disziplin? Wiederabdruck (S. 36-43). Frankfurt a.M.: Akademische Verlagsgesellschaft.

Kipp, M. \& Miller-Kipp, G. (1994). Kontinuierliche Karrieren. Diskontinuierliches Denken? Zeitschrift für Pädagogik, 40 (5), 727-744.

Krasensky, H. (1935). Grundzüge der Wirtschaftspädagogik. Wien: Heymann Verlag.

Kutscha, G. (1992). ,Entberuflichung' und ,Neue Beruflichkeit'. Zeitschrift für Berufs- und Wirtschaftspädagogik, 88, 535-548.

Kutscha, G. (2009). Bildung im Medium des Berufs? In I. Lisop \& A. Schlüter (Hrsg.), a.a.O. (S. 13-36). 
Kutscha, G. (2015). Erweiterte moderne Beruflichkeit. bwp@ Berufs- und Wirtschaftspädagogik - online, 29, 1-22. Online: http://www.bwpat.de/ausgabe29/ kutscha_bwpat29.pdf (27-12-2016).

Kutscha, G. (1976). Das politisch-ökonomische Curriculum. Kronberg/Ts: Fischer Taschenbuch.

Lange, H. (1971). Technik und Praxis. Bemerkungen zum wissenschaftstheoretischen und politischen Standort einer zeitgemäßen Berufs- und Wirtschaftspädagogik. In K. Stratmann \& W. Bartel (Hrsg.). a.a.O. Wiederabdruck (S. 308-337).

Lempert, W. (1972). Vorüberlegungen zum theoretischen Rahmen wissenschaftlicher Analysen beruflicher Bildungsprozesse. In K. Stratmann \& W. Bartel (Hrsg.) a.a.O. (338-349).

Lempert, W. (2009). Die Fliege im Fliegenglas, der Globus von Deutschland und die Berufsbildung ohne Beruf.bwp@Berufs-und Wirtschaftspädagogik-online, 16, 1-45. Online: http://www.bwpat.de/ausgabe16/lempert_bwpat16.pdf (17-122016).

Linke, W. (1957). Gegenstand und Standort der wirtschaftspädagogischen Forschung. Die Deutsche Berufs- und Fachschule, 53 (10), 741-753.

Linke, W. (1961). Kritische Beiträge zur Wirtschaftspäsagogik. Die Deutsche Berufsund Fachschule, 57 (10), 737-738.

Linke, W. (1965). Pädagogischer Erfahrungsaustausch in der praktischen und theoretischen Berufserziehung. Die Deutsche Berufs- und Fachschule, 69 (5),326.

Lipsmeier, A. (1972). Vom Beruf der Berufspädagogen. Zur Wissenschaftstheorie der Berufspädagogik. Die Deutsche Berufs- und Fachschule, 68 (1), 21-49.

Lisop, A. \& Schlüter, A. (Hrsg.) (2009). Bildung im Medium des Berufs? Diskurslinien der Berufs- und Wirtschaftspädagogik. Frankfurt a. M.: G.A.F.B.-Verlag.

Lisop, I. (2009): Identität und Krisenanfälligkeit der Berufs- und Wirtschaftspädagogik.bwp@Berufs-und Wirtschaftspädagogik - online, 16, 1-18. Online: http:// www.bwpat.de/ausgabe16/lisop_bwpat16.pdf (27-12-2016).

Neumann, G. (1969). Die Indoktrination des Nationalsozialismus in der Berufserziehung. Hamburg: Universität Hamburg.

Pleiß, U. (1971). Friedrich Feld und die Begründung der Wirtschaftspädagogik. In Pleiß, U. (Hrsg) (1982), Wirtschaftspädagogik, Bildungsforschung, Arbeitslehre. Wiederabdruck (S. 195-208). Heidelberg: esprint Verlag.

Pleiß, U. (1973). Wirtschaftslehrerbildung und Wirtschaftspädagogik. Göttingen: Schwartz.

Rauner, F. (1985). Technik und Bildung. In Diskurs. Bremer Beiträge zu Wissenschaft und Gesellschaft (S. 110-131), Band 10. Universität Bremen.

Reetz, L. (1976). Beruf und Wissenschaft als organisierende Prinzipien des wirtschaftslehre-Curriculums. Die Deutsche Berufs- und Fachschule, 72 (11), 803818.

Reinisch, H. (2009): Über Nutzen und Schaden des Philosophierens über das Selbstverständnis der Berufs- und Wirtschaftspädagogik. bwp@, Berufs- und Wirtschaftspädagogik - online, 16, 1-17. Online: http://www.bwpat.de/ausgabe16/ reinisch_bwpat16.pdf (27-12-2016).

Ritzel, W. (1961). Aktuelle Fragen der Wirtschaftspädagogik und des wirtschaftspädagogischen Studiums. Die Deutsche Berufs- und Fachschule, 57 (10), 738-753.

Schlieper, F. (1949). Die Lehre von der Wirtschaftserziehung. Die Deutsche Berufsund Fachschule, 45 (1), 42. 
Schlieper, F. (1952). Wesen und Inhalt einer Lehre von der Wirtschaftserziehung. Die Deutsche Berufs- und Fachschule, 48 (5), 423.

Schlieper, F. (1954). Grundbegriffe der Wirtschaftspädagogik. In H. Röhrs (Hrsg.) (1967). Die Wirtschaftspädagogik - eine erziehungswissenschaftliche Disziplin? (S. 54-74). Wiederabdruck. Frankfurt a.M.: Akademische Verlagsgesellschaft.

Schlieper, F. (1963). Allgemeine Berufspädagogik. Freiburg im Breisgau: Lambertus.

Schmiel, M.; Sommer, K.-H. (2001). Berufs- und Wirtschaftspädagogik als wissenschaftliche Disziplin. In H. Schanz (Hrsg.). Berufs- und wirtschaftspädagogische Grundprobleme (S. 8-21). Baltmannsweiler: Schneider.

Schürholz, F. (1928). Grundlagen einer Wirtschaftspädagogik. Zum Kampf um Wirtschaftsführung und Sozialordnung. Erfurt: K. Stenger.

Schwarzlose, A. (1961). Empirische Forschung in der Wirtschaftspädagogik. Die Deutsche Berufs- und Fachschule, 57 (10), 753-768.

Seubert, R. (1977). Berufserziehung und Nationalsozialismus. Weinheim: Beltz.

Stratmann, J. (1979). Berufs- und Wirtschaftspädagogik. In G. Pätzold \& Wahle, M. (Hrsg.) (1999), Karlwilhelm Stratmann. Berufserziehung und sozialer Wandel. Wiederabdruck (S. 509-581). Frankfurt a.M.: Gesellschaft zur Förderung arbeitsorientierter Forschung und Bildung Verlag.

Stratmann, K. \& Bartel, W. (Hrsg.) (1975). Berufspädagogik. Ansätze zu ihrer Grundlegung und Differenzierung. Köln: Kiepenheuer \& Witsch.

Stratmann, K. (1972). Zum Thema dieses Heftes: Das Theorie-Praxis-Verhältnis in der Berufs- und Wirtschaftspädagogik. Die Deutsche Berufs- und Fachschule, 68 (8), 575-577.

Stratmann, K. (1975). Historische Pädagogik als Mittel der Entmythologisierung und Entideologisierung. In J.L. Blaß u. a. (Hrsg.), Bildungstradition und moderne Gesellschaft, (S. 304-322). Hannover: Schrödel.

Tenorth, H.E. (2004). Erziehungswissenschaft. In D. Benner \& J. Oelkers (Hrsg.). Historisches Wörterbuch der Pädagogik (S. 341-382). Weinheim: Beltz.

Thoma, M. (2011). Entwürfe eines wirtschaftspädagogischen Subjekts. Wiesbaden: VS Verlag.

Weingart, P. (1976): Wissensproduktion und soziale Struktur. Frankfurt am Main: Suhrkamp.

Weingart, P. (2003). Wissenschaftssoziologie. Bielefeld: transcript.

Welzer, H. (2011). Mentale Infrastrukturen. Heinrich Böll Stiftung. Online: https:// www.boell.de/sites/default/files/Endf_Mentale_Infrastrukturen.pdf (20-12-2016).

Wilhelm, T. (1966). Das Arbeitsethos der Gegenwart im Lichte der deutschen Bildungsüberlieferung. In K. Stratmann \& W. Bartel (Hrsg.) (1975), a.a.O., Wiederabdruck (S. 95-111).

Zabeck, J. (1968). Zur Grundlegung und Konzeption einer Didaktik der kaufmännischen Berufserziehung. In Jahrbuch für Wirtschafts- und Sozialpädagogik (S. 87141). Heidelberg: Quelle \& Meyer.

Zabeck, J. (1972). Das Theorie-Praxis-Problem in der Wirtschaftspädagogik. Die Deutsche Berufs- und Fachschule, 68 (8), 577-599. 


\title{
Orientierungspotenziale des Basiscurriculums der Berufs- und Wirtschaftspädagogik für die disziplinäre Binnen- und Außenlegitimität
}

\author{
Matthias Söll
}

\section{Einleitung}

Der 1999 initiierte Bologna-Prozess zielt auf die Entwicklung transparenter und vergleichbarer Studiengänge $a b$, die in einem einheitlichen europäischen Hochschulraum eine hohe Studierendenmobilität begünstigen sollen (vgl. o.V. 1999, 1 ff.). Um die Qualität der tertiären Bildungsgänge hinsichtlich dieser Maßstäbe zu sichern und zu entwickeln, werden zahlreiche formelle Vorgaben erlassen. Zur Gewährleistung deren Umsetzung erfolgt zudem die Etablierung eines Akkreditierungssystems (vgl. KMK 2002, 4 ff.).

Die erziehungswissenschaftliche Teildisziplin Berufs- und Wirtschaftspädagogik konkretisiert die Richtlinien der Hochschulreformen, indem sie Standards für das von ihr verantwortete Studienfach erarbeitet. Im Basiscurriculum der Sektion Berufs- und Wirtschaftspädagogik der Deutschen Gesellschaft für Erziehungswissenschaft (Sektion BWP der DGfE) gibt die Disziplin an, dass sie sich bei der Studiengangentwicklung an den Grundsätzen des Bologna-Prozesses orientiert: „Mit dem Basiscurriculum verbindet sich [...] die Erwartung, dass lokale Lehrprogramme auf einen einheitlichen Rahmen bezogen werden und in ihren wesentlichen Inhalten übereinstimmen" (Sektion BWP der DGfE 2014, 6). Das Statut wurde im Jahr 2003 veröffentlicht und im Jahr 2014 aktualisiert (vgl. Sektion BWP der DGfE 2003, 2014).

Vor diesem Hintergrund steht die Entwicklung von Studiengängen regelmäßig im Fokus hochschulpolitischer Diskurse. Diese werden allerdings selten auf Basis empirischer Forschungsarbeiten geführt, sodass sie meist normativ oder subjektiv geprägt sind (vgl. Zlatkin-Troitschanskaia \& Kuhn 2010, 1). Pätzold (2012, 12 ff.) konstatiert, dass dies auch für die Studiengänge der Berufs- und Wirtschaftspädagogik gilt.

Um eine Grundlage zur empiriegestützen Entwicklung wirtschaftspädagogischer Studiengänge zu schaffen, wurde im Rahmen einer Curriculumanalyse eine entsprechende Vollerhebung durchgeführt. Die Untersuchung der Kernelemente der 113 ermittelten, profilierten Bachelor- und Masterstudiengänge an 30 Universitätsstandorten zeigt, dass sich deren Ziele zwar ähneln, ihre Strukturen und Inhalte allerdings teils erheblich voneinander sowie von den Vorgaben der Studiengangentwicklung abweichen (vgl. Söll 
2016, 266 ff.). Für konsekutive Studiengänge ist im Basiscurriculum bspw. ein Umfang von 75 Leistungspunkten (LP) für die Inhalte der Berufs- und Wirtschaftspädagogik vorgesehen (vgl. Sektion BWP der DGfE 2014, 12). Diese Norm erreichen derzeit 65,2 \% der untersuchten Bildungsgänge (vgl. Söll 2016, 264). Im Kontext der skizzierten Rahmenbedingungen der Entwicklung von Studiengängen sollte die stark eingeschränkte Transparenz und Vergleichbarkeit überraschen, zumal $76,3 \%$ der wirtschaftspädagogischen Studiengänge bereits erfolgreich akkreditiert wurden (vgl. Söll 2016, 94).

Während die Institutionen und Organisationen, die formelle Vorgaben zur Studiengangentwicklung erlassen, insbesondere die Erhöhung der Qualität und Kompatibilität von Bildungsprozessen und -abschlüssen anvisieren, fokussieren wissenschaftliche Disziplinen darüber hinaus auch auf deren Profilierung (vgl. Brand \& Tramm 2002, 266 f.). So setzt sich die Berufsund Wirtschaftspädagogik im Zuge der Formulierung von Studienstandards auch mit ihrem disziplinären Selbstverständnis auseinander (vgl. Sektion BWP der DGfE 2003, 1f. sowie 2014, 1 f.). Aus wissenschaftssoziologischer Perspektive dienen diese Debatten und ihre Ergebnisse der Konstituierung einer starken disziplinären Binnen- und Außenlegitimität (vgl. Lepsius 1973, 105 ff.).

Im Hinblick auf die Entwicklung des heterogenen Systems wirtschaftspädagogischer Studiengänge ist im vorliegenden Aufsatz die Frage handlungsleitend, welche Orientierungspotenziale die Genese des Basiscurriculums für das Innen- und Außenverhältnis der Disziplin Berufs- und Wirtschaftspädagogik bietet. Den Ausgangspunkt der Darstellungen bilden die vielschichtigen Rahmenbedingungen der Studiengangentwicklung, die in Kapitel 2 systematisiert werden. Im Zentrum des Beitrags stehen in Kapitel 3 dann die Entwicklung, die Ziele, die Orientierungsrahmen und der Aufbau der Basiscurricula sowie deren Regelungen zu den Zielen, Strukturen und Inhalten berufs- und wirtschaftspädagogischer Studiengänge. In Kapitel 4 schließlich werden die Orientierungspotenziale der Implementation der Basiscurricula der Berufs- und Wirtschaftspädagogik für die disziplinäre Binnen- und Außenlegitimität diskutiert.

\section{Formelle Rahmenbedingungen der Studiengangentwicklung}

Diplom- und Staatsexamensstudiengänge basierten auf staatlich genehmigten Rahmenordnungen und Vorgaben. Für die Entwicklung von Bachelor- und Masterstudiengängen ist gemäß der Grundsätze des New Public Managements das teil- bzw. nichtstaatliche Akkreditierungssystem verantwortlich (vgl. KMK 2002, 5; Wolter \& Kerst 2008, 137 ff.). Bisher wurde für die 
Entwicklung von Studiengängen allerdings kein einheitliches Verfahren eingeführt. Den Ausgangspunkt entsprechender Leitfäden, bspw. „Zehn Schritte nach ,Bologna“" (vgl. HRK 2004, $10 \mathrm{ff}$ ), bildet die Sichtung von formellen Rahmenbedingungen der Studiengangentwicklung.

Zur Entwicklung berufs- und wirtschaftspädagogischer Studiengänge liegt eine aktuelle, umfassende Übersicht mit Analyse der relevanten Vorgaben vor (vgl. Söll 2016, 79 ff. und 133 ff.). Diese geht von den Landeshochschulgesetzen als rechtlichen Basen der Studiengangentwicklung aus. Demnach werden fachspezifische Studiengänge wie die der Betriebswirtschaftslehre oder der Mathematik von (1.) den Landeshochschulgesetzen, (2.) den ländergemeinsamen Strukturvorgaben für die Akkreditierung, (3.) deren Auslegung durch den Akkreditierungsrat, (4.) den landesspezifischen Strukturvorgaben für die Akkreditierung, (5.) den Regeln für die Akkreditierung, (6.) den Vorgaben der Akkreditierungsagenturen und (7.) dem Qualifikationsrahmen für Deutsche Hochschulabschlüsse geregelt. Zudem gelten für lehramtsspezifische Studiengänge (1.) rechtliche Regelungen der Bundesländer, ${ }^{1}$ (2.) die Rahmenvereinbarung über die Ausbildung und Prüfung für ein Lehramt der Sekundarstufe II (berufliche Fächer) oder für die beruflichen Schulen (Lehramtstyp 5), (3.) die Standards für die Lehrerbildung in den Bildungswissenschaften und (4.) die ländergemeinsamen inhaltlichen Anforderungen für die Fachwissenschaften und Fachdidaktiken. Bei der Konzeption berufs- und wirtschaftspädagogischer Bildungsgänge fordert die Sektion BWP der DGfE $(2014,3)$ darüber hinaus die Berücksichtigung des rechtlich nicht bindenden Basiscurriculums.

Das Ergebnis der Recherche zeigt zunächst, dass bei der Studiengangentwicklung eine Vielzahl an Vorgaben zu berücksichtigen ist. Diese werden von den gesetzgebenden Instanzen des Bundes und der Länder sowie der Kultusministerkonferenz (KMK), der Hochschulrektorenkonferenz (HRK), dem Wissenschaftsrat und dem Akkreditierungsrat erlassen. Die Analyse der Dokumente in Bezug auf die Regelungen der Ziele, Strukturen, Inhalte und Rahmenbedingungen der Studiengänge zeichnet ein äußerst heterogenes Bild. Zwar können in den Vorgaben, die unterschiedliche Schwerpunkte setzen, grundsätzliche Gemeinsamkeiten in Bezug auf die Festlegung der Konturen des Studiums identifiziert werden, jedoch finden sich auch zahlreiche widersprüchliche Regelungen, bspw. zur strukturellen und inhaltlichen Gestaltung. Auch die Konzepte, auf denen die Vorgaben basieren, bspw. das Kompetenzkonzept, unterscheiden sich zum Teil voneinander.

1 Von den 14 Bundesländern, in denen bspw. das Studium der Wirtschaftspädagogik angeboten wird, haben sieben Lehrerbildungsgesetze und zwei spezifische Verordnungen erlassen. In fünf Bundesländern werden die Studiengänge ohne landesrechtliche Vorgaben konzipiert. Nach Walm und Wittek $(2014,16)$ zeigt sich daran die unterschiedliche Bedeutung, welche die Bundesländer der Lehrerbildung zuschreiben. 
Die Vorgaben zu fachspezifischen Studiengängen beinhalten keine fachlichinhaltlichen Standards, da diese bei der Akkreditierung im Rahmen von PeerReview-Verfahren durch geeignete Gutachter zu prüfen sind. Dies steht im Einklang mit dem durch das New Public Management forcierten Paradigmenwechsel von einer Input- zu einer Outcome-Steuerung (vgl. KMK 2002, 5; Wildt 2007, 44 f.). Dagegen finden sich in den Richtlinien zu lehramtsspezifischen Studiengängen zahlreiche Regelungen zu Studieninhalten. Überdies entsenden die für diese Bildungsgänge verantwortlichen Ministerien häufig Staatsvertreter mit Vetorecht in die Gutachtergruppen der Akkreditierungsverfahren. Somit wird das Ziel der staatlichen Deregulierung der Entwicklung von Studiengängen des Lehramts konterkariert (vgl. Reuter 2007, 124). Insgesamt ist davon auszugehen, dass sich dieses unsystematische Geflecht an Vorgaben eher hemmend als fördernd auf die Orientierung bei der Entwicklung transparenter und vergleichbarer Studiengänge auswirkt.

\section{Basiscurriculum für das universitäre Studienfach Berufs- und Wirtschaftspädagogik}

Die Basiscurricula für das universitäre Studienfach Berufs- und Wirtschaftspädagogik dienen einerseits der Steigerung der Qualität und Kompatibilität der entsprechenden Studienprozesse und -abschlüsse. Andererseits fokussiert die Berufs- und Wirtschaftspädagogik auch auf die Schärfung des disziplinären Profils. Im Folgenden werden die Genese, die Intentionen, die Leitgedanken und das Grundgerüst der Basiscurricula sowie deren Regelungen zu den curricularen Kernelementen berufs- und wirtschaftspädagogischer Studiengänge untersucht. Dabei steht die Frage im Mittelpunkt, inwiefern die Entwicklung der Richtlinie zur Konstituierung einer starken disziplinären Binnen- und Außenlegitimität beitragen kann.

\subsection{Genese}

Neben den Studienstrukturen stand an der Wende zum 21. Jahrhundert auch die inhaltliche Ausrichtung von Studiengängen zur Diskussion. Die Implementation eines Kerncurriculums wurde bspw. vom Wissenschaftsrat (2001, 29) als eine zentrale Reformaufgabe in der Lehrerbildung ausgewiesen. Kernoder Basiscurricula können als Ergebnisse der Kanonisierung von Bildung betrachtet werden (vgl. Tenorth 2004, 651 ff.). Zahlreiche Erziehungswissenschaftlerinnen und Erziehungswissenschaftler debattierten in dieser Zeit intensiv über ein solches Instrument (vgl. bspw. die Beiträge in der ,Zeitschrift für Pädagogik“ 45 [1999] 5 und in der Zeitschrift „Erziehungswissen- 
schaft" 15 [2004] 28). Auch die Vertreterinnen und Vertreter der Berufs- und Wirtschaftspädagogik beschäftigten sich mit dieser Thematik, wenngleich sich entsprechende Positionierungen seltener in der Literatur niederschlugen (vgl. Buchmann \& Kell 2001, 96 f.; Beck 2002, 124 f.).

Beck $(2002,125$ f.) legt die Funktion eines Kerncurriculums für Lehrerinnen und Lehrer an berufsbildenden Schulen aus professionssoziologischer Perspektive offen. Die Lehrenden erbringen als Professionsangehörige eine spezifische gesellschaftliche Leistung. Dabei greifen sie auf einen gemeinsamen Wissensbestand zurück. Dessen „Kern“ muss in der Lehrerbildung durch ein entsprechendes „Curriculum“ - ein Kerncurriculum - entwickelt und gesichert werden.

Das unter der Federführung von Beck und Zabeck erarbeitete Basiscurriculum für das universitäre Studienfach Berufs- und Wirtschaftspädagogik im Folgenden auch 1. Basiscurriculum - wurde im Jahr 2003 verabschiedet (vgl. Sektion BWP der DGfE 2003, 1; Sloane 2003, 490). ${ }^{2}$ Gut zehn Jahre später erfolgte die Bewilligung des durch Weber, Kremer, Tenberg, Weyland und Beck überarbeiteten Grundsatzpapiers - im Folgenden auch 2. Basiscurriculum (vgl. Sektion BWP der DGfE 2014, 2 ff.).

Die Curricula beruhen auf intradisziplinären, öffentlichen Diskursen zwischen den Fachvertreterinnen und Fachvertretern. Darüber hinaus wurden die berufs- und wirtschaftspädagogische Lehre und Forschung, die rechtlichen Rahmenbedingungen der Studiengänge sowie die Anforderungen von Tätigkeiten, die Absolventinnen und Absolventen nach dem Studium in der Regel ausführen, analysiert (Sektion BWP der DGfE 2003, 1 ff. sowie 2014, 1 ff.).

\subsection{Ziele}

Grundsätzlich markieren die Curricula Eckpunkte des disziplinären Selbstverständnisses und setzen inhaltliche Standards (Sektion BWP der DGfE 2003, 1 f. sowie 2014, 1 f.). Durch die Verständigung auf eine gemeinsame Identität zielen die Richtlinien bezüglich des disziplinären Innenverhältnisses einerseits auf die Professionalisierung der Berufs- und Wirtschaftspädagogik selbst ab. Andererseits sichern und entwickeln sie hinsichtlich des disziplinären Außenverhältnisses durch die Vorgabe inhaltlicher Standards die Qualität des universitären Studiums der Berufs- und Wirtschaftspädagogik, das die Professionalität der Absolventinnen und Absolventen entwickeln soll.

2 Das Basiscurriculum wurde anfangs auch als Kerncurriculum bezeichnet (vgl. Bank 2009, 4; Sloane 2003, 490). Die DGfE veröffentlichte im Jahr 2008 allerdings selbst ein Kerncurriculum für erziehungswissenschaftliche Haupt- und Nebenfachstudiengänge sowie für Lehramtsstudiengänge (vgl. DGfE 2010, 7). Die Differenzierung der Bezeichnungen soll einer Verwechslung vorbeugen. 


\subsection{Orientierungsrahmen}

Beck (2005, 5 und 10) plädiert ausdrücklich für ein professionalitätsförderndes, input-orientiertes Basiscurriculum. In einem Brückenschlag zwischen Input- und Outcome-Orientierung sieht der Autor die Gefahr der Marginalisierung von Inhalten. Aufgrund der häufig fehlenden Forschungsergebnisse über die Zusammenhänge zwischen Input und Outcome könne eine Systematisierung, bspw. in einer Matrix, nur spekulativ erfolgen. Daraus resultiere die Gefahr der systematischen Unter- oder Überschätzung des Geltungsanspruchs der Inhalte. Dies führe bezüglich der Entwicklung berufs- und wirtschaftspädagogischer Professionalität zu Qualitätseinbußen (vgl. Beck 2005, 14 f.).

Sloane (2003, 490 f.) und Wilbers (2004, 11 f.) machten dagegen bereits zur Zeit der Einführung des Basiscurriculums auf die Problematik aufmerksam, dass lehrerbildende Studiengänge vor dem Hintergrund der vorherrschenden Bildungs- und Hochschulpolitik standard- und kompetenzbasiert gestaltet werden sollten. Als Lösung schlägt Sloane (2003, 490 f.) die von Beck (2005, 5 und 10) abgelehnte, systematische Zusammenführung der fachwissenschaftlichen Struktur der Berufs- und Wirtschaftspädagogik mit den sich an Outcomes orientierenden Standards der Lehrerbildung vor. Auch Brand und Tramm (2002, 269 ff.) setzen sich für solch eine integrative Lösung ein.

Zur Förderung berufs- und wirtschaftspädagogischer Professionalität rekurriert die erste Fassung des Basiscurriculums auf inhaltliche Standards (vgl. Sektion BWP der DGfE 2003, 1), während die zweite diesbezüglich einen kompetenzorientierten Raum (vgl. Sektion BWP der DGfE 2014, 2) aufspannt. Allerdings wird in dem letztgenannten Statut nicht allein auf die Förderung von Kompetenz fokussiert. Indem auch die zentralen Inhalte der Berufs- und Wirtschaftspädagogik explizit genannt und strukturell verortet werden, haben sie weiterhin ein starkes Gewicht. Somit ist das ursprüngliche Curriculum eher wissenschaftsorientiert, das überarbeitete eher situationsund persönlichkeitsorientiert.

\subsection{Aufbau}

Die beiden Richtlinien ähneln sich hinsichtlich ihres Aufbaus, der in Tabelle 1 veranschaulicht wird. In den Kapiteln 1 und 2 wird in die Basiscurricula eingeführt. Darauf folgt die Formulierung von Leitbildern (Kapitel 3) und Professionalitätsdimensionen (Kapitel 4). Die in den Kapiteln 5 systematisierten inhaltlichen Schwerpunkte der Studiengänge werden allerdings nur im 1. Basiscurriculum in Kapitel 6 spezifiziert. Beide Statute enden mit Implementationsgrundsätzen (1. Basiscurriculum: Kapitel 7; 2. Basiscurriculum: 
Kapitel 6). Die unterschiedlichen Orientierungen der Curricula spiegeln sich auch in den Umfängen der einzelnen Kapitel wider. Im eher wissenschaftsorientierten 1. Basiscurriculum nehmen die Kapitel 5 und 6, die Studieninhalte präzisieren, einen Umfang von 58,6\% ein. ${ }^{3}$ Der mit diesen Kapiteln im 2. Basiscurriculum korrespondierende Teil 5 hat dagegen nur einen Umfang von $12,6 \%$. Themen wie künftige berufliche Tätigkeitsfelder, professionelles Handeln, dafür notwendige Dispositionen und spezifische Kompetenzprofile werden im 1. Basiscurriculum in den Kapiteln 3 und 4 mit einem Umfang von 9,4\% abgedeckt. Diese Gegenstände nehmen im 2. Basiscurriculum in den Kapiteln 3, 4 und 5 dagegen 54,3\% des Gesamtumfangs ein.

Tab. 1: Aufbau der Basiscurricula

\begin{tabular}{|c|c|c|c|c|c|c|c|}
\hline Nr. & $\begin{array}{l}\text { Basiscurriculum } \\
2003\end{array}$ & $\sum \mathbf{Z} .:$ & $\%$ & Nr. & $\begin{array}{l}\text { Basiscurriculum } \\
2014\end{array}$ & $\sum \mathbf{Z} .:$ & $\%$ \\
\hline 1 & Vorwort & 6.391 & 17,5 & 1 & Vorwort & 8.966 & 26,7 \\
\hline 2 & Präambel & 3.957 & 10,8 & 2 & Geltungsbereich & 5.085 & 15,1 \\
\hline 3 & Leitbild & 2.145 & 5,9 & 3 & Leitbild & 4.172 & 12,4 \\
\hline 4 & $\begin{array}{l}\text { Professionalitäts- } \\
\text { dimensionen }\end{array}$ & 1.265 & 3,5 & 4 & $\begin{array}{l}\text { Professionalitäts- } \\
\text { dimensionen }\end{array}$ & 9.840 & 29,3 \\
\hline 5 & Rahmenstruktur & 1.884 & 5,2 & 5 & $\begin{array}{l}\text { Hinweise für } \\
\text { Kompetenzprofile }\end{array}$ & 4.232 & 12,6 \\
\hline 6 & Lehrinhalte & 19.505 & 53,4 & 6 & $\begin{array}{l}\text { Implementations- } \\
\text { grundsätze }\end{array}$ & 1.346 & 4,0 \\
\hline \multirow[t]{2}{*}{7} & $\begin{array}{l}\text { Implementations- } \\
\text { grundsätze }\end{array}$ & 1.393 & 3,8 & & & & \\
\hline & & 36.540 & & & & 33.641 & \\
\hline
\end{tabular}

Z.: Zeichen inklusive Leerzeichen (Erhebung mit Word 2013)

Quelle: Eigene Darstellung

3 In Kapitel 6 werden jedem Themengebiet auf 14 Seiten in tabellarischer, stichpunktartiger Form durchschnittlich 7,9 Unterpunkte (Min=3; $M a x=12 ; S D=2,6$ ), die zum Teil nochmals untergliedert sind, zugeordnet. Das ursprünglich veröffentlichte Kerncurriculum ähnelt mit Ausnahme des Vorworts dem 1. Basiscurriculum. Es wurde allerdings um einen Anhang ergänzt, der zu den Kapiteln 5 und 6 ca. 750 Quellen ausweist. 


\subsection{Studienziele}

Gemäß der Leitbilder der Basiscurricula sollen Absolventinnen und Absolventen des berufs- und wirtschaftspädagogischen Studiums in der Lage sein,

„-- Erkenntnisse im Bereich der Berufs- und Wirtschaftspädagogik theoriegeleitet und systematisch zu erarbeiten,

- praktische Fragen und Probleme in den genannten Tätigkeitsfeldern theoriegeleitet und kritisch zu reflektieren sowie

- theoriegeleitet begründete, auf individuelle und kollektive Bedürfnisse abgestimmte Lösungen zu entwickeln und umzusetzen“ (Sektion BWP der DGfE 2014, 7; vgl. Sektion BWP der DGfE 2003, 6).

Diese professionelle, ethisch-reflexive Handlungskompetenz ist im Sinne lebenslangen Lernens stetig weiterzuentwickeln (vgl. Sektion BWP der DGfE 2014, 7 f. sowie 2003, 6). Es werden zudem typische Tätigkeitsfelder wie das berufsbildende Schulwesen, das betriebliche Bildungs- und Personalwesen oder die berufs- und wirtschaftspädagogische Forschung und Lehre sowie mit diesen korrespondierende professionelle Handlungen wie die Curriculumentwicklung, die Gestaltung von Lehr-Lern-Prozessen oder die Entwicklung der Rahmenbedingungen der beruflichen Bildung systematisiert (Sektion BWP der DGfE 2003, 6 sowie 2014, 6 ff.).

\subsection{Studienstruktur}

Das im Jahr 2003 eingeführte Basiscurriculum bezieht sich auf grundständige Studiengänge mit Diplom- oder Staatsexamensabschlüssen (vgl. Sektion BWP der DGfE 2003, 3 und 26). Für diese wurden insgesamt 160 Semesterwochenstunden (SWS) veranschlagt (vgl. KMK 1999, 22). Dabei soll das Fach Berufs- und Wirtschaftspädagogik inklusive der fachspezifischen Didaktik der jeweiligen beruflichen Fachrichtung ca. 40 SWS umfassen (vgl. Sektion BWP der DGfE 2003, 5). Dies entspricht einem Studienanteil von 25 Prozent.

Die im Rahmen des Bologna-Prozesses eingeführte Studienstruktur bildet den Ausgangspunkt des überarbeiteten Basiscurriculums aus dem Jahr 2014 (vgl. Sektion BWP der DGfE 2014, 3). Bei einem Bachelor- und Masterstudium sind insgesamt 300 LP zu erwerben (vgl. KMK 2010, 3). In dem Grundsatzdokument der Sektion BWP wird für das Fach Berufs- und Wirtschaftspädagogik ein Umfang von ca. 75 LP gefordert (vgl. Sektion BWP der DGfE 2014, 12). Der anvisierte fachspezifische Studienanteil beträgt somit ebenfalls 25 Prozent. 


\subsection{Studieninhalte}

Die Basiscurricula weisen jeweils fünf Studienschwerpunkte mit dazugehörigen Themengebieten aus:

- Didaktische Inhalte (1. Basiscurriculum: „Didaktik der beruflichen Ausund Weiterbildung“" [26,7\%]; 2. Basiscurriculum: „Berufliches Lehren, Lernen, Entwickeln“ [25,0 \%]) nehmen in beiden Curricula ca. 25,0 \% des Fachs ein.

- Auf die Auseinandersetzung mit der Berufspraxis (1. Basiscurriculum: „Unterrichts- und unterweisungspraktische Studien“ [13,3\%]; 2. Basiscurriculum „Berufs- und wirtschaftspädagogische Praxisfelder“ [15,0 $\%$ ]) entfallen jeweils ungefähr 15,0 \% dieses Studienteils.

- Die Schwerpunkte „Grundlagen der Berufs- und Wirtschaftspädagogik“ $(20,0 \%)$ und "Bedingungen und Strukturen beruflichen Lernens“ $(26,7 \%)$ wurden im Zuge der Überarbeitung des Curriculums in ,Theorien, Organisation, Strukturen beruflicher Bildung“ $(25,0 \%)$ und „Professionalisierung" $(10,0 \%)$ umstrukturiert. Dies ging mit einer Reduzierung der Studienanteile von insgesamt $46,7 \%$ auf $35,0 \%$ einher.

- Der Bereich „Forschungsmethoden“ wurde somit von knapp 15,0 \% auf 25,0 \% (1. Basiscurriculum: „Ansätze und Methoden der quantitativen und qualitativen Berufsbildungsforschung “ $[13,3 \%] ; 2$. Basiscurriculum: „Forschungsmethoden“ [25,0\%]) deutlich aufgewertet (vgl. Sektion BWP der DGfE 2003, 8 sowie 2014, 12).

\section{Zusammenfassung und Fazit}

Die Basiscurricula der Berufs- und Wirtschaftspädagogik spiegeln in Bezug auf das korrespondierende Studium zum einen die vorherrschenden Normen, Werte und Einstellungen berufs- und wirtschaftspädagogischer Fachvertreterinnen und Fachvertreter wider. Zum anderen regeln sie die Ziele, Strukturen, Inhalte und Rahmenbedingungen der entsprechenden Studiengänge. Während das erste, eher wissenschaftsorientierte Statut auf die inhaltliche Dimension des Studiums fokussiert, konzentriert sich das zweite, eher situations- und persönlichkeitsorientierte, auf dessen Zieldimension. Abschließend werden die Orientierungspotenziale der Curricula vor dem Hintergrund der Konstituierung der disziplinären Binnen- und Außenlegitimität der Berufs- und Wirtschaftspädagogik diskutiert.

Die Vielzahl an formellen Vorgaben ist für die Entwicklung transparenter und vergleichbarer Studiengänge eher ab- als zuträglich. Bei der Analyse der Inhalte der in Kapitel 2 systematisierten Reglementierungen fällt aller- 
dings auf, dass sich diese überwiegend auf unterrichts- und schulnahe Tätigkeiten beziehen. Das zentrale Prinzip der Polyvalenz berufs- und wirtschaftspädagogischer Bachelor- und Masterstudiengänge ist lediglich in den ländergemeinsamen inhaltlichen Anforderungen für die Fachwissenschaften und Fachdidaktiken verankert. Dagegen war dieser Grundsatz zentraler Bestandteil der Diplomstudiengänge regelnden Rahmenordnung (vgl. Söll 2016, 133 ff.). In Bezug auf die Regelungsebene kann konstatiert werden, dass die Bedeutung des polyvalenten Studiengangmodells zugunsten des lehrerbildenden abgenommen hat (vgl. Söll 2016, 163 f.). Hinsichtlich des disziplinären Außenverhältnisses ist es folglich durchaus sinnvoll, die eigenen Prinzipien in einem konsistenten Basiscurriculum nach außen zu kommunizieren und dessen Berücksichtigung bei der Studiengangentwicklung sowie bei der Akkreditierung einzufordern.

Sloane $(2003,490$ f.) und Wilbers $(2004,15)$ weisen darauf hin, dass die Richtlinie regelmäßig an bildungs- und hochschulpolitische Rahmenbedingungen, wie die gestufte Studienstruktur mit Bachelor- und Masterabschlüssen oder die Outcome-Orientierung, angepasst werden muss. Fehlen entsprechende Bezüge, droht eine partielle Umsetzung des Statuts und damit die Verfehlung der ursprünglichen Ziele. Ferner besteht die Gefahr, dass sich Studiengangentwicklerinnen und -entwickler an generellen Vorgaben orientieren müssen, wenn sie ihre Spezifika nicht in den aktuellen Diskurs einbringen. Das Ergebnis einer explorativ angelegten Interviewstudie zu den Orientierungen von 14 Professorinnen und Professoren der Wirtschaftspädagogik kann als Indiz für diese Befürchtungen interpretiert werden. Es zeigt sich, dass die Befragten das sich auf Diplom- und Staatsexamensstudiengänge beziehende 1. Basiscurriculum bei der Ableitung von Zielen (21,4\%), Strukturen, Rahmenbedingungen $(7,1 \%)$ und Inhalten $(50,0 \%)$ von Bachelor- und Masterstudiengängen nur vereinzelt als Richtlinie nutzten (vgl. Söll 2016, 208 ff.). Hinsichtlich der disziplinären Außenlegitimität rechtfertigen diese Argumente die Implementation der gestuften Studienstruktur sowie der Outcome-Orientierung im überarbeiteten Basiscurriculum.

Bei der Modifikation der Vorgaben muss allerdings berücksichtigt werden, dass deren Orientierungspotenzial bei der Gestaltung von Studiengängen sinken kann, wenn die Beschreibungen der Kernbestandteile der Bildungsgänge einen zu hohen Abstraktionsgrad aufweisen. Diesbezüglich befürchtet Beck (2005, 14 f.), die Entwicklung berufs- und wirtschaftspädagogischer Professionalität werde ,im Kompetenzkorsett verkümmern“ (Beck 2005, 15). Einerseits spricht die Berücksichtigung aktueller bildungs- und hochschulpolitischer Rahmenbedingungen im 2. Basiscurriculum dafür, dass dieses bei der Ableitung von Studienzielen, -strukturen und -rahmenbedingungen mehr Orientierung bietet. Andererseits wirkt sich die starke Reduzierung der Präzisierung von Studieninhalten zugunsten der Beschreibung eines kompetenz- 
orientierten Raums möglicherweise negativ auf das Orientierungsvermögen der Leitlinie aus.

Die Entwicklung von Basiscurricula entfaltet ihre Wirkung auch in Bezug auf das disziplinäre Innenverhältnis. Aushandlungsprozesse zwischen den Vertreterinnen und Vertretern der Berufs- und Wirtschaftspädagogik über die Ziele, Inhalte, Strukturen und Rahmenbedingungen des Studiums kennzeichnen eine lebendige wissenschaftliche Disziplin (vgl. Reinisch 2009, 9 und 13 f.). Dabei müssen die Vorgaben auch hinsichtlich empirischer Erkenntnisse zur Studiengangentwicklung diskutiert werden. So zeigt die Untersuchung der Inhaltsbereiche konsekutiver wirtschaftspädagogischer Studiengänge, dass Forschungsmethoden bspw. durchschnittlich lediglich einen Umfang von 8,8 \% einnehmen (vgl. Söll 2016, 261). Deren Bedeutung ist im aktuellen Basiscurriculum mit einem Studienanteil von 25 Prozent dagegen deutlich größer. ${ }^{4}$

Die sich an entsprechende Diskurse anschließende Einigung auf ein Basiscurriculum als Konsens über den normativen, strukturellen und inhaltlichen Rahmen für berufs- und wirtschaftspädagogische Studiengänge lässt sich als Stärkung der disziplinären Binnenlegitimität interpretieren. Diese könnte noch intensiviert werden, indem die Auseinandersetzungen in einschlägigen Publikationsorganen veröffentlicht würden. Da an der Binnenlegitimität der Disziplin ebenfalls kontinuierlich weitergearbeitet werden muss, ist die Aktualisierung des Basiscurriculums auch aus innerdisziplinärer Perspektive angezeigt.

Die Analyse der Entwicklungs- und Implementationsprozesse der Basiscurricula zeigt, dass sie sowohl für die Binnen- als auch für die Außenlegitimität der Berufs- und Wirtschaftspädagogik große Orientierungspotenziale bieten. Vor diesem Hintergrund sind weitere Forschungsarbeiten von Interesse, welche die Orientierungsleistungen des Basiscurriculums für die Binnenund Außenlegitimität der Disziplin in den Blick nehmen.

\section{Literatur}

Bank, V. (2009): Berufs- und Wirtschaftspädagogik: Epitaph einer Disziplinlosen. In: bwp@Berufs- und Wirtschaftspädagogik - online 16, 1-22. Online: http://www. bwpat.de/content/uploads/media/bank_bwpat16.pdf (28.11.2016).

4 Die starke Betonung der Forschungsmethoden kann als Versuch interpretiert werden, den Schaden für berufs- und wirtschaftspädagogische Studiengänge abzumildern, der durch die starke staatliche Regulierung entsteht. Diese führt nach Sloane (2010, 4 f.) des Öfteren dazu, dass die Studiengänge von der Scientific Community nicht als forschungsorientiert und somit nicht als gleichwertig zu anderen Studiengängen angesehen werden. 
Beck, K. (2002): Plädoyer für ein Kern-Curriculum zur Ausbildung von Lehrerinnen und Lehrern an berufsbildenden Schulen. Zeitschrift für Berufs- und Wirtschaftspädagogik, 98 (1), 124-130.

Beck, K. (2005): Standards für die Ausbildung von Berufsschullehrern in Europa Professionalisierung im Kompetenzkorsett? Johannes Gutenberg-Universität Mainz. Mainz (Arbeitspapiere WP, 52). Online: http://www.wipaed.uni-mainz.de /ls/ArbeitspapiereWP/gr_Nr.52.pdf (28.11.2016).

Brand, W.; Tramm, T. (2002): Notwendigkeit und Problematik eines Kerncurriculums für die Ausbildung von Berufs- und Wirtschaftspädagogen. In: S. Baabe, E. M. Haarmann \& I. Spiess (Hrsg.): Für das Leben stärken - Zukunft gestalten. Behindertenpädagogische, vorberufliche und berufliche Bildung - Verbindungen schaffen zwischen Gestern, Heute und Morgen, (S. 266-277). Paderborn: Eusl.

Buchmann, U.; Kell, A. (2001): Abschlußbericht zum Projekt Konzepte zur Berufsschullehrerbildung im Auftrag des Bundesministeriums für Bildung und Forschung (Förder-Kennzeichen: K 61.16.00). Siegen.

DGfE (2010): Kerncurriculum Erziehungswissenschaft. Empfehlungen der Deutschen Gesellschaft für Erziehungswissenschaft (DGfE). Opladen, Farmington Hills: Verlag Barbara Budrich (Mitteilungen der DGfE, 21).

HRK (2004): Bologna-Reader I. Texte und Hilfestellungen zur Umsetzung der Ziele des Bologna-Prozesses an deutschen Hochschulen. Bonn: HRK (Beiträge zur Hochschulpolitik, 8). Online: http:/www.hrk.de/fileadmin/redaktion/hrk/02Dokumente/02-10-Publikationsdatenbank/Beitr-2004-08_Bologna-Reader_I.pdf (28.11.2016).

KMK (1999): Rahmenordnung für die Diplomprüfung im Studiengang Wirtschaftspädagogik an Universitäten und gleichgestellten Hochschulen. Online: http://www. kmk.org/fileadmin/veroeffentlichungen_beschluesse/1999/1999_03_ 19-RO-W-Paedagogik-HS.pdf (28.11.2016).

KMK (2002): Künftige Entwicklung der länder- und hochschulübergreifenden Qualitätssicherung in Deutschland. Online: http://www.kmk.org/fileadmin/veroeffentlichungen_beschluesse/2002/2002_03_01-Qualitaetssicherung-laender-hochschuluebergreifend.pdf (28.11.2016).

KMK (2010): Ländergemeinsame Strukturvorgaben für die Akkreditierung von Bachelor- und Masterstudiengängen. Online: http://www.kmk.org/fileadmin/veroeffentlichungen_beschluesse/2003/2003_10_10-Laendergemeinsame-Strukturvorgaben.pdf (28.11.2016).

Lepsius, R. M. (1973): Gesellschaftsanalyse und Sinngebungszwang. In: G. Albrecht, H. Daheim \& F. Sack (Hrsg.): Soziologie: Sprache, Bezug zur Praxis, Verhältnis zu anderen Wissenschaften. René König zum 65. Geburtstag, (S. 105-116). Opladen: Westdeutscher Verlag.

o. V. (1999): Der europäische Hochschulraum. Gemeinsame Erklärung der europäischen Bildungsminister. 19. Juni 1999. Bologna. Online: https://www.hrk.de/file admin/redaktion/hrk/02-Dokumente/02-03-Studium/02-03-01-Studium-Studienreform/Bologna_Dokumente/Bologna_1999.pdf (28.11.2016).

Pätzold, G. (2012): Lehrerbildung für berufsbildende Schulen als herausfordernde Gestaltungsaufgabe. In: M. Becker, G. Spöttl \& T. Vollmer (Hrsg.): Lehrerbildung in Gewerblich-Technischen Fachrichtungen, (S. 11-33). Bielefeld: Bertelsmann (Berufsbildung, Arbeit und Innovation, 37). 
Reinisch, H. (2009): Über Nutzen und Schaden des Philosophierens über das Selbstverständnis der Berufs- und Wirtschaftspädagogik. Anmerkungen aus wissenschaftssoziologisch inspirierter Sicht.bwp@Berufs- und Wirtschaftspädagogikonline 16, 1-18. Online: http:/www.bwpat.de/content/uploads/media/ reinisch_bwpat16.pdf (28.11.2016).

Reuter, L. R. (2007): Zur Akkreditierung von Studiengängen im Rahmen des Bologna-Prozesses am Beispiel erziehungswissenschaftlicher Studienprogramme. Erziehungswissenschaft, 18 (35), 116-125.

Sektion BWP der DGfE (2003): Basiscurriculum für das universitäre Studienfach Berufs- und Wirtschaftspädagogik. Jena. Online: http://www.bwp-dgfe.de/images /Dokumente/Basiscurriculum_BWP_040202.pdf (28.11.2016).

Sektion BWP der DGfE (2014): Basiscurriculum für das universitäre Studienfach Berufs- und Wirtschaftspädagogik im Rahmen berufs- und wirtschaftspädagogischer Studiengänge. Schwäbisch-Gmünd. Online: http://www.bwp-dgfe.de/ images/Dokumente/Basiscurriculum_Berufs-und_Wirtschaftspaedagogik_2014. pdf (28.11.2016).

Sloane, P. F. E. (2003): Bakkalaureaten und Magister für die (berufsbildenden) Schulen - Quo vadis Berufs- und Wirtschaftspädagogik. Zeitschrift für Berufs- und Wirtschaftspädagogik, 99 (4), 481-493.

Sloane, P. F. E. (2010): Avanti dilettanti: die Reform der Lehrerbildung. Zur Einführung des Masters of Education (M.Ed.) und der vielleicht vergeblichen Hoffnung auf Professionalität. Zeitschrift für Berufs- und Wirtschaftspädagogik, 106 (1), 110.

Söll, M. (2016): Die Entwicklung von Studiengängen. Eine Curriculumanalyse am Beispiel der Wirtschaftspädagogik. Detmold: Eusl (Wirtschaftspädagogisches Forum, 55).

Tenorth, H.-E. (2004): Bildungsstandards und Kerncurriculum. Systematischer Kontext, bildungstheoretische Probleme. Zeitschrift für Pädagogik 50 (5), 650-661.

Walm, M.; Wittek, D. (2014): Lehrer_innenbildung in Deutschland im Jahr 2014. Eine phasenübergreifende Dokumentation der Regelungen in den Bundesländern - Eine Expertise im Auftrag der Max-Traeger-Stiftung. GEW Gewerkschaft Erziehung und Wissenschaft. Online: https://www.gew.de/index.php?eID=dump File \&t=f\&f=23490\&token=270cc087a163e0ebb51f76950df2efa377f1d091\&sdown load $=\& n=$ Lehrer-Innenbildung_2014_A4_web.pdf (28.11.2016).

Wilbers, K. (2004): Standards für die Bildung von Lehrkräften. Arbeitsbericht. St. Gallen.

Wildt, J. (2007): Vom Lehren zum Lernen. In: F. Bretschneider \& J. Wildt (Hrsg.): Handbuch Akkreditierung von Studiengängen. Eine Einführung für Hochschule, Politik und Berufspraxis, (S. 44-54). Bielefeld: Bertelsmann (GEW-Materialien aus Hochschule und Forschung, 110).

Wissenschaftsrat (2001): Empfehlungen zur künftigen Struktur der Lehrerbildung. Berlin. Online: http://www.wissenschaftsrat.de/download/archiv/5065-01.pdf (28.11.2016).

Wolter, A.; Kerst, C. (2008): Akkreditierung als Verfahren der Qualitätssicherung von Studiengängen in Deutschland. Eine Policy-orientierte Analyse. In: E. Klieme \& R. Tippelt (Hrsg.): Qualitätssicherung im Bildungswesen, (S. 235-155). Weinheim [u. a.]: Beltz (Zeitschrift für Pädagogik, Beiheft 53). 
Zlatkin-Troitschanskaia, O.; Kuhn, C. (2010): Messung akademisch vermittelter Fertigkeiten und Kenntnisse von Studierenden bzw. Hochschulabsolventen. Analyse zum Forschungsstand. Johannes Gutenberg-Universität Mainz. Mainz (Arbeitspapiere WP, 56). Online: http:/www.wipaed.uni-mainz.de/ls/Arbeitspapiere WP/gr_Nr.56.pdf, (28.11.2016). 


\title{
Kooperatives Lernen im kaufmännischen Unterricht. Eine Netzwerkanalyse zu sozial-konstruktivistischen Lerntheorien
}

\author{
Christoph Helm, Jacqueline Netzthaler und Bettina Kreuzer
}

\section{Einleitung}

Soziale Beziehungen sind sowohl für das Lernen in der Schule als auch für berufliche Tätigkeiten von hoher Bedeutung. Sozial-kognitive Lerntheorien (Vygotsky 1978) postulieren, dass bei leistungsschwächeren Lerner/inne/n insbesondere dann Lernprozesse angestoßen werden, wenn sie Unterstützung von kompetenteren bzw. erfahreneren Peers/Personen erhalten. Diese Unterstützung ermöglicht ihnen das Lösen von Problemen der nächsthöheren Entwicklungszone, die sie alleine nicht erreichen würden. Kooperations- und Teamfähigkeit sind auch von Unternehmen häufig geforderte Skills, um die Anforderungen einer modernen Arbeitsorganisation zu bewältigen (z. B. Euler 2016). So zeigen Trost und Weber $(2012,227)$ in einer Analyse von 707 Stellenanzeigen, dass in drei von vier Anzeigen soziale Kompetenzen und interpersonelle Orientierungen gefordert werden. Vor diesem Hintergrund stellt die Förderung sozialen Lernens und kooperativer Fähigkeiten eine zentrale Aufgabe des (Berufs-)Bildungswesens dar, die u. a. durch die Förderung kooperativen Lernens in den Curricula fest verankert ist (für Deutschland: KMK 2004, für Österreich: BMBF 2014, für die Schweiz: SBFI 2011).

Auch die Bildungsforschung hat sich bereits früh zur Aufgabe gemacht, kooperative Lernprozesse zu untersuchen (bspw. Allport 1924; Watson 1928; Shaw 1932; siehe Gillies \& Ashman 2003 für eine geschichtliche Abhandlung zum kooperativen Lernen). Besonderen Bekanntheitsgrad genießen die einschlägigen (Meta-)Meta-Studien von Johnson, Maruyama, Johnson, Nelson und Skon (1981), Slavin (1995) und Hattie (2009), die versuchen, die umfassende Forschungslage zu Effekten und Gelingensbedingungen kooperativen Lernens zusammenzufassen. Sie berichten für das kooperative Lernen überaus positive Effekte auf die kognitive und non-kognitive Entwicklung der Lernenden. Erklärungen für diese positiven Effekte liefern Theoriearbeiten. Die am häufigsten zur Erklärung kooperativen Lernens herangezogenen Theorien stellen die (neo-)piagetsche, sozio-kognitive Theorie und das (neo)vygotskysche Konzept der Zone der nächsthöheren Entwicklung dar (vgl. bspw. Renkl 1997; Krause 2007). Sie postulieren, dass kooperatives Lernen 
insbesondere dann positive Effekte auf das Schülerlernen entfaltet, wenn jene, die Lernunterstützung (z. B. durch Erklärungen) geben, über einen Kompetenzvorsprung verfügen. Renkl (1997, 29 ff.) argumentiert unter Rückgriff auf die erwähnten Theorien, dass von helfenden Schüler/inne/n (z. B. Tutor/inn/en) erwartet wird, dass sie stärker strukturiertes und organisiertes Wissen besitzen und den Lernstoff besser beherrschen, was ihnen ermöglicht,

- das „Nicht-Verstehen“ anderer diagnostizieren zu können;

- kognitive Konflikte zu induzieren, etwa durch sokratische (Rück-) Fragen, und diese durch (Ko-)Elaboration wieder aufzulösen;

- gute Erklärungen zu geben, d. h. ihr Wissen weitergeben (externalisieren) zu können, sodass der/die Hilfesuchende das Wissen aufnehmen (internalisieren) kann;

- Rückfragen von Hilfesuchenden zu beantworten sowie

- nicht in ihrer Lehrerrolle oder durch Rückfragen überfordert zu werden.

Es ist anzumerken, dass kooperatives Lernen auch zwischen ähnlich kompetenten Lernpartner/inne/n und/oder bidirektional funktionieren kann. Im vorliegenden Beitrag konzentrieren wir uns aber auf die Annahme, dass kooperative Lerneffekte wahrscheinlicher sind, wenn die unterstützende Person ähnlich einer Lehrperson - über einen Kompetenzvorsprung verfügt, gleichzeitig aber durch die Peer-Beziehung das Autoritätsgefälle Lehrer/inSchüler/in wegfällt und so ,die Wahrscheinlichkeit größer [ist], dass jeder frei seine Ansichten äußert und es damit zu sozio-kognitiven Konflikten kommt, welche kognitive Umstrukturierungen initiieren“ (Renkl 1997, 349).

Die Frage, ob Schüler/innen, die andere Schüler/innen beim Lernen unterstützen, im Sinne der angeführten Lerntheorien und ihrer Argumente, auch über höhere fachliche und soziale Fertigkeiten verfügen, ist dagegen noch kaum erforscht. Insgesamt liegen für den berufsbildenden Bereich im deutschsprachigen Raum nur wenige empirische Befunde zum kooperativen Lernen vor. So ist im Themenheft 21 Kooperatives Lernen in der beruflichen Bildung (Euler, Pätzold \& Walzik 2007) der Zeitschrift für Berufs- und Wirtschaftspädagogik (ZBW) lediglich ein empirischer Beitrag (Gräsel, Stark, Sparka \& Herzmann 2007) abgedruckt. In der aktuellen Ausgabe der ZBW (Jahrgang 112, Heft 4) hingegen finden sich mehrere Beiträge zur Frage der Operationalisierung sozialer Kompetenzen. Den wenigen vorliegenden Studien ist gemein, dass sie meist mit Selbsteinschätzungsskalen das Phänomen des kooperativen Lernens (z. B. Euler, Jüttler, Raatz \& Schumann 2016) oder die soziale Kompetenz (z. B. Tschöpe \& Monnier 2016) untersuchen (mit Ausnahmen wie Studien von Güzel, Nickolaus, Zinn, Würmlin \& Sari 2016, in der darüber hinaus Dilemmasituationen eingesetzt werden). Die soziale Netzwerkanalyse (SNA) als Methode zur Erforschung sozialen Lernens wur- 
de dagegen unseres Wissens bisher nicht eingesetzt. Dies ist insofern unerfreulich, da die SNA besonderes Potential birgt, das offenbar für die Erklärung von schulischem Lernen noch nicht genutzt wurde: Es wird kritisiert, dass in der vorherrschenden Untersuchungspraxis relationale Beziehungen zwischen Lernenden immer noch außer Acht gelassen und die Attribute einzelner Untersuchungseinheiten (z. B. Intelligenz, Geschlecht) fokussiert werden (Carolan 2013, 11; Zander Kolleck \& Hannover 2014, 3). „So ist davon auszugehen, dass die Berücksichtigung sozialer Beziehungen und Einflussprozesse innerhalb von Klassenräumen eine wertvolle, vielleicht sogar notwendige Erklärungsperspektive für die Lehr- und Lernforschung eröffnet" (Wölfer \& Cortina 2014, 192). Im vorliegenden Beitrag stellen wir mit unseren Analysen beispielhaft dar, wie dieses bisher wenig genutzte Potential der SNA für die Analyse kooperativen Lernens genützt werden kann. Das Ziel des Beitrags liegt allerdings nicht in der Untersuchung der Effekte des kooperativen Lernens, sondern darin, zu zeigen, wie kooperatives Lernen in Klassensettings funktioniert. Dazu überprüfen wir die angeführten sozial-konstruktivistischen Annahmen. Konkret untersuchen wir auf Basis von Daten aus 25 Klassen berufsbildender mittlerer und höherer Schulen (BMHS) in Österreich die Annahmen, dass (a) leistungsfähige Schüler/innen und (b) Schüler/innen, die sich selbst als sozial kompetent einschätzen, häufiger leistungsschwächeren Schüler/inne/n helfen als andere Schüler/innen.

\section{Wie funktioniert kooperatives Lernen?}

Im Folgenden werden zentrale Argumente unterschiedlicher theoretischer Zugänge - die sich aufgrund gemeinsamer Wurzeln stark überlappen -vorgestellt. Allen gemein ist, dass sie versuchen, die Funktionsweise des kooperativen Lernens zu erläutern. Gleichzeitig sollen damit auch die Vorzüge kooperativen Lernens gegenüber alleingängigem Lernen verdeutlicht werden.

\subsection{Argumente aus Sicht der kognitiven Elaborationstheorie}

Erweiterung bestehender Schemata: Kognitive Elaboration meint die Vernetzung von neuen Konzepten, Schemata oder Unterrichtsinhalten mit bereits vorhandenem Wissen (Wellenreuther 2010, 390). Diese aktive Verknüpfung soll zu einem besseren Verständnis führen. Kooperatives Lernen schafft durch den sozialen Austausch günstige Rahmenbedingungen für diese Form des Lernens (Konrad 2014, 63; Wellenreuther 2010). Bei Partner- oder Gruppenarbeiten - so die Idee - unterstützen sich die Lernenden gegenseitig beim Aneignen neuer Inhalte. Dabei sind die Schüler/innen im Gegensatz zum 
alleingängigen Lernen gezwungen ihre Gedankengänge und Überlegungen wiederzugeben, um die Lernpartner/innen daran teilhaben zu lassen. Genau diese Artikulation ihrer Gedanken führt dazu, dass eine Verbindung zum bereits vorhandenen Wissen aufgebaut wird. Laut Renkl (1997, 42) wird durch das selbstständige und gegenseitige Erklären des Lehrstoffes das Verständnis über Lerninhalte vertieft, u. a. indem die Verknüpfungen zwischen den alten und neuen Lerninhalten ausgebaut werden.

Erkennen von Wissenslücken: Nach Chi, Bassok, Lewis, Reimann und Glaser (1989) funktioniert Lernen in kooperativen Settings nicht nur, wie eben beschrieben, über die Elaboration neuer Wissensstrukturen, sondern auch über das Herauskristallisieren von Wissenslücken. Erfolgt das Erlernen der Stoffinhalte durch kooperatives Lernen, wird den Lernenden durch die Artikulation der Inhalte bewusst, was sie noch nicht verstanden haben, sodass sie diese Lücken durch gegenseitige Unterstützung schließen können.

\subsection{Argumente aus kognitiver Entwicklungsperspektive}

Lernen von Kompetenteren: Laut Slavin (1993, 536 f.) ist Schülerlernen in heterogenen Gruppen wahrscheinlicher, da sich die Schüler/innen gegenseitig helfen können. Vor allem leistungsschwächere können von leistungsstärkeren Schüler/inne/n lernen/profitieren. Die Zusammenarbeit mit kompetenteren Gleichaltrigen unterstützt damit den Lernprozess (Slavin 1993, 546).

Lernen von Gleichaltrigen: Auch für Wellenreuther $(2010,390)$ ist kooperatives Lernen für die Entwicklung der Schüler/innen von großer Bedeutung. Die Kooperation unter Gleichaltrigen sieht er als entwicklungsförderlich an, weil die Lernenden innerhalb derselben Entwicklungsstufen miteinander adäquat kommunizieren können und somit gemeinsam nächsthöhere Entwicklungszonen erreichen können.

Überwindung egozentrischer Perspektiven: Für Slavin (1993, 536 f.) ist weiterhin die Überwindung der egozentrischen Perspektive ein Vorteil kooperativen Lernens. Durch das Interagieren der Schüler/innen werden die Grenzen des eigenen Standpunkts bzw. der eigenen Perspektive überwunden. Die Schüler/innen profitieren dabei, indem sie bei schwierigen und kontrovers zu diskutierenden Aufgaben die Perspektive der anderen erkennen, übernehmen und evaluieren können und nicht nur ihre eigene Perspektive bewerten (vgl. Maier 2012, 227).

\subsection{Argumente aus Sicht des sozialen Konstruktivismus}

Proximal Zone of Development: Im sozial-konstruktivistischen Ansatz spielt das Konzept der nächsthöheren Entwicklungszone eine zentrale Rolle. Die Definition dieser Zone nach Vygotsky $(1978,86)$ lautet wie folgt: „It is the 
distance between the actual developmental level as determined by independent problem solving and the level of potential development as determined through problem solving under adult guidance or in collaboration with more capable peers."

Für effizientes Lernen ist deshalb soziales Lernen bedeutend, das durch gegenseitige Unterstützung das Agieren in der nächsthöheren Entwicklungsstufe ermöglicht (Renkl 1997, 36 f.). Die nächsthöhere Entwicklungszone definiert Problemlösungsprozesse, welche die Lernenden zuerst nur durch soziale Unterstützung erreichen können und welche Fähigkeiten beinhalten, die zwar noch nicht herangereift sind, sich jedoch im Reifungsprozess befinden. Durch die Internalisierung dieser Problemlösungsprozesse, die auf sozialer Ebene gemeinsam durchlaufen werden, wird die nächsthöhere Entwicklungsstufe zum aktuellen Entwicklungsstand: Die Lernenden sind in der Lage die Anforderungen, die sie zuerst nur durch soziale Hilfestellung gemeinsam bewältigen konnten, ohne fremde Hilfe alleine zu meistern. Die sozialen Prozesse stellen damit den zentralen Lernmechanismus dar. Die Kooperation mit Kompetenteren ist jedoch nicht immer notwendige Voraussetzung, um sich in die Zone der proximalen Entwicklung bewegen zu können. Peers, die einigermaßen gleich kompetent sind, können sich durch Zusammenarbeit gegenseitig unterstützen, um so ein höheres Entwicklungsniveau zu erreichen, als es den Lernenden ohne Kooperation möglich wäre. In der vygotskyschen Tradition wird allerdings meist angenommen, dass sich jene, die vom kooperativen Lernen profitieren, auf einem niedrigeren Kompetenzniveau als ihre Lernpartner/innen befinden.

Cognitive Apprenticeship: In der Theorie der kognitiven Lehre nach Collins, Brown und Newman (1989) spielt das kognitive Modellieren eine wichtige Rolle. Kognitives Modellieren bedeutet, dass der/die Lehrende die Strategie zur Lösung eines Problems demonstriert und dabei die entsprechenden Problemlösungsprozesse verbalisiert. Die weniger erfahrene Person kann sich durch die Zusammenarbeit mit der erfahreneren Person, also dem/der Lehrenden, die kognitiven Fähigkeiten oder Kompetenzen durch effektive kognitive Verarbeitung aneignen. Dabei führt die weniger erfahrene Person die Aufgabenlösung schrittweise immer selbständiger durch. Der kognitive Verarbeitungsprozess einer Person, geht von der sozialen Ebene (gemeinsame Problemlösung) auf die individuelle Ebene (selbständige Problemlösung) über, sobald eine weniger erfahrene Person die kognitiven Funktionen in sein/ihr Repertoire aufnimmt (Seidel 2014, 281).

\subsection{Voraussetzungen für erfolgreiches kooperatives Lernen}

Johnson und Johnson $(2008,17)$ definieren Kriterien für effektives kooperatives Lernen: (1) Positive Interdependenz, (2) Individuelle Verantwortlichkeit 
und Gruppenbewertung, (3) Soziale Fertigkeiten und Arbeitstechniken, (4) förderliche Face-To-Face-Interaktionen und (5) Reflexion über Gruppenprozesse. Für die vorliegende Untersuchung sind insbesondere die Erfolgskriterien drei und vier zentral, da diese nach Euler (2016, 517) nicht nur Ziel, sondern auch Voraussetzung für kooperatives Lernen sind. Damit kooperatives Lernen überhaupt stattfinden kann, ist ein gewisses Maß an interpersonalen Kompetenzen notwendig. Laut Johnson und Johnson (1987) sind spezielle Fähigkeiten wichtig, damit ein Team gut funktionieren kann. Das Bemühen der Mitglieder ist für gute Teamarbeit alleine nicht ausreichend, es bedarf auch einer guten Kommunikation untereinander, sowie der Fähigkeit, Vertrauen aufzubauen und ein gutes Klima innerhalb der Gruppe zu schaffen.

Neben einer gewissen sozialen Kompetenz ist nach Renkl (1997) - wie bereits eingangs ausführlich beschrieben - von Vorteil, wenn helfende Schüler/innen, die sich gewissermaßen in eine Tutor- bzw. Lehrerrolle begeben, über einen Kompetenzvorsprung (elaborierteres Wissen) verfügen, der ihnen ermöglicht, zentrale Aspekte eines guten Lehrprozesses (z. B. verständliche Erklärungen geben, Wissenslücken diagnostizieren, Fragen wecken und beantworten, ...) zu übernehmen.

\subsection{Forschungsbefunde zum kooperativen Lernen}

Die Recherche in einschlägigen Publikationsorganen der Berufs- und Wirtschaftspädagogik (BWP) zeigt, dass kooperative Lernprozesse in beruflichen Teil- und Vollzeitschulen sowie bei Auszubildenden im Betrieb noch wenig erforscht sind (vgl. Euler 2016) und insbesondere keine SNA-Studien vorliegen. Daher berichten wir themenverwandte Studien, um zu zeigen, dass kooperatives Lernen in der BWP durchaus bereits erforscht wurde.

Vor etwas mehr als zehn Jahren beschäftigten sich verschiedene Forschergruppen (z. B. Euler, Pätzold \& Lang 2005; Nickolaus, Heinzmann \& Knöll 2005; Sembill \& Seifried 2006) mit der Konzeption und Erforschung methodischer Grundentscheidungen (bspw. SoLe, SKOLA). Im Rahmen dieser Entwicklung wurde auch das kooperative Lernen thematisiert; allerdings eher am Rande, sodass keine empirischen Analysen etwa zur didaktischen Umsetzung oder zu Effekten kooperativen Lernens vorliegen. In der allgemeinen Bildungsforschung haben in den 1990er Jahren die auch heute noch oft zitierten Meta-Analysen für Aufsehen gesorgt. Johnson und Johnson (1999) zeigen in ihrer Meta-Analyse, dass in kooperativen Lerngruppen größere Leistung und höhere Produktivität erreicht werden können als beim Lernen in kompetitiven und individuellen Settings. Weiterhin wurde festgestellt, dass kooperatives Lernen den Wissenstransfer begünstigt und Transferprozesse beschleunigt. Auch Slavins Meta-Analyse (1995) kommt zu einem positiven Bild über die Effekte kooperativen Lernens: „Die Ergebnisse 
belegen eine signifikante Überlegenheit kooperativer Lernformen gegenüber traditionellen bzw. kompetitiv angelegten Unterrichtsmethoden. Interessant dabei ist, dass jene kooperativen Lernformen die höchsten Effektstärken zeigen, die nicht nur Gruppenziele [...], sondern auch individuelle Verantwortlichkeit für das Gruppenergebnis (das durchschnittliche Lernergebnis aller Gruppenmitglieder wird bewertet, d. h. die Gruppe muss dafür sorgen, dass alle Mitglieder den Lernstoff gelernt haben) fördern“ (Gruehn 2000, 49).

Die Ergebniszusammenstellung von 180 Studien der Forschergruppe um Johnson und Johnson (1999, 72 f.) zeigt, dass sich durch kooperatives Lernen im Unterricht die Beziehungen der Schüler/innen untereinander verbessern. Die positive Beziehung zwischen den Lernenden hat wiederum höhere Produktivität, höhere persönliche Verbundenheit mit der Gruppe, höhere Zielerreichungsmotivation und Verantwortungsübernahme zur Folge und wirkt sich somit vorteilhaft auf die Gruppenleistung aus. Drössler $(2010,68)$ verweist zudem darauf, dass in kooperativen Lerngruppen isolierte bzw. introvertierte Schüler/innen besser integriert wurden. Im Unterschied zu individuellen und konkurrierenden Lernmethoden sind Schüler/innen beim kooperativen Lernen davon überzeugt, von anderen akzeptiert und unterstützt $\mathrm{zu}$ werden (Johnson \& Johnson 1999, 73).

\section{Methode}

\subsection{Hypothesen}

Die sozial-konstruktivistische Lerntheorie postuliert, dass kooperatives Lernen insbesondere dann stattfindet, wenn kompetentere mit leistungsschwächeren Schüler/inne/n interagieren bzw. diese im Lernprozess unterstützen, sodass diese höhere Entwicklungsniveaus erreichen können. Die potentiellen Profiteure des kooperativen Lernens sind demnach die weniger kompetenten Schüler/innen. Aus dieser Überlegung leiten wir Hypothese 1 ab: Beim kooperativen Lernen werden leistungsschwache Schüler/innen eher von leistungsstarken als von leistungsschwachen Mitschüler/inne/n unterstützt.

Aus den Forschungsbefunden zu den Erfolgskriterien kooperativen Lernens leiten wir Hypothese $2 \mathrm{ab}$ : Beim kooperativen Lernen werden leistungsschwache Schüler/innen eher von Mitschüler/inne/n unterstützt, die sich als sozial kompetent einschätzen.

Die Untersuchung der Hypothesen soll Aufschluss darüber geben, ob kooperatives Lernen im Unterricht durch Beziehungen charakterisiert ist, die den hier postulierten sozial-konstruktivistischen Annahmen entsprechen. 


\subsection{Beschreibung der Stichprobe}

Zur Hypothesenprüfung wurden Daten von 539 Schüler/inne/n (Alter: $M=$ $16,33, S D=0,74,75 \%$ weiblich) aus 25 Klassen am Ende der 10. Schulstufe der BMHS in Österreich analysiert. Da fehlende Schülerangaben bei sozialen Netzwerkanalysen dazu führen, dass sich die auf Basis der Ergebnisse getätigten Aussagen lediglich auf die beobachteten Teile des untersuchten, „wahren" Netzwerkes beziehen, können diese verzerrt sein. Dies gilt besonders dann, wenn die fehlenden Werte nicht zufällig verteilt sind. Aus diesem Grund führen wir alle Analysen auf Basis a) aller Klassen und b) nur jener Klassen, die weniger als $20 \%$ fehlende Werte aufweisen (Skvoretz 2015), durch, um so die Stabilität der Befunde zu prüfen.

\subsection{Konstruktoperationalisierung}

\subsubsection{Soziale Kompetenz}

Für die Erhebung der sozialen Kompetenz wurden der Interpersonal Competence Questionnaire (ICQ; Riemann \& Allgöwer 1993) und die Skala Soziale Unterstützung im Unterricht (SUU), die sich aus in unterschiedlichen Studien (Kunter et al. 2002; Quellenberg 2009; Eberle et al. 2009) erprobten Items zusammensetzt, herangezogen. Der ICQ erfasst mit je acht Items fünf umfassende, theoretisch bedeutsame und voneinander abgrenzbare Aufgabenbereiche bzw. Dimensionen der Sozialkompetenz (Riemann \& Allgöwer 1993, 154, siehe Tab. 1). Die fünfstufigen Antwortkategorien der Items reichen von -2 ,Dieses Verhalten auszuführen, gelingt mir gewöhnlich schlecht. Ich fühle mich unwohl in einer solchen Situation.“ bis +2 ,Dieses Verhalten auszuführen, gelingt mir gewöhnlich gut. Ich fühle mich wohl in einer solchen Situation." Die Teilung der Stichprobe in eine sozial kompetente und eine weniger sozial kompetente Schülergruppe erfolgte in Variante 1 am Mittelwert der Gesamtskala des ICQs $(M=3,57)$ und in Variante 2 am 70. Perzentil ${ }^{1}(3,85)$. Die Items der SUU-Skala sind im Gegensatz zu jenen des ICQs auf den Schulalltag bezogen. Das Antwortformat dieser Items ist ebenfalls fünfstufig ( $1=$,nie/stimmt gar nicht“ bis $5=$ „,fast täglich/stimmt genau“). Die Teilung der Stichprobe erfolgte auch hier am Mittelwert der Gesamtskala $(M=3,25)$ sowie am 70. Perzentil $(3,50)$. Tabelle 1 enthält Beispielitems und die Reliabilitäten der verwendeten Skalen.

1 Die Wahl des 70. Perzentils stellt eine übliche Vorgehensweise dar, um Extremgruppen zu identifizieren. Dies gilt auch für die Leistungsfähigkeit. 
Tab. 1: Operationalisierung der Sozialkompetenz und des Sozialverhaltens im Unterricht

\begin{tabular}{|c|c|}
\hline Konstruktbeschreibung & Beispielitem \\
\hline \multicolumn{2}{|c|}{ ICQ: Initiierung von Interaktionen und Beziehungen (.81) } \\
\hline $\begin{array}{l}\text { Die acht Items messen den Umgang } \\
\text { und die Interaktion mit neuen Be- } \\
\text { kanntschaften. }\end{array}$ & $\begin{array}{l}\text { Mit einer neuen Bekanntschaft, die du } \\
\text { näher kennenlernen möchtest, Gespräche } \\
\text { führen. }\end{array}$ \\
\hline \multicolumn{2}{|l|}{ ICQ: Durchsetzungsfähigkeit (.80) } \\
\hline \multirow{3}{*}{\multicolumn{2}{|c|}{$\begin{array}{ll}\text { Diese Dimension fragt mit acht Items } & \text { Auf dein } \\
\text { nach der Durchsetzungsfähigkeit der } & \text { Freund } \\
\text { Proband/inn/en, sowie deren Kritik- } & \text { dir gege } \\
\text { verhalten. } & \end{array}$}} \\
\hline & \\
\hline & \\
\hline \multicolumn{2}{|c|}{ ICQ: Preisgabe persönlicher Informationen (.74) } \\
\hline $\begin{array}{l}\text { Es wird mit acht Items gemessen, ob } \\
\text { die Proband/inn/en ihren Bekannt- } \\
\text { schaften schnell Vertrauen schenken } \\
\text { und somit persönliche Informationen } \\
\text { preisgeben. }\end{array}$ & $\begin{array}{l}\text { Ein Gespräch mit einer neuen Bekannt- } \\
\text { schaft von Oberflächlichkeiten weglen- } \\
\text { ken, um sich gegenseitig wirklich ken- } \\
\text { nenzulernen. }\end{array}$ \\
\hline \multicolumn{2}{|c|}{ ICQ: Emotionale Unterstützung anderer (.87) } \\
\hline $\begin{array}{l}\text { Acht Items erfassen die Empathie } \\
\text { sowie die Fähigkeit der Pro- } \\
\text { band/inn/en, ihre Mitmenschen emoti- } \\
\text { onal zu unterstützen. }\end{array}$ & $\begin{array}{l}\text { Einem/r guten Freund/in helfen, seine/ } \\
\text { ihre Gedanken und Gefühle bezüglich } \\
\text { einer wichtigen Lebensentscheidung zu } \\
\text { ordnen, ... }\end{array}$ \\
\hline \multicolumn{2}{|l|}{ ICQ: Regelung von Konflikten (.77) } \\
\hline $\mathrm{Zu}$ dieser Dimension gehören acht & Während eines Konflikts mit einem/r \\
\hline Items, die nach dem Umgang und der & guten Freund/in, nicht versuchen die \\
\hline $\begin{array}{l}\text { Regelung bei einem entstandenen } \\
\text { Konflikt fragen. }\end{array}$ & $\begin{array}{l}\text { Gedanken des anderen zu erahnen, son- } \\
\text { dern seine/ihre Beschwerde wirklich } \\
\text { anhören. }\end{array}$ \\
\hline
\end{tabular}

SUU: Soziales Engagement/soziale Verantwortungsübernahme (.73)

Fünf Items erfassen das soziale Enga- Ich habe mich selbst gegen Störungen im gement der Schüler/innen im Unter- Unterricht eingesetzt. richt allgemein (Quellenberg 2009).

SUU: Unterstützung anderer im Unterricht (.78)

Drei Items messen die Unterstützung Wie oft versuchst du das, was du gelernt der Mitschüler/innen bei unterrichts- hast, an deine Mitschüler/innen weiterbezogenen Aktivitäten durch den/die zugeben?

Proband/in (Kunter et al. 2002).

SUU: Fähigkeit der Schüler/innen zur Perspektivenübernahme (.81)

Vier Items messen die Fähigkeit der Bei Meinungsverschiedenheiten versuche

Schüler/innen zur Perspektivenüber- $\quad$ ich, die Sache aus Sicht aller Beteiligten

nahme (Quellenberg 2009).

zu betrachten, bevor ich mich entscheide. 
Tab. 1: Operationalisierung der Sozialkompetenz und des Sozialverhaltens im Unterricht (Fortsetzung)

SUU: Konfliktfähigkeit (.83)

Sechs Items messen die Konfliktfä-

higkeit (Reaktion und Verhaltenswei-

sen bei einem Konflikt) (Eberle et al.

2009).
Ich akzeptiere berechtigte Argumente der anderen, auch wenn sie meinen Argumenten widersprechen.

Quelle: Eigene Darstellung

\subsubsection{Leistungsfähigkeit}

Die Leistungsfähigkeit der Schüler/innen wurde einerseits durch selbstberichtete Jahresnoten der Fächer Rechnungswesen, Betriebswirtschaft und Deutsch (Sehr gut, Gut, Befriedigend, Genügend, Nicht genügend) und andererseits durch einen standardisierten Leistungstest (WBB, Helm 2016) am Ende der 10. Schulstufe erfasst. Im Rahmen der Testung mussten die Schüler/innen 34 Aufgaben bearbeiten, die u. a. die Verbuchung von laufenden Geschäftsfällen erforderten:

Ein Beispielitem: Du kaufst Handelswaren im Wert von EUR 14,500.00+ $20 \%$ Umsatzsteuer auf Ziel (3300) für dein Unternehmen. Verbuche den Geschäftsfall und schätze die Auswirkung auf den Gewinn ein!

Die Testitems wurden aus dem Kompetenzmodell der Arbeitsgruppe für die österreichischen Berufsbildungsstandards abgeleitet (http://www.bildungsstandards.berufsbildendeschulen.at). Dieses zweidimensionale Modell verortet Rechnungswesen-Aufgaben in einer Inhalts- (laufende Geschäftsfälle Kostenrechnung) und einer Handlungsdimension (wiederholen - entwickeln). Darüber hinaus decken die Testitems die Kerninhalte des kompetenzorientierten Lehrplans der kaufmännischen BMHS ab (BMBF 2014). Um die Kompetenzwerte der Schüler/innen vor dem Hintergrund der Item Response Theory zu erhalten, wurden (1) die Schülerantworten mit 1 (richtig) und 0 (falsch) kodiert und auf Basis des Rasch-Modells die latenten Schülerfähigkeiten geschätzt. Die Modellberechnungen weisen sowohl auf Item- als auch Testebene zufriedenstellende EAP-/WLE-Reliabilitätswerte auf (zwischen .78 und .89; siehe Helm 2016 für die Rasch-Modellgeltungskontrollen). Die Teilung der Stichprobe in eine leistungsfähige und eine weniger leistungsfähige Schülergruppe erfolgte am Mittelwert (Variante 1) der Noten $(M=2,74)$ bzw. der Kompetenzwerte $(M=0,43)$. Zusätzlich (Variante 2$)$ erfolgte die Gruppenteilung am 70. Perzentil der Kompetenzwerte $(0,74)$. 


\subsubsection{Erhebung der helfenden Beziehung zwischen den Schüler/inne/n}

Die Lernunterstützung zwischen den Schüler/inne/n wurde auf Basis sozialer Netzwerkdaten erfasst, die durch Vorlage der folgenden Frage (SNA-Frage) erhoben wurden: „Kreuze an wie oft du deinem/deiner Klassenkolleg/in/en weiterhilfst, wenn er/sie im Unterricht etwas nicht versteht". Die Schüler/inne/n mussten für jede/n Klassenkollegen/in angeben, wie oft sie ihm/ihr Unterstützung anbieten: keine Hilfe, nie oder fast nie, 1-2mal pro Monat, Imal pro Woche, mehrmals pro Woche, (fast) in jeder Stunde bzw. bei jeder Gelegenheit. Jene, die ,keine Hilfe“ oder „,nie oder fast nie“ wählten, wurden als ,nicht unterstützend“ (0) klassifiziert, alle anderen als ,unterstützend“ (1) (siehe Tabelle 2).

\subsubsection{Operationalisierung der Netzwerkdaten}

Die erhobenen Schülerdaten wurden für die folgenden Analysen in drei Matrizen übertragen. Matrix 1 ,,Unterstützung “ wurde direkt aus der SNA-Frage abgeleitet. Tabelle 2 enthält zu Illustrationszwecken Netzwerkdaten einer fiktiven Klasse.

Tab. 2: Matrix 1 ,Unterstützung“

\begin{tabular}{ccccc}
\hline \multicolumn{5}{c}{ Netzwerkdaten: Unterstützung } \\
\hline \multirow{5}{*}{ Lisa } & Lisa & Thomas & Julia & Andreas \\
Thomas & - & 0 & 0 & 0 \\
Julia & 1 & - & 1 & 0 \\
Andreas & 1 & 0 & - & 1 \\
Anmerkung: & $0=$ unterstützt nicht beim Lernen, 1 = unterstützt mindestens einmal \\
& beim Lernen &
\end{tabular}

Quelle: Eigene Darstellung

Matrix 2 „,Unterstützung durch leistungsfähige Schüler/innen“ wurde durch Kodierung der Beziehung zwischen zwei Schüler/inne/n mit 1 oder 0 entsprechend folgendem Schema erstellt: Die Beziehung wurde mit 1 kodiert, wenn

a. eine unterstützende Beziehung (Tabelle 2) vorliegt UND

b. der/die Unterstützer/in leistungsfähig (i.S. des Leistungstests) ist UND

c. der/die Unterstützte leistungsschwach (im Sinne des Leistungstests) ist.

In allen anderen Fällen wurde die Beziehung mit 0 kodiert. Tabelle 3 enthält zu Illustrationszwecken Netzwerkdaten einer fiktiven Klasse. 
Tab. 3: Matrix 2 ,Unterstützung durch leistungsfähige Schüler/innen“

\begin{tabular}{ccccc}
\hline \multicolumn{5}{c}{ Netzwerkdaten: Leistung } \\
\hline Lisa & Lisa & Thomas & Julia & Andreas \\
Thomas & - & 0 & 1 & 0 \\
Julia & 0 & - & 1 & 0 \\
Andreas & 0 & 0 & - & 0 \\
\hline Anmerkung: & 1, wenn Unterstützer/in = leistungsfähig UND Unterstützte/r $=$ \\
& leistungsschwach; 0 bei alle anderen Konstellationen
\end{tabular}

Quelle: Eigene Darstellung

Matrix 3 „Unterstützung durch sozial kompetente Schüler/innen“ wurde durch Kodierung der Beziehung zwischen zwei Schüler/inne/n mit 1 oder 0 entsprechend folgendem Schema erstellt: Die Beziehung wurde mit 1 kodiert, wenn

a. eine unterstützende Beziehung (Tabelle 2) vorliegt UND

b. der/die Unterstützer/in sich als sozial kompetent (im Sinne der ICQ- bzw. SUU-Skala) einschätzt UND

c. der/die Unterstützte leistungsschwach (im Sinne des Leistungstests) ist.

In allen anderen Fällen wurde die Beziehung mit 0 kodiert. Tabelle 4 enthält zu Illustrationszwecken Netzwerkdaten einer fiktiven Klasse.

Tab. 4: Matrix 3 „Unterstützung durch sozial kompetente Schüler/innen“

\begin{tabular}{ccccc}
\hline \multicolumn{5}{c}{ Netzwerkdaten: Soziale Kompetenz } \\
\hline Lisa & Lisa & Thomas & Julia & Andreas \\
Thomas & - & 0 & 1 & 0 \\
Julia & 0 & - & 0 & 0 \\
Andreas & 0 & 0 & - & 0 \\
\hline
\end{tabular}

Anmerkung: 1, wenn Unterstützer/in = sozial kompetent UND Unterstützte/ $\mathrm{r}=$ leistungsschwach; 0 bei alle anderen Konstellationen

Quelle: Eigene Darstellung

Matrix 2 und 3 wurden sowohl für Variante 1 (Mittelwertsplit) als auch Variante 2 (70. Perzentilsplit) erstellt. 


\subsection{Auswertungsstrategie}

Zur Prüfung der Hypothesen wurden Korrelationen von Matrizen, die soziale Netzwerkdaten enthalten (siehe unten), in R (R Core Team 2014) mit dem Paket „sna“" (Butts 2010) durchgeführt, wobei die Signifikanzberechnungen via Monte Carlo Simulationen (siehe „cugtest“, Butts 2010) erfolgten. Für jede der 25 untersuchten Klassen wurde Matrix 1 „Unterstützung“ mit

1. Matrix 2 (WBB, Mittelwertsplit),

2. Matrix 2 (WBB, 70. Perzentilsplit),

3. Matrix 2 (Noten, Mittelwertsplit),

4. Matrix 3 (ICQ, Mittelwertsplit),

5. Matrix 3 (ICQ, 70. Perzentilsplit),

6. Matrix 3 (SUU-Skala, Mittelwertsplit) und

7. Matrix 3 (SUU-Skala, 70. Perzentilsplit) korreliert.

Die Korrelationswerte wurden über die 25 Klassen gemittelt. Im nachfolgenden Ergebnisteil werden neben dem Mittelwert der 25 Korrelationen auch der Median der Korrelationen sowie die kleinste und größte Korrelation und die Anzahl der Klassen, deren Korrelation nicht signifikant ist, angegeben.

\section{Ergebnisse}

Hypothese 1: Leistungsschwache Schüler/innen werden signifikant häufiger von leistungsstarken als von-schwachen Mitschüler/inne/n unterstützt.

Variante 1 - Mittelwertsplit: Der in Hypothese 1 postulierte Zusammenhang kann sowohl bei Operationalisierung mittels standardisiertem Test als auch mittels Schulnoten bestätigt werden. Im ersten Fall beträgt die Korrelation im Mittel aller 25 Klassen $r=.249$ (Median: $r=.238$, min: .044, max: .504). In fünf Klassen ist die Korrelation nicht signifikant $(p<.05)$. Werden die Jahresschulnoten als Indikator herangezogen, so ergibt sich ein etwas niedriger durchschnittlicher Korrelationskoeffizient von $r=.179$ (Median: .134 [.043-.570], n.s. = 5 Klassen). Unter Ausschluss jener Klassen mit mehr als $20 \%$ fehlenden Schülerangaben ändern sich die Werte kaum: $r=$ .272 (Median: .269, [.058-.503], n.s. = 2) bzw. $r=.171$ (Median: .134 [.050467], n.s. $=2$ Klassen).

Variante 2 - 70. Perzentilsplit: Auch diese Variante führt zur Bestätigung der Hypothese 1: Im Mittel liegt die Korrelation zwischen den Matrizen 1 und 2 der 25 Klassen in Höhe von $r=.176$ (Median: .117 [.046-.692], n.s. $=5$ Klassen), wenn die Leistungsfähigkeit mit dem WBB erfasst wird. Unter Ausschluss jener Klassen mit mehr als $20 \%$ fehlenden Schülerangaben än- 
dern sich die Werte kaum: $r=.159$ (Median: .117 [.055-.386], n.s. = 2 Klassen).

Hypothese 2: Leistungsschwache Schüler/innen werden signifikant häufiger von Mitschüler/inne/n unterstützt, die sich als sozial kompetent einschätzen. Variante 1 - Mittelwertsplit: Der in Hypothese 2 postulierte $\mathrm{Zu}-$ sammenhang lässt sich mit den Daten bestätigen. Sowohl die Operationalisierung sozialer Kompetenz mittels ICQ als auch mittels SUU-Skala führen im Mittel aller 25 Klassen zu einer Korrelation von $r=.271$ (Median: .254 [.026-.567], n.s. $=3$ ) bzw. $r=.264$ (Median: .268 [.039-.707], n.s. $=4$ Klassen). Unter Ausschluss jener Klassen mit mehr als $20 \%$ fehlenden Schülerangaben steigen die Werte minimal an: $r=.299$ (Median: .306 [.081-.567], n.s. $=1$ Klasse) bzw. $r=.293$ (Median: .303 [.066-.707], n.s. $=3$ Klassen).

Variante 2 - 70. Perzentilsplit: Auch Variante 2 führt zur Bestätigung der Hypothese 2: Im Mittel liegt die Korrelation zwischen den Matrizen 1 und 3 der 25 Klassen bei $r=.284$ (Median: .350 [.044-.454], n.s. $=5$ Klassen), wenn die soziale Kompetenz mit dem ICQ erfasst wird. Bei Messung mittels SUU-Skala liegt das mittlere $r$ bei .294 (Median: .380, [.027-.570], n.s. $=5$ ). Unter Ausschluss jener Klassen mit mehr als $20 \%$ fehlenden Schülerangaben steigen die Werte leicht an: $r=.333$ (Median: .383, [.049-.454], n.s. $=3$ Klassen) bzw. $r=.334$ (Median: .385 [.049-.454], n.s. $=3$ Klassen).

\section{Diskussion}

Ziel der vorgelegten Studie ist die Untersuchung sozialer Netzwerke in 25 Klassen kaufmännischer Schulen, um folgende Frage vor dem Hintergrund sozial-konstruktivistischer Annahmen kooperativen Lernens zu beantworten: Werden Lernunterstützungen häufiger von leistungsstarken bzw. sozial kompetenteren Schüler/inne/n für leistungsschwächere angeboten? Die Ergebnisse zeigen, dass Hypothese 1 Leistungsschwache Schüler/innen werden signifikant häufiger von leistungsstarken als von -schwachen Mitschüler/inne/n unterstützt haltbar ist, und zwar unabhängig von der Operationalisierung der Leistungsfähigkeit. Auch Hypothese 2, die davon ausgeht, dass Schüler/innen, die sich selbst als sozial kompetent einschätzen, häufiger leistungsschwächeren Schüler/inne/n helfen, kann unabhängig von der Operationalisierung der sozialen Kompetenz bestätigt werden.

Diese Resultate sind vor dem Hintergrund der dünnen Befundlage zu Merkmalen von Lernpartner/innen und Lerngruppen relevant, da sie Hinweise zum effizienten Peer-Lernen liefern. Laut Johnson und Johnson (1999) kann durch kooperatives Lernen eine höhere Leistungsfähigkeit erreicht werden als bei individuellen oder konkurrierenden Lernformen. Dieser Vorteil zeigt sich gemäß sozial-kognitiven Lerntheorien vor allem dann, wenn leis- 
tungsschwächere Lernende von der Zusammenarbeit mit leistungsfähigeren Personen profitieren. Die Ergebnisse unserer Untersuchung zeigen, dass die Leistungsfähigkeit im Zusammenhang mit dem Anbieten von Lernunterstützung steht. Leistungsstarke Schüler/innen weisen demnach häufiger eine helfende Beziehung zu leistungsschwachen Mitschüler/inne/n auf als ihre weniger kompetenten Klassenkolleg/inn/en. Auch wenn dieses Ergebnis vor dem Hintergrund der Annahme, dass nur kompetentere Personen in der Lage sind zu helfen, wenig überraschend erscheint, so ist diese Feststellung dennoch nicht trivial, da bei der Auswahl von Helfer/innen auch andere Kriterien denkbar wären (z. B. Freundschaftsbeziehungen). Die vorgefundenen Lernkonstellationen ermöglichen schwächeren Schüler/inne/n, sich kognitive Fähigkeiten bspw. durch Modelllernen anzueignen. Neben dieser eher kognitiven Funktionsweise wird beim kooperativen Lernen auch auf die nonkognitive, soziale Komponente des Lernens verwiesen (Johnson \& Johnson 1999): Kooperative Lerngruppen weisen eine höhere soziale Kompetenz auf, was wiederum zu positiveren Peer-Beziehungen führen kann. Die vorliegende Studie legt diesen Mechanismus des kooperativen Lernens ebenfalls frei. Sozial kompetentere Schüler/innen zeigen häufiger eine helfende Beziehung $\mathrm{zu}$ leistungsschwächeren Mitschüler/inne/n. Meta-Analysen wie jene von Johnson et al. (1981) bestätigen zwar, dass kooperatives Lernen die soziale Kompetenz fördert und weiterfolgend die Bildung von sozialen Netzwerken begünstigt, jedoch wurde dies bisher noch nicht anhand von relationalen Daten mittels dem Verfahren der Netzwerkanalyse belegt. Dies gilt insbesondere für den deutschsprachigen Raum und für berufsbildende Domänen. Vor diesem Hintergrund gibt die vorgestellte Studie einen tieferen Einblick in die Funktionsweise kooperativen Lernens und bestätigt sozial-konstruktivistische Annahmen über eben diese Form des sozialen Lernens.

\section{Grenzen der Studie und Implikationen für das Lehrerhandeln}

Die teilweise niedrigen Korrelationen lassen sich u. a. auf die Grenzen der Studie zurückführen:

- Die Trennung der Schülerschaft in leistungsschwache und -starke Schüler/innen am Mittelwert bzw. 70. Perzentil ist, wenn auch eine übliche Vorgehensweise, willkürlich.

- Ein weiterer Grund für den geringen Zusammenhang könnte sein, dass sich Lerntandems und -gruppen - nach dem Motto „gleich und gleich gesellt sich gerne“ - häufig homogen in Hinblick auf ihre Kompetenzen bilden (Homophilie-Effekte, McPherson, Smith-Lovin \& Cook 2001).

- Die Erfassung der Sozialkompetenz der Schüler/innen basierte in beiden Fällen auf Selbsteinschätzungsskalen. Ihre Vorteile in Hinblick auf eine 
ökonomische Erhebung gehen auf Kosten der Validität bzw. einer Verzerrung durch ein sozial erwünschtes Antwortverhalten.

- Neben den methodischen Hinweisen ist auch aus theoretischer Sicht kritisch zu hinterfragen, welche individuellen Schülermerkmale über die Leistungsfähigkeit und die Sozialkompetenz hinaus das Anbieten von Lernunterstützung im Unterricht charakterisieren (z. B. die Freundschaftsbeziehung, die Sympathie oder das Geschlecht).

Die Erkenntnisse, die durch die Literaturrecherche und unsere Untersuchung gewonnen werden konnten, lassen darauf schließen, dass der Einsatz von kooperativen Lernformen kein Selbstläufer ist und nicht automatisch eine förderliche Wirkung auf das fachliche Schülerlernen und die sozialen Schülerfähigkeiten entfaltet. Für die Unterrichtspraxis lässt sich aus dem Vorgestellten ableiten, dass insbesondere auf die Rahmenbedingungen für kooperatives Lernen zu achten ist: Unter der Berücksichtigung der von Johnson und Kollegen erarbeiteten Qualitätskriterien kooperativen Lernens ist aus theoretischer Perspektive zu erwarten, dass Peer-Lernen vor allem dann funktioniert, wenn kompetentere Schüler/innen (leistungsfähigere, sozial fähigere) mit jenen zusammenarbeiten bzw. jene unterstützen, die Anleitung und Hilfestellung benötigen, um Aufgaben der nächsten Entwicklungszone zu meistern. Zudem legen die Ergebnisse eine besondere Verantwortung der Lehrkraft für die Bildung heterogener Lerngruppen nahe, die sich nicht nur aufgrund freundschaftlicher Beziehungen oder ähnlichem formieren.

\section{Literatur}

Allport, F. H. (1924). Social Psychology. Boston, MA: Houghton Mifflin.

BMBF. (2014). Lehrplan der Handelsakademie, Bundesministerium für Bildung und Frauen. Zugriff am 05.05.2016. Verfügbar unter https://www.hak.cc/files/ syllabus/Lehrplan_HAK_2014.pdf.

Butts, C. T. (2010). sna: Tools for Social Network Analysis. R package version 2.2-0. Zugriff am 29.11.2016. Verfügbar unter http://CRAN.R-project.org/package=sna.

Carolan, B. V. (2013). Social network analysis and education. Theory, methods \& applications. Los Angeles, California: Sage Publ.

Chi, M. T., Bassok, M., Lewis, M. W., Reimann, P. \& Glaser, R. (1989). SelfExplanations. How Students Study and Use Examples in Learning to Solve Problems. Cognitive Science, 13 (2), 145-182.

Collins, A., Brown, J. S. \& Newman, S. E. (1989). Cognitive apprenticeship: Teaching the crafts of reading, writing, and mathematics. In L. B. Resnick (Eds.), Knowing, learning, and instruction: Essays in honor of Robert Glaser (S. 453494). Hillsdale, NJ: Erlbaum. 
Drössler, S. (2010). Förderung sozialer Kompetenzen im Unterricht-Prozessorientierte Evaluation eines Fortbildungsprojekts für die Sekundarstufe I. Dissertation. Institut für Erziehungswissenschaften. Berlin: Humboldt Universität.

Eberle, F., Schumann, S., Oepke, M., Müller, C., Barske, N., Pflüger, M. et al. (2009). Instrumenten- und Skalendokumentation zum Forschungsprojekt Anwendungsund problemorientierter Unterricht in gymnasialen Lehr-/Lernumgebungen $(A P U)$. Zürich: Universität Zürich, Institut für Gymnasial- und Berufspädagogik.

Euler, D. (2016). Sozialkompetenzen - allseits gefordert, wenig erforscht. Zeitschrift für Berufs- und Wirtschaftspädagogik, 112 (4), 517-524.

Euler, D., Jüttler, A., Raatz, S. \& Schumann, S. (2016). Förderung von Teamkompetenzen durch angeleitete Reflexionsprozesse in schulischen Gruppenarbeiten. Zeitschrift für Berufs-und Wirtschaftspädagogik, 4 (112), 611-635.

Euler, D., Pätzold, G. \& Lang, M. (2005). Förderung selbst gesteuerten und kooperativen Lernens in der beruflichen Erstausbildung. Das neue Modellversuchsprogramm SKOLA hat begonnen. Die berufsbildende Schule, 57 (6), 137-140.

Euler, D., Pätzold, G. \& Walzik, S. (2007). Zeitschrift für Berufs-und Wirtschaftspädagogik. Kooperatives Lernen in der beruflichen Bildung (Bd. 21). Stuttgart: Franz Steiner Verlag.

Gillies, R. M. \& Ashman, A. F. (2003). Co-operative Learning: The Social and Intellectual Outcomes of Learning in Groups. London: Routledge Falmer.

Gräsel, C., Stark, R., Sparka, A. \& Herzmann, P. (2007). Schulische Kooperationsmuster und die Implementation eines Programms zur Förderung der Lesekompetenz. Zeitschrift für Berufs- und Wirtschaftspädagogik (Beiheft 21), 93-107.

Gruehn, S. (2000). Unterricht und schulisches Lernen. Schüler als Quellen der Unterrichtsbeschreibung. Münster: Waxmann.

Güzel, E., Nickolaus, R., Zinn, B., Würmlin, J. \& Sari, D. (2016). Soziale Kompetenzen von angehenden Servicetechnikern - Relevanz, Förderung und Ausprägungen. Zeitschrift für Berufs- und Wirtschaftspädagogik, 4 (112), 555-583.

Hattie, J. (2009). Visible Learning. New York: Routledge.

Helm, C. (2016). Berufsbildungsstandards und Kompetenzmodellierung im Fach Rechnungswesen. In Bundesinstitut für Berufsbildung (Hrsg.), Bildungsstandards und Kompetenzorientierung. Herausforderungen und Perspektiven der Bildungs- und Berufsbildungsforschung (S. 149-168). Bonn: BIBB.

Ingenkamp, K.-H. (1995). Die Fragwürdigkeit der Zensurengebung. Basel: Beltz.

Johnson, D. W. \& Johnson, R. T. (1987). Learning together and alone: Cooperative, competitive, and individualistic learning. Englewood Cliffs, NJ: Prentice-Hall.

Johnson, D. W. \& Johnson, R. T. (1999). Making Cooperative Learning Work. Theory Into Practice, 38 (2), 67-73.

Johnson, D. W. \& Johnson, R. T. (2008). Wie kooperatives Lernen funktioniert. Über die Elemente einer pädagogischen Erfolgsgeschichte. Friedrich Jahresheft, 26 (XXVI), 16-20.

Johnson, D. W., Maruyama, G., Johnson, R., Nelson, D. \& Skon, L. (1981). Effects of Cooperative, Competitive, and Individualistic Goal Structures on Achievement: A Meta-Analysis. Psychological Bulletin, 89 (1), 47-62.

KMK. (2004). Rahmenlehrplan für den Ausbildungsberuf Kaufmann im Einzelhandel/Kauffrau im Einzelhandel, Kultusministerkonferenz. Zugriff am 28.11.2016. Verfügbar unter http://www.kmk.org/fileadmin/Dateien/pdf/Bildung/Berufliche Bildung/rlp/KfmEinzelhandel.pdf. 
Konrad, K. (2014). Lernen lernen - allein und mit anderen. Konzepte, Lösungen, Beispiele. Wiesbaden: Springer Fachmedien.

Kunter, M., Schümer, G., Artelt, C., Baumert, J., Klieme, E., Neubrand, M. et al. (2002). PISA 2000: Dokumentation der Erhebungsinstrumente. Berlin: MPI.

Krause, U.-M. (2007). Feedback und kooperatives Lernen. Münster: Waxmann.

Maier, U. (2012). Lehr-Lernprozesse in der Schule: Studium. Regensburg: Klinkhardt.

McPherson, M., Smith-Lovin, L. \& Cook, J. M. (2001). Birds of a feather: Homophily in social networks. Annual review of sociology, 27, 415-444.

Nickolaus, R., Heinzmann, H. \& Knöll, B. (2005). Ergebnisse empirischer Untersuchungen zu Effekten methodischer Grundentscheidungen auf die Kompetenzund Motivationsentwicklung in gewerblich-technischen Berufsschulen. Zeitschrift für Berufs und Wirtschaftspädagogik, 101 (1), 58-78.

Quellenberg, H. (2009). Studie zur Entwicklung von Ganztagsschulen (StEG) - ausgewählte Hintergrundvariablen, Skalen und Indices der ersten Erhebungswelle. Frankfurt am Main: Gesellschaft zur Förderung Pädagogischer Forschung.

R Core Team. (2014). R: A language and environment for statistical computing. $R$ Foundation for Statistical Computing, Vienna, Austria. Zugriff am 29.11.2016. Verfügbar unter http://www.R-project.org/.

Renk1, A. (1997). Lernen durch Lehren. Zentrale Wirkmechanismen beim kooperativen Lernen. Wiesbaden: Deutscher Universitätsverlag.

Riemann, R. \& Allgöwer, A. (1993). Eine deutschsprachige Fassung des „Interpersonal Competence Questionnaire“ (ICQ). Zeitschrift für differentielle und Diagnostische Psychologie, 14 (3), 153-163.

SBFI. (2011). Verordnung des SBFI über die berufliche Grundbildung. Staatssekretariat für Bildung, Forschung und Innovation. Zugriff am 28.11.2016. Verfügbar unter http://www.freisschulen.ch/files/user_upload/BiVo_68500_def.pdf.

Seidel, T. (2014). Pädagogische Psychologie. Weinheim: Beltz.

Sembill, D. \& Seifried, J. (2006). Selbstorganisiertes Lernen als didaktische LehrLern-Konzeption zur Verknüpfung von selbstgesteuertem und kooperativem Lernen. Zeitschrift für Berufs- und Wirtschaftspädagogik, (Beiheft 20), 93-108.

Shaw, M. E. (1932). A Comparison of Individuals and Small Groups in the Rational Solution of Complex Problems. The American Journal of Psychology, 44 (3), 491-504.

Skvoretz, J. (10.02.2015). Handling missing data in social network analysis. (E-Mail).

Slavin, R. E. (1993). Ability Grouping in the Middle Grades: Achievement Effects and Alternatives. The Elementary School Journal, 93 (5), 535-552.

Slavin, R. E. (1995). Cooperative learning: Theory, research, and practice. Boston: Allyn and Bacon.

Trost, S. \& Weber, S. (2012). Fähigkeitsanforderungen an kaufmännische Fachkräfte - Eine kompetenzbasierte Analyse von Stellenanzeigen mittels O*NET. Zeitschrift für Berufs- und Wirtschaftspädagogik, 108 (2), 217-242.

Tschöpe, T. \& Monnier, M. (2016). Modellierung, Messung und Förderung sozialkommunikativer Kompetenzen von Medizinischen Fachangestellten in der Ausbildung: Ableitungen aus dem Projekt CoSMed. Zeitschrift für Berufs- und Wirtschaftspädagogik, 112 (4), 525-554.

Vygotsky, L. S. (Hrsg.). (1978). Mind in society. The development of higher psychological processes. Cambridge, Mass.: Harvard Univ. Press. 
Watson, G. (1928). 'Do groups think more efficiently than individuals'. Journal of Abnormal Psychology, 17, 328-36.

Wellenreuther, M. (2010). Lehren und Lernen - aber wie? Empirisch-experimentelle Forschungen zum Lehren und Lernen im Unterricht. Baltmannsweiler: Schneider-Verl. Hohengehren.

Wölfer, R. \& Cortina, K. S. (2014). Die soziale Dimension der Lernmotivation Netzwerkanalytische Untersuchung schulischer Zielorientierungen. Zeitschrift für Erziehungswissenschaft, 17 (5), 189-204.

Zander, L., Kolleck, N. \& Hannover, B. (2014). Editorial Note. Zeitschrift für Erziehungswissenschaft, 17 (5), 1-7. 



\title{
Inklusion als fachdidaktischer Anspruch
}

\author{
Manuela Niethammer und Anke Langner
}

\section{Motive und Faktoren inklusiven Lehrens}

Inklusion zielt auf die Teilhabe aller Menschen an gesellschaftlichen Prozessen unabhängig von ihren individuellen Dispositionen. Nach diesem Begriffsverständnis, welches sowohl auf die Forderungen der UN-Behindertenrechtskonvention als auch auf die Leitlinien für Bildungspolitik der Deutschen Unesco-Kommission (DUK) rekurriert, lässt sich Inklusion ,nicht ausschließlich auf die Differenzlinie der Behinderung" (Amrhein 2016, 19) und ebenso wenig auf das Wirkungsfeld von Schule reduzieren. Vielmehr sind alle gesellschaftlichen Bereiche zu öffnen und durch die „Strukturen selbst so zu gestalten und zu verändern, dass sie der realen Vielfalt menschlicher Lebenslagen - gerade auch von Menschen mit Behinderung - von vornherein gerecht werden" (Aichele 2010, 15, zitiert in Amrhein 2016, 19).

In dieser inklusiven Gesellschaft ist die inklusive Berufsbildung ein Teilsystem, dem der Auftrag zukommt, den Zugang aller Menschen zur gesellschaftlichen Teilhabe mittels beruflicher Teilhabe zu unterstützen. Die Umsetzung der inklusiven Schule geht mit Gestaltungsbedarfen auf allen Ebenen der Bildungsgestaltung einher, wobei sich diese wechselseitig beeinflussen (Buchmann \& Bylinski 2013 sowie Bylinski 2016, vgl. Abbildung 1).

Im Folgenden wird aus Sicht der Didaktik der Beruflichen Fachrichtungen $^{1}$ auf die Mikroebene der Bildungsgestaltung (vgl. die beiden unteren Ebenen in Abbildung 1) fokussiert und der Frage nachgegangen, welche fachdidaktischen Optionen gegeben sind, um Lehr-/Lernprozesse so zu gestalten, dass die Partizipation aller Lernender bestmöglich unterstützt und gefördert wird. Letztere lässt sich nicht auf die ,gemeinsame Beschulung von Schülerinnen und Schüler mit und ohne Förderbedarf reduzieren und ausschließlich durch additive Maßnahmen für die Menschen mit Behinderung realisieren." (Amrhein 2016, 19) Vielmehr geht es darum, jeden Einzelnen, unabhängig von seinen individuellen Dispositionen, an der kognitiven und emotionalen Auseinandersetzung mit der Lebens- und Arbeitswirklichkeit aktiv zu beteiligen. Damit wird auch der Forderung der EFA-Bewegung (Education for All) entsprochen, wonach ,die Konzepte inklusiver Bildung

1 Im berufsbildenden Kontext wird der Begriff der Didaktik beruflicher Fachrichtungen verwendet (KMK 2008), da keine Bezugsfächer für berufliches Lernen existieren. Zur Vereinfachung wird nachfolgend der Begriff Fachdidaktik synonym verwendet. 
und qualitativ hochwertige Bildung immer mehr miteinander in Verbindung zu bringen sind“" (DUK 2009, 12, vgl. auch Bylinski 2016). Die Sicherung qualitativ hochwertiger Bildung muss mit Veränderungen in den Inhalten, fachdidaktischen Ansätzen, Strukturen und Strategien (DUK 2009, 10 ff.) sowie mit veränderten Haltungen dazu einhergehen (Langner 2015).

Abb. 1: Gestaltungsanforderungen auf allen Ebenen des Berufsbildungssystems

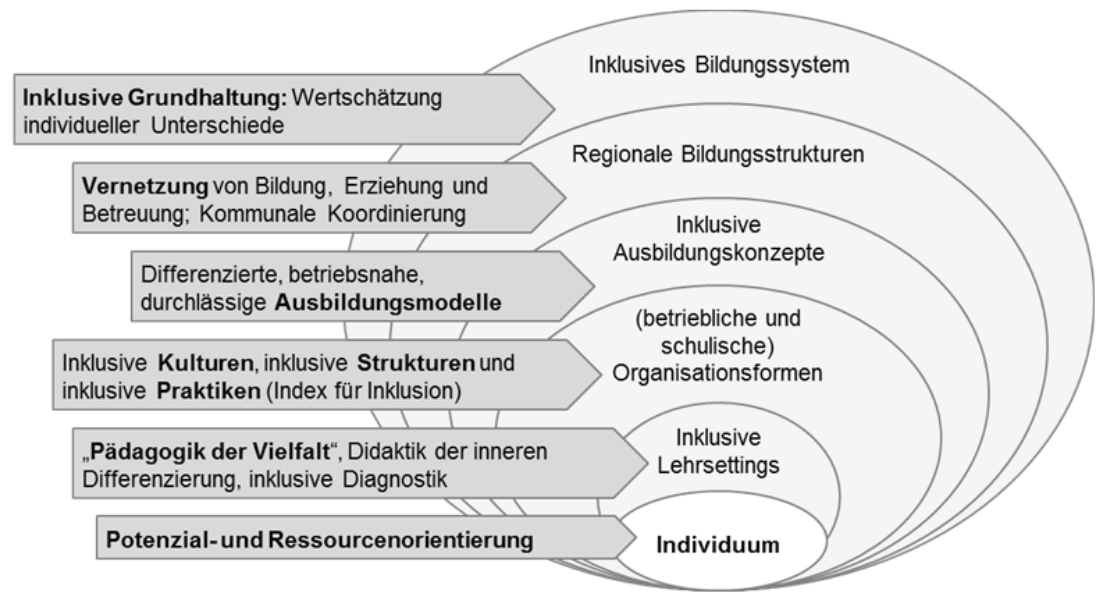

Quelle: Bylinski 2016, 6

Die unakzeptable Zahl von Ausbildungsabbrüchen (2013 wurden $25 \%$ der Ausbildungsverträge vorzeitig gelöst, siehe BiBB-Datenreport 2015, Tab. A4.7-2) bzw. von Menschen ohne Ausbildung (13,5\% der Alterskohorte der 20-34-Jährigen blieben 2013 ohne Abschluss, ebd. Tab. A8.3-1) spricht dafür, dass die Partizipation aller Auszubildenden nicht durchgängig gelingt. Unter diesem Gesichtspunkt ist die Qualität beruflicher Bildung zu hinterfragen. Zur Beantwortung der Frage nach den Optionen fachdidaktischen Handelns werden sowohl sonderpädagogische als auch berufsdidaktische Ansätze geprüft und aufeinander bezogen.

In den vorliegenden sonderpädagogischen bzw. allgemeindidaktischen Modellen (vgl. Amrhein \& Reich 2014; Feuser 1989, 2011; Ziemen 2014) wird postuliert, dass Individualisierung und Differenzierung maßgebend für die Wirksamkeit fachlichen Lernens in heterogenen Gruppen sei. Diesbezügliche Studien verweisen auf individualisierte Curricula sowie auf einen Unterricht, der in Kooperation sowohl bezogen auf die Schüler_innen als auch auf die Lehrer_innen stattfindet (vgl. Kullmann et al. 2014). Individualisierung und Differenzierung als die wesentlichen Merkmale inklusiven Unterrichts lassen sich ebenso über den o. g. Anspruch begründen, dass alle Ler- 
nenden gemäß ihren Lernausgangslagen an der kognitiven und emotionalen Auseinandersetzung beteiligt werden sollen. Um den verschiedenen Interessen und Voraussetzungen zu entsprechen, sind differenzierte und individuelle Lernwege notwendig.

Wie jedoch diesem Anspruch aus fachdidaktischer Perspektive Rechnung getragen werden kann, scheint vorerst nicht vollständig beantwortet und lässt sich als Forschungsdesiderat kennzeichnen (vgl. Werning \& Arndt 2015; Buchmann \& Bylinski 2013). Der Diskurs zur Gestaltung inklusiver Berufsausbildung orientiert vor allem auf die Meso- und Makroebene der Bildungsgestaltung (Buchmann \& Bylinski 2013; Euler \& Severing 2015; Bylinski \& Rützel 2016; Zoyke \& Vollmer 2016). Gestaltungsoptionen für das fachdidaktische Handeln unter den Bedingungen einer inklusiven Berufsschule werden jedoch kaum diskutiert.

Hier setzt der Beitrag an und diskutiert fachdidaktische Gestaltungsspielräume zur Umsetzung inklusionsbezogener beruflicher Lehr-Lern-Settings. Dies erfolgt in Reflexion der Modellierung vom Lernprozess. Daraufhin werden die Ansätze der Sachstruktur- und Handlungsstrukturanalyse im Sinne der lehr-lerntheoretischen Didaktik (vgl. Heimann, Otto \& Schultz 1966) sowie in ihrer kritischen Reflexion in der Entwicklungslogischen Didaktik (Feuser 1989) nach ihren Potenzialen für die Individualisierung und Differenzierung von Lernwegen hinterfragt.

\section{Bezugspunkte fachdidaktischen Handelns}

\subsection{Lernen als Bezugspunkt fachdidaktischen Handelns}

Grundlage didaktischer Überlegungen ist das Verständnis vom Lernprozess. Der unmittelbare Lernprozess kann dadurch charakterisiert werden, dass das Individuum ,auf der Basis seiner Sensibilität gegenüber der Umwelt [...] über die ,Brücke der Wahrnehmung' Informationen aus der Außenwelt aufnimmt, speichert und integriert und durch Rekombination des Erfahrenen selbst neue Information im System schafft und nach dieser Erfahrung handelt" (Feuser 1989, 21). Der Mensch generiert hierüber interne Repräsentationen vom jeweils betrachteten Ausschnitt der Welt und schafft sich damit neue innere Strukturen, Denk- und Handlungsmöglichkeiten (vgl. Hascher \& Astleitner 2007, 26). 
„Das innere Abbild ${ }^{2}$ ermöglicht die vorgreifende Widerspiegelung in Bezug auf die umgebende Welt, d. h. es ermöglicht die Antizipation des Kommenden und damit eine stabile Orientierung des Individuums auf und in seine Lebenswelt" (Feuser 1989, 22). Über die innere Repräsentation wird folglich die Sensibilität gegenüber der Umwelt und darüber die Wahrnehmung maßgeblich beeinflusst. Durch die wechselseitigen Abhängigkeiten der verschiedenen Lernphasen ist der Lernprozess eher als ein schleifenförmiger als ein linearer Prozess zu verstehen, welcher kognitive, emotionale und motivationale Operationen impliziert (vgl. Hascher \& Astleitner 2007, 26). Der so verstandene Lernprozess charakterisiert nach Feuser eine horizontale Dimension des komplexen menschlichen Aneignungsprozesses. Das Schema in Abbildung 2 veranschaulicht, dass Wissenserwerb und Erkenntnisprozesse mit der Wahrnehmung realer oder repräsentierter Wirklichkeit korrespondieren, wobei diese aus dem Kontext sinnstiftender Tätigkeit heraus stattfindet (Feuser 2011, 93).

\section{Abb. 2: Vereinfachtes Schema zum Lernprozess}

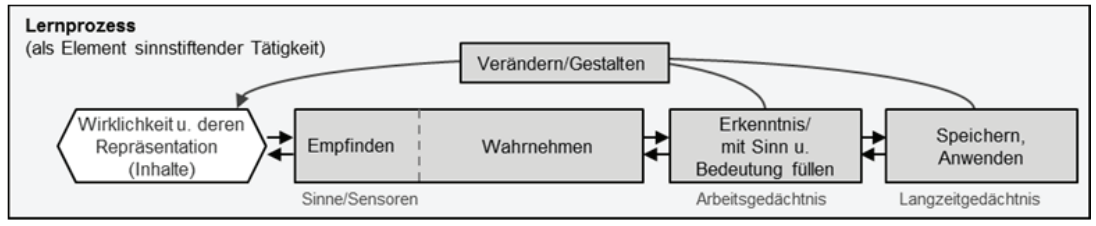

Quelle: vgl. u.a. Rubinstein 1958; Vollmer 2002; Feuser 1989, Renkl 2002, Schnotz \& Bannert 2003

\subsection{Die Sachstrukturanalyse als Bezugspunkt fachdidaktischen Handelns}

Der didaktischen Unterrichtsplanung und -umsetzung werden verschiedene didaktische Modelle zugrunde gelegt. Bezogen auf den Anspruch inklusiven Unterrichts stellt sich die Frage, ob spezielle Modelle nötig sind oder ob es nicht vielmehr einer differenzierten, feindiagnostischen Anwendung der didaktischen Konzepte der Beruflichen Fachrichtungen bedarf.

Im fachdidaktischen Diskurs zu inklusiven Lehr-Lern-Settings wird häufig die Entwicklungslogische Didaktik Feusers zugrunde gelegt, die im Kontext der Behindertenpädagogik entworfen wurde (vgl. Riegert \& Musenberg 2015). Charakteristisches Merkmal ist die Forderung, Unterrichten konse-

2 Der Begriff des inneren Abbildes entspricht dem der inneren Repräsentation. Da der AbbildBegriff missverständlich ist und eine subjektunabhängige Widerspiegelung suggerieren könnte, wurde er kritisch diskutiert und im Begriff der inneren Repräsentation, welcher auch von Feuser (2011) genutzt wird, aufgehoben. 
quent an der menschlichen Entwicklung und dem Lernen auszurichten (Feuser 1998, 2011). Den didaktischen Ansätzen nach Blankertz (1971), der bildungstheoretischen (Klafki 1985, 1996) und lerntheoretischen Didaktik (Heimann, Otto \& Schultz 1966) weist Feuser eine dominant einseitige Orientierung auf die Sachstruktur zu und spricht ihnen insofern ab, den Anspruch der Individuumzentriertheit zu erfüllen (Feuser 1989). Diese kritische Haltung gegenüber der Sachstrukturanalyse prägt die Diskussion um inklusionsbezogene Lehr-Lern-Settings, in der der Subjektorientierung eine Vorrangstellung eingeräumt wird (ebd.).

Aus fachdidaktischer Sicht ist das Verhältnis von Subjekt und Sache (als Aneignungsgegenstand) jedoch als ein dialektisches zu verstehen (Klingberg 1996), weshalb es wenig zielführend ist, die Dominanz einer Seite der didaktischen Grundbeziehung herauszustellen. Dieses Zusammenspiel wird auch bei Feuser deutlich: „Eine entwicklungslogische Didaktik hätte [...] die Frage zu beantworten, welche sachstrukturellen Momente sich ein Kind in der handelnden Auseinandersetzung mit diesen sinnbildend aneignen und im Sinne der Ausdifferenzierung interner Repräsentationen ein qualitativ neues und höheres Wahrnehmungs-, Denk- und Handlungsniveau anbahnen und absichern kann. Damit ,dienen ' die Sachverhalte, Inhalte und Gegenstände der Auseinandersetzung [...] der Persönlichkeit" (Feuser 2011, 93).

Es handelt sich um die Dialektik zwischen subjektiver und gesellschaftlicher Entwicklung, die sich im Wechselverhältnis von individueller Bedeutungszuschreibung und gesellschaftlicher Bedeutung zeigt (vgl. Rubinstein 1959). Letztere meint und umfasst das gesamte gesellschaftlich verfügbare Wissen und Können. „Die Behandlung dieses Verhältnisses [...] ist im Bildungsprozess ein elementares Problem der Didaktik bzw. eine elementare Aufgabe an die Didaktik. [...] (Individuelles) Lernen und (gesellschaftliches) Erkennen (sind, d. A.) als im Verhältnis stehend, als vermittelt aufzufassen. Dabei ist das ,Logische‘, das sich im Prozess der historischen Entwicklung des Erkennens herausgebildet hat, [...] auch das Gemeinsame, das sowohl die historische Entwicklung der (gesellschaftlichen) Erkenntnis wie den Prozess des (individuellen) Lernens miteinander verbindet.“ (Rubinstein 1958, zitiert in Dafermos \& Marvakis 2009, 84 f.). Die gesellschaftliche Bedeutung und die innewohnende „Logik“ der „Sache“ sind folglich wesentliche Bezugspunkte für das didaktische Handeln. Über die Sachstrukturanalyse sind sie zu rekonstruieren, um Lernpotenziale der „Sache“, variable Zugänge zu diesen und mögliche Lernhürden offenzulegen. Dies ist die Basis, um individualisierte und differenzierte Lernangebote zu generieren.

Die Aufarbeitung der Inhalte als Aneignungsgegenstand der Lernenden setzt voraus, dass die sachstrukturelle Seite zunächst in ihrer innewohnenden Logik - unabhängig vom Subjekt - analysiert wird. Hierfür ist ein vertieftes Verständnis der Fachinhalte notwendig (vgl. sachlogische Strukturierung der Inhalte, Storz \& Wirsing 1987, sowie specialized content knowledge, Ball 
2000), welches durch das Wissen über die zentralen domänenspezifischen Konzepte (Begriffe) und deren Relationen charakterisiert ist. Erst dieses vertiefte Verständnis befähigt dazu, die Potentiale eines Inhaltes hinsichtlich der Ermöglichung individualisierter Lernwege und lernförderlicher Bedingungen zu erkennen (Hill, Ball \& Shilling 2008).

Zur Unterstützung der Lehrenden werden allgemeine Schemata, die die zentralen domänenspezifischen Konzepte und deren Relationen zueinander skizzieren, bereitgestellt. In ihnen werden die Inhaltsstrukturen oberhalb der Beispielebene operationalisiert (vgl. Abbildung 3). Studien im universitären Setting bestätigen positive Effekte für die Planung kognitiv aktivierenden Unterrichts, wenn Studierende mit diesen Schemata von Fachwissensstrukturen vertraut gemacht werden (Frank 2016).

Auch wenn die sachlogische Struktur der Inhalte zunächst in ihrer objektiven Logik erschlossen werden muss, ist der Analysefokus im Interesse der didaktischen Fragestellung einzuengen. Bezugspunkt ist nicht das wissenschaftlichen Verständnis zu den Inhalten (wie Feuser 1989 kritisch anmerkt) sondern die Frage, welches Wissen die sachgerechte Bewältigung der jeweiligen beruflichen Arbeitsaufgaben fundiert ${ }^{3}$ (vgl. Niethammer 2006). Die übergeordneten Initiierungskontexte der Aufgaben, wie Arbeitsauftrag, Arbeitssituation, Rechercheauftrag, sind dabei mitzudenken, da sie das relevante arbeitsaufgabenbezogene Wissen mitbestimmen (vgl. Niethammer 2006, 86).

Das relevante Wissen wird in handlungs- und fachbezogenes Wissen unterteilt (vgl. Theorie der Handlungsregulation, Hacker 1986). Im Rahmen der Kompetenzforschung wurden die Begriffe des Handlungs- und Sachwissen etabliert (vgl. Franke 2001). Handlungswissen steht dabei für Wissen über zieladäquate, einander bedingende Handlungen bzw. Operationen zur Bewältigung der Arbeitsaufgabe (ebd., 17). Jede Handlung kann als Prozesseinheit aufgefasst werden, welche durch eine definierte Änderung am Arbeitsgegenstand gekennzeichnet ist. Sie bilden die Bezugsgröße für die Zuordnung des Sachwissens, über das das Handlungswissen generiert oder begründet werden kann. Sachwissen im bautechnischen Kontext umfasst Fakten, Sachverhalte und Zusammenhänge zu mindestens folgenden Aspekten der Prozesseinheit: ${ }^{4}$

3 Infolge der Forderung nach wirksamer Kompetenz- und Handlungsorientierung wurde in den 90er Jahren das Lernfeldkonzept als curricularer Ansatz für die schulische Berufsausbildung implementiert. Kennzeichend ist, dass berufliche Handlungen bzw. Arbeitsaufgaben zu Bezugspunkten beruflichen Lernens werden. An die Stelle der Fächer traten Lernfelder, welche zusammengehörige berufliche Aufgabenkomplexe repräsentieren (Bader 2000). Das Prinzip der Handlungsorientierung ist für die allgemeine Kompetenzorientierung zu übertragen, womit andere Kriterien der Inhaltsauswahl zu beachten wären. In diesem Beitrag wird der Blick auf die berufliche Kompetenzentwicklung eingeengt.

4 Unabhängig davon kann das im Rahmen der konkreten Arbeitsaufgabe relevante Sachwissen Gegenstand verschiedener Fachwissenschaften (Bauchemie, Konstruktionslehre, Baubetrieb etc.) sein und entsprechend unterschiedlich fachsystematisch strukturiert vorliegen. 
- Arbeitsgegenstände (z. B. Funktion und Konstruktion des zu bauenden Elements, Aufbau und Eigenschaften der Baustoffe),

- Mechanismen und Wirkungen natürlicher und technologischer Prozesse, die zu erwünschten (z. B. Aushärten von Beton) oder unerwünschten (Carbonatisierung) Änderungen am Arbeitsgegenstand führen, inklusive der Prozessbedingungen (z. B. Temperatur, Druck, Feuchte) sowie

- Funktionsweise und Handhabung der Arbeitsmittel (z. B. handgeführte Geräte, Baumaschinen) inklusive nötiger Hilfsmedien, Arbeitsschutz etc.

Für die Strukturierung der Inhalte im Kontext von Arbeitsaufgaben werden entsprechende Schemata (vgl. Abbildung 3) - verschieden nuanciert - in den Didaktiken der Beruflichen Fachrichtungen diskutiert (vgl. Niethammer 2006; Becker, Fischer \& Spöttl 2010).

Die objektive Bedeutsamkeit von handlungs- und fachbezogenen Wissen korreliert mit den konkreten Arbeitsanforderungen. Aufgaben, die über Routinen und auf Basis handlungsbezogenen Wissens zu bewältigen sind, stellen Mindestanforderungen eines Berufs dar. Mit zunehmenden Anforderungen steigt das Maß des erforderlichen fachbezogenen Wissens, z. B. wenn Handlungsroutinen bedingungsadäquat anzupassen sind. Handlungs- und fachbezogenes Wissen sind dann aufeinander zu beziehen. Danach können Arbeitsaufgaben graduiert werden in:

- Routineaufgaben als Aufgaben, die regelgeleitet zu bewältigen sind, so dass das Handlungswissen eine ausreichende Basis für die sachgerechte Bewältigung der Aufgabe bietet.

- Problemhaltige Aufgaben als Aufgaben, bei denen aufgrund unvollständig definierter Ausgangslagen oder äußerer Einflüsse, wie tektonische, klimatische, technologische Bedingungen auf der Baustelle, der bedingungsadäquate sachgerechte Handlungsablauf erst generiert werden muss, was sowohl Handlungs- als auch Sachwissen erfordert.

- Optimierungs- bzw. Entwicklungsaufgaben als Aufgaben, bei denen gewohnte Handlungsabläufe in Frage zu stellen und neu zu gestalten sind. Diese Aufgaben erfordern Handlungs- und Sachwissen und sind gemeinhin im Team zu bewältigen (vgl. Eberhardt \& Schlegel 2011).

Zudem variieren die schwierigkeitsbestimmenden Merkmale des Sach- und Handlungswissen, wie Komplexität oder die notwendige Abstraktionsebene des Wissens (vgl. Bernholt 2010; Kauertz 2008).

Die Analyse des im Kontext beruflicher Arbeitsaufgaben relevanten Wissens bietet sowohl die Grundlage für die individualisierte Differenzierung inhaltlicher Anforderungen im Rahmen des arbeitsaufgabenbasierten Lernens als auch für die Bewertung der individuell erschlossenen Inhalte (Handlungswissen vs. Kopplung von Sach- und Handlungswissen in unterschiedlicher Tiefe und Breite). 
Abb. 3: Ansatz zur Strukturierung bildungsrelevanter Inhalte im Kontext beruflicher Arbeitsaufgaben

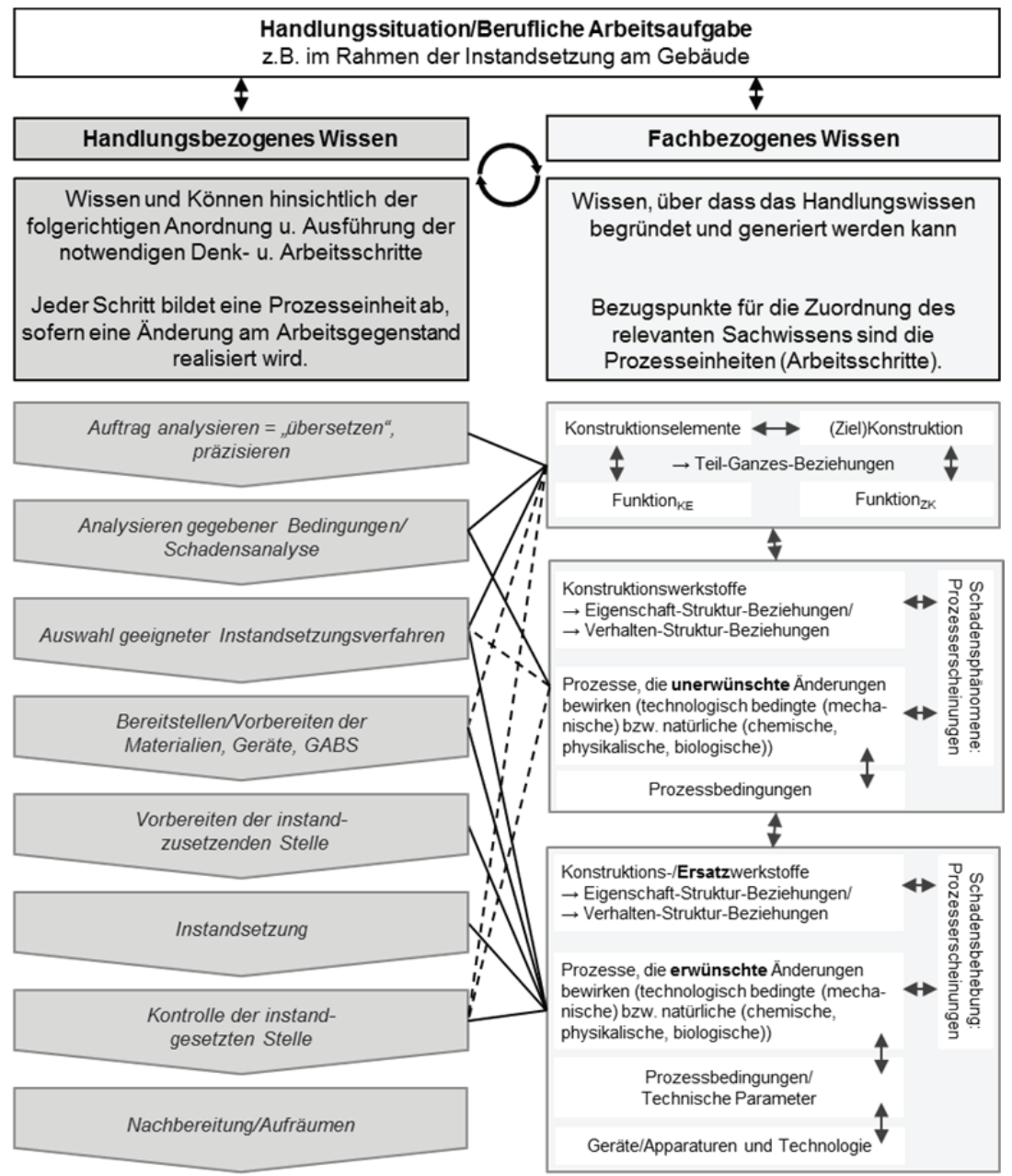

\section{Quelle: Eigene Darstellung}

Mit der Sachstrukturanalyse wird ein Inhaltspotenzial erschlossen, welches die Prognose nötiger sowie die prozessbegleitende Diagnose der gegebenen Wahrnehmungs-, Denk- und Handlungsfähigkeiten der Lernenden fundiert (vgl. bei Feuser Handlungsstrukturanalyse). Durch eine Marginalisierung der 
Sachstrukturanalyse wird der didaktischen Gestaltung die Basis entzogen. Die Gestaltung von Lehr-Lern-Settings liefe Gefahr, beliebig zu werden.

\subsection{Die Vorwegnahme hypothetischer Lernprozesse als Bezugspunkt fachdidaktischen Handelns}

Die ermittelten Inhaltspotenziale implizieren Lernmöglichkeiten, welche erst im Handeln von Lehrenden und Lernenden erfahrbar werden. Zur methodischen Gestaltung dieses Prozesses müssen Lehrende die prinzipiellen Herausforderungen, die das Erschließen des jeweiligen Inhalts mit sich bringen kann, vorwegnehmen. Erst daraufhin lassen sich Inhalte als Lerngegenstände bewerten und geeignete Ziel-Inhalts-Methoden-Relationen ableiten, die der Individualisierung des Lernens zugrunde gelegt werden können.

Die Antizipation hypothetischer, inhaltsadäquater Lernhandlungen umfasst die Analyse der Anforderungen, die mit dem Wahrnehmen, dem Denken bzw. dem Erkenntnisprozess, dem Anwenden und Speichern der konkreten Inhalte einhergehen (vgl. Abbildung 2). Durch das Inbeziehungsetzen von Sachstruktur und Lernprozess werden potenzielle Lernbarrieren entlang der Lernphasen prognostizierbar. Das schließt die Reflexion möglicher Fehlkonzepten der Lernenden ein (vgl. z. B. Barke 2006).

Nachfolgend werden exemplarisch Ansprüche dieser Vorwegnahme von Lernprozessen erörtert, wobei ausschließlich die Phase der Erkenntnisgewinnung herausgestellt wird. Die Analyse muss multiperspektivisch für alle Phasen realisiert werden, wobei dann auch explizit die Behinderungen in die Reflexion einzubeziehen sind.

Inhaltsadäquate Erkenntniswege orientieren sich an der Sachlogik. So ist die Schadensanalyse im Rahmen der Instandhaltung daran gebunden, dass der Schaden als unerwünschte Veränderung der bautechnischen Konstruktion verstanden wird und dass Prozesse, die dazu führten, als Grund-Folge-Ketten oder Bedingung-Bedingtes-Zusammenhänge abgeleitet und verifiziert werden können (vgl. Abbildung 3). Das Verstehen einer konstruktiven Lösung oder eines Konstruktionsfehlers setzt voraus, dass die Elemente der Gesamtkonstruktion erkannt und in ihren Funktions-Konstruktions-Zusammenhängen verstanden werden (analytisch-synthetisches Vorgehen). Der Entwurf technischer Lösungsvarianten, z. B. für die Instandsetzung, erfordert dagegen eine Konstruktion gedanklich zu entwickeln, durch die zuvor definierte Funktionen (z. B. Festigkeit) gewährleistet werden können (genetisches Vorgehen).

Im Unterschied zur Auseinandersetzung mit technischen Artefakten erfordert das Verstehen der zugrundeliegenden chemischen oder physikalischen Prozesse (z. B. Korrosion oder Kraftabtrag), dass beobachtbare Phänomene auf deren Ursachen (Mikroprozesse und Mechanismen) zurückge- 
führt werden können. Dies ist an Modellvorstellungen zum Aufbau der Stoffe und deren Verhalten gebunden und schließt deren Entwicklung ein (regressiv-reduktives Vorgehen) (Vollmer 2002). Auf Basis der Modellvorstellungen sind wiederum Voraussagen zum Verhalten der Stoffe, der komplexen technischer Systeme und zu Folgen des eigenen Handelns möglich (progressiv-reduktives Vorgehen) (Storz \& Wirsing 1987).

Die Interdependenzen zwischen Lerninhalten, hypothetischen Lernprozessen und den damit verbundenen Anforderungen für die Lernenden fundieren die komplexe Planung und Umsetzung inklusionsbezogener Lehr-LernSettings, einschließlich der Erstellung der Lehr-Lernmaterialien (lernstrukturelle sowie didaktisch-mediale Hilfen). Hierüber werden Gestaltungsspielräume für die Differenzierung von Lehr- bzw. Lernprozessen transparent.

\section{Fachdidaktische Gestaltungsoptionen für die Differenzierung der Lernwege}

Auf einer ersten Reflexionsebene wird Individualisierung des Lernens über die Inhaltsdifferenzierung fundiert, womit auch eine Zieldifferenzierung einhergeht. Inhaltsdifferenzierung erfolgt durch Auswahl und Zuschnitt der Arbeitsaufgaben oder -situationen als die Wirklichkeitsausschnitte, mit denen die Lernenden mittels Lernaufgaben konfrontiert werden:

- Varianz der Lernpotenziale und -anforderungen: Sind für die thematisierte Arbeitsaufgabe Routinen verfügbar oder sind Handlungspläne zu entwickeln? Erfordert die Aufgabenbewältigung handlungsbezogenes und/oder fachbezogenes Wissen? Welcher Abstraktionsgrad des fachbezogenen Wissens ist für die Problemlösung erforderlich?

- Varianz der Offenheit der Problemlösung: Werden Problementfaltung und -lösung bereitgestellt oder wird die selbständige Problemlösung gefordert? Welche Strukturierungshilfen sowie erkentnisunterstützende Repräsentationen werden zur Verfügung gestellt?

- Varianz der Komplexität der behandelten Arbeitsaufgaben: Wie viele Teilaufgaben bzw. Prozesseinheiten sind für die Lösung der Aufgabe zu bedenken? Wie viele Abhängigkeiten bestehen zwischen diesen?

- Varianz der Inhaltsrepräsentation in der Sinnesmodalität, in der Fokussierung der erkenntnisunterstützenden Merkmale (wesentliche vs. unwesentliche Informationen), im Abstraktionsgrad (Original, erscheinungs-, merkmals-, inaffin)

Auf einer weiteren Reflexionsebene sind Differenzierungsoptionen für die Gestaltung der Lehr-Lernprozesse abzuleiten. Einen ersten Zugang bieten die 
didaktischen Funktionen Motivation/Zielorientierung, Ersterarbeitung, Festigung, Kontrolle, die den Lernprozess spiegeln (vgl. Abbildung 4). Die Kontrolle wird der prozessbegleitenden Diagnostik und Lernbegleitung zugeordnet und gewinnt damit an Reflexivität.

Der Lernprozess wird jedoch aus verschiedenen Perspektiven beeinflusst. Gemäß den Handlungsdimensionen nach Meyer (1987a) können fachdidaktische Gestaltungsspielräume, welche unter Berücksichtigung der individuellen Lernausgangslagen genutzt werden sollten, systematisiert werden:

- Varianz der Lehr-Lern-Schritte und damit der Phasen der Auseinandersetzung in Abhängigkeit des anvisierten Problemlöseprozesses und untergeordnet der Erkenntniswege,

- Varianz der Handlungsmuster (z. B. Recherche, dialogorientiert, experimentelles Erarbeiten) sowie des Selbstständigkeitsgrades (lernstruktureller Hilfen sowie didaktischmedialer Strukturhilfen),

- Varianz der Sozial- und Organisationsformen und damit der Beziehungsstruktur (arbeitsgleich/arbeitsteilig/Stationsbetrieb).

Abb. 4: Didaktische Funktionen als Spiegelung des Lernprozesses

\begin{tabular}{|c|c|c|c|}
\hline \multirow[t]{2}{*}{$\begin{array}{l}\text { Lernprozessbegleitendes } \\
\text { Diagnostizieren }\end{array}$} & \multicolumn{3}{|c|}{ Neigungen, Motivation, Emotionen } \\
\hline & $\begin{array}{c}\text { Interesse, } \\
\text { Wahrnehmungsfahigkeit }\end{array}$ & $\begin{array}{l}\text { Erkenntnisprozess, } \\
\text { Erkenntnisbarrieren }\end{array}$ & $\begin{array}{l}\text { Behaltens- u. An- } \\
\text { wendungsleistungen }\end{array}$ \\
\hline & $4 t$ & $4 t$ & $4 \frac{1}{4}$ \\
\hline \multicolumn{4}{|c|}{$\begin{array}{l}\text { Lernprozess } \\
\text { (als Element sinnstiftender Tätigkeit) }\end{array}$} \\
\hline $\begin{array}{c}\text { Wirklichkeit u. deren } \\
\text { Repräsentation } \\
\text { (Inhalte) }\end{array}$ & $\begin{array}{l:}\text { Empfinden } \\
\end{array}$ & $\begin{array}{l}\text { Erkenntnis/ } \\
\text { mit Sinn u. } \\
\text { Bedeutung füllen }\end{array}$ & $\begin{array}{l}\text { Speichern, } \\
\text { Anwenden }\end{array}$ \\
\hline & Sinne/Sensoren & Arbeitsgedachtnis & Langzeitgedächtnis \\
\hline & $4 t$ & 本支 & 4 \\
\hline \multirow{2}{*}{$\begin{array}{l}\text { Lehren } \\
\text { (als Gestaltung von Rahmen- } \\
\text { bedingungen für Lernen) } \\
\rightarrow \text { hier uber die } \\
\text { Didaktischen } \\
\quad \text { Funktionen }\end{array}$} & \multicolumn{3}{|c|}{ Spiegelung des Lernprozesses in den didaktischen Funktionen } \\
\hline & $\begin{array}{l}\downarrow \\
\text { Motivation/Zielorientierung } \\
\text { u.a. mit dem Ziel der Sensibilisierung } \\
\text { und Sicherung, so dass Reizschwelle, } \\
\text { als Grundlage der Wahrnehmung, } \\
\text { ūberschritten werden kann }\end{array}$ & $\begin{array}{c}\downarrow \\
\text { Ersterarbeitung } \\
\text { u.a. mit dem Ziel der } \\
\text { Systematisierung u. Inter- } \\
\text { pretation der Wahrneh- } \\
\text { mungen; hierūber wird die } \\
\text { kognitive Entwicklung von } \\
\text { Modellvorstellungen } \\
\text { initiiert }\end{array}$ & $\begin{array}{c}\downarrow \\
\text { Festigung } \\
\text { u.a. mit dem Ziel der } \\
\text { kontextunabhăngigen } \\
\text { und anwendungsbe- } \\
\text { reiten Verfügbarkeit } \\
\text { des Wissens }\end{array}$ \\
\hline
\end{tabular}

Quelle: Eigene Darstellung

Die Gestaltungsspielräume sind jeweils für jede Phase des Lernprozesses zu prüfen und zu nutzen. Die Entscheidung, welche Lernwege beschritten, welche Aufgaben und Hilfen gewählt werden, wird im inklusiven Lehr-Lern- 
Setting von den Lernenden selbständig bzw. gemeinsam mit dem Lehrenden in Reflexion der Lernstandserhebung getroffen.

Das Konzept impliziert, dass es keine formale Zuordnung der Auszubildenden $\mathrm{zu}$ einem ,angestrebten Qualifikationsabschluss“ geben muss. In Abhängigkeit der momentanen Wahrnehmungs-, Denk- und Handlungskompetenzen der Lernenden (Zone der aktuellen Entwicklung) sollen sie vielmehr unterstützt werden, die Zone der nächsten Entwicklung anzugehen (Feuser 1989). Hierfür sind die Interdependenzen von Ziel-, Inhalts- und Prozessdifferenzierung auszuloten.

Die erreichten Kompetenzen können für jede_ $n$ Lernende $n$ in den verschiedenen Lernfeldern variieren. Zudem sind unstete Entwicklungsverläufe $\mathrm{zu}$ erwarten, die sowohl beschleunigte Phasen als auch Stagnationen einschließen können. Die prozessbegleitende Diagnostik der Lernstände im Sinne einer formativen Erhebung (= Assessment for learning) bzw. didaktischen Diagnostik (Prengel 2016, 51) ist ein weiteres Handlungsfeld für die Lehrenden, dessen Umsetzung der gesonderten Betrachtung und der Entwicklung entsprechender Instrumentarien bedarf.

Ergänzend zur formativen Erhebung, welche ausschließlich der Lernbegleitung und -unterstützung dient, bedarf es in größeren Abständen einer Bewertung der erreichten Kompetenzstände im Vergleich zur geforderten beruflichen Handlungskompetenz. Sie fundiert die Zuordnung von Qualifikationsabschlüssen, wie Maurer_innen vs. Hochbaufacharbeiter_in.

Eine Individualisierung beruflichen Lernens ist unter Beachtung des Bildungsauftrages der Berufsschule möglich. Sie bedarf der fundierten Sachund Handlungsstrukturanalyse, welche die tradierten didaktischen Handlungsfelder Ziel- und Inhaltsanalyse sowie methodische Gestaltung spiegeln. Hierüber sind fachdidaktische Gestaltungsoptionen theoretisch ableitbar. Für eine Etablierung einer entsprechend inklusionsbezogenen Didaktik der Beruflichen Fachrichtung müssen die hier ausgesparten Analysen ergänzt, Ansätze in der schulischen Praxis erprobt und evaluiert werden.

\section{Literatur}

Amrhein B. (2016). Inklusion als Mehrebenenkonstellation - Anmerkungen zu Rekontextualisierungstendenzen in inklusiven Bildungsreformen. In B. Amrhein (Hrsg.), Diagnostik im Kontext inklusiver Bildung, (S. 17-39). Kempten: Klinkhardt.

Amrhein, B. \& Reich, K. (2014). Inklusive Fachdidaktik. In B. Amrhein \& M. DziakMahler (Hrsg.), Fachdidaktik inklusiv. Auf der Suche nach didaktischen Leitlinien für den Umgang mit Vielfalt in der Schule (S. 31-44). Münster: Waxmann.

Ball, D. L. (2000). Bridging practices - Intertwining concent and pedagogy in teaching and learning to teach. Journal of Teacher Education, 51(3), 241-247. 
Barke, H.-D. (2006). Chemiedidaktik: Diagnose und Korrektur von Schülervorstellungen. Heidelberg: Springer.

Becker, M., Fischer, M. \& Spöttl, G. (Hrsg.) (2010). Von der Arbeitsanalyse zur Diagnose beruflicher Kompetenzen. Frankfurt/M.: Peter Lang GmbH.

Bernholt, S. (2010). Kompetenzmodellierung in der Chemie - Theoretische und empirische Reflexion am Beispiel des Modells hierarchischer Komplexität. Berlin: LOGOS.

BiBB (2016). Datenreport zum Berufsbildungsbericht 2016. Informationen und Analysen zur Entwicklung der beruflichen Bildung. Bonn. http://datenreport. bibb.de/ media2012/bibb_datenreport_2016.pdf(20.07.2016).

Blankertz H. (1971). Theorien und Modelle der Didaktik. München: Juventa Verlag.

Buchmann, U. \& Bylinski, U (2013). Ausbildung und Professionalisierung von Fachkräften für eine inklusive Berufsbildung. In H. Döbert \& H. Weishaupt (Hrsg.), Inklusive Bildung professionell gestalten (S. 147-202). Münster: Waxmann.

Bylinski, U. (1996). Defizite beschreiben - Potentiale erkennen - an den Kompetenzen ansetzen. Zum Kompetenzansatz in der Benachteiligtenförderung. Jugend/Beruf/Gesellschaft. Zeitschrift für Jugendsozialarbeit, 47(1), 32-41.

Bylinski, U. \& Rützel, J. (Hrsg.) (2016). Inklusion als Chance und Gewinn für eine differenzierte Berufsbildung. Bielefeld: W. Bertelsmann Verlag.

Dafermos, M. \& Marvakis, A. (2009). Vermitteltes Erkennen der Welt. Lernen nach Sergej L. Rubinstein. Forum Kritische Psychologie, 53, 71-100. Online: www. kritische-psychologie.de/files/FKP_53_Manolis_Dafermos_Athanasios_ Marvakis.pdf (12.04.2017).

Eberhardt, C. \& Schlegel, B. (2011). Fördert ECVET den Aufbau eines europäischen Mobilitätsverbundes? Das Beispiel CREDCHEM. In: C. Eberhardt (Hrsg.), Mit ECVET zu besserer Mobilität? Von der europäischen Empfehlung zur Erprobung in der Praxis (S. 30-41). Bonn: BIBB.

Euler, D. \& Severing, E. (2014). Inklusion in der beruflichen Bildung. Daten, Fakten, offene Frage. Gütersloh: Bertelsmann Stiftung.

Feuser, G. (1989). Allgemeine integrative Pädagogik und Entwicklungslogische Didaktik. Zeitschrift für Behindertenpädagogik 28(1), 4-48.

Feuser, G. (2011). Entwicklungslogische Didaktik. In A. Kaiser, D. Schmetz, P. Wachtel \& B. Werner (Hrsg.), Enzyklopädisches Handbuch der Behindertenpädagogik-Didaktik und Unterricht, Band 4 (S. 86-100). Stuttgart: Kohlhammer.

Franke, G. (Hrsg.) (2001). Komplexität und Kompetenz. Ausgewählte Fragen der Kompetenzforschung. Bielefeld.

Frank C. (2016). Concept Frameworks in Engineering Education - Is the Students ability to structure engineering content related to their ability to design cognitive challenging tasks. In A. Dershimer, F. Goodale \& A. Mumba (Eds.), Design Charrette to Explore Models for Engaging Science Preservice Teachers in Engineering. Annual International Conference of the NARST, 14.-17.04.2016.

Frank, C., Bernholt, S. \& Parchmann, I. (2016). Modellierung des Zusammenhangs allgemeiner und beruflicher Kompetenzen für die Domäne Chemie. ZfDN, 22 , 43-60.

Hacker, W. (1986). Arbeitspsychologie. Psychische Regulation von Arbeitstätigkeiten. Berlin. 
Hascher, T. \& Astleitner, H. (2007). Blickpunkt Lernprozess. In M. Gläser-Zikuda \& T. Hascher (Hrsg.), Lernprozesse dokumentieren, reflektieren und beurteilen. Lerntagebuch und -portfolio in Bildungsforschung und -praxis (S. 25-43). Bad Heilbrunn: Klinkhardt.

Heimann, P., Otto, G. \& Schulz, W. (1966). Unterricht: Analyse und Planung. Hannover: Schroedel.

Hill, H. C., Ball, D. L. \& Shilling, S. G. (2008). Unpacking pedagogical content knowledge: Conceptualizing and measuring teachers' topic-specific knowledge of students. Journal for Research in Mathematical Education, 39(4), 372-400.

Kauertz, A. (2008). Schwierigkeitserzeugende Merkmale physikalischer Leistungstestaufgaben. Berlin: Logos.

Klafki, W. (1985/1996). Neue Studien zur Bildungstheorie und Didaktik. Zeitgemäße Allgemeinbildung und kritisch-konstruktive Didaktik. Weinheim, Basel: Beltz.

Klieme E. \& Hartig J. (2007). Kompetenzkonzepte in den Sozialwissenschaften und im erziehungswissenschaftlichen Diskurs. In M. Prenzel, I.Gogolin \& H. Krüger (Hrsg.), Kompetenzdiagnostik. ZfE, Sonderheft 8, 11-33.

Klingberg, L. (1996). Lernen - Lehren - Unterricht. Über den Eigensinn des Didaktischen. Online: https://publishup.uni-potsdam.de/opus4-ubp/files/445/KLINGBER.pdf (10.03.16)

KMK (1999). Rahmenlehrpläne für die Berufsausbildung in der Bauwirtschaft, (Beschluss der KMK vom 05.02.1999). Online: www.kmk.org/themen/beruflicheschulen/duale-berufsausbildung/downloadbereich-rahmenlehrplaene.html (21.07. 2016).

KMK (2008). Ländergemeinsame inhaltliche Anforderungen für die Fachwissenschaften und Fachdidaktiken in der Lehrerbildung (Beschluss der KMK vom 16.10.2008 i.d.F. vom 16.03.2017). Online: www.kmk.org/fileadmin/Dateien/ver oeffentlichungen_beschluesse/2008/2008_10_16-Fachprofile-Lehrerbildung.pdf (12.04.2017).

Kullmann, H., Lütje-Klose, B. \& Textor, A. (2014). Eine Allgemeine Didaktik für inklusive Lerngruppen. In A. Bettina \& M. Dziak-Mahler (Hrsg.), Fachdidaktik inklusiv. Auf der Suche nach didaktischen Leitlinien für den Umgang mit Vielfalt in der Schule (S. 89-107). Münster: Waxmann.

Langner, A. (2015). Kompetent für inklusiven Unterricht. Wiesbaden: Springer VS.

Meyer, H. (1987a). UnterrichtsMethoden I: Theorieband. 4. Auflage. Frankfurt/M.: Cornelsen.

Meyer, H. (1987b). UnterrichtsMethoden II: Praxisband. 3. Auflage. Frankfurt/M.: Cornelsen.

Niethammer, M. (2006). Berufliches Lehren und Lernen in Korrelation zur chemiebezogenen Facharbeit. Ansprüche und Gestaltungsansätze. Bielefeld: W. Bertelsmann.

Prengel, A. (2016). Didaktische Diagnostik als Element alläglicher Lehrerarbeit „Formatives Assessment“ im inklusiven Unterricht. In B. Amrhein (Hrsg.), Diagnostik im Kontext inklusiver Bildung (S. 49-64). Kempten: Julius Klinkhardt.

Renkl, A. (2009). Wissenserwerb. In E. Wild \& J. Möller (Hrsg.), Pädagogische Psychologie (S. 3-26), 2. Auflage, Berlin \& Heidelberg: Springer.

Riegert, J. \& Musenberg, O. (Hrsg.) (2015). Inklusiver Fachunterricht in der Sekundarstufe. Stuttgart: Kohlhammer.

Rubinstein, S. (1958/1977). Grundlagen der Allgemeinen Psychologie. Berlin. 
Schnotz, W. \& Bannert, M. (2003). Construction and interference in learning from multiple representation. In Learning and Instruction, 13, 141-156.

Storz P. \&Wirsing G. (Hrsg.) (1987). Unterrichtmethodik Technische Chemie. Berufstheoretischer Unterricht. Leipzig: Dt. Verlag für Grundstoffindustrie.

Vollmer, G. (2002). Evolutionäre Erkenntnistheorie: angeborene Erkenntnisstrukturen im Kontext von Biologie, Psychologie, Linguistik, Philosophie und Wissenschaftstheorie. 8., unveränd. Aufl. Stuttgart, Leipzig: Hirzel.

Werning, R. \& Arndt, A.-K. (2015). Unterrichtsgestaltung und Inklusion. In E. Kiel (Hrsg.), Inklusion im Sekundarbereich. 1. Aufl. (S. 53-98). Stuttgart: Kohlhammer (Inklusion in Schule und Gesellschaft, 2).

Ziemen, K. (2014). Inklusion und deren Herausforderungen für die (Fach)Didaktik. In B. Amrhein \& M. Dziak-Mahler (Hrsg.), Fachdidaktik inklusiv. Auf der Suche nach didaktischen Leitlinien für den Umgang mit Vielfalt in der Schule (S. 4556). Münster: Waxmann.

Zoyke, A. \& Vollmer, K. (Hrsg.) (2016). Inklusion in der Berufsbildung: Befunde Konzepte - Diskussionen. Bielefeld: W. Bertelsmann. 



\section{Individuelle Begleitung beruflicher Entwicklung: Kompetenzförderung anhand von Lernstilen}

Svenja Ohlemann und Katja Driesel-Lange

\section{Individualisierung in der Berufsorientierung}

Zur erfolgreichen Bewältigung berufswahlbezogener Entwicklungsaufgaben im Jugendalter wird Unterstützung benötigt, die z. B. durch Angebote der schulischen Berufsorientierung bereitgestellt wird. Dabei soll dem Umstand Rechnung getragen werden, dass Berufswahl ein längerfristiger Prozess ist, in dessen Verlauf in systematischer Weise Lernerfahrungen ermöglicht werden, die die Entwicklung einer individuellen beruflichen Perspektive fördern.

Um mit Lerngelegenheiten den Berufswahlprozess wirksam zu unterstützen, bedarf es individualisierter Angebote (Whiston, Sexton \& Lasoff 1998; Brown, Ryan Krane, Brecheisen, Castelino, Budisin, Miller et al. 2003), also vor allem entwicklungsangemessener Maßnahmen, die an den entsprechenden Bedürfnissen von Heranwachsenden anschließen können (Driesel-Lange, Kracke, Hany \& Schindler 2013). Denn in Abhängigkeit des berufswahlbezogenen Entwicklungsstandes werden Interventionen in ihrem Nutzen für die eigene Berufsorientierung von Jugendlichen unterschiedlich beurteilt (Driesel-Lange \& Kracke, 2017). Für nicht-individualisierte Maßnahmen lassen sich eher geringe Effekte messen (Ratschinski \& Struck 2008; Ohlemann \& Ittel, 2017) bzw. es profitieren eher die Jugendlichen, die am weitesten in ihrer beruflichen Entwicklung vorangeschritten sind (Driesel-Lange \& Kracke, 2017).

Berufsorientierende Angebote zu individualisieren setzt neben der Diagnostik des Standes der Berufswahl (z. B. Kaak, Kracke, Driesel-Lange \& Hany 2013) auch die Kenntnis und Berücksichtigung weiterer möglicher Einflussfaktoren, wie z. B. des Geschlechts (Kracke \& Driesel-Lange 2016; Faulstich-Wieland \& Scholand 2017) oder, mit Blick auf die pädagogische Konzeption, der präferierten Lernstile (vgl. Kolb \& Kolb 2013) voraus.

Der vorliegende Beitrag beleuchtet die Verknüpfung von Berufswahlkompetenzdimensionen und präferierten Lernstilen als diagnostischem Ausgangspunkt individualisierter Berufsorientierung. Dafür werden zunächst die zwei zugrunde liegenden theoretischen Modelle, nämlich das Thüringer Berufswahlkompetenzmodell (Driesel-Lange, Hany, Kracke \& Schindler 2010a) und ein an Kolb (zfs. Kolb und Kolb 2013) angelehntes Lernstilkonzept entfaltet. Darauf aufbauend wird eine Verknüpfung der beiden Diagnostik- 
instrumente entwickelt. Schließlich werden erste Ergebnisse einer explorativen Studie an weiterführenden Schulen zur praktischen Anwendbarkeit vorgestellt.

\section{Theoretische Fundierung individueller Kompetenzförderung in der Berufswahl}

Um die individuelle Kompetenzförderung in der Berufswahl mehrperspektivisch einzubetten, werden folgend mit dem Konzept der Berufswahlkompetenz (1) und den theoretischen Überlegungen zu Lernstilen (2) zwei theoretische Perspektiven entfaltet.

\subsection{Berufswahlkompetenz (BWK)}

Berufswahlkompetenz gilt im berufswahltheoretischen Diskurs als vieldiskutiertes Konstrukt und wird auch in unterschiedlicher Weise theoretisch modelliert (vgl. z. B. Ratschinski 2014; Hartkopf 2016). Ausgehend von einer berufswahltheoretischen Perspektive, die berufliche Entwicklung als lebenslangen Prozess versteht (Super 1985; Savickas 2005), wird deutlich, dass schulische Berufsorientierung die Entwicklung von Kompetenz(en) anregen soll, die den Einzelnen befähigt, die eigene Berufsbiographie über die gesamte Lebensspanne zu entwickeln (Driesel-Lange et al. 2010a).

Als wichtige Prädiktoren für die erfolgreiche Gestaltung beruflicher Entwicklung gelten nach Herr, Cramer und Niles (2004) selbst- und berufsbezogenes Wissen, Exploration, Planungs- und Entscheidungskompetenzen, Sicherheit und Entschiedenheit, verbunden mit motivationalen und volitionalen Aspekten. Ausgehend von den o. g. entwicklungsorientierten berufswahltheoretischen Perspektiven und empirischen Begründungen sowie kompetenztheoretischen Überlegungen (Weinert 2001; Klieme et al. 2003) gleichermaßen wird individuelle berufliche Entwicklung im Modell zur Berufswahlkompetenz entlang entsprechender Wissensfacetten, motivationaler Aspekte und Handlungserfahrungen im Kontext der Berufswahl beschrieben (vgl. Abbildung 1). Heranwachsende durchlaufen dem folgend einen längerfristigen, individuellen Prozess mit phasentypischen Ausprägungen, in dessen Verlauf sie verschiedene Lern-, Informations-, Bewertungs-, Planungs- und Entscheidungssituationen erfolgreich bewältigen müssen. In diesen erwerben sie selbst- und berufsbezogenes Wissen und wenden dieses an. Immer wieder sind sie dabei aufgefordert, selbstgesteuert und motiviert die Anforderungen, wie z. B. den Abgleich zwischen eigenen Fähigkeiten und dem Anforderungsprofil des Wunschberufs, anzugehen. 
Abb. 1: Berufswahlkompetenzmodell

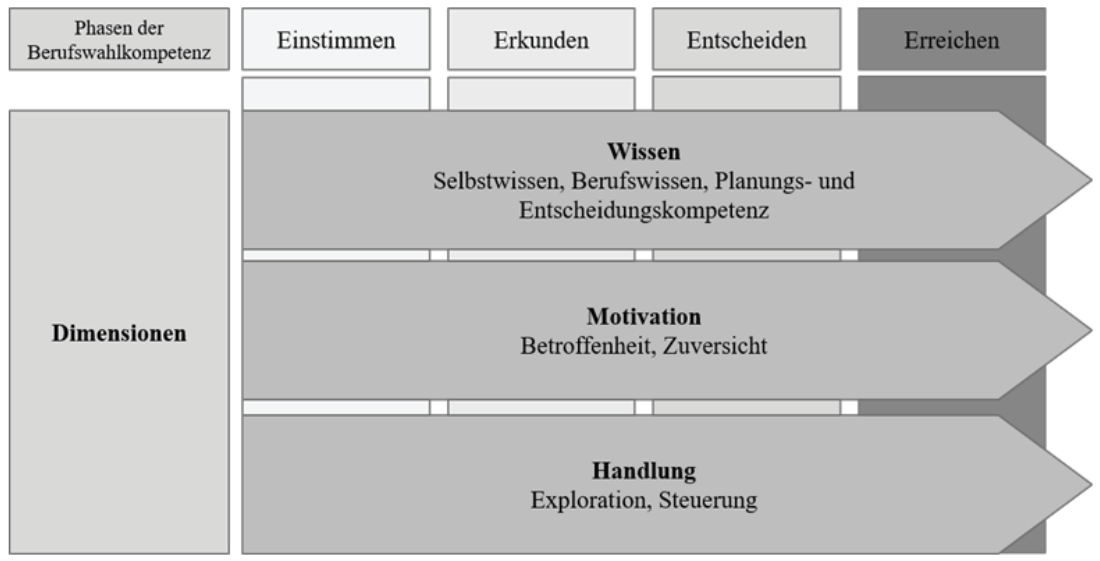

Quelle: Eigene Darstellung nach Driesel-Lange et al. (2010a)

Berufsorientierende Lerngelegenheiten zur individuellen Förderung sind vor diesem Hintergrund in ihrer Konzeption theoretisch bezogen auf ein Verständnis von Berufswahl als selbstgesteuerter Lern- und Entwicklungsprozess, in dessen Zuge Expertise und Kompetenzen erworben werden (vgl. zsf. Driesel-Lange et al. 2010a).

Berufswahlprozesse sind zudem immer in Entwicklungskontexte eingebunden, die einen wesentlichen Einfluss auf Berufspräferenzen wie auch auf die Bereitschaft, sich mit berufswahlbezogenen Fragen zu beschäftigen, haben und damit langfristig erfolgreiche berufliche Entwicklung determinieren (vgl. im Überblick Duffy et al. 2016). Eine individuelle Kompetenzförderung ist z. B. durch die Bereitstellung unterschiedlicher Lernformate pädagogisch umsetzbar. Dabei können u. a. Konzepte individuellen Lernens anhand von Lernstilen (vgl. Kolb \& Kolb 2013) herangezogen werden. Diese sollen folgend vorgestellt werden.

\subsection{Lernstile}

Zhang und Sternberg (2001) definieren den Lernstil als die Präferenz für eine bestimmte Art und Weise des Lernens. Da die individuellen Präferenzen, wie Jonassen und Grabowski (1993) argumentieren, einen wesentlichen Effekt auf das Lernen und Lehren haben (sollten), entstand in den letzten vier Jahrzehnten eine Vielzahl an Lernstilmodellen, zu denen Staemmler (2006) sowie Coffield, Moseley, Hall und Ecclestone (2004b) einen umfassenden Überblick geben. Bezogen auf die existierende Forschung zu Lernstilen bemängeln Pashler, McDaniel, Rohrer und Bjork (2008) das häufige Fehlen eines 
adäquaten Forschungsdesigns, beispielsweise in Form eines ExperimentalKontrollgruppen-Vergleichs. Manolis, Burns, Assudani und Chinta (2013) fassen die umfangreichen Forschungsaktivtäten, die kritisch die Reliabilität und Validität der Skalen des von Kolb erstmals 1976 veröffentlichten Learning Style Inventory (LSI) betrachteten, zusammen. Das LSI wurde im Kontext der Erwachsenenbildung auf Basis der Theorie des Erfahrungslernens entwickelt und erprobt (vgl. Kolb 1984, 1985, zfs. Kolb \& Kolb 2005a).

Das Lernstilmodell in Anlehnung an Kolb (1985), Abbey, Hunt und Weiser (1985) sowie Kolb und Kolb (2013) wird durch zwei Dimensionsachsen bestimmt: Auf der vertikalen Achse ist die bevorzugte Inhaltsvermittlung abgebildet. Sie umfasst das Spektrum zwischen der konkreten Erfahrung (KE), durch Vermittlungsformate mit persönlichem Bezug, und der abstrakten Begriffsbildung $(\mathrm{AB})$, die durch theoriebasierte Lerninhalte intendiert wird. Auf der horizontalen Achse ist die bevorzugte Verarbeitung von Lerninhalten dargestellt: vom aktiven Experimentieren (AE), beispielsweise durch praktisch orientierte Lernformate, bis zur reflektierenden Beobachtung (RB).

Kolbs ursprüngliche vier Learning Styles wurden durch mehrere Erweiterungen und Modifikationen, z. B. durch Abbey et al. (1985), Hunt (1987) und Mainemelis, Boyatzis und Kolb (2002) (vgl. zfs. Staemmler 2006) zu einer Neun-Felder-Matrix weiterentwickelt, die Kolb und Kolb (2013), wie in Abbildung 2 dargestellt, benannten.

\subsection{Berufswahlkompetenz und Lernstile}

Berufswahlkompetenz entwickelt sich langfristig über mehrere Phasen, in denen jeweils entwicklungsangemessene Aktivitäten die Auseinandersetzung mit dem Übergang von der Schule in nachschulische Bildungswege anregen sollen. Lernstile werden durch das Erleben und Verarbeiten verschiedener Erfahrungen sowie die Sozialisation durch Familie, Schule oder Freunde gegebenenfalls beeinflusst (Staemmler 2006).

Wie bereits erörtert, werden Lernstilmodelle in der Forschung kontrovers diskutiert. Dennoch erscheint die Anwendung von Lernstilen als Indikatoren für Aktivitätspräferenzen in der berufsorientierenden Praxis mit der Zielmaßgabe einer Passungsoptimierung zwischen Lernenden und Berufsorientierungsmaßnahmen sinnvoll (vgl. Abbildung 3). Aus der Präferenz eines spezifischen Lernstils ergeben sich Vorlieben für bestimmte Aufgabenformate. Werden diese in den Berufsorientierungskonzepten berücksichtigt, gelingt möglicherweise eine bessere, als passend erlebte Ansprache der Jugendlichen. Ein Maßnahmenangebot, das Lernsituationen initiiert, die zwischen zur Reflektion anregenden Formaten und Aufgaben erprobend-experimentellen Charakters variieren, deckt unterschiedliche Lernstile ab. Dadurch kann es gelingen, Schülerinnen und Schülern variable Angebote zu berufswahlbezo- 
genen Problemstellungen zu eröffnen und auf ihren jeweils präferierten Lernwegen individualsiert die Dimensionen von Berufswahlkompetenz weiter zu fördern.

Abb. 2: Neun-Felder-Matrix in Anlehnung an Kolb (1985), Abbey et al. (1985) und Kolb \& Kolb (2013) ${ }^{l}$

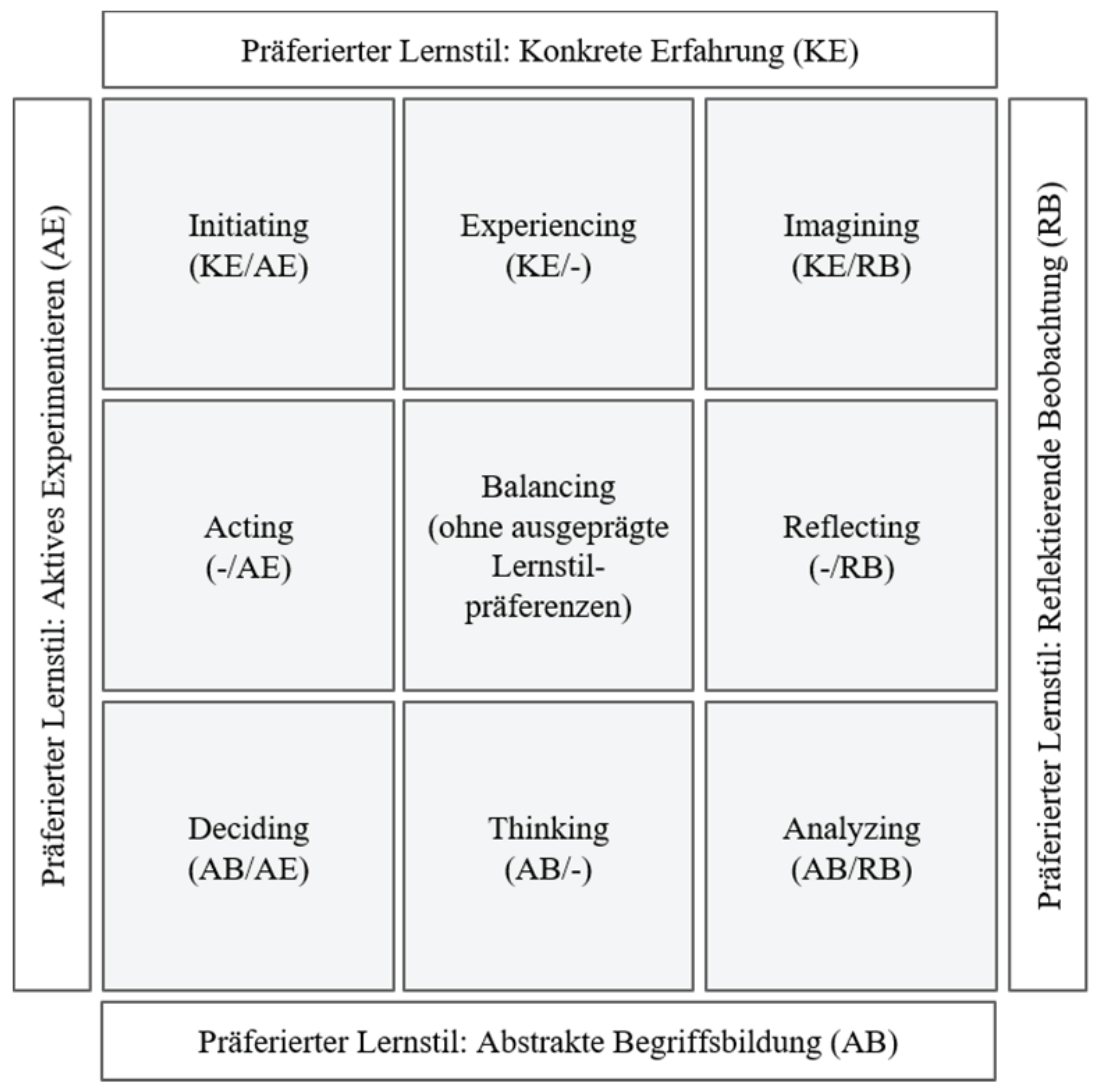

Quelle: Eigene Darstellung

1 Es werden die von Kolb 2013 eingeführten, aufeinander angepassten Bezeichnungen für neun Lernstile genutzt. 
Abb. 3: Spirale lernstilorientierter Berufsorientierung

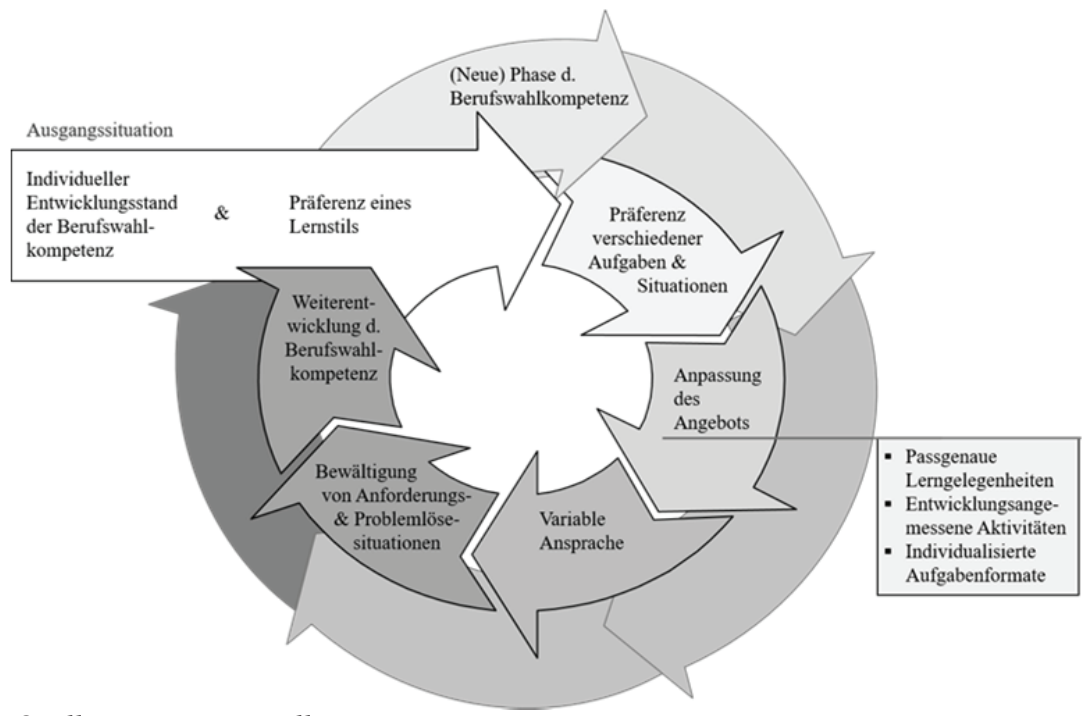

Quelle: Eigene Darstellung

\section{Durchführung der Studie}

\subsection{Fragestellungen}

Das neu konstruierte Modell der Verschränkung von Berufswahlkompetenz und Lernstilen ermöglicht neue Perspektiven auf die praktische Umsetzung individualisierter Berufsorientierung. Zur weiteren Erkenntniserlangung, in welchem Maße der Einbezug von Lernstilen zur Individualisierung schulischer Berufs- und Studienorientierung von Nutzen sein kann, um die theoretisch beschriebenen Effekte auf die Entwicklung der Berufswahlkompetenz $\mathrm{zu}$ provozieren, sollen folgende konkrete Fragen empirisch beleuchtet werden:

1. In welchen Ausprägungen der Berufswahlkompetenz unterscheiden sich Jugendliche?

2. Unter welchen Prämissen lassen sich Kolbs Lernstile auf die Evaluation von Lernpräferenzen Jugendlicher übertragen?

3. Welche Zusammenhänge lassen sich in der Kombination von Lernstilen und der entwickelten Berufswahlkompetenz Jugendlicher beobachten? 


\subsection{Datengrundlage}

Die vorgelegte Untersuchung basiert auf einem gemeinsamen Messzeitpunkt zweier längsschnittlicher Studien: der Berliner Berufs- und Studienorientierungsstudie (vgl. Ohlemann, Ittel, Rohowski \& Lazarides 2016) und dem „Pilotprojekt Potentialanalyse" in Nordrhein-Westfalen (NRW).

In Berlin wurden 296 Lernende der neunten und zehnten Klassen an vier Integrierten Sekundarschulen befragt. An der regionalen Untersuchung in NRW nahmen 216 Heranwachsende der elften Klasse zweier Gymnasien und einer Gesamtschule teil. Die Befragungen fanden im Juni und Juli 2016 als computergestützte Erhebung im Klassenverband während der Unterrichtszeit statt. Es ergibt sich eine Gesamtstichprobe von $\mathrm{N}=521$ Lernenden $(52 \%$ weiblich) der Klassenstufen neun (31\%), zehn (27\%) und elf (42\%). 385 Teilnehmende (76\%) gaben Deutsch als Muttersprache an.

\subsection{Instrumente \& Methode}

Zur Messung der Lernstile wurde eine deutschsprachige Übersetzung von Haller \& Nowack (2010) in einfacher Sprache verwendet. Anhand der vierstufigen Likert-Skalen mit je zehn Items beurteilten die Jugendlichen Lernsituationen und-verhaltensweisen (siehe Tabelle 1).

Tab. 1: Instrumente zur Messung der Lernstile in Anlehnung an Kolb

\begin{tabular}{|c|c|c|c|c|}
\hline Skala (Abkürzung) & Beispielitem & $M W$ & $S D$ & $\alpha$ \\
\hline $\begin{array}{l}\text { Konkrete } \\
\text { Erfahrung (KE) }\end{array}$ & $\begin{array}{l}\text { Ich lerne am besten, wenn es mich } \\
\text { persönlich betrifft. }\end{array}$ & 1.69 & .45 & .73 \\
\hline $\begin{array}{l}\text { Abstrakte } \\
\text { Begriffsbildung (AB) }\end{array}$ & $\begin{array}{l}\text { Ich lerne am besten, wenn ich } \\
\text { Probleme analysieren kann. }\end{array}$ & 1.72 & .50 & .78 \\
\hline $\begin{array}{l}\text { Aktives } \\
\text { Experimentieren (AE) }\end{array}$ & $\begin{array}{l}\text { Ich lerne am besten, wenn ich } \\
\text { Gelegenheit zum Ausprobieren habe. }\end{array}$ & 1.86 & .49 & .77 \\
\hline $\begin{array}{l}\text { Reflektierendes } \\
\text { Beobachten (RB) }\end{array}$ & $\begin{array}{l}\text { Wenn ich lerne, betrachte ich vorher } \\
\text { alle Seiten einer Aufgabe. }\end{array}$ & 1.74 & .47 & .76 \\
\hline
\end{tabular}

$M W=$ Mittelwert $S D=$ Standardabweichung,$\alpha=$ Cronbachs Alpha

Antwortformat: 0 = trifft überhaupt nicht $z u, 3=$ trifft voll $z u$

Quelle: Eigene Darstellung

Die Berechnung der Lernstile erfolgte in drei Schritten: Zuerst wurden die Mittelwerte der Skalen, die im negativen Bereich des Koordinatensystems liegen $(\mathrm{AB}, \mathrm{AE})$, spiegelbildlich negativ rekodiert. Anschließend wurde die 
konkret-abstrakte (aktiv-reflektierende) Dimension aus der Summe der Mittelwerte von $-\mathrm{AB}$ und $\mathrm{KE}(-\mathrm{AE}$ und $\mathrm{RB})$ gebildet. Der daraus entstandene Wertebereich von -3 (äußerste Ausprägung für $\mathrm{AE}$ bzw. $\mathrm{AB}$ ), über 0 (neutral) bis +3 (äußerste Ausprägung für KE bzw. RB) diente der Einteilung der Lernenden in drei Präferenzgruppen $(-1=$ Präferenz für $\mathrm{AE}$ bzw. $\mathrm{AB}$, $0=$ neutraler Bereich, $+1=$ Präferenz für KE bzw. RB) für beide Dimensionen. Anhand der Gruppen konnten, wie Abbildung 4 zeigt, die Lernstile zugeordnet werden.

Abb. 4: Lernstileinteilung anhand der Dimensionswerte

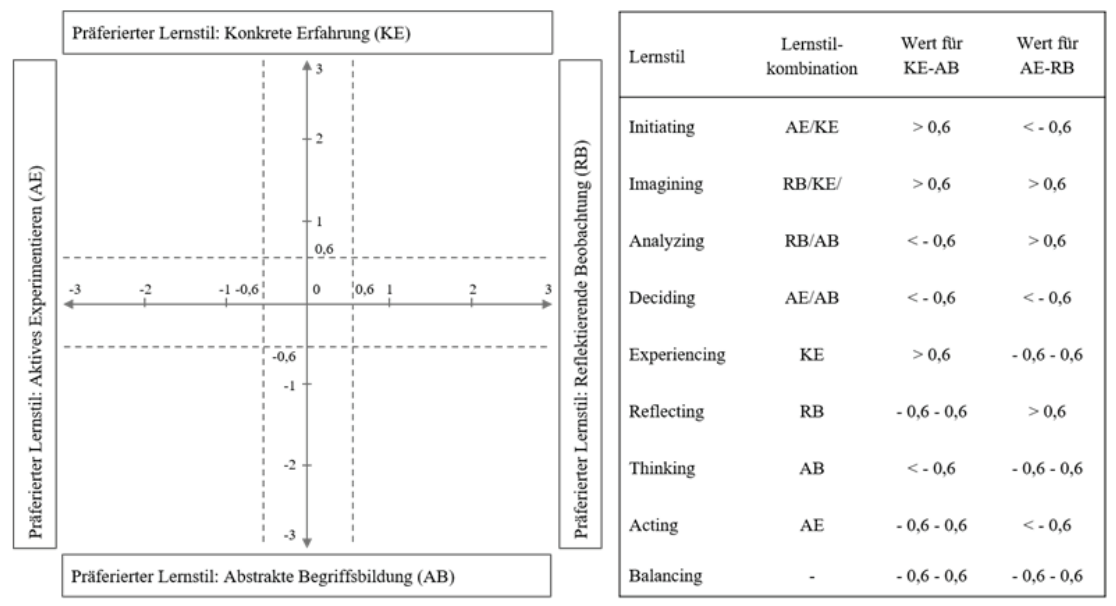

Quelle: Eigene Darstellung

Tabelle 2 gibt eine Übersicht über die sieben, zur Messung der Berufswahlkompetenz (BWK) genutzten, vierstufigen Likert-Skalen. Des Weiteren beantworteten die Jugendlichen Fragen zu ihrem Geschlecht, ihrer Muttersprache, der Klassenstufe und des Schulstandortes.

Zur explorativen Faktorenanalyse wurde eine Hauptkomponentenanalyse mit 40 Items der Learning Style-Skalen mit orthogonalem Rotationsverfahren $(\text { Varimax })^{2}$ berechnet. Die Berechnungen der Two-Step-Clusteranalyse basieren auf dem Log-Likelihood Distanzmaß und dem Schwarzschen BayesKriterium (BIC). Das Silhouettenmaß für Kohäsion und Separation diente zur Evaluation der Clusterqualität.

2 Die Stichprobengröße von $>500$ wird in Bezugnahme auf Tabachnick und Fidell (2012) und Comrey und Lee (1992) für eine Faktorenanalyse als gut eingestuft (vgl. zfs. Field 2009). Das Kaiser-Meyer-Olkin (KMO) Maß bestätigt gemäß Hutcheson und Sofroniou (1999) die Stichprobe als sehr gut bis gut geeignet, $\mathrm{KMO}=.86$ (vgl. ebd.). Auch die individuellen KMO-Werte liegen weit über der akzeptablen Untergrenze von .5 (Field 2013). 
Tab. 2: Instrumente zur Messung der Berufswahlkompetenz (Kaak et al., 2013)

\begin{tabular}{lllll}
\hline Skala (N Items) & Beispielitem & MW & SD & $\alpha$ \\
\hline Selbstwissen (9) & $\begin{array}{l}\text { Ich kann meine Fähigkeiten gut } \\
\text { einschätzen. }\end{array}$ & 3.10 & 0.55 & .85 \\
& $\begin{array}{l}\text { Ich weiß, wie eine betriebliche } \\
\text { Berufsausbildung bzw. ein Studium } \\
\text { Berufswissen (6) }\end{array}$ & 2.61 & 0.67 & .84 \\
& $\begin{array}{l}\text { organisiert ist. } \\
\text { Ich habe mir für die nächste Zeit klare }\end{array}$ & & & \\
Planungskompetenz (3) & $\begin{array}{l}\text { Ziele gesetzt, um dem Berufseinstieg } \\
\text { näher zu kommen. }\end{array}$ & 2.63 & 0.79 & .73 \\
& $\begin{array}{l}\text { Es ist mir wichtig zu klären, was mir } \\
\text { an meinem späteren Beruf einmal } \\
\text { wichtig ist. }\end{array}$ & 3.32 & 0.61 & .89 \\
Betroffenheit (8) & $\begin{array}{l}\text { Wie sehr trauen Sie sich zu, } \\
\text { durchzuhalten, auch wenn es in Ihrer } \\
\text { Ausbildung/Ihrem Studium mal } \\
\text { frustrierend ist? }\end{array}$ & 3.00 & 0.52 & .88 \\
Zuversicht (13) & $\begin{array}{l}\text { Wie oft haben Sie in den letzten } \\
\text { Monaten Informationen über } \\
\text { verschiedene Berufe gesucht? } \\
\text { Wenn ich an einer Sache länger } \\
\text { arbeite, achte ich darauf, dass ich } \\
\text { planmäßig vorankomme. }\end{array}$ & 2.17 & 0.69 & .87 \\
Exploration (9) & & 0.62 & .83 \\
Steuerung (7) & & & & \\
\hline
\end{tabular}

$M W=$ Mittelwert,$S D=$ Standardabweichung, $\alpha=$ Cronbachs Alpha

Antwortformat: 1 = trifft überhaupt nicht $z u, 4=$ trifft voll $z u$

Quelle: Eigene Darstellung

\section{Ergebnisse der Studie}

\subsection{Ergebnisse der Faktorenanalyse bzgl. der Lernstile}

Auf Basis der Faktorenanalyse wurden 21 Items und vier Faktoren beibehalten. Tabelle 3 (siehe Anhang) zeigt die Faktorladungen nach dem Rotationsverfahren. Die vier Faktoren entsprechen den von Kolb formulierten Skalennamen im Groben und tragen zu 47,3 Prozent der Gesamtvarianz bei. Die 
Verteilung der Stichprobe auf die Lernstile ist in Abbildung 5 grafisch dargestellt.

Abb. 5: Verteilung der Stichprobe auf die Lernstile

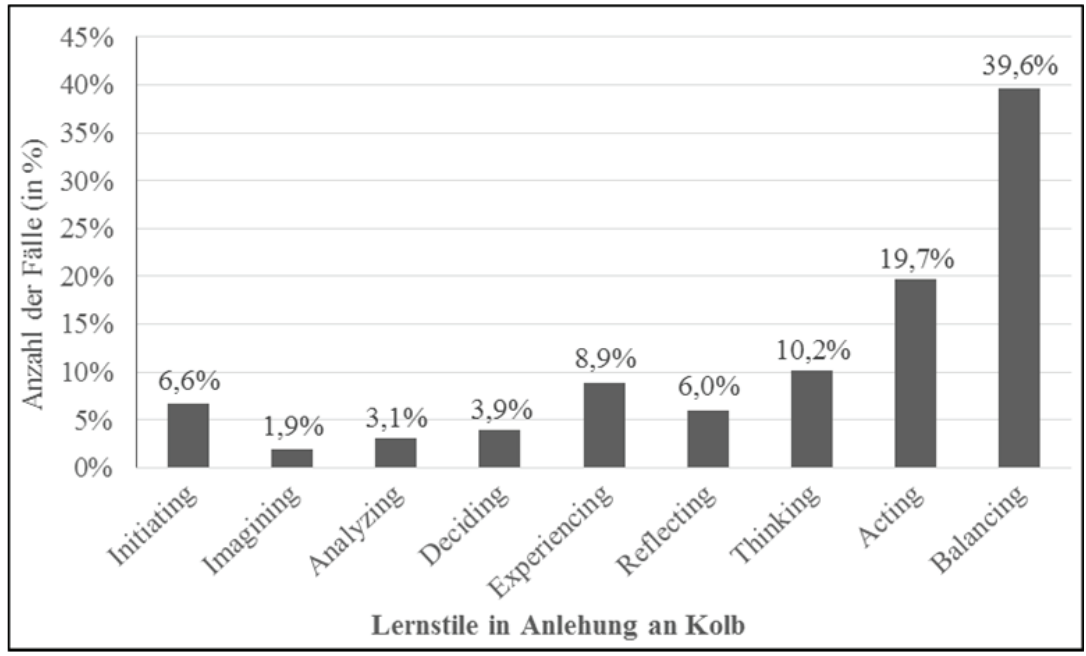

Quelle: Eigene Darstellung

Dabei fällt auf, dass fast 40 Prozent der Schülerinnen und Schüler dem Lernstil Balancing zugeordnet werden können, also keine spezifische Präferenz für einen bestimmten Aufgabentyp oder Verarbeitungsweise haben. Ein Fünftel bevorzugt es, Dinge selbst auszuprobieren, während ein Zehntel sich Inhalte am liebsten über abstrakte Reflektion erschließt.

Die Verteilung der Lernstile unterscheidet sich bezogen auf die Muttersprache und die Klassenstufe der Jugendlichen sowie den Schulstandort nicht. ${ }^{3}$ Jedoch besteht ein signifikanter Unterschied zwischen den Präferenzen von Jungen und Mädchen, $\chi^{2}=(8, N=482)=15.68, p=.047$ : Jungen sind häufiger im Bereich Acting und Deciding vertreten, Mädchen hingegen häufiger im Bereich Reflecting und Analyzing.

\subsection{Ergebnisse der Clusteranalyse zur Berufswahlkompetenz}

Mittels der Clusteranalyse wurde versucht, modellkonform mögliche Strukturen in der Berufswahlkompetenzentwicklung der Heranwachsenden durch die

3 Muttersprache, $\chi^{2}=(8, \mathrm{~N}=482)=11.61, \mathrm{p}=.169$, Klassenstufe, $\chi^{2}=(16, \mathrm{~N}=482)=15.52$, $\mathrm{p}=.487$, Schulstandort, $\chi^{2}=(8, \mathrm{~N}=482)=9.17, \mathrm{p}=.328$. 
Bildung von homogenen Gruppen sichtbar zu machen (vgl. Kaak, Heinrichs, Lipowski, Wuttke \& Kracke 2015). Die ausgewählte Drei-Clusterlösung (vgl. Abbildung 6) zeigte, gemessen am Silhouettenmaß für Kohäsion und Separation, welches in SPSS Werte zwischen -1 (schlechteste) und +1 (beste Clusterqualität) annehmen kann, mit 0,3 eine mittlere Clusterqualität. Zwischen den drei Clustern, weiterhin Phase $1(n=147)$, Phase $2(n=202)$ und Phase 3 $(n=135)$ genannt, bestehen signifikante Unterschiede. ${ }^{4}$ Die Jugendlichen der Phase 3 erreichen auf allen für die Auswertung herangezogenen sieben Kompetenzfacetten Werte, die deutlich über dem theoretischen Skalenmittel und oberhalb der durchschnittlichen Ausprägungen der Gesamtgruppe liegen (vgl. Tabelle 2). Heranwachsende der Phase 1 scheinen am Anfang ihres Entwicklungsprozesses zu stehen. Ihre Kompetenzwerte sind im Vergleich zu den Werten der Phasen 2 und 3 stets niedriger und überschreiten nur in drei Fällen das theoretische Skalenmittel. Die Jugendlichen waren in Bezug auf Geschlecht, Muttersprache und Schulstandort auf alle Phasen gleich verteilt. ${ }^{5}$

\section{Abb. 6: Clusteranalyse. Berufswahlkompetenzentwicklung in den drei Phasen}

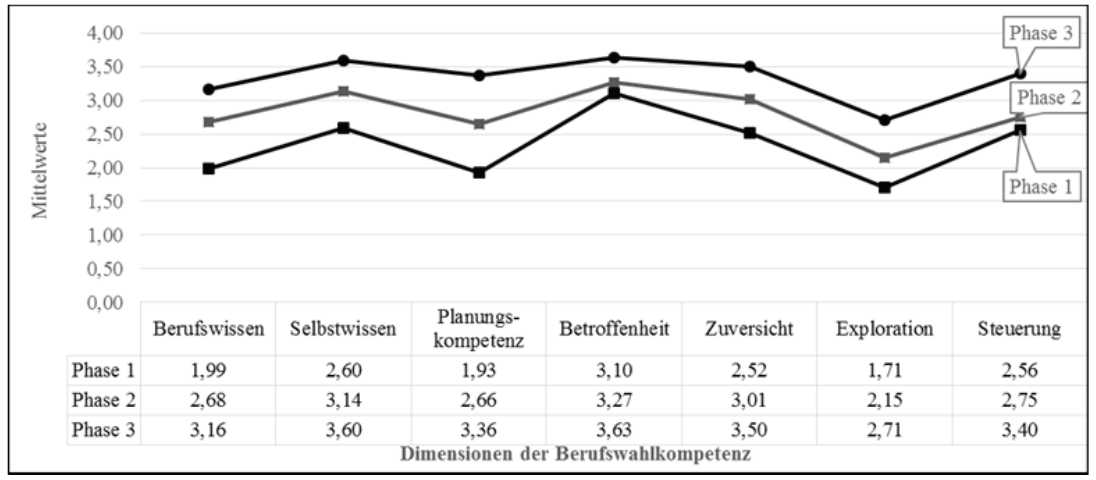

Quelle: Eigene Darstellung

4 Der Kruskal-Wallis-Test zeigt, dass signifikante Unterschiede zwischen den drei Phasen hinsichtlich der Berufswahlkompetenzen bestehen. Die anschließend durchgeführten Posthoc-Tests (Dunn-Bonferroni-Tests) zeigen darüber hinaus, dass sich alle Gruppen signifikant unterscheiden.

5 Geschlecht, $\chi^{2}=(2, N=484)=2.22, p=.313$, Muttersprache, $\chi^{2}=(2, N=484)=5.08, p=$ .079 , Klassenstufe, $\chi^{2}=(4, N=484)=1.72, p=.787$, Schulstandort $\chi^{2}=(2, N=484)=0.6$, $p=.972$ 


\subsection{Ergebnisse zur Verbindung der theoretischen Modelle}

Ein Großteil der Jugendlichen ist über verschiedene Aufgabenformate ansprechbar (vgl. Abbildung 7). Jedoch sind die Lernstile Acting und Experiencing in der ersten Phase besonders häufig vertreten. Initiating ist öfters in der mittleren Phase nachweisbar, wird also von den Jugendlichen präferiert, die sich auf den Weg gemacht haben. Lernende mit einer hohen Affinität zur abstrakten Begriffsbildung, ohne eine besondere Präferenz der Verarbeitung (Thinking) scheinen in allen Phasen in etwa gleich stark vertreten zu sein. Da es sich bei dieser Analyse um eine explorative Betrachtung handelt, in der für einzelne Lernstil-Phasen-Kombinationen (zu) geringe Fallzahlen vorliegen, konnten keine Chi-Quadrat-Tests für die Häufigkeitsverteilung auf die drei BWK-Phasen berechnet werden.

\section{Abb. 7: Kolbs Lernstile im Berufswahlkompetenzmodell}

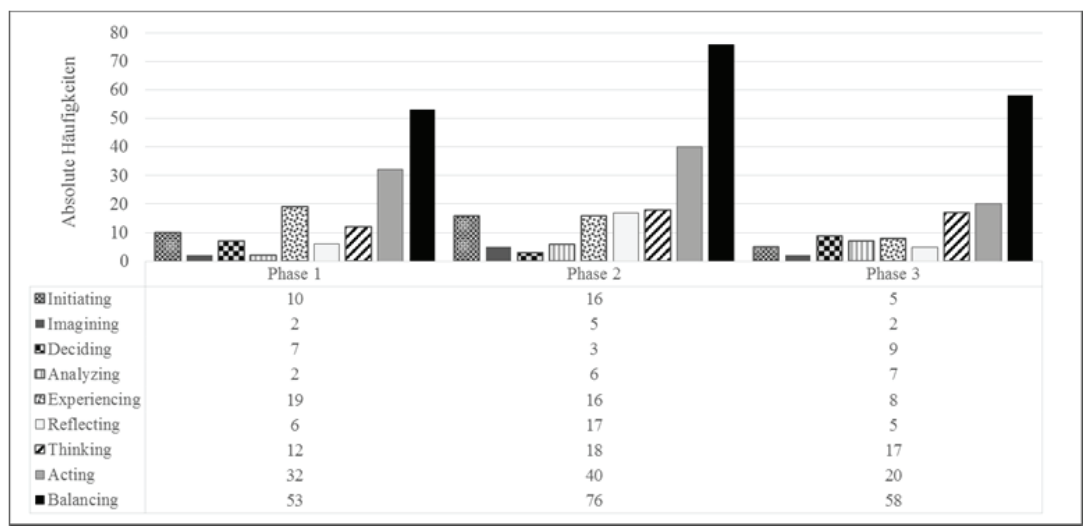

Quelle: Eigene Darstellung

\section{Diskussion}

Auch wenn es sich bei der vorliegenden Untersuchung um eine erste explorative Studie handelt, stellt der Befund, dass auch bei Jugendlichen unterschiedliche Lernstile grundsätzlich differenzierbar sind, einen vielversprechenden Indikator dar. Fast 80 Prozent der Heranwachsenden lassen sich vier der insgesamt neun Lernstile zuordnen. Ein Fünftel der Jugendlichen bevorzugt den Lernstil Acting, hat also eine klare Präferenz für praxisnahe Lernformate. Bezogen auf die Berufsorientierung entsprechen ihnen praxisnahe 
Angebote, wie z. B. das Praktikum, die ein eigenes Ausprobieren zulassen. Knapp 40 Prozent der Heranwachsenden sind Balancing-Lernende. Sie haben (noch) keine Präferenz für einen spezifischen Lernstil, so dass sie sich möglicherweise über eine Vielzahl von berufsorientierenden Instrumenten ansprechen lassen. Etwa jeweils ein Zehntel der Befragten bevorzugt einen der sich gegenüberliegenden Lernstile Experiencing und Thinking.

Während die erste Gruppe insbesondere Vermittlungsformate mit einem persönlichen oder emotionalen Bezug schätzt und durch Interventionen wie z. B. die Potentialanalyse ansprechbar sein könnte, leitet die zweite Gruppe lieber theoretisch allgemeingültige Regeln her. Gerade informationsorientierte Angebote, wie z. B. eigene Internetrecherchen zu berufsbezogenen Themen, entsprechen diesem Lernstil. Es bleibt zu klären, inwiefern sich die Lernstilpräferenzen im Jugendalter verändern. Auch bietet die geringe Varianzaufklärung durch die ausgewählten Items Anlass, die Ergebnisse kritisch $\mathrm{zu}$ betrachten. Eine vergleichbare Studie an englischen Sekundarschulen von Martin (2010) zeigt ebenfalls eine geringe Varianzaufklärung.

Schulform-, Alters- und Klasseneffekte waren in dieser Stichprobe nicht nachweisbar. Jedoch unterschieden sich Jungen und Mädchen leicht in ihren Lernstilpräferenzen. Jungen tendierten häufiger zu einem undifferenzierten Lernstil (Balancing) bzw. dem Bereich des aktiven Handelns und Entscheidens. Mädchen bevorzugten stärker die reflektierende Beobachtung zur Informationsverarbeitung. Diese Befunde sind nicht kongruent $\mathrm{zu}$ früheren LSI-Studien im Erwachsenbereich, in denen Männer häufiger als Frauen im abstrakten Bereich lagen, aber auf der aktionalen Dimension kein Unterschied zwischen den Geschlechtern messbar war (zfs. Kolb \& Kolb 2013). Die empirische Verknüpfung der beiden Messinstrumente ermöglicht erste Annahmen zu den Lernstilpräferenzen in der berufsbezogenen Kompetenzentwicklung. Dabei benötigen die Jugendlichen anscheinend besonders in den ersten beiden Phasen einen starken Lebensweltbezug und die Möglichkeit, Dinge praktisch zu erproben. Im schulischen Kontext eignen sich die Messinstrumente auch zur Reflexion des eigenen Lernens. Sie bieten einen günstigen Ausgangspunkt zu einer Ideenentwicklung für die selbstgesteuerte Bearbeitung nächster Schritte im Berufswahlprozess.

Für eine weiterführende praktische Umsetzung bedarf es jedoch einer konzeptionellen Zuordnung bzw. Entwicklung von Aufgaben und Lernsituationen, die den spezifischen Lernstilen entsprechen. Aufgrund des explorativen Charakters dieser Studie sowie der möglichen Verzerrungen durch die querschnittliche Gelegenheitsstichprobe aus selbstberichteten Antworten der Jugendlichen sollten die Ergebnisse mit Bedacht interpretiert werden.

Zukünftige Studien könnten sich der weiteren Modellierung des Zusammenhangs von Lernstil und der Entwicklung von Berufswahlkompetenz vor allem in Bezug auf weitere Schulformen und Altersgruppen widmen. Auch bedarf es einer weiteren Anpassung des Messinstruments der Lernstile an die 
Zielgruppe der Jugendlichen. So würde möglicherweise eine sprachliche Vereinfachung der Lernstil-Items für die Verwendung im Sekundarschulbereich die Reliabilität der Skalen verbessern. Zudem könnten alternative Lernstilkonstrukte, wie Vermunts Inventory of Learning Styles (1998) oder Allinson und Hayes Cognitive Style Index (1996), herangezogen werden. Beide wiesen in Studien mit Erwachsenen eine hohe interne und Konstruktvalidität sowie eine hohe Test-Retest-Reliabilität auf (vgl. Coffield, 2004a). Darüber hinaus würden Replikationsstudien, längsschnittliche Untersuchungen sowie die von Pashler et al. (2008) geforderten Studien mit Experimental-Kontrollgruppen-Design zur Validierung von Lernstil-Effekten weitere Rückschlüsse auf eventuelle, entwicklungsbedingte Veränderungen in den Lernstilpräferenzen in der Adoleszenz erlauben.

\section{Literatur}

Abbey, D.S., Hunt, D.E. \& Weiser, J.C. (1985). Variations on a theme by Kolb: A new perspective for understanding counseling and supervision. The Counseling Psychologist, 3, 477-501.

Allinson, C. W. \& Hayes, J. (1996). The Cognitive Style Index. Journal of Management Studies, 33, S. 119-135.

Brown, S. D., Ryan Krane, N. E., Brecheisen, J., Castelino, P., Budisin, I., Miller, M. et al. (2003). Critical ingredients of career choice interventions: More analyses and new hypotheses. Journal of Vocational Behavior, 62, 411-428.

Coffield, F., Moseley, D., Hall, E. \& Ecclestone, K. (2004a). Should we be using learning styles? What research has to say to practice. London: LSRC \& Dept. for Education and Skills.

Coffield, F., Moseley, D., Hall, E. \& Ecclestone, K. (2004b). Learning styles and pedagogy in post-16 learning: A systematic and critical review. London: LSRC \& Dept. for Education and Skills.

Comrey, A.L. \& Lee, H.B. (1992). A First Course in Factor Analysis (2.Aufl.). Hillsdale, N.J: Erlbaum Associates.

Driesel-Lange, K., Hany, E., Kracke, B. \& Schindler, N. (2010a). Berufs- und Studienorientierung. Erfolgreich zur Berufswahl. Ein Orientierungs- und Handlungsmodell für Thüringer Schulen. In Thüringer Institut für Lehrerfortbildung, Lehrplanentwicklung und Medien (Hrsg.). Materialien Nr. 165. Bad Berka: Thüringer Institut für Lehrerfortbildung, Lehrplanentwicklung und Medien.

Driesel-Lange, K., Hany, E., Kracke, B. \& Schindler, N. (2010b). Ein Kompetenzentwicklungsmodell für die schulische Berufsorientierung. In U. Sauer-Schiffer \& T. Brüggemann (Hrsg.), Der Übergang Schule - Beruf. Beratung als pädagogische Intervention (S. 157-175). Münster: Waxmann. 
Driesel-Lange, K., Kracke, B., Hany, E. \& Schindler, N. (2013). Das Thüringer Berufsorientierungsmodell: Charakteristika und Bewährung. In T. Brüggemann \& S. Rahn (Hrsg.), Berufsorientierung ein Lehr- und Arbeitsbuch (S. 281-297). Münster [u.a.]: Waxmann.

Driesel-Lange, K. \& Kracke, B. (2017). Potentialanalysen als Instrumente der Förderung in der Berufs- und Studienorientierung. Besondere Herausforderungen der Begleitung von Jugendlichen mit Hochschulzugangsberechtigung. In T. Brüggemann, K. Driesel-Lange \& C. Weyer (Hrsg.), Instrumente der Berufsorientierung (S. 99-124). Münster: Waxmann.

Duffy, R. D., Blustein, D. L., Diemer, M. A. \& Autin, K. L. (2016). The psychology of working theory. Journal of Counseling Psychology, 63, 127-148.

Faulstich-Wieland, H. \& Scholand, B. (2017). Gendersensible Berufsorientierung Informationen und Anregungen. Eine Handreichung für Lehrkräfte, Weiterbildner/innen und Berufsberater/innen. Forschungsförderung Working Paper Nr. 034. Düsseldorf: Hans-Böckler-Stiftung.

Field, A. (2009). Discovering statistics using SPSS: (And sex and drugs and rock'n' roll) (3. Ed.). Los Angeles: Sage.

Field, A. (2013). Discovering statistics using SPSS: (And sex and drugs and rock' $n^{\prime}$ roll) (4. Ed.). London: Sage.

Haller, H. \& Nowack, I.: Lernstildiagnose. Pädagogisches Seminar. Arbeitsblätter. Göttingen. Online: http://mediendidaktik.uni-due.de/sites/default/files/Kolb.pdf (24.05.2016).

Hartkopf, E. (2016). Die Berufswahlreife - Zur Struktur und Relevanz eines vielgesichtigen Konstrukts für die Berufsorientierungs- und Übergangsforschung. Zeitschrift für Berufs- und Wirtschaftspädagogik 112, 1, 60-79.

Herr, E. L., Cramer, S. H. \& Niles, S. G. (2004). Career guidance and counseling through the lifespan: Systematic approaches (6. Ed.). Boston, MA: Pearson.

Hunt, D. E. (1987) Beginning with ourselves in practice, theory and human affairs. Cambridge MA: Brookline Books

Hutcheson, G.D. \& Sofroniou, N. (1999). The Multivariate Social Scientist. London: Sage.

Jonassen, D.H. \& Grabowski, B.L. (1993). Handbook of individual differences, learning and instruction. Hilsdale: Lawrence Eribaum.

Kaak, S., Heinrichs, K., Lipowski, K., Wuttke, E. \& Kracke, B. (2015, 13. März). Der Fragebogen zur Berufswahlkompetenz. Ein Instrument zur Individualisierung der Berufsorientierung? Vortrag auf der 3. Tagung der Gesellschaft für Empirische Bildungsforschung (GEBF), Ruhr Universität Bochum.

Kaak, S., Kracke, B., Driesel-Lange, K. \& Hany, E. (2013). Diagnostik und Förderung der Berufswahlkompetenz Jugendlicher. In K. Driesel-Lange \& B. Dreer (Hrsg.),bwp@Spezial 6-Hochschultage Berufliche Bildung 2013, Workshop 14, 1-13. Online: http://www.bwpat.de/ht2013/ws14/kaak_etal_ws14-ht2013.pdf

Klieme, E.; Avenarius, H.; Blum, W.; Döbrich, P.; Gruber, H.; Prenzel, M.; Reiss, K.; Riquarts, K.; Rost, J.; Tenorth, H.E. \& Vollmer, H.J. (2003). Zur Entwicklung nationaler Bildungsstandards. Eine Expertise. Berlin: BMBF.

Kolb, D. A. (1976). Learning Style Inventory: Technical manual. Boston: McBer \& Company.

Kolb, D. A. (1984). Experiential Learning: Experience as the source of learning and development. New Jersey: Prentice Hall. 
Kolb, D. A. (1985). Learning Style Inventory: Technical manual. Boston: McBer \& Company

Kolb, A. Y. \& Kolb, D. A. (2005a). Learning styles and learning spaces: Enhancing experiential learning in higher education. The Academy of Management Learning and Education, 4, 2, 193-212.

Kolb, A. Y. \& Kolb, D. A. (2005b). The Kolb Learning Style Inventory 3.1: Technical Specifications. Boston, MA: Hay Resources Direct.

Kolb, A. Y. \& Kolb, D. A. (2013). The Kolb Learning Style Inventory - Version 4.0. A Comprehensive Guide to the Theory, Psychometrics, Research on Validity and Educational Applications. Online: http://learningfromexperience.com/media/ 2016/10/2013-KOLBS-KLSI-4.0-GUIDE.pdf (10.09.2016)

Kracke, B. \& Driesel-Lange, K. (2016). Gendersensibilität in der Berufsorientierung durch Individualisierung. In H. Faulstich-Wieland (Hrsg.), Berufsorientierung und Geschlecht (S. 164-185). Weinheim: Beltz Juventa.

Mainemelis, C., Boyatzis, R. \& Kolb, D. A. (2002). Learning styles and adaptive flexibility: Testing experiential learning theory. Management Learning, 33(1), 5-33.

Manolis, C., Burns, D.J., Assudani, R. \& Chinta, R. (2013). Assessing experiential learning styles: A methodological reconstruction and validation of the Kolb Learning Style Inventory. Learning and Individual Differences, 23, 44-52.

Martin, S. (2010). Teachers using learning styles: Torn between research and accountability? Teaching and Teacher Education, 26, 1583-1591.

Ohlemann, S. \& Ittel, A. (2017). Zusammenhänge von Berufs- und Studienorientierungsmaßnahmen und persönlichen Merkmalen von Jugendlichen als Determinanten individualisierter schulischer Berufs- und Studienorientierung? In T. Brüggemann, K. Driesel-Lange \& C. Weyer (Hrsg.), Instrumente der Berufsorientierung (S. 125-151). Münster: Waxmann.

Ohlemann, S., Ittel, A., Rohowski, S. \& Lazarides, R. (2016). Berliner Berufs- und Studienorientierungsstudie (BeBest). Abschlussbericht. Berlin. Technische Universität Berlin, Institut für Erziehungswissenschaft.

Pashler, H., McDaniel, M., Rohrer, D. \& Bjork, R. (2008). Learning Styles: Concepts and Evidence. Psychological Science in the Public Interest, 9(3), 105-119.

Ratschinski, G. (2014).Berufswahlbereitschaft und -fähigkeit als Metakompetenz aus Identität, Adaptabilität und Resilienz. Eine neue Konzeptualisierung der Zielgröße von Berufsorientierungsmaßnahmen, bwp@Berufs- und Wirtschaftspädagogik online, 27, 1-29.

Ratschinski, G. \& Struck, P. (2012). Entwicklungsdiagnostik der Berufswahlbereitschaft und -kompetenz. Konzeptüberprüfungen an Sekundarschülern in einer regionalen Längsschnittstudie, bwp@ Berufs- und Wirtschaftspädagogik - online, 22, 1-18. Online: http://www.bwpat.de/ausgabe22/ratschinski_struck_bwpat22. pdf (23.03.2016).

Savickas, M. L. (2005). The theory and practice of career construction. In S. D. Brown \& R. W. Lent (Hrsg.), Career development and counseling: Putting theory and research to work (S. 42-70). Hoboken, NJ: John Wiley.

Staemmler, D. (2006). Lernstile und interaktive Lernprogramme. Kognitive Komponenten des Lernerfolges in virtuellen Lernumgebungen. Berlin: Springer.

Super, D. E. (1985). Coming of age in Middletown: Careers in the making. American Psychologist, 40, 405-414. 
Tabachnick, B.G. \& Fidell, L.S. (2013). Using multivariate statistics (6. Aufl.). Boston [u.a.]: Pearson.

Vermunt, J. D. (1998). The regulation of constructive learning processes. British Journal of Educational Psychology, 68, 149-171.

Weinert, F.E. (2001). Vergleichende Leistungsmessung in Schulen - eine umstrittene Selbstverständlichkeit. In F.E. Weinert (Hrsg.), Leistungsmessungen in Schulen ( S. 17-31). Weinheim: Beltz

Whiston, S. C., Sexton, T. L. \& Lasoff, D. L. (1998). Career-intervention outcome. A replication and extension of Oliver and Spokane. Journal of Counseling Psychology, 45, 150-165.

Zhang, L.F., Sternberg, R.J. (2001). Thinking styles across cultures: Their relationships with student learning. In R.J. Sternberg \& L.F. Zhang, (Hrsg.), Perspectives on Thinking, Learning, and Cognitive Styles (S. 197-226). London: Lawrence Eribaum. 


\section{Anhang}

Tab. 3: Zusammenfassung der explorativen Faktorenanalyse der Lernstile nach Kolb

\begin{tabular}{|c|c|c|c|c|}
\hline Item & $\mathrm{AB}$ & $\mathrm{AE}$ & $\mathrm{RB}$ & $\mathrm{KE}$ \\
\hline $\begin{array}{l}\text { Ich habe es gern, wenn Dinge systematisch analysiert werden. } \\
\text { (AB) }\end{array}$ & 0,66 & 0,11 & 0,13 & $-0,05$ \\
\hline $\begin{array}{l}\text { Ich bin erst zufrieden, wenn ich etwas ,auf den Begriff“ bringen } \\
\text { kann. (AB) }\end{array}$ & $\mathbf{0 , 5 7}$ & $-0,02$ & 0,15 & 0,14 \\
\hline $\begin{array}{l}\text { Ich lerne am besten, wenn ich mich auf logische Überlegungen } \\
\text { stützen kann. (AB) }\end{array}$ & 0,66 & 0,17 & 0,12 & $-0,02$ \\
\hline Wenn ich lerne, löse ich Probleme durch Nachdenken. (AB) & $\mathbf{0 , 5 2}$ & 0,15 & 0,26 & 0,11 \\
\hline Ich lerne am besten, wenn ich Probleme analysieren kann. (AB) & 0,67 & 0,19 & 0,13 & 0,05 \\
\hline Wenn ich lerne, bin ich jemand, der kritisch bewertet. (AB) & 0,63 & 0,17 & 0,03 & 0,12 \\
\hline Wenn ich lerne, bin ich jemand, der sachlich vorgeht. (AB) & 0,59 & $-0,01$ & 0,31 & 0,14 \\
\hline $\begin{array}{l}\text { Ich orientiere mich eher an Menschen, die in der gleichen Lage } \\
\text { sind wie ich ... (KE) }\end{array}$ & 0,09 & 0,04 & 0,13 & 0,48 \\
\hline Ich lerne am besten, wenn ich mich auf mein Gefühl verlasse. (KE) & 0,02 & 0,07 & 0,10 & 0,74 \\
\hline Ich lerne am besten, wenn es mich persönlich betrifft. (KE) & 0,10 & 0,17 & 0,11 & 0,64 \\
\hline $\begin{array}{l}\text { Ich lerne am besten, wenn meine Spontanität angesprochen wird. } \\
\text { (KE) }\end{array}$ & 0,08 & 0,24 & $-0,10$ & 0,74 \\
\hline $\begin{array}{l}\text { Ich erprobe die Dinge lieber selbst und überzeuge mich davon, was } \\
\text { möglich ist. (AE) }\end{array}$ & 0,20 & 0,51 & 0,20 & 0,14 \\
\hline $\begin{array}{l}\text { Selber zu experimentieren und die Dinge praktisch vorzuführen } \\
\text { erspart viele Worte. (AE) }\end{array}$ & 0,10 & 0,66 & $-0,06$ & 0,07 \\
\hline $\begin{array}{l}\text { Ich lerne am besten, wenn ich Ergebnisse aus meiner Arbeit sehen } \\
\text { kann. (AE) }\end{array}$ & 0,24 & 0,62 & 0,16 & 0,12 \\
\hline Ich lerne am besten, wenn ich praktisch damit umgehen kann. (AE) & 0,10 & 0,78 & 0,16 & 0,12 \\
\hline $\begin{array}{l}\text { Ich lerne am besten, wenn ich Gelegenheit zum Ausprobieren } \\
\text { habe. (AE) }\end{array}$ & 0,04 & 0,79 & 0,16 & 0,16 \\
\hline $\begin{array}{l}\text { Ich halte mich mit Beurteilungen und Stellung-nahmen zurück, bis } \\
\text { ich mir einen Einblick ... (RB) }\end{array}$ & 0,15 & 0,07 & $\mathbf{0 , 5 8}$ & 0,08 \\
\hline $\begin{array}{l}\text { Ich überlege und probiere vorher, wie ich eine Sache angehe, ... } \\
\text { (RB) }\end{array}$ & 0,23 & 0,10 & 0,57 & $-0,01$ \\
\hline Ich lerne am besten, wenn ich zunächst sorgfältig beobachte. (RB) & 0,16 & 0,13 & 0,65 & 0,13 \\
\hline $\begin{array}{l}\text { Wenn ich lerne, betrachte ich vorher alle Seiten einer Aufgabe. } \\
\text { (RB) }\end{array}$ & 0,15 & 0,08 & 0,68 & 0,16 \\
\hline Wenn ich lerne, überlege ich genau, bevor ich handle. (RB) & 0,12 & 0,13 & $\mathbf{0 , 7 3}$ & $-0,03$ \\
\hline Eigenwert & 5,19 & 2,00 & 1,40 & 1,35 \\
\hline$\%$ der Varianz & 24,73 & 9,50 & 6,65 & 6,44 \\
\hline Cronbachs Alpha & .77 & .76 & .71 & .61 \\
\hline
\end{tabular}

Quelle: Eigene Darstellung 


\title{
Beitrag von Banken-Webseiten zur Entwicklung der Finanzkompetenz potentieller Darlehensnehmer/innen über Baufinanzierungen ${ }^{1}$
}

\author{
Mandy Hommel, Bärbel Fürstenau, Claudia Leopold, Héctor Ponce und \\ Mario López
}

\section{Einleitung}

Aus der Perspektive von Finanzdienstleistern wird eine Entscheidung über eine Baufinanzierung traditionell als Entscheidung über die Gewährung eines Darlehens im Anschluss an Beratungsgespräche und an eine Bonitäts- und Kreditwürdigkeitsprüfung verstanden. Diese Perspektive repräsentiert jedoch nur die potentiellen Darlehensgeber. Auch potentielle Darlehensnehmer/innen müssen entscheiden, ob sie ein Darlehen zu spezifizierten Konditionen aufnehmen und damit einhergehend einschätzen, ob sie die kurz- und langfristigen Konsequenzen für ihre persönliche Finanzsituation tragen können und wollen. Im Zuge der Digitalisierung sind viele Informationen, die potentielle Darlehensnehmer/innen früher meist erst in einem Beratungsgespräch erfahren haben, über das Internet verfügbar. Gleichzeitig wird Darlehensnehmer/innen mehr Verantwortung für Entscheidungen übertragen (Aprea, Wuttke, Breuer, Keng Koh, Davies, Greimel-Fuhrmann \& Lopus 2016). Dementsprechend ist es erforderlich, dass potentielle Darlehensnehmer/innen über ein Mindestmaß an Finanzkompetenz im Hinblick auf das in Frage stehende Produkt verfügen.

Wenn sich potentielle Darlehensnehmer/innen vor der Entscheidungsfindung informieren, können Webseiten von Banken als eine der ersten Anlaufstellen angenommen werden. Diese Webseiten scheinen für den genannten Zweck nur bedingt geeignet. So zeigten Fürstenau, Hommel, Leopold, Ponce und López $(2015,2016)$, dass das Informationsangebot von Banken in Bezug auf Qualität und Quantität von Informationen stark variiert und insgesamt nicht voll zufriedenstellend ist.

Vor diesem Hintergrund soll in einer explorativen Studie untersucht werden, ob Online-Informationen von Banken den Erwerb von Wissen über

1 Das Projekt „Cognitive factors and design features that affect understanding of online information associated with financial products: the mortgage case" wurde durch die DFG im Rahmen der Anbahnung einer internationalen Kooperation unterstützt. 
Baufinanzierung und finanzkompetente Entscheidungen potentieller Darlehensnehmer/innen unterstützen. Dazu werden Webseiten zweier Banken ausgewählt, die sich vom Umfang und Qualität der Informationen her unterscheiden. Dadurch können möglicherweise Rückschlüsse auf förderliche oder hinderliche Webseitengestaltung gezogen werden.

\section{Finanzkompetenz im Hinblick auf Baufinanzierung}

Für eine Annäherung an den Begriff der Finanzkompetenz und seiner Spezifizierung im Hinblick auf Entscheidungen über Baufinanzierungen kann der Kompetenzbegriff von Shavelson (z. B. 2010) herangezogen werden. Kompetenzen umfassen gemäß Shavelson $(2010,44)$ verschiedene Facetten: Komplexität, Leistungs-/Performanzbezug, Standardisierung, Realitätsbezug, verschiedene Kompetenzstufen, Entwickelbarkeit sowie die Beeinflussung durch individuelle Dispositionen. Nachfolgend werden diese Facetten hinsichtlich der Finanzkompetenz in Bezug auf Baufinanzierungen interpretiert:

(1) Komplexität: Baufinanzierungen gelten als komplexe Produkte, und Entscheidungen über Baufinanzierungen erfordern komplexe kognitive Fähigkeiten der potentiellen Darlehensnehmer/innen (GSA 2016, 26).

(2) Leistung im Sinne der Wissensanwendung (Performanz): Entscheidungssituationen über den Erwerb einer Immobilie bzw. über die Aufnahme eines Baufinanzierungsdarlehens erfordern von potentiellen Darlehensnehmer/innen, ihr Wissen über finanzierungsrelevante Begriffe und Zusammenhänge anzuwenden. Finanzkompetenz zeigt sich daher insbesondere in solchen Entscheidungssituationen.

(3) Standardisierung: Die im Zusammenhang mit Entscheidungssituationen über Baufinanzierungen zu bewältigenden Aufgaben können vier Kernelementen zugeordnet werden und sind daher für alle potentiellen Darlehensnehmer/innen vergleichbar. Die Kernelemente stellen die zentralen Handlungsbereiche und damit die Anforderungen der Domäne (Seeber 2016, 8) dar. Es handelt sich im Einzelnen um die Folgenden (Fürstenau et al. 2015, 2016): 1. Berechnung des frei verfügbaren monatlichen Einkommens zur nachhaltigen Kapitaldienstleistung, 2. Wohnkostenvergleich der Alternativen Miete und Kauf, 3. Berechnung des Finanzrahmens, der für den Erwerb einer Immobilie zur Verfügung steht und 4. Darlehensberechnung einschließlich Zinssatz, Sollzinsbindung, Laufzeit und Ratenhöhe (Fürstenau et al. 2015, 24 f.). Im Financial Literacy-Begriff der $\operatorname{OECD}$ (2013, 145 ff.) finden sich vergleichbare Fähigkeiten: einen Budgetplan zur Planung und Kontrolle von Einnahmen und Ausgaben aufstellen, die (Wohn)Option mit den geringeren 
Kosten (Mieten oder Kaufen) identifizieren, vorhandenes und neu erworbenes Wissen zu Finanzprodukten anwenden.

(4) Realitätsbezug: Entscheidungssituationen, in denen Finanzkompetenz zum Tragen kommt, sind real erleb-/beobachtbar. Dies gilt in Bezug auf Baufinanzierungen dann, wenn jemand eine Immobilie - (teil-)finanziert über Darlehen - erwerben möchte. Gleichermaßen sind negative Konsequenzen, wie Zwangsversteigerungen von Immobilien erlebbar, was im Zuge der Immobilienblase und der Finanzkrise deutlich wurde.

(5) Verschiedene Kompetenzstufen/-niveaus: Verschiedene Niveaustufen von Finanzkompetenz sind empirisch nachweisbar. Die Kompetenz kann beispielsweise ausreichend oder nicht ausreichend sein. Ausreichend ist sie in dem Fall, in dem Wissen über Kernelemente der Baufinanzierung vorliegt, dieses Wissen in eine begründete Entscheidung einfließt und sich das Finanzierungskonzept im Zeitverlauf als tragfähig erweist. Nicht ausreichend ist sie, wenn zu wenig Wissen vorliegt oder dieses nicht in die Entscheidungsfindung einfließt. Gerardi, Goette und Meier (2010) konnten in ihrer Studie u. a. zeigen, dass die Anzahl von Zwangsversteigerungen bei Personen mit dem höchsten Level numerischer Fähigkeiten (als Aspekt der Finanzkompetenz) um zwei Drittel niedriger war als bei Personen mit geringeren Fähigkeiten.

(6) Entwickelbarkeit: Das Niveau der Finanzkompetenz kann durch Lernprozesse, insbesondere durch Erfahrungen im Umgang mit Geld, Geldanlagen und Finanzierungen, weiterentwickelt und gefördert werden.

(7) Dispositionen: Persönliche und soziale Charakteristika können eine hohe Ausprägung von Finanzkompetenz beeinflussen. Hierzu zählen beispielsweise die Fähigkeit zur Perspektivübernahme, Selbstregulation oder soziale Verantwortung. Finanzkompetente Entscheidungen werden außerdem von Dispositionen wie Risikofreude oder Risikoaversion beeinflusst.

Unter Berücksichtigung dieser konstituierenden Facetten wird im Rahmen dieses Projektes unter der Finanzkompetenz potentieller Darlehensnehmer/innen in Bezug auf Baufinanzierungen zum einen das Wissen über Konzepte, Prozesse und Risiken, die mit Baufinanzierungen verbunden sind, verstanden. Zum anderen geht es um die Anwendung des Wissens in Form informierter und effektiver Entscheidungen, die geeignet sind, die eigene und ggf. die gesellschaftliche Finanzsituation positiv zu beeinflussen (OECD 2013, 144).

Um die Finanzkompetenz in Bezug auf Baufinanzierungen zu messen, wird das Wissen zu einem Kernelement, der Darlehensberechnung, erfasst (Facette 3). Darlehenskalkulatoren beinhalten die zentralen Informationen für die Entscheidungsfindung über ein Baufinanzierungsdarlehen. Gleichermaßen wird die Performanz in den Blick genommen. Diese zeigt sich bei poten- 
tiellen Darlehensnehmer/innen darin, wie sie in realitätsnahen, komplexen Situationen (Facette 4) Entscheidungen für oder gegen die Aufnahme eines Baufinanzierungsdarlehens begründen bzw. rechtfertigen (Facetten 1, 2, 3). Unterschiedliche Niveaustufen von Kompetenz zeigen sich in unterschiedlichen Wissensniveaus und in unterschiedlicher Qualität von Begründungen für oder gegen eine Darlehensaufnahme (Facette 5). In den Entscheidungen für oder gegen ein Darlehen kommt u. a. die Disposition der Risikofreude/aversion zum Ausdruck (Facette 7). Die Möglichkeit zur Entwicklung von Kompetenz wird durch eine Intervention ermöglicht, gemäß der Webseiten von Banken zur gegebenen Thematik exploriert und kognitiv verarbeitet werden (Facette 6). Insgesamt werden damit wesentliche Komponenten von Kompetenz (Shavelson 2013, 75) berücksichtigt.

\section{Methode}

\subsection{Untersuchungsmaterial und Stichprobe}

Die Studie fokussiert die Darlehenskalkulation, d. h. eines der vier Kernelemente der Baufinanzierung (siehe Abschnitt 2). Ausgewählt wurden die Darlehenskalkulatoren der Commerzbank $(\mathrm{CoBa})$ und der NordLB. ${ }^{2}$ Eine Vorstudie (Fürstenau et al. 2015, 2016) zeigte, dass sich die Kalkulatoren der beiden Banken vergleichsweise stark unterscheiden. Der Kalkulator der NordLB umfasst nur wenige Konzepte und bietet zudem keine Erklärungen für die verwendeten Konzepte an. Der Kalkulator der CoBa enthält demgegenüber viele Konzepte und eine vergleichsweise große Anzahl an Erklärungen. In der o. g. Vorstudie wurde untersucht, inwieweit sich die Kalkulatoren der Banken von einem idealtypischen Referenzkalkulator unterscheiden (Fürstenau et al. 2015). Der Referenzkalkulator enthält die zum Verständnis und zur Entscheidungsfindung notwendigen Informationen, d. h. Konzepte und deren Erläuterungen. Er wurde auf Basis der Fachliteratur entwickelt und kommunikativ mit Baufinanzierungspezialisten sowie einem Vertreter einer Verbraucherschutzorganisation validiert. Im Ergebnis zeigte sich, dass die Kalkulatoren beider Banken vergleichsweise weit vom idealtypischen entfernt sind. Der Kalkulator der NordLB weist zwar die geringste Distanz zum

2 Die Braunschweigische Landessparkasse (BLSK) ist Teil der NordLB (als sogenannte „AidA" Anstalt in der Anstalt Norddeutsche Landesbank). 
Referenzkalkulator auf $(0.81){ }^{3}$ allerdings ist der Distanzwert vergleichsweise hoch. Der Kalkulator der Commerzbank (CoBa) weist einen noch höheren Distanzwert von 0.88 auf, stimmt dementsprechend noch weniger mit dem idealtypischen überein. Da sich insbesondere die Darlehenskalkulatoren der NordLB und der CoBa (in Qualität und Quantität) stark voneinander im Sample unterschieden, wurden sie im Hinblick auf ihr Potential zur Unterstützung der Entwicklung von Finanzkompetenz untersucht.

An der Studie nahmen 101 Studierende (Alter $M=23.7$; Tabelle 1) verschiedener wirtschaftswissenschaftlicher Studiengänge teil. Die Studierenden waren Teilnehmer/innen verschiedener Lehrveranstaltungen des fortgeschrittenen Bachelor-Studiums der Wirtschaftswissenschaften und Wirtschaftspädagogik (convenience sample). Sie wurden den Experimentalbedingungen randomisiert zugeordnet.

\section{Tab. 1: Stichprobe}

\begin{tabular}{|c|c|c|c|c|c|}
\hline & & $N$ & $h$ & $M$ Alter & Biolog. Geschlecht \\
\hline Kontrollgruppe (KG) & & 36 & $35.6 \%$ & 26.6 & 9 m, $26 \mathrm{w}, 1 \mathrm{k} . \mathrm{A}$. \\
\hline $\begin{array}{l}\text { Experimentalgruppe } \\
\text { (EG 1, NordLB) }\end{array}$ & 1 & 34 & $33.7 \%$ & 21.9 & $6 \mathrm{~m}, 28 \mathrm{w}$ \\
\hline $\begin{array}{l}\text { Experimentalgruppe } \\
\text { (EG 2, CoBa) }\end{array}$ & 2 & 31 & $30.7 \%$ & 22.9 & $10 \mathrm{~m}, 21 \mathrm{w}$ \\
\hline Gesamt & & 101 & $100 \%$ & & $25 \mathrm{~m}, 75 \mathrm{w}, 1 \mathrm{k} . \mathrm{A}$ \\
\hline
\end{tabular}

Quelle: Eigene Darstellung

\subsection{Erhebungsinstrumente und Untersuchungsablauf}

Die Probanden aller Gruppen bearbeiteten zuerst einen Fragebogen zur Selbsteinschätzung des Finanzwissens und den bisherigen Erfahrungen mit verschiedenen Formen von Geldanlagen und Finanzierungen. Dieser diente dazu, Unterschiede zwischen den Gruppen auf eventuelle Unterschiede in den Erfahrungen zurückführen zu können. Auf einen Vortest (im Hinblick auf Wissens- und Entscheidungsaufgaben) wurde im vorliegenden Fall verzichtet, um den Testing Effect (Roediger \& Karpicke 2006), d. h. einen Lerneffekt durch den Test (und nicht allein durch das Studium der Webseiten)

3 Die Distanz kann Werte zwischen 0 und 1 annehmen, wobei der Wert 0 indiziert, dass keine Distanz besteht, wobei der Wert 1 aussagt, dass die Distanz maximal ist und keinerlei Übereinstimmungen bestehen. Je höher der Distanzwert ist, desto weniger Übereinstimmung besteht mit dem Idealtyp. 
auszuschließen. Danach explorierten die Probanden der beiden Experimentalbedingungen die Darlehenskalkulatoren auf der jeweiligen Webseite der Bank (Facette 6, siehe Abschnitt 2). Die erste Experimentalgruppe (EG 1) nutzte den Darlehenskalkulator der NordLB, die zweite Experimentalgruppe (EG 2) den der CoBa.

Im Anschluss daran wurden die Probanden der EG 1 und EG 2 mit einer komplexen Entscheidungsaufgabe konfrontiert. Um die Entscheidungssituation so realitätsnah wie möglich zu gestalten (Facette 4; siehe Abschnitt 2), wurden zum einen reale Kalkulatoren der Webseiten genutzt sowie zum anderen eine fallbasierte Entscheidungsaufgabe konstruiert, die an einer möglichen zukünftigen Situation der Probanden ausgerichtet ist. Die Probanden erhielten konkrete Informationen zur persönlichen und finanziellen Situation (wie Einnahmen/Ausgaben, vorhandene finanzielle Rücklagen, etc.) einer Kleinfamilie sowie der gewünschten Immobilie (Einfamilienhaus, Kaufpreis und Nebenkosten etc.). Aufgabe der Probanden war es, eine Entscheidung für oder gegen die Aufnahme eines Baufinanzierungsdarlehens zu treffen und diese zu begründen (Facetten 1, 2; siehe Abschnitt 2). Die Entscheidungsfindung schloss die Nutzung des Kalkulators ein. Die erste Entscheidungsaufgabe war so konstruiert, dass der Kapitaldienst auskömmlich tragbar und damit eine Entscheidung für die Darlehensaufnahme möglich war. Dennoch wurde dem möglichen Einfluss individueller Risikodisposition (Risikofreude vs. Risikoaversion, Facette 7, siehe Abschnitt 2) Rechnung getragen, demgemäß sich die Probanden auch begründet gegen eine Darlehensaufnahme entscheiden konnten. Im Anschluss an diese erste Entscheidungsaufgabe erhielten die Probanden eine weitere Entscheidungsaufgabe, die von der ersten im Hinblick auf ein modifiziertes Zinsniveau abwich. Wiederum sollten die Probanden über die Aufnahme des Baufinanzierungsdarlehens entscheiden und ihre Entscheidung begründen (Facetten 2, 5, siehe Abschnitt 2).

Zum Abschluss bearbeiteten alle Probanden (EG 1, EG 2, KG) einen Wissenstest zu relevanten Konzepten und Zusammenhängen der Baufinanzierung bestehend aus 12 Items im MC-Format. Dabei waren jeweils vier Antwortalternativen vorgegeben, aus denen eine richtige oder falsche zu wählen war. Der Test umfasste sowohl Behaltens- als auch Transferaufgaben. Die Behaltensaufgaben dienten zur Einschätzung des Wissens über zentrale Konzepte der Darlehenskalkulation, wie Annuität, Zinsbindung, anfänglicher jährlicher Tilgungssatz. Die Transferaufgaben erforderten die Anwendung des erworbenen Wissens. Beispielsweise mussten die Probanden die Konsequenzen eines veränderten Sollzinses oder einer veränderten Sollzinsbindung für die zu zahlende Summe an Zinsen und Tilgung, die verbleibende Restschuld nach Ablauf der Sollzinszeit sowie mögliche Auswirkungen von Inflation einschätzen. 


\subsection{Auswertung der Daten}

Die Fragebögen zur Selbsteinschätzung wurden einer explorativen Faktorenanalyse unterzogen $\left(N=139,{ }^{4}\right.$ Cronbachs $\alpha=.835, K M O=.918$; VarimaxRotation), die eine Varianzaufklärung von 81.4 Prozent mit einer DreiFaktoren-Struktur zeigte: (1) Erfahrungen mit Geldanlagen und Finanzierungen (Eigenwert 8.7; Varianzaufklärung 54.5\%), (2) Finanzwissen (Eigenwert 2.8; Varianzaufklärung 17.4\%) und (3) subjektive Bedeutung von Wohneigentum (Eigenwert 1.5; Varianzaufklärung 9.5\%).

Die Entscheidungsaufgaben wurden inhaltsanalytisch ausgewertet. Die Argumente der Probanden wurden zunächst erfasst und sowohl untereinander als auch mit einer Referenzargumentation verglichen. Argumente, die inhaltsgleich, aber sprachlich unterschiedlich ausgedrückt waren, wurden vereinheitlicht, um diese Vergleichbarkeit zu ermöglichen. Dies entspricht einer inhaltsanalytischen Kategorisierung (Mayring 2015). Hat beispielsweise ein Proband notiert: "die Sollzinsbindung ist zu kurz" (GA3005) und ein anderer "die Sollzinsbindung beträgt nur 10 Jahre" (KA0411), wurden beide Aussagen unter „Sollzinsbindung ist zu kurz“ kategorisiert. Die Referenzargumente wurden vom Projektteam in Absprache mit einem Baufinanzierungsexperten und unter Bezug auf den validierten Referenzkalkulator der Vorstudie (Fürstenau et al. 2015, 2016, siehe Abschnitt 3.1) gewonnen. Sie umfassen die wesentlichen Begründungszusammenhänge, die für oder gegen eine Finanzierung herangezogen werden können. Dass sowohl Argumente für (Risikofreude) als auch gegen (Risikoaversion) eine Darlehensaufnahme berücksichtigt wurden, trägt der potentiell unterschiedlichen Risikoneigung der Probanden zumindest im Ansatz Rechnung. Um zu ermitteln, wie nah oder weit die Argumentation der Probanden von der Referenzargumentation entfernt ist, wurde für jeden Probanden ein Distanzmaß auf Basis der Galanter-Metrik berechnet (Fürstenau \& Trojahner 2005). Dieses drückt aus, wie ähnlich Probanden- und Referenzargumentation gemessen an übereinstimmenden Argumenten im Verhältnis zu der Gesamtanzahl an Argumenten sind. Es kann als Qualitätsindikator herangezogen werden. Die Distanz kann Werte zwischen 0 und 1 annehmen, wobei der Wert 0 indiziert, dass keine Distanz besteht. Der Wert 1 sagt aus, dass die Distanz maximal ist und keinerlei Übereinstimmungen bestehen. Je höher der Distanzwert ist, desto weniger Übereinstimmung besteht mit der Referenzargumentation.

Die Wissenstests wurden mit einer Musterlösung verglichen. Wurde die richtige Aussage aus den vier Antwortmöglichkeiten gewählt, erhielten die

4 Die Fragebögen wurden einer größeren Stichprobe Studierender vorgelegt, daher konnte für die Faktorenanalyse eine Stichprobe von 139 ausgefüllten Fragebögen genutzt werden. 
Probanden einen Punkt, andernfalls 0 Punkte. Insgesamt konnten sie im Wissenstest 12 Punkte erzielen.

\section{Ergebnisse}

Zunächst werden die Ergebnisse der Wissenstests und daran anschließend die Ergebnisse der Entscheidungsaufgaben dargestellt.

In den Wissenstests erreichen die Probanden der KG mit 5.1 den niedrigsten Mittelwert, den höchsten die Probanden der EG 2 mit 5.9 (Tabelle 2). Die Ergebnisse der drei Gruppen unterscheiden sich jedoch nicht signifikant voneinander $(n=101$; Kruskal-Wallis-Test $p=.343, \alpha=.05)$. Vor dem Hintergrund, dass die Probanden der KG keine Webseiten exploriert haben, erreichen sie einen vergleichsweise hohen Mittelwert.

Tab. 2: Ergebnisse des Wissenstests

\begin{tabular}{llllll}
\hline & $n$ & $M$ & Min & Max & SD \\
\hline EG 1 & 34 & 5.5 & 1 & 10 & 2 \\
EG 2 & 31 & 5.9 & 2 & 11 & 2 \\
KG & 36 & 5.1 & 1 & 9 & 2 \\
\hline
\end{tabular}

Quelle: Eigene Darstellung

Vergleicht man die Selbsteinschätzungen der bisherigen Erfahrungen mit verschiedenen Formen von Geldanlagen und Finanzierungen (Faktor 1), des Finanzwissens (Faktor 2) sowie der subjektiven Bedeutung von Wohneigentum (Faktor 3) zeigt sich, dass die Probanden der KG über signifikant mehr Finanzerfahrungen (Faktor 1) als die Probanden der beiden EG $(n=99$, Kruskal-Wallis-Test $d f=2, \chi^{2}=11.865, p=.003$ ) verfügen. Im Hinblick auf die anderen beiden Faktoren zeigen sich keine signifikanten Unterschiede. Eine mögliche Begründung für die unterschiedlichen Finanzerfahrungen kann darin liegen, dass ein Drittel der Probanden der KG, aber nur 12\% der EG 1 und 23\% der EG 2 über eine abgeschlossene kaufmännische Berufsausbildung verfügen. Eine Korrelationsanalyse zeigt, dass die kaufmännische Ausbildung einen signifikant positiven Zusammenhang sowohl mit der Finanzerfahrung ( $n=98$, Spearman's $\rho=.314, p=.002$, 2-seitig) als auch mit dem Finanzwissen aufweist $(\rho=.311, p=.002)$.

Um mögliche Zusammenhänge zwischen den drei Faktoren und den Ergebnissen des Wissenstests zu prüfen, wurden weitere Korrelationsanalysen durchgeführt. Um die Wissenstests um zufällig richtig markierte Antworten 
zu bereinigen, wurde der jeweils erreichte Gesamtwert um den Erwartungswert reduziert. Von den Faktoren zeigen sowohl das selbsteingeschätzte Finanzwissen (Faktor 2; $n=99$, Pearson's $r=.246, p=.007$, einseitig) als auch die Finanzerfahrung $(r=.171, p=.045)$ einen signifikant positiven Zusammenhang mit den Ergebnissen des Wissenstests (abgzl. Erwartungswert), nicht jedoch die subjektive Bedeutung von Wohneigentum (Faktor 3; $r=.092, p=.183$ ).

Hinsichtlich der ersten komplexen Entscheidungsaufgabe sind die Anteile der Personen, die sich für (1) bzw. gegen (0) die Aufnahme des Baufinanzierungsdarlehens entscheiden, in beiden Experimentalgruppen vergleichbar (Tabelle 3). Nahezu zwei Drittel der Probanden votieren gegen eine Finanzierung, handeln damit also eher risikoavers. Bei der zweiten Entscheidungsaufgabe votieren 88.2 Prozent der EG 1 und 93.5 Prozent der EG 2 gegen eine Finanzierung.

\section{Tab. 3: Entscheidungen der Probanden}

\begin{tabular}{ccc|cc|ccc|cc}
\hline \multicolumn{4}{c|}{ Entscheidungsaufgabe 1 (EA 1) } & \multicolumn{3}{c}{ Entscheidungsaufgabe 2 (EA 2) } \\
& \multicolumn{2}{c}{0} & \multicolumn{2}{c}{1} & \multicolumn{2}{c}{0} & \multicolumn{2}{c}{1} \\
& $H$ & $h$ & $H$ & $h$ & $H$ & $h$ & $H$ & $h$ \\
\hline EG 1 & 21 & $61.8 \%$ & 13 & $38.2 \%$ & 30 & $88.2 \%$ & 4 & $11.8 \%$ \\
EG 2 & 19 & $61.3 \%$ & 12 & $38.7 \%$ & 29 & $93.5 \%$ & 2 & $6.5 \%$ \\
\hline
\end{tabular}

0 = Entscheidung gegen Darlehensaufnahme, 1 = Entscheidung für Darlehensaufnahme

Quelle: Eigene Darstellung

Im nächsten Schritt wurden die angeführten Argumente inhaltlich analysiert und mit der Referenzargumentation verglichen. Die Tabellen 4 und 5 geben einen Überblick über die wesentlichen Referenzargumente sowie die häufigsten Probandenargumente gegen bzw. für eine Finanzierung. Von den am häufigsten genannten finden sich in den Argumenten der Probanden für EA 1 nur zwei Argumente der Referenzargumentation gegen eine Finanzierung (Laufzeit zu lang, anfänglicher Tilgungssatz zu niedrig) wieder (Tabelle 4). Bei den Argumenten für eine Finanzierung wird im Vergleich zur Referenzargumentation nur ein übereinstimmendes Argument (Rate kleiner als frei verfügbares Einkommen) genannt (Tabelle 5). 
Tab. 4: Häufigste Argumente gegen eine Finanzierung für EA 1

\begin{tabular}{|c|c|c|c|}
\hline Argumente & $\begin{array}{l}\text { Referenz- } \\
\text { argumente }\end{array}$ & $\begin{array}{c}\text { EG } 1 \\
\text { (H Nen- } \\
\text { nungen) }\end{array}$ & $\begin{array}{c}\text { EG } 2 \\
\text { (H Nen- } \\
\text { nungen) }\end{array}$ \\
\hline Laufzeit zu lang & $\mathrm{x}$ & 17 & 11 \\
\hline anfänglicher Tilgungssatz zu niedrig & $\mathrm{x}$ & & 4 \\
\hline Restschuld ist $\mathrm{zu}$ hoch & & 11 & 4 \\
\hline frei verfügbares Einkommen niedrig & & 8 & \\
\hline $\begin{array}{l}\text { Ausgaben für Unvorhergesehenes } \\
\text { berücksichtigen }\end{array}$ & & 6 & \\
\hline Sollzinsbindung zu kurz & $\mathrm{x}$ & & \\
\hline Instandhaltungskosten beachten & $\mathrm{x}$ & & \\
\hline $\begin{array}{l}\text { Zins nach Zinsfestschreibung unbe- } \\
\text { kannt }\end{array}$ & $x$ & & \\
\hline
\end{tabular}

Quelle: Eigene Darstellung

Tab. 5: Häufigste Argumente für eine Finanzierung für EA 1

\begin{tabular}{lccc}
\hline Argumente & $\begin{array}{c}\text { Referenz- } \\
\text { argumente }\end{array}$ & $\begin{array}{c}\text { EG 1 } \\
\text { (H Nen- } \\
\text { nungen })\end{array}$ & $\begin{array}{c}\text { EG 2 } \\
\text { (H Nen- } \\
\text { nungen })\end{array}$ \\
\hline $\begin{array}{l}\text { Rate kleiner als frei verfügbares Ein- } \\
\text { kommen }\end{array}$ & $\mathrm{x}$ & 10 & 9 \\
\hline Zins niedrig & $\mathrm{x}$ & $\mathrm{x}$ \\
\hline Laufzeit angemessen & $\mathrm{x}$ & \\
\hline
\end{tabular}

Quelle: Eigene Darstellung

Auffällig in den Argumenten zur EA 2 (Tabelle 6) ist die Vielzahl der von den Probanden genannten, aber nicht in der Referenzargumentation enthaltenen Argumente. Zwar wird von 13 Probanden der EG 2 genannt, dass durch den Zinsanstieg die Annuität größer wird, aber nur 8 Probanden führen als 
Argument gegen eine Finanzierung an, dass die Rate das frei verfügbare Einkommen übersteigt. Bedenklich sind das inhaltlich verkürzte Argument und damit ein nicht korrekt dargestellter Zusammenhang in Form von ,Zinsanstieg verringert Darlehenslaufzeit". Grundsätzlich würde ein höherer Zins bei gleichbleibender Annuität i. d. R. zu einer Verlängerung der Laufzeit führen. Eine höhere Annuität bei höherem Zinsniveau führt aber dazu, dass mit fortschreitendem Darlehensverlauf der dann höhere Tilgungsanteil an der Annuität die Darlehenslaufzeit verkürzen kann. Da im Zuge der Exploration der Webseiten - abhängig von der Funktionalität der angebotenen Kalkulatoren - nicht alle Größen durch die Nutzer/innen manipulierbar waren (beispielsweise der Betrag der Annuität nicht fixiert werden kann), sind derartige Zusammenhänge jedoch ggf. schwer zu entdecken. Diese Beschränkungen auf den Webseiten können dazu führen, dass die Nutzer/innen nicht korrekte Vorstellungen zu den Wirkungszusammenhängen der Annuitätenrechnung entwickeln.

Tab. 6: Häufigste Argumente gegen eine Finanzierung für EA 2

\begin{tabular}{|c|c|c|c|}
\hline Argumente & $\begin{array}{l}\text { Refe- } \\
\text { renzar- } \\
\text { gumente }\end{array}$ & $\begin{array}{c}\text { EG 1 } \\
\text { ( } H \text { Nen- } \\
\text { nungen) }\end{array}$ & $\begin{array}{c}\text { EG } 2 \\
\text { ( } H \text { Nen- } \\
\text { nungen) }\end{array}$ \\
\hline $\begin{array}{l}\text { Rate größer als frei verfügbares Einkom- } \\
\text { men }\end{array}$ & $\mathrm{x}$ & 19 & 8 \\
\hline Restschuld zu hoch & $\mathrm{x}$ & & \\
\hline Zins nach Zinsfestschreibung unbekannt & $\mathrm{x}$ & & \\
\hline durch Zinsanstieg wird Annuität größer & & 16 & 13 \\
\hline Zinsanstieg verlängert Darlehenslaufzeit & & & 10 \\
\hline frei verfügbares Einkommen niedrig & & & 7 \\
\hline Zinsanstieg verringert Darlehenslaufzeit & & 16 & \\
\hline Zinssatz zu hoch & & 7 & \\
\hline
\end{tabular}

Quelle: Eigene Darstellung

Quantifiziert man die Unterschiede zwischen der Probandenargumentation und der Referenzargumentation auf Basis der Distanzmaße zeigt sich Folgendes: Für EA 1 und die EG 1 liegt der Mittelwert der Distanz zur Refe- 
renzargumentation bei $0.86(S D=0.1)$, für die EG 2 bei $0.88(S D=0.1)$. Da die minimale Distanz bei 0 (vollkommene Übereinstimmung mit der Referenzargumentation) und die höchste Distanz bei 1 (keine Übereinstimmung mit der Referenzargumentation) liegen, sind die Distanzwerte insgesamt als hoch einzuschätzen. Dementsprechend bestehen kaum Übereinstimmungen mit der Referenzargumentation. Für EA 2 zeigen sich für die EG 2 höhere Distanzen zur Referenzargumentation als für EG 1. Der Mittelwert der Distanz zur Referenzargumentation erreicht für EG $10.88(S D=0.1)$ und für EG $20.94(S D=0.1)$. Für beide Entscheidungsaufgaben unterscheiden sich die EG nicht signifikant in den Distanzen zur Referenzargumentation (E1: $n=65$, Kruskal-Wallis, $d f=3, \chi^{2}=1.725, p=.189$; E2: $n=65$, KruskalWallis $\left.\chi^{2}=3.187, p=.074\right)$.

Auf Basis dieser Daten kann nicht davon ausgegangen werden, dass die Darlehenskalkulatoren die Probanden in der Entwicklung von Entscheidungsfähigkeit unterstützen. Obwohl die Kalkulatoren unterschiedlich sind, wirken sich diese Unterschiede nicht auf die Fähigkeit, Entscheidungen zu begründen aus.

\section{Diskussion und Ausblick}

Die Probanden der beiden EG, die Webseiten exploriert haben, schneiden im Wissenstest tendenziell besser ab als die KG. Der Unterschied ist jedoch nicht signifikant. Hinsichtlich der aus den Selbsteinschätzungen bisherigen Finanzwissens und -erfahrungen extrahierten Faktoren zeigen das Finanzwissen und die Finanzerfahrungen signifikant positive Zusammenhänge mit dem Wissen über Baufinanzierung. Allerdings verfügt die $\mathrm{KG}$ über signifikant mehr Finanzerfahrung, was möglicherweise darauf zurückgeführt werden kann, dass eine vergleichsweise hohe Anzahl von Probanden der KG eine kaufmännische Ausbildung abgeschlossen hat. Hinsichtlich der Bedeutung von Wohneigentum unterscheiden sich die Gruppen nicht signifikant, und ein Zusammenhangmit dem Wissen ist nicht nachweisbar. Insgesamt kann vermutet werden, dass Vorwissen und -erfahrung den Wissenserwerb entscheidend beeinflussen.

Unter der Annahme, dass sich Finanzkompetenz in Bezug auf Baufinanzierungen insbesondere in den Argumenten ausdrückt, die in Entscheidungssituationen angeführt werden, zeigen sich insgesamt keine zufriedenstellenden Ausprägungen. Die wesentlichen Argumente für bzw. gegen die Aufnahme einer Baufinanzierung werden von den Probanden nur zum Teil angeführt. Die Argumentationen in beiden Gruppen stimmen nur in geringem 
$\mathrm{Ma} ß$ mit der Referenzargumentation überein, was durch die hohen Distanzwerte indiziert wird. Als problematisch sind insbesondere Argumente zu sehen, die darauf hinweisen, dass Zusammenhänge nicht durchdrungen und verstanden wurden, wie das oben ausgeführte Beispiel von Annuität und Darlehenslaufzeit zeigt. $\mathrm{Zu}$ vermuten ist, dass begrenzte Funktionalitäten der Kalkulatoren kein umfassendes Verständnis ermöglichen.

Die Probanden der EG 1 (NordLB) erzielen etwas bessere Werte als die Probanden der EG 2 (CoBa) in Bezug auf die EA 1. In Bezug auf die EA 2 ist dieses Verhältnis umgekehrt. Interessant ist dieses Ergebnis vor dem Hintergrund des geringen Umfangs an Informationen und fehlender Erklärungen auf der Webseite der NordLB. Im Vergleich dazu bietet die CoBa viele Konzepte und Erklärungen. Die Menge der Informationen scheint sich jedoch nicht positiv auf das Lernergebnis auszuwirken. Vorsichtig geschlussfolgert scheint bei geringen Vorerfahrungen tendenziell eine vergleichsweise große Menge an Informationen $(\mathrm{CoBa})$ den Lernprozess nicht besser zu unterstützen.

Bezogen auf die eingangs genannten Kompetenzfacetten lässt sich insgesamt feststellen, dass die Probanden mithilfe der Webseiten weder genügend Wissen erwerben noch zufriedenstellend in der Lage sind, dieses Wissen in Entscheidungssituationen anzuwenden. Das erreichte Niveau der Finanzkompetenz in Bezug auf Entscheidungen über Baufinanzierung kann eher als niedrig eingeschätzt werden. Vorwissen und Vorerfahrungen scheinen bedeutsamer zu sein als der Inhalt der Webseiten. Insofern lässt sich auf Basis der vorliegenden Daten kein Rückschluss auf den optimalen Inhalt von Webseiten ziehen.

Grenzen der Studie sehen wir vor allem darin, dass die KG nach eigener Einschätzung über signifikant mehr Finanzerfahrung verfügt als die Probanden der beiden EG. Dadurch wird die Zulässigkeit eines Gruppenvergleichs zumindest fraglich. Derzeit lässt sich nicht sagen, inwieweit dadurch der Vergleich ausgeschlossen ist. In einer Folgestudie sollte die Vergleichbarkeit der Gruppen im Vorfeld sichergestellt werden. Werden die sozioökonomischen Daten wie Alter und kaufmännische Ausbildung im Vorfeld erhoben, kann eine Aufteilung auf die Gruppen gesteuert werden. Ein weiterer Punkt ist, dass die Kontrollgruppe keine Entscheidungsaufgaben bearbeitet hat. Dies lag darin begründet, dass in unserer Studie die Entscheidungsaufgaben unter Hinzuziehung der Webseiten bearbeitet werden sollten, die Kontrollgruppe jedoch keinen Zugang zu den Informationen der Webseiten haben sollte. In einer Folgestudie könnten jedoch die Entscheidungsaufgaben auch durch die Kontrollgruppe bearbeitet werden, um so weitere Rückschlüsse auf die Leistungsfähigkeit der Webseiten zur Unterstützung einer begründeten Entscheidungsfindung ziehen zu können. Ein weiterer Punkt ist, die Disposition der 
Risikofreude bzw. Risikoaversion der Probanden systematisch als Kontrollvariable einzubeziehen und nicht nur aus den Entscheidungen für oder gegen eine Darlehensaufnahme indirekt zu erschließen.

Neben den angesprochenen Punkten ist für weitere Studien zu überlegen, reale Webseiten im Vergleich zu einer speziell konstruierten Webseite (entsprechend dem Referenzkalkulator) in einer experimentellen Studie zu untersuchen, um genaueren Aufschluss über die Einflussfaktoren (z. B. Quantität und Qualität von Informationen) auf die Kompetenzentwicklung zu gewinnen. Ferner können weitere Faktoren einbezogen werden, die sich auf die Entwicklung von Finanzkompetenz auswirken bzw. erzielte Ergebnisse erklären können. Hierzu gehören z. B. das multimediale Design der Webseiten, die Art und Weise der kognitiven Informationsverarbeitung oder auch kognitive und metakognitive Strategien der Probanden.

\section{Danksagung}

Wir danken den beiden anonymen Reviewern für ihre hilfreichen Hinweise zu diesem Artikel.

\section{Literatur}

Aprea, C., Wuttke, E., Breuer, K., Keng Koh, N., Davies, P., Greimel-Fuhrmann, B. \& Lopus, J.S. (2016). Financial literacy in the twenty-first century. An introduction to the international handbook of financial literacy. In: C. Aprea, E. Wuttke, K. Breuer, N. Keng Koh, P. Davies, B. Greimel-Fuhrmann \& J. S. Lopus (Hrsg.). (2016). International Handbook of Financial Literacy (S. 1-8). Singapore: Springer.

Fürstenau, B. \& Trojahner, I. (2005). Prototypische Netzwerke als Ergebnis struktureller Inhaltsanalysen. In P. Gonon, F. Klauser, R. Nickolaus \& R. Huisinga (Hrsg.), Kompetenz, Kognition und neue Konzepte der beruflichen Bildung (S. 191-202), Opladen: Leske + Budrich.

Fürstenau, B., Hommel, M., Leopold, C., Ponce, H. \& López, M. (2015). Baufinanzierung nach Maß? - Aufbau von Finanzkompetenz durch Online-Informationen. In J. Seifried, S. Seeber \& B. Ziegler (Hrsg.) Jahrbuch der berufs- und wirtschaftspädagogischen Forschung 2015. Schriftenreihe der Sektion Berufs- und Wirtschaftspädagogik der Deutschen Gesellschaft für Erziehungswissenschaft (DGfE) (S. 23-36). Opladen, Berlin, Toronto: Barbara Budrich. 
Fürstenau, B., Hommel, M., Leopold, C., Ponce, H. \& López, M. (2016). Analysis of banks' online information regarding mortgages as a basis for financially literate decision-making in first-time homebying. In. E. Wuttke, J. Seifried \& S. Schumann, S. (Eds.). Economic Competence and Financial Literacy of Young Adults Status and Challenges (S. 63-80). Opladen, Berlin, Toronto: Barbara Budrich.

Gerardi, K., Goette, L. \& Meier, S. (2010). Financial literacy and subprime mortgage delinquency: Evidence from a survey matched to administrative data. Working Paper, Federal Reserve Bank of Atlanta, No. 2010-10, [online] http://www. econstor.eu/bitstream/10419/70651/1/637330404.pdf. (20.03.2017)

GSA General Services Administration, Federal Citizen Information Center (2016). Consumer Action Handbook. Online: https://publications.usa.gov/USAPubs.php? PubID=5131 (20.03.2017).

OECD (2013). PISA 2012 Assessment and analytical framework: Mathematics, reading, science, problem solving and financial literacy. OECD Publishing. Online: https://www.oecd.org/pisa/pisaproducts/PISA\%202012\%20framework\%20ebook_final.pdf (20.03.2017).

Mayring, P. (2015). Qualitative Inhaltsanalyse. Grundlagen und Techniken. (12. Aufl.). Weinheim u. a.: Beltz.

Roediger, H. L. \& Karpicke, J. D. (2006). Test-enhanced learning: Taking memory tests improves long-term retention. Psychological Science. 17 (3): 249-255.

Seeber, S. (2016). Vom Domänenmodell zum Kompetenzmodell: Konturen eines Assessmentdesigns zur Messung beruflicher Fachkompetenzen bei Medizinischen Fachangestellten. In bwp@Berufs- und Wirtschaftspädagogik online, Profil 4: Kompetenzentwicklung im wirtschaftspädagogischen Kontext: Programmatik - Modellierung - Analyse. Digitale Festschrift für Sabine Matthäus, 1-25. Online: http://www.bwpat.de/profil4/seeber_profil4.pdf (24.11.2016).

Shavelson, R. J. (2010). On the measurement of competency. Empirical Research in Vocational Education and Training, 2(1), S. 41-63.

Shavelson, R. J. (2013). On an approach to testing and modeling competence. Educational Psychologist, 48(2), 73-86. 



\title{
Zur Ausgestaltung schulischer Praxisphasen im beruflichen Lehramtsstudium - Ergebnisse einer ersten Analyse
}

\author{
Silke Lange und Dietmar Frommberger
}

\section{Einleitung}

In der universitären Lehramtsausbildung in Deutschland sind schulische Praxisphasen etabliert. Umfang, Rahmenbedingungen und Ausgestaltung dieser werden unter dem Stichwort „Praxisbezug“ vielfach und kontrovers diskutiert. Insbesondere durch die Einführung von Bachelor- und Masterstrukturen wurde die Debatte um die schulische Praxis im Studium wieder vermehrt in den Fokus gerückt (vgl. Gröschner et al. 2013; Weyland 2014). Der Wert der schulpraktischen Phasen wird dabei per se als unbestreitbar aufgefasst (vgl. Hascher 2011).

Bisher liegen jedoch nur wenige belastbare Befunde über die Wirksamkeit und erfolgswirksamen Rahmenbedingungen schulpraktischer Studien vor. Zwar hat die empirische Erforschung der schulpraktischen Ausbildungsanteile im Lehramtsstudium in den letzten Jahren deutlich zugenommen, ${ }^{1}$ dennoch ist die Reichweite der Untersuchungsergebnisse häufig auf einzelne Standorte beschränkt und es liegen nur ansatzweise standortübergreifende Befunde vor (vgl. Gröschner et al. 2015).

Den Herausforderungen im Zusammenhang mit der Professionalisierung der Lehramtsstudierenden begegnen die universitären Lehrerbildungsstandorte in Deutschland mit unterschiedlichen Konzeptionen schulpraktischer Ausbildungsanteile, was sich auch auf die mangelnde Erkenntnislage zu schulpraktischer Studienanteilen und deren Gestaltung zurückführen lässt (vgl. Lange i. E.). Verschiedene Expertisen und Übersichten geben einen allgemeinen, alle Lehramtstypen umfassenden Überblick über den Stand der Reformen in der schulpraktischen Ausbildung des Lehramtsstudiums in Deutschland (vgl. u. a. Gröschner et al. 2015; Offenberg et al. 2013; Weyland 2012). Eine Übersicht über die aktuellen Schulpraxisphasenmodelle im beruflichen Lehramtsstudium liegt bisher nicht vor - obwohl die berufliche Bildung und das berufliche Lehramtsstudium durch Besonderheiten geprägt sind (vgl. u. a. Becker, Spöttl 2013). Inwieweit diese Besonderheiten des

1 Einen Überblick über das mittlerweile florierende Forschungsfeld geben u. a. Besa \& Büdcher (2014), Hascher (2012a, 2012b) sowie Weyland (2014). 
beruflichen Lehramts(-studiums) sich in diesen Konzepten widerspiegeln, lässt sich derzeit nicht erörtern. Auch ist nicht klar, welche Gestaltungsmöglichkeiten und Rahmenbedingungen das Lernen in schulpraktischen Studienphasen umrahmen und unterstützten.

Zur Beantwortung dieser Fragerichtungen sind zunächst Untersuchungen notwendig, die sich auf strukturelle Aspekte beziehen und grundlegende Konzepte der schulpraktischen Ausbildung in der ersten Phase identifizieren (vgl. Gröschner et al. 2015, 646). Eine solche Untersuchung wird im vorliegenden Beitrag vorgestellt. Ziel der Untersuchung ist es, die an den einzelnen Standorten des beruflichen Lehramtsstudiums implementierten Schulpraxiselemente anhand verschiedener Merkmale näher zu beschreiben. Damit schließt die Untersuchung an vorliegende Expertisen an und erweitert den Diskurs um das berufliche Lehramtstudium. Basierend auf einer Fragebogenerhebung wird den Fragen nachgegangen, wie schulische Praxisphasen im so genannten Lehramtstyp 5 strukturiert sind, wie die universitäre Begleitung dieser organisiert ist, in welcher Form diese Ausbildungsanteile curricular in das Studium integriert sind und inwiefern sich auf dieser Basis standortübergreifende Praxistypen identifizieren lassen.

\section{Zur Kontroverse der schulpraktischen Ausbildungsanteile im Lehramtsstudium}

Die Schulpraxis war und ist das ,wesentliche konstitutive Ausbildungselement" (Bennack, Jürgens 2002, 143) der Lehrerausbildung. Seit der Akademisierung der Lehramtsausbildung wird über Form und Inhalt derselben diskutiert (vgl. Helsper et al. 2002). „Der Ruf nach ,Mehr Praxis!' war und ist" dabei ,eines der stabilsten Elemente im älteren wie neueren Reformdiskurs" (Terhart 2013, 5). Gleichzeitig wird die häufig marginale und isolierte Stellung der schulpraktischen Studien kritisiert, die diese zum „Stiefkind innerhalb der Lehramtsstudiengänge“ (Glumper \& Wildt 2000, zit. n. Weyland 2010,6) werden ließ. Dies spiegelt sich auch im Erkenntnisstand zu den schulpraktischen Ausbildungsanteilen im Lehramtsstudium wider. Erst in den letzten Jahren hat die Erforschung der Wirksamkeit schulischer Praxisphasen zugenommen, nachdem „die Qualität berufspraktischer Ausbildung lange Zeit unhinterfragt blieb" (Hascher 2011, 12). Der Blick auf die empirische Befundlage zur Wirksamkeit schulpraktischer Studien rät zur Bescheidenheit, "was die Erwartungen an das Lernpotential von Praktika [...] anbelangt" (Hascher 2006, 145). Die vorliegenden Ergebnisse zeigen, dass sich in Praktika sowohl erwünschte als auch unerwünschte Entwicklungen vollziehen, und offerieren vielseitige Prolemlagen (Hascher 2006, 2012a). 
Die Begriffe Praxis, Praxisbezug, schulpraktische Ausbildung etc. werden dabei sowohl in der Literatur als auch im Alltagsbewusstsein nicht einheitlich verwendet (vgl. Schubarth et al. 2012a, 52). Die Formen von Praxisbezügen differenzieren in den Studiengängen: Sie reichen von forschungsorientierten Zugängen, in denen die Praxis zum Forschungsgegenstand wird (Praxisforschung), über lehr- und dialogorientierte Zugänge, bei denen die Praxis als Thema der Lehre bzw. des Dialogs aufgenommen wird, bis hin zu den eigentlichen Praktika, in denen neben dem Studium Arbeitserfahrungen an zukünftigen Arbeitsorten gesammelt werden (vgl. Bargel 2012, 37; Schubarth et al. 2012a, 52). Die schulpraktischen Studien, wie sie von der KMK $(2005,3)$ bereits für das Bachelorstudium gefordert werden, werden i. d. R. als Praktika im eigentlichen Sinn, also Erfahrungsraum am zukünftigen Arbeitsort berufsbildende bzw. berufliche Schulen, aufgefasst und untersucht. Für die Bezeichnung dieser Ausbildungsanteile werden verschiedene Termini genutzt, die jedoch vielfach einen Bezug zum Praktikum und zum Lernort Schule ausdrücken (vgl. Lange i. E.). Darunter fallen bspw. Termini wie schulische Praxisphasen, schulische Praxis, schulische Studien, schulpraktische Ausbildung, Schulpraxis, Praktikum etc., die im Beitrag synonym verwendet werden.

Die vorliegenden Befunde zu den schulpraktischen Ausbildungsanteilen im Studium sind insgesamt sehr heterogen: Es zeigt sich, dass die Praxisphasenmodelle eng an die an der Hochschule jeweils vorherrschenden Lehrerbildungsmodelle geknüpft sind, die zwischen und auch innerhalb der Bundesländer variieren (vgl. zusammenfassend Gröschner et al. 2015, 642). Dabei verfolgen die schulpraktischen Studien unterschiedliche Zielsetzungen, die u. a. durch die Platzierung im Studienverlauf (vgl. Gröschner et al. 2015, 643) und unterschiedliche Organisationsformen (vgl Schubarth et al. 2012b, 138) erreicht werden sollen. Bisher fehlen jedoch weitergehende empirische Studien, die die Platzierung im Studienverlauf und die Organisationsform von schulischen Praktika insbesondere im Hinblick auf deren Wirksamkeit in den Blick nehmen (vgl. Gröschner et al. 2015, 644). Erste Befunde zeugen jedoch davon, dass die Studierenden im Praktikum oftmals von einer Lernsituation zur nächsten springen, ohne dass der Lernprozess systematisch gestaltet wird (vgl. Hascher 2012a, 122). Deshalb wird der Vor- und Nachbereitung sowie der Begleitung und Betreuung der Praktikumsphasen eine besondere Bedeutung zugeschrieben (vgl. Hascher 2012a, 123; Schubarth et al. 2012a, $82 ; 2012 b, 138$ ), auch um die Aneignung unhinterfragter Praxis zu vermeiden (vgl. Hascher 2012a, 122; Holtz et al. 2013). Zur Überwindung des Problems ist die Einbeziehung und Schulung der Betreuungspersonen unabdingbar (vgl. Holtz et al. 2013). Untersuchungen, die auf die Wirksamkeit von Praxisphasen fokussieren, weisen zum Teil einen Kompetenzzuwachs der Studierenden nach, andere Untersuchungen können jedoch kaum Kompetenzverän- 
derungen verzeichnen. ${ }^{2}$,Allerdings ist die Wirksamkeit bei weitem noch nicht hinreichend überprüft" (Hascher 2012a, 123).

Trotz der heterogenen Befundlage besteht die Grundüberzeugung, dass Praxisphasen für die Professionalisierung wichtig und notwendig sind. Insofern ist es nicht verwunderlich, dass über alle Lehramtstypen hinweg aktuell ein Trend zur Ausweitung der schulischen Praxisphasen zu verzeichnen ist (vgl. Offenberg, Walke 2013; Weyland 2012). Erkennbar ist dabei ein „bundeslandübergreifendes Bestreben zur Einrichtung von Praxissemestern“ (Weyland 2012, 4), das zum Teil von einer Verkürzung des Vorbereitungsdienstes begleitet wird (vgl. Weyland 2014).

Pointiert zeigt sich hier die Kontroverse der schulpraktischen Ausbildungsanteile im Lehramtsstudium - Hascher (2011) spricht vom „Mythos Praktikum“. Auf der einen Seite wird der schulpraktischen Ausbildung im Lehramtsstudium seit Jahrzehnten eine besondere Bedeutung zugeschrieben, andererseits haben schulpraktische Studien über lange Zeit keine strukturelle und curriculare Wertschätzung erhalten - die Hochschulrektorenkonferenz (HRK) sprach 1998 von einem „Randphänomen“. Zusätzlich wurde auch die Qualität der schulpraktischen Ausbildung lange Zeit nicht hinterfragt, sodass bisher nur ein spärliches Wissen über die Wirksamkeit der schulischen Praxisphasen vorhanden ist - Hascher $(2011,12)$ bezeichnet die Theorieentwicklung als „rudimentär“. Bisher lassen sich keine ,validen Hinweise auf den optimalen Zuschnitt von Praxiselementen im Lehramtsstudium“ finden (Offenberg, Walke 2013, 40). Gröschner und Kollegen (2015) führen das vor allem darauf zurück, dass bisher nur wenige Untersuchungen vorliegen, die die strukturellen Rahmenbedingungen gezielt berücksichtigen und vergleichende Analysen vornehmen. „Zumeist liegt in den auf Praxisphasen bezogenen Wirksamkeitsuntersuchungen ein starker Fokus auf einzelnen Hochschulstandorten“" (ebd., 641).

Gleichzeitig haben viele Ausbildungsstandorte in Deutschland im Rahmen von Um- und Neustrukturierungen eine Ausweitung der schulpraktischen Ausbildungsbestandteile vorgenommen. Ergebnis der Entwicklungen sind verschiedene Modelle und Ansätze im Bundesgebiet, weshalb Weyland $(2012,50)$ von einem „bundesweiten Flickenteppich“" spricht.

Um die Theorieentwicklung zum Lernen in schulpraktischen Studien voran treiben zu können, ist es nach Gröschner und Kollegen (2015) jedoch unabdingbar, standortübergreifende und damit Strukturen berücksichtigende Analysen durchzuführen. Der Bedarf an empirischer Forschung zum aktuellen Stand der Reformen sowie darauf aufbauender standortübergreifender Forschung zur wirksamen Ausgestaltung schulpraktischer Ausbildungsanteile wird hier deutlich.

2 Dies lässt sich auch auf die Anlage der Untersuchungen zurückführen. Ein Teil der Untersuchungen stellen Wirksamkeitsstudien i.e.S. dar, andere arbeiten mit Kompetenzselbsteinschätzungen, um die Kompetenzzuwächse nachzuzeichnen (vgl Hascher 2012a, 123). 


\section{Vorstellung der Analyse und der Ergebnisse}

\subsection{Untersuchungsansatz}

Vergleichbare Untersuchungen basieren auf Analysen studiengangsrelevanter Dokumente (vgl. Gröschner et al. 2015; Weyland, Wittmann 2010, Weyland 2012). Im Zuge dieser Untersuchung erfolgte eine Fragebogen-Erhebung, um eine Beschreibung der schulpraktischen universitären Ausbildungsanteile zu erhalten und Schulpraxisphasenkonzepte identifizieren zu können. Einbezogen wurden dabei nur Schulpraktika. Die für das berufliche Lehramt darüber hinaus notwendige fachpraktische Tätigkeit (vgl. KMK 2016) wurde explizit nicht einbezogen. Der Fragebogen wurde vor Beginn der Untersuchung durch KollegInnen verschiedener Bundesländer getestet und kommentiert, um der Herausforderung der Validität einzelner Fragen/Items zu begegnen. Für die Erhebung wurden 62 Personen an den 51 ausbildenden Standorten in Deutschland per E-Mail angeschrieben und um Teilnahme gebeten. Bis zum 05. Juli 2016 lagen 38 Rückmeldungen von 36 Standorten vor, das entspricht einem Anteil von 70,59\%. An der Erhebung beteiligt haben sich Standorte aus 13 der 15 ausbildenden Bundesländer, Daten fehlen derzeit aus Bremen und aus Sachsen.

\subsection{Rahmen der Untersuchung}

Schulpraxisphasen lassen sich durch eine Vielzahl unterschiedlicher Merkmale charakterisieren (vgl. Lange i. E.). Ein Konsens über die zur Beschreibung und zum Vergleich heranzuziehenden Indikatoren zeichnet sich bisher nicht ab. Aufgrund der Heterogenität der Studiengangsstrukturen ist jedoch eine Fokussierung auf ausgewählte Aspekte unabdingbar. In der vorliegenden Untersuchung wurden vor dem Hintergrund der forschungsleitenden Fragestellungen, die einleitend präzisiert wurden, die Strukturen, die Begleitung und die curriculare Einbindung der schulischen Praxisphasen fokussiert (Abbildung 1).

Auf der strukturellen Ebene können die Praktika hinsichtlich vielfältiger Merkmale unterschieden werden. Zunächst ist festzustellen, dass die Anzahl und die Bezeichnung sowie die Dauer der Praktika an den Ausbildungsstandorten variieren (vgl. u. a. Weyland 2012). Auch bezogen auf die institutionelle Zuständigkeit lassen sich divergente Entwicklungen beobachten. Einerseits werden Praktika in der Verantwortung der Hochschulen durchgeführt, andererseits setzen einige Bundesländer auf die Zuständigkeit der Staatlichen Seminare für die schulpraktische Ausbildung bereits während der universitären ersten Ausbildungsphase (vgl. ebd.). Auf der temporär- 
strukturellen Ebene können Praktikumskonzepte darüber hinaus hinsichtlich der Organisationsform - als Blockpraktika oder semesterbegleitend angelegte Praktika (vgl. Bennack, Jürgens 2002; Gröschner et al. 2015) - und der Verortung im Studienverlauf unterschieden werden.

\section{Abb. 1: Kategorienraster zur Beschreibung der schulischen Praxisphasen im beruflichen Lehramtsstudium}

\section{Strukturen schulischer Praxisphasen}

- Anzahl

- Bezeichnung

- Umfang

\section{Begleitung schulischer Praxisphasen}

- Seminaristische Begleitung

- Unterrichtsbesuche

- Mentorenqualifizierung

\section{Curriculare Einbindung schulischer Praxisphasen}

- Seminaristische Begleitung

- Leistungsnachweise

- Leistungspunkte
- Benotung

- Praktikumsübergreifendes Konzept

Quelle: Eigene Darstellung

Neben den strukturellen Rahmenbedingungen wird in vielen Untersuchungen die Begleitung schulischer Praxisphasen einbezogen, da diese als erfolgswirksames Element der schulpraktischen Ausbildung gilt (vgl. Gröschner et al. 2015; Hascher 2012a; 2012b). Vorliegende Befunde zeigen, dass die Qualität der Betreuung ein wesentlicher Einflussfaktor für das Lernen im Praktikum ist und gute Lerneffekte dann erzielt werden, wenn die Praktika systematisch durch Lehrveranstaltungen begleitet werden (vgl. ebd.). Relevante Indikatoren für die Begleitung der Schulpraktika sind daher die seminaristische Begleitung, die Unterrichtsbesuche und die Qualifizierung der schulischen Mentoren.

Auch auf der Ebene der curricularen Einbindung ist die Art der seminaristischen Begleitung zu berücksichtigen, da diese nicht nur Hinweise auf die Begleitung schulpraktischer Phasen liefert, sondern auch eine Form der curricularen Einbindung in die (Lehramts-)Studiengänge darstellt. Neben der seminaristischen Begleitung liefern die zu erwerbenden Leistungspunkte (als Maßstab für den Lernzeitaufwand der Studierenden), die dafür notwendigen Leistungsnachweise und die Benotung dieser Leistungen Hinweise auf die curriculare Einbindung. Weyland (2012) folgend wird auch die Abstimmung der einzelnen schulischen Praxisphasen untereinander zur curricularen Ein- 
bindung gezählt, weshalb praktikumsübergreifende Konzepte als Indikatoren für die curriculare Einbettung der schulischen Praxisphasen herangezogen werden. Hierzu zählt auch das Konzept des Schulpraxissemesters. Als Schulpraxissemester wird eine mehrmonatige Praxisphase verstanden, in der sich Studierende für einen vorgegebenen Zeitraum am Lernort Schule aufhalten. Vorbereitung, Begleitung und Nachbereitung dieser Phase sind durch ein entsprechendes Konzept abgestimmt (vgl. Perle 1999; BaSS 2011). Eine detaillierte curriculare Bestimmung der Schulpraxisphasen über die Inhalte und gewünschten Verhaltensdispositionen in den Modulbeschreibungen kann im Rahmen der vorliegenden Untersuchung aus forschungspraktischen Gründen nicht berücksichtigt werden.

\subsection{Darstellung und Diskussion ausgewählter Ergebnisse der Untersuchung ${ }^{3}$}

Auf der strukturellen Ebene zeigt sich, dass die Studierenden im Bachelorstudium durchschnittlich zwei Schulpraktika $(\mathrm{M}=1,87, \mathrm{SD}=1,042)$ absolvieren. Diese finden vorwiegend in Blockform statt $(88,37 \%)$ und dauern im Durchschnitt 3,78 Wochen ( $\mathrm{SD}=0,989)$. In der gesamten Bachelorphase absolvieren die Studierenden Schulpraktika im Umfang von insgesamt durchschnittlich 5,56 Wochen ( $\mathrm{SD}=2,256)$.

Im Masterstudium sind die Studierenden im Durchschnitt für ein Praktikum $(\mathrm{M}=1,38, \mathrm{SD}=0,609)$ in der Schule. Der Anteil der semesterbegleitenden Praktika ist in der Masterphase mit 29,27 \% etwas höher als in der Bachelorphase, liegt jedoch noch immer unter dem Anteil der Blockpraktika. Die Praktika weisen im Durchschnitt einen Umfang von 7,94 Wochen $(\mathrm{SD}=7,124)$ auf. Insgesamt verbringen die Studierenden in der Masterphase durchschnittlich 10,21 Praktikumswochen in der Schule, wobei die Standardabweichung mit 11,72 Wochen sehr hoch ausfällt, was sich durch die Differenz der Konzepte erklären lässt: Praxissemester sind darauf ausgelegt, dass die Studierenden über einen längeren Zeitraum an der Schule sind, andere Konzepte setzen dagegen auf mehrere und kürzere Praktikumsphasen.

Über Bachelor und Master hinweg absolvieren die Studierenden zwischen einem und sieben, im Durchschnitt drei Schulpraktika, wobei im Bachelor durchschnittlich mehr Schulpraktika vorgesehen sind, als im Masterstudium. Im Vergleich beider Studienphasen zeigt sich, dass die Studierenden im Bachelor häufiger, gleichzeitig aber auch kürzer an der Schule sind, als dies im Masterstudium der Fall ist. Insgesamt ist insbesondere bezogen auf den Um-

3 In diesem Kapitel werden ausgewählte Ergebnisse der Untersuchung vorgestellt. Für weitergehende Auswertungen sei auf Lange (i.E.) verwiesen. Aus Gründen der Lesbarkeit wird auf die durchgängige Nutzung von Bachelor- bzw. Grundstudium und Master- bzw. Hauptstudium verzichtet. Stattdessen wird überwiegend von Bachelor- und Masterstudium gesprochen, da alle Standorte, zu denen Daten vorliegen, auf eine konsekutive Ausbildung umgestellt haben. 
fang der schulpraktischen Ausbildungsanteile eine deutliche Heterogenität auf der strukturellen Ebene festzustellen. Die Diversität der Praxisphasenkonzepte wird noch deutlicher, wenn zusätzlich die Bezeichnung dieser Studienanteile (vgl. Lange i. E.) und die institutionelle Zuständigkeit betrachtet werden. Für letztere stellte Weyland (2012) fest, dass die Verantwortung fast durchweg bei den Hochschulen läge, gegenläufige Entwicklungen aber zu beobachten seien. Im beruflichen Lehramtsstudium scheinen diese gegenläufigen Entwicklungen an Fahrt aufgenommen zu haben - vier Jahre später ist festzustellen, dass insbesondere in der Masterphase die Staatlichen Seminare eine große Verantwortung in der schulpraktischen Ausbildung im Studium übernehmen. ${ }^{4}$

Die dargestellte Heterogenität der Struktur der Schulpraxisphasenkonzepte im beruflichen Lehramtsstudium schließt an vorliegende Untersuchungen an (vgl. u. a. Weyland 2010; 2012) und spiegelt deutlich die mangelnde empirische Befundlage hierzu wider. Bezogen auf den Umfang der Praxisphasen wird die Diversität der Konzepte besonders deutlich. Dies ist nicht verwunderlich, da auf der einen Seite der schulpraktischen Ausbildung eine hohe Wirksamkeit zugeschrieben wird und häufig längere Praxisphasen im bildungspolitischen Kontext gefordert werden (vgl. u. a. Terhart 2013; Weyland 2014), andererseits vorliegende empirische Befunde den Vorteil der längeren Praxisphasen nicht unterstreichen. Einzeluntersuchungen stellten „,bedenkliche Negativentwicklungen bei den Studierenden mit langen schulpraktischen Ausbildungsanteilen [fest, Anm. d. Autoren]. Es verschlechtern sich sowohl ihre Berufsmotivation als auch ihre Haltung gegenüber dem Lehrerberuf als Profession“ (Hascher 2012a, 116). Und auch die subjektive Bewertung der Praxisphasen fällt mit zunehmender Praxiserfahrung kritischer aus (vgl. ebd.).

Auch bezogen auf die Organisationsform liegen bisher wenige Forschungsbefunde vor. Erste Untersuchungen haben für das Lehramt an Gymnasien keine bzw. nur geringe Unterschiede in den wahrgenommenen Lehrerträgen von Block- und semesterbegleitenden Praktika festgestellt (vgl. Gröschner et al. 2015). Insbesondere hier könnte sich jedoch eine Besonderheit des beruflichen Lehramtes - der Turnusunterricht - niederschlagen. Vor diesem Hintergrund bieten sich lehramtsspezifische Untersuchungen an. Verdeutlicht an diesen Beispielen zeigen sich auf der strukturellen Ebene durchaus Forschungsbedarfe, deren Umsetzung durch eine standortübergreifende Herangehensweise befruchtet würde.

Auf der Ebene der Begleitung schulischer Praxisphasen wurden u. a. die seminaristische Begleitung und die Mentorenqualifizierung untersucht. Unter der seminaristischen Begleitung wurden sowohl vor- und nachbereitende als auch begleitende Lehrveranstaltungen subsummiert. Die Auswer-

4 Gefragt und ausgewertet wurde, ob jeweils Vor-, Begleit- oder Nachbereitungsseminare von den Staatlichen Seminaren gestaltet und durchgeführt werden. 
tung der Daten nach der seminaristischen Begleitung ergab, dass der Großteil der Praktika begleitet durch Seminare stattfindet. Nur 16,33\% der Praktika im Bachelor und 2,22 \% der Praktika im Master finden ohne seminaristische Begleitung statt. Für die Mehrzahl der Praktika (77,55 \% im Bachelor und $84,44 \%$ im Master) werden Vorbereitungsseminare durchgeführt. Der Anteil an Begleit- und Nachbereitungsseminaren ist etwas geringer, insbesondere im Bachelorstudium. Die seminaristische Begleitung ist für die Kompetenzentwicklung der Studierenden von besonderer Bedeutung, das zeigen mehrere Studien (vgl. u. a. Weyland 2014). Auch Studierende schätzen den Praxisbezug der Praktika höher ein, wenn diese eng mit Lehrveranstaltungen verbunden und professionell vor- und nachbereitet sind (vgl. Gröschner, Schmitt 2010).

Neben der institutionellen Begleitung ist die Betreuung in der Schule ein wesentlicher Einflussfaktor für die Wirksamkeit der schulischen Praxisphasen (vgl. Weyland 2014). Viele Hochschulen $(n=14)$ qualifizieren die MentorInnen in Weiterbildungs- und Zertifikatskursen gezielt für ihre Rolle im Schulpraktikum. Hochschulen in Berlin und Bayern geben an, ihr Angebot um Weiterbildungsstudiengänge für MentorInnen erweitert zu haben. Diese Informationen sind jedoch mit Vorsicht zu betrachten, da einige Standorte auch explizit darauf hinwiesen, dass die tatsächlichen MentorInnen an den Schulen die Angebote nicht durchlaufen haben müssen, um PraktikantInnen betreuen zu können. Ebenso viele Standorte gaben jedoch an, keine formale Qualifizierung durchzuführen. Dies ist insofern verwunderlich, da die besondere Rolle der MentorInnen im Schulpraktikum bereits empirisch nachgewiesen werden konnte (vgl. Gröschner et al. 2013; Hascher 2012a). Bach und Kollegen (2014) verwiesen auf die Vielzahl der Aufgaben und Funktionen, die die Lehrkräfte in der Rolle als MentorIn ohne eine derartige Ausbildung nicht so wahrnehmen könnten, dass ein erfolgreicher Kompetenzerwerb stattfinden könne. Dennoch ist das wissenschaftliche Interesse an den MentorInnen bisher gering.

Zuletzt sollen ausgewählte Ergebnisse auf der Ebene der curricularen Einbindung dargestellt und diskutiert werden. Neben der Präsenz in der Praktikumsschule haben die Studierenden im Praktikum oftmals weitere Aufgaben und Arbeitsaufträge zu erledigen. Dazu zählen Praktikumsberichte und Portfolios sowie Unterrichtsentwürfe, Studienaufträge und -projekte, Präsentationen sowie Gespräche mit DozentInnen und MentorInnen. Dafür bekommen die Studierenden zwischen zwei und 30 Leistungspunkte. In Abbildung 2 ist zu erkennen, dass die Spannweite der Leistungspunkte zwischen Bachelor- und Masterphase stark differenziert. Im Bachelorstudium erhalten die Studierenden im Mittel 5,88 Leistungspunkte, im Masterstudium 8,83 Leistungspunkte für Studienleistungen im Rahmen des Praktikums. 


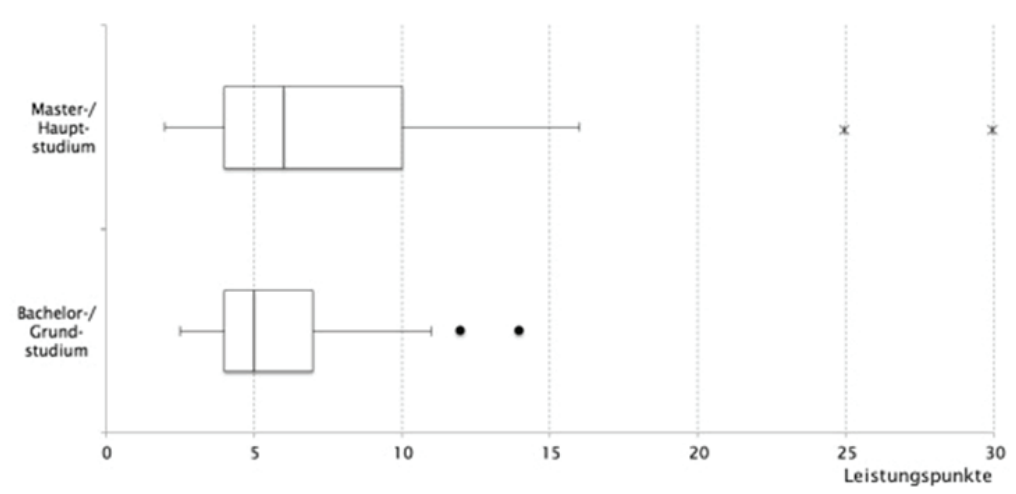

Quelle: Eigene Darstellung

Die Spannweite der vergebenen Leistungspunkte ist im Master deutlich höher als in der Bachelorphase. Der Interquartilabstand, also die Boxlänge, in der die mittleren $50 \%$ aller Werte liegen, beträgt im Bachelor drei Leistungspunkte und im Masterstudium sechs Leistungspunkte. Die Ausreißer im Bachelor liegen sehr nah am oberen Whisker, ${ }^{5}$ im Master sind Extremwerte ${ }^{6}$ zu verzeichnen, die sehr weit vom oberen Whisker entfernt liegen. Wird ausschließlich die Anzahl der vergebenen Leistungspunkte als Maß für die curriculare Wertschätzung der Praktika herangezogen, muss geschlussfolgert werden, dass die vielfach kritisierte Randständigkeit der schulpraktischen Ausbildungsanteile nicht flächendeckend vorhanden ist, sondern die curriculare Wertschätzung sehr stark differenziert. Unabhängig davon ist die Frage berechtigt, ob eine höhere curriculare Wertschätzung tatsächlich einen höheren Kompetenzerwerb nach sich zieht. Eine Frage, die auf Basis von standortbezogener Forschung sicherlich nicht zu beantworten ist, sondern standortübergreifender Forschung bedarf.

Ein weiterer Indikator für die Integration der Praktika in die theoretischen Studien ist für Weyland $(2012,5)$ die ,curriculare Abstimmung in Bezug auf die einzelnen schulischen Praxisphasen“ untereinander. In der Bachelorphase sind $43,75 \%$ der Praktika in ein praktikumsübergreifendes Konzept (einschließlich Schulpraxissemesterkonzept) eingebunden, in der

5 Als Whisker werden die von der Box abgehenden, T-förmigen Linien bezeichnet, deren Länge maximal das 1,5-fache des Interquartilabstandes, also der Boxlänge, beträgt. Der Whisker endet genau an dem Punkt, an dem sich der letzte Wert befindet, der nicht weiter als 1,5 Quartilabstände vom ersten (unteren) bzw. dritten (oberen) Quartil entfernt liegt.

6 Extremwerte sind Werte, die mehr als drei Interquartilabstände vom Median entfernt liegen. 
Masterphase ist der Anteil mit 56,25\% etwas höher. ${ }^{7}$ Nur zehn Standorte gaben an, weder im Bachelor noch im Master ein praktikumsübergreifendes Konzept implementiert zu haben. Vorreiter der praktikumsübergreifenden Konzepte ist im Masterstudium das Schulpraxissemester, welches an 35,38 \% der Standorte eingeführt ist. Welchen Mehrwert die praktikumsübergreifenden Konzepte, allen voran das Schulpraxissemesterkonzept, in der Ausbildung des beruflichen Lehramtes mit sich bringen, müssen künftige Untersuchungen zeigen. In Nordrhein-Westfalen resümierten Rexing und Klitze (2015) beispielswiese durchaus heterogene organisatorische Probleme und Herausforderungen des Schulpraxissemesters, wie beispielsweise die Differenzen zwischen Schuljahr und Semester sowie Vermittlungs- und Zuteilungsprobleme, die durch die Vielfalt des Angebotes an berufsbildenden Schulen verstärkt werden.

Im letzten Schritt der Auswertung erscheint es zunächst sinnvoll, die festgestellte Komplexität der schulpraktischen Ausbildungsmodelle durch Typenbildung zu reduzieren. Eine Typologisierung der Modelle erleichtert die Vergleichbarkeit der Standorte, was nach Weyland (2012) einerseits positive Auswirkungen auf die Mobilität der Studierenden haben kann und nach Gröschner und Kollegen (2015) andererseits die Wirksamkeitsforschung bereichern kann, da Evaluationen standortübergreifend mit typologisch ähnlichen Referenzmodellen durchgeführt werden können.

Zur Bildung der Typen in Anlehnung an Gröschner und Kollegen (2015) wurden die Merkmale Umfang und Leistungspunkte herangezogen und ins Verhältnis gesetzt. Das als relativer Workload bezeichnete Verhältnis gibt an, wie hoch der Workload je Praktikumswoche ist. Im Ergebnis konnten drei Typen identifiziert werden:

Typ 1: Standorte, die Praktika einem vergleichsweise hohen Workload je Praktikumswoche, vorhalten. Die Praktikumsphasen dieser Standorte sind durch wenige, relativ kurze Praktika gekennzeichnet. Der Schwerpunkt der Praktika liegt, sowohl bezogen auf den Umfang als auch bezogen auf die Leistungspunkte der Praktika, im Masterstudium.

Typ 2: Standorte, die Praktika mit einem mittleren relativen Workload je Praktikumswoche vorhalten. Die Praktikumsphasen der Standorte dieses Typs sind durch vergleichsweise viele und kurze Praktika gekennzeichnet, deren Schwerpunkt in der Bachelorphase liegt.

Typ 3: Standorte, die Praktika mit einem relativ niedrigen Workload je Praktikumswoche vorhalten. Die Studierenden sind über einen längeren Zeit-

7 Gefragt wurde, ob die jeweiligen Einzelpraktika im Bachelor- und Masterstudium in ein praktikumsübergreifendes Konzept oder ein Schulpraxissemesterkonzept i.S. der unter 3.2. aufgegriffenen Definition eingebungen sind. Das Konstrukt praktikumsübergreifendes Konzept wurde bewusst nicht weiter eingeschränkt, da erfragt werden sollte, ob die Standorte ein - wie auch immer gestaltetes - übergreifendes Konzept einsetzen. Zur Differenzierung der Konzepte sind weitere Untersuchungen auf Basis studiengangsrelevanter Dokumente notwendig. 
raum an der Schule, bekommen aber im Durchschnitt nicht mehr Leistungspunkte als Studierende, die kurze bis mittlere Praktikumsphasen durchlaufen.

Im Ergebnis zeigt sich, dass diese Typenbildung sich als wenig aussagekräftig erweist, auch die Güte ist aufgrund des geringen Stichprobenumfangs als mittelmäßig einzuschätzen. Um zu besseren Ergebnissen zu kommen, wäre es denkbar, die Einzelpraktika als Einzelfälle zu betrachten, statt - wie hier erfolgt - die Standorte als Einzelfälle für die Typenbildung zugrunde zu legen.

\section{Schlussfolgerungen}

Ein Randphänomen ist die schulpraktische Ausbildung im universitären Lehramtsstudium größtenteils nicht mehr, die curriculare Einbindung durch Seminare und praktikumsübergreifende Konzepte ist zwar heterogen, aber es zeigt sich auch, dass das Schulpraktikum seine randständige Stellung im Studium verlässt und an Bedeutung gewinnt. Dennoch zeigt die Untersuchung, dass auch für das Lehramt an berufsbildenden Schulen das Bild der schulpraktischen Ausbildungsmodelle als Flickenteppich bezeichnet werden kann. Insgesamt hat sich in den Reformen der Studienprogramme ein breites Spektrum an Schulpraxisphasen und -konzepten etabliert. Alle Standorte zeichnen sich durch eine Einzigartigkeit aus, die sich teilweise nur schwer über ein Fragebogendesign erheben und abbilden lassen. Zwar sind die Merkmale und Indikatoren durchaus zur Beschreibung und zur Kategorisierung der Praxismodelle geeignet, die jeweiligen Ausprägungsformen dennoch teilweise so vielfältig und standortspezifisch, dass eine eindeutige Zuordnung im Einzelfall mitunter schwerfiel. Darüber hinaus muss eingeschränkt werden, dass das vorgestellte Konzept zur Beschreibung und Kategorisierung der Schulpraxisphasenmodelle nicht dazu geeignet ist, die einzelnen Schulpraxisphasenkonzepte in der realen Komplexität darzustellen und zu vergleichen. Jedoch zeigte sich, dass schon die kleine Auswahl an Merkmalen und Indikatoren eine große Diversität der Konzepte nachweisen konnte. Dies verdeutlicht die besonderen Herausforderungen struktureller Untersuchungen schulischer Praxisphasen. Dennoch konnten grundlegende Strukturen erfasst und abgebildet und damit eine aktuelle Bestandsaufnahme bezogen auf ausgewählte Merkmale vorgelegt werden, auf deren Basis weitere und vertiefende Untersuchungen vorgenommen werden können. Denkbare wären vertiefende Analysen der Praktikumsmodelle auf Basis studiengangsrelevanter Dokumente.

Für die Berufs- und Wirtschaftspädagogik zeigen die Ergebnisse der Untersuchung, dass im Feld der Wirksamkeitsforschung der schulpraktischen Studien bezogen auf die berufliche Lehramtsausbildung und standortüber- 
greifende Forschungsansätze ein deutliches Defizit besteht. Dies wird auch durch die Vielfalt der existierenden Praxisphasenmodelle an den Standorten deutlich. Lägen mehr Befunde zur wirksamen Ausgestaltung der schulischen Praxisphasen vor, wären standortübergreifend Modelle zu erwarten, die auf Forschungsbefunden aufbauen und somit eine systematische Professionalisierung befördern und gleichzeitig die Mobilität der Studierenden verbessern könnten.

Im Beitrag wurden einige Ansätze für weitergehende Forschungsfragen angeschnitten, interessant ist es hier vor allem für die Qualitätsverbesserung der Praxisphasen in der beruflichen Lehramtsausbildung die Besonderheiten der beruflichen Bildung einzubeziehen. Forschungsmethodisch stellen derartige Forschungsfragen jedoch eine große Herausforderung dar. Längsschnittstudien und standortübergreifende Untersuchungen werden in der Wirksamkeitsforschung als fruchtbare Untersuchungsdesigns aufgefasst, die es erlauben, Wirkungsmodelle und -zusammenhänge zu identifizieren, zu beschreiben und folglich Lehrerausbildungsmodelle zu vergleichen (vgl. Gröschner, Schmitt 2010).

Zwar ist die Erforschung der Praxisphasen im Lehramtsstudium mittlerweile zu einem florierenden Forschungsfeld herangereift, die Zahl der Untersuchungen, die die Spezifika des Lehramtes an berufsbildenden Schulen explizit berücksichtigen, ist jedoch im Vergleich zu anderen Lehramtstypen sehr gering.

\section{Literatur}

Bach, A., Besa, K., Arnold, K.-H. (2014). Bedingungen von Lernprozessen in Schulpraktika: Ergebnisse aus dem Projekt ESIS (Entwicklung Studierender in Schulpraktika). In: K.-H. Arnold, A. Gröschner, T. Hascher (Hrsg.), Schulpraktika in der Lehrerbildung. Theoretische Grundlagen, Konzeptionen, Prozesse und Effekte, (S. 165-182). Münster, New York: Waxmann.

Bargel, T. (2012). Bedeutung von Praxisbezügen im Studium. In: Schubarth, W., Speck, K., Seidel, A., Gottmann, C., Kamm, C., Krohn, M. (Hrsg.), Studium nach Bologna: Praxisbezüge stärken?! Praktika als Brücke zwischen Hochschule und Arbeitsmarkt. (S. 37-46). Wiesbaden: Springer.

BaSS (Bundesarbeitsgemeinschaft Schulpraktische Studien) (2011). Stellungnahme der Bundesarbeitsgemeinschaft Schulpraktische Studien zu Langzeitpraktika. Online: http://www.diz.uni-oldenburg.de/BaSS/download/Stellungnahmen/ Langzeitpraktika_Marburg_2011.pdf (05.07.2016).

Becker, M., Spöttl, G. (2013). Lehrerbildung in beruflichen Fachrichtungen - Gemeinsamkeiten, Besonderheiten, Differenzierungen, Standards. Berufs- und Wirtschaftspädagogik online, Spezial 6, 1-10. Online: http://www.bwpat.de/hat2013/ ws19/becker_spoettl_ws19-hat2013.pdf (08.04.2017). 
Bennack, J., Jürgens, E. (2002). Schulpraktika in Lehramtsstudiengängen. In: H.-U. Otto, K. Bock (Hrsg.), Erziehungswissenschaft: Lehre und Studium (S. 143-160). Opladen: Leske + Budrich.

Besa, K.-S., Büdcher, M. (2014). Empirical evidence on field experiences in teacher education: a review oft he research base. In: K.-H. Arnold, A. Gröschner, T. Hascher (Hrsg.), Schulpraktika in der Lehrerbildung. Theoretische Grundlgen, Konzeptionen, Prozesse un Effekte (S. 129-145). Münster, New York: Waxmann.

Gröschner, A., Müller, K., Bauer, J., Seidel, T., Prenzel, M., Kasper, T. \& Möller, J. (2015). Praxisphasen in der Lehrerausbildung - Eine Strukturanalyse am Beispiel des gymnasialen Lehramtsstudiums in Deutschland. Zeitschrift für Erziehungswissenschaft, 38, 639-665.

Gröschner, A., Schmitt, C., Seidel, T. (2013). Veränderung subjektiver Kompetenzeinschätzungen von Lehramtsstudierenden im Praxissemester. Zeitschrift für Pädagogische Psychologie, 27(1-2), 77-86.

Gröschner, A., Schmitt, C. (2010). Wirkt, was wir bewegen? - Ansätze zur untersuchung der Qualität universitärer Praxisphasen im Kontext der Reform der Lehrerbildung. Erziehungswissenschaft. Mitteilungen der Deutschen Gesellschaft für Erziehungswissenschaft (DGfE), 21(40), 89-97.

Hascher, T. (2012a). Lernfeld Praktikum - Evidenzbasierte Entwicklungen in der Lehrer/-innenbildung. Zeitschrift für Bildungsforschung, 2, 109-129.

Hascher, T. (2012b). Forschung zur Bedeutung von Schul- und Unterrichtspraktika in der Lehrerinnen- und Lehrerbildung. Beiträge zur Lehrerbildung, 30(1), 87-98.

Hascher, T. (2011). Vom „Mythos Praktikum“ und der Gefahr verpasster Lerngelegenheiten. Journal für Lehrerinnen- und Lehrerbildung, 3, 8-16.

Hascher, T. (2006). Veränderungen im Praktikum - Veränderungen durch das Praktikum. Eine empirische Untersuchung zur Wirkung schulpraktischer Studien in der Lehrerbildung. Zeitschrift für Pädagogik, 51. Beiheft, 130-148.

Hedtke, R. (2000). Das unstillbare Verlangen nach Praxisbezug - Zum TheoriePraxis-Problem der Lehrerbildung als Exempel Schulpraktischer Studien. In: H. J. Schlösser (Hrsg.), Berufsorientierung und Arbeitsmarkt (S. 67-91). Bergisch Gladbach.

Helsper, W., Breidenstein, G., Kotters-König, C. (2002). Einleitung. In: G. Breidenstein, W. Helsper, C. Kotters-König (Hrsg), Die Lehrerbildung der Zukunft-eine Streitschrift. Opladen: Leske + Budrich.

Holtz, P., Kleinespel, K., Ahrens, F., Luetgert, W. (2013). „Echte Lehrer“ vs. „Schnuli-Bulli-Theorie“: Das Spannungsfeld zwischen Theorie und Praxis im Lehramtsstudium im Spiegel des Jenaer Praxissemesters. Online: http://www.lbf.unijena.de/zldmedia/Downloads/Forschung/ZLB_Beitrag_Theorie_Praxis_Diskussion_Praxissemester.pdf (08.04.2017).

HRK (Höhschulrektorenkonferenz) (1998). Empfehlungen zur Lehrerbildung. 186. Plenum der HRK vom 2. November 1998.

KMK (Kultusministerkonferenz) (2016). Rahmenvereinbarung über die Ausbildung und Prüfung für ein Lehramt der Sekundarstufe II (berufliche Fächer) oder für die beruflichen Schulen (Lehramtstyp 5). Beschluss der Kultusministerkonferenz vom 12.05.1995, i.d.F. vom 06.10.2016.

KMK (Kultusministerkonferenz) (2013). Empfehlungen zur Eignungsabklärung in der ersten Phase der Lehrerausbildung. Beschluss der Kultusministerkonferenz vom 07.03.2013. 
KMK (Kultusministerkonferenz) (2005). Eckpunkte für die egenseitige Anerkennung von Bachelor- und Masterabschlüssen in Studiengängen, mit denen die Bildungsvoraussetzungen für ein Lehramt vermittelt werden. Beschluss der Kultusministerkonferenz vom 02.06.2005.

Lange, S. (i. E.): Strukturen schulischer Praxisphasen im beruflichen Lehramtsstudium in Deutschland. Beitrag im Tagungsband zum 3./4. Chinesisch-Deutschen Berufsbildungsworkshop.

Offenberg, E., Walke, J. (2013). Die Reform der Praxisphasen in der Ersten Phase der Lehrerbildung. Eine qualitative Dokumentenanalyse. Bonn.

Perle, H.-J. (Hrsg.) (1999). Schulpraktische Studien und Praxissemester. Ergebnis gemeinsamer Beratungen auf der 19. Bundestagung der Leiter der Praktikumsbüros am 20. Mai 1999 an der Technischen Universität Dresden. Göttingen.

Rexing, V., Klitze, M. (2015). Forschendes Lernen im Praxissemester - Umsetzung in der Fakultät für Bauingenieurwesen der RWTH Aachen. BAG-Report, 2, 41-49.

Schubarth, W., Speck, K., Seidel, A., Gottmann, C., Kamm, C., Krohn, M. (2012a). Praxisbezüge im Studium - Ergebnisse des ProPrax-Projektes zu Konzepten und Effekten von Praxisphasen unterschiedlicher Fachkulturen. In: Schubarth, W., Speck, K., Seidel, A., Gottmann, C., Kamm, C., Krohn, M. (Hrsg.), Studium nach Bologna: Praxisbezüge stärken?! Praktika als Brücke zwischen Hochschule und Arbeitsmarkt (S. 47-100).Wiesbaden: Springer.

Schubarth, W., Speck, K., Seidel, A., Gottmann, C., Kamm, C., Krohn, M. (2012b). Das Praxissemester im Lehramt - ein Erfolgsmodell? Zur Wirksamkeit des Praxissemesters im Land Brandenburg. In: Schubarth, W., Speck, K., Seidel, A., Gottmann, C., Kamm, C., Krohn, M. (Hrsg.), Studium nach Bologna: Praxisbezüge stärken?! Praktika als Brücke zwischen Hochschule und Arbeitsmarkt (S. 137-169). Wiesbaden: Springer.

Terhart, E. (2013). Vorwort. In E. Offenburg, J. Walke (Hrsg.), Die Reform der Praxisphasen in der Ersten Phase der Lehrerbildung. Eine qualitative Dokumentenanalyse. Bonn.

Weyland, U. (2014). Schulische Praxisphasen im Studium: Professionalisierende oder deprofessionalisierende Wirkung? Berufs- und Wirtschaftspädagogik online, Profil 3, 1-24. Online: www.bwpat.de/profil3/weyland_profil3.pdf (01.11.2016).

Weyland, U. (2012). Expertise zu den Praxisphasen in der Lehrerbildung in den Bundesländern. Hamburg: Landesinstitut für Lehrerbildung und Schulentwicklung.

Weyland, U. (2010). Zur Intentionaität Schulpraktischer Studien im Kontext universitärer Lehrerausbilung. Paderborn: Eusl.

Weyland, U., Wittman, E. (2010). Praxissemester im Rahmen der Lehrerbildung 1. Phase an hessischen Hochschulen. Expertise. Berlin: DIPF. 



\title{
Handlungsempfehlungen aus dem Einsatz und der Evaluation eines Tagungsportfolios im Rahmen der Lehrerbildung
}

\author{
Heike Jahncke und Karina Kiepe
}

\section{Einleitung}

Im Rahmen eines Bundeskongresses des Bundesarbeitskreises für Seminarund Fachleiter/-innen (BAK) 2015 wurde in Anlehnung an das Motto „We do not learn from experience... we learn from reflecting on experience" (Dewey 1933) und den Ansatz von Schön (1983), der das Idealbild einer ihr Handeln reflektierenden und erforschenden Lehrperson im Sinne des reflective practitioner propagiert, für alle Teilnehmer/-innen ein Reflexionsportfolio zur Verfügung gestellt und im Nachgang evaluiert. Das Reflexionsportfolio wurde explizit für den Einsatz bei einer Tagung entwickelt und wird deshalb nachfolgend als Tagungsportfolio bezeichnet. Recherchen ergaben, dass ein Tagungsportfolio bisher weder im deutschsprachigen noch im fremdsprachigen Raum eingesetzt wurde. Bei der Konzeption des Tagungsportfolios konnte allerdings auf vorhandene Portfolios mit ähnlichen Zielsetzungen aufgebaut werden (vgl. u. a. Jahncke 2015, 14 ff.). Die Entwicklung der Reflexionsanlässe des Tagungsportfolios erfolgte dabei unter der Maßgabe, dass diese an die vielfältigen Angebote der Tagung anknüpfen sollten (vgl. Abschnitt 2). Der Umstand, dass Portfolios bereits vielfach an Universitäten und anderen Hochschulen in der Lehrerbildung verankert sind oder ihr Einsatz zumindest in Erwägung gezogen wird, stützt das Vorhaben, Portfolios im Rahmen von Tagungen einzusetzen (vgl. z. B. van Dellen 2013, 53).

$\mathrm{Zu}$ den derzeit bekannten Einsätzen des Portfoliokonzeptes liegen bereits erste Forschungsergebnisse aus unterschiedlichen Kontexten vor, bei denen es sich überwiegend um Erfahrungsberichte handelt. Allerdings brachte eine bundesweite Untersuchung des Fachgebietes Berufs- und Wirtschaftspädagogik der Universität Oldenburg für die Konzeption des Tagungsportfolios mittels einer Onlinebefragung ausschlaggebende Ergebnisse hervor (vgl. Jahncke, Slopinski \& Hüsing 2015, 39 ff.). An der Befragung nahmen 312 Lehrkräfte im Vorbereitungsdienst aus elf Bundesländern teil, von denen ca. die Hälfte 30 Jahre oder jünger war. Die Befragten erwarben die Lehrbefähigung in einer der folgenden Fachrichtungen: (1) Wirtschaftswissenschaften, (2) haushalts- und personenbezogene Dienstleistungen oder (3) gewerblichtechnische Fachrichtung. Die Befragung zeigte, dass der Einsatz des Portfo- 
lios im Vorbereitungsdienst zwar angenommen wird, aber noch nicht vollständig ausgereift ist. Dabei fiel besonders auf, dass für eine Vielzahl von Referendar(inn)en die Arbeit mit dem Portfolio durch fehlende Wertschätzung seitens ihrer Fachleiter/-innen geprägt ist und diese zudem oftmals den Eindruck vermitteln, dass die Portfolioarbeit nicht von ihnen erwünscht, sondern vielmehr allein aufgrund von Vorgaben umgesetzt wird. Optimiert werden kann die Arbeit mit dem Portfolio der Untersuchung zufolge durch eine stärkere Integration in den Seminaralltag, um sie sichtbar und dauerhaft als Selbstverständlichkeit im Vorbereitungsdienst zu verankern (vgl. Jahncke, Slopinski \& Hüsing 2015, 41).

Gemäß dem Credo: Jede gute Umsetzung fängt bei den Verantwortlichen an, waren diese Ergebnisse Anlass genug, Fachleiter/-innen bewusst selbst mit dem Portfolio arbeiten zu lassen. Denn nur durch das Sammeln eigener Erfahrungen können sie das nötige Wissen und die Routinen aufbauen, um auch ihre Referendare/Referendarinnen unterstützend an die Arbeit mit dem Portfolio heranzuführen und die passende Rahmung für den Einsatz eines Portfolios zu schaffen. Dies folgt der Maßgabe von Neuweg (2017, 98 f.), wonach der Lernfortschritt nicht auf Theorieebene beginnen kann, sondern ein Veränderungsprozess an eigenen Erlebnissen ansetzt. So kann die notwendige Akzeptanz und Wertschätzung für die Portfolioarbeit nur im eigenen Handeln entwickelt werden. Ein solches Vorgehen wird dann auch einem konstruktivistischen Verständnis von Lernen gerecht. So wird den Fachleiter(inne)n durch die Arbeit mit dem Portfolio im Rahmen der Tagung die Möglichkeit gegeben, sich auf Basis eigener Erfahrungen und Wahrnehmungen mit dem Portfoliokonzept auseinanderzusetzen, ihr Wissen darüber zu erweitern und durch Handlung zu überprüfen (vgl. Rebmann \& Schlömer 2010,9).

Der nachfolgende Beitrag widmet sich neben der Darstellung der Entwicklung, des Einsatzes sowie der Evaluation des Tagungsportfolios auch der Ableitung von Handlungsempfehlungen für den zukünftigen Portfolioeinsatz in der Lehrerbildung. Darüber hinaus werden neue Forschungsdesiderata mit Blick auf die Portfolioarbeit in der Lehrerbildung aufgezeigt.

\section{BAK-Bundeskongress als Einsatzfeld des Tagungsportfolios}

Beim BAK-Bundeskongress 2015 handelte es sich um die 49. Tagung des Bundeskreises der Seminar- und Fachleiter/-innen (vgl. BAK 2015) mit insgesamt 296 Teilnehmer(inne)n. Der inhaltliche Schwerpunkt der Tagung lag auf dem Themenfeld Reflektieren und Beraten. Dies liegt darin begründet, dass qualifizierte Methoden und Kriterien von Beratung und Reflexion im- 
mer mehr zur Voraussetzung wirksamen pädagogischen Handelns und damit Kern berufsbezogener Professionalität werden (vgl. Koch-Priewe 2013, 42 f.). Im Rahmen der viertägigen Tagung wurden vielfältige Möglichkeiten von Reflexions- und Beratungsverfahren bezogen auf unterschiedliche Anspruchsgruppen bearbeitet und gemeinsam mit renomierten Referent(inn)en diskutiert. Konkrete Themen auf der Agenda waren zum Beispiel: Systematisierung und Reflexion, Feedback im Unterricht oder Das Konstrukt ,, Reflexion " - Theoretische Grundlagen und empirische Ergebnisse. Mit Blick auf diese Themen wird deutlich, warum thematisch der Einsatz eines Tagungsportfolios mit gezielten Reflexionsanlässen zu den Veranstaltungen der Tagung nahelag.

Nachfolgend wird aufgezeigt, welche Punkte im Allgemeinen bei der Konzeption und Einführung eines Portfolios zu beachten sind und wie diese im Speziellen für das eingesetzte Tagungsportfolio umgesetzt wurden.

\section{Theoretischer Bezugsrahmen und Konzeption des Tagungsportfolios}

Trotz der starken Präsenz des Portfolios im pädagogischen Kontext existiert keine eindeutige Definition, und auch die Inhalte der Portfolioarbeit lassen sich nicht klar abgrenzen (vgl. z. B. Koch-Priewe 2013, 42; Schwenk 2012, 66). Exemplarisch lässt sich hier Häcker $(2007,127)$ anführen, der im Zuge eines Definitionsversuchs auf Paulson et al. $(1991,60)$ verweist: „Ein Portfolio ist eine zielgerichtete Sammlung von Arbeiten, welche die individuellen Bemühungen, Fortschritte und Leistungen der/des Lernenden auf einem oder mehreren Gebieten zeigt. Die Sammlung muss die Beteiligung der/des Lernenden an der Auswahl der Inhalte, der Kriterien für die Auswahl, der Festlegung der Beurteilungskriterien sowie Hinweise auf die Selbstreflexion der/des Lernenden einschließen." Getragen durch den Umstand, dass sich das Portfolio aus der Praxis heraus entwickelt hat, lassen sich in der Literatur weiterhin eine Vielzahl von Klassifizierungsmöglichkeiten von Portfolioarten vorfinden. Diese Vielzahl verdeutlicht ebenfalls, dass es „das“ eine Portfolio nicht gibt. Vielmehr werden die Art des Portfolios sowie seine Bezeichnung nach dem jeweiligen Verwendungszweck und Einsatzgebiet definiert (vgl. z. B. Winter 2013, 16 ff.). In der Praxis treten dann zumeist Mischformen auf, da das Portfolio häufig unterschiedliche Funktionen erfüllen soll (vgl. Gläser-Zikuda \& Hascher 2007, 13). Losgelöst davon, in welcher Funktion ein Portfolio betrachtet wird und welche Definition dem Portfolio zugrunde liegt, ist die Entscheidung für oder gegen dessen Einsatz immer eine didaktische Überlegung. Das bedeutet, dass darüber Auskunft gegeben werden muss, welche übergeordneten Bildungs- und Lernziele durch den Einsatz 
eines Portfolios realisiert werden sollen (vgl. Häcker \& Seemann 2013, 77). Infolgedessen muss der Einsatz von Portfolios umfassend geplant werden. Mit dem Ziel, die Umsetzung der Portfolioarbeit in praxi zu unterstützen, hat das Internationale Netzwerk Portfolioarbeit auf Grundlage von Erfahrungen mit dem Portfoliokonzept in Deutschland sowie in Österreich geeignete Qualitätskriterien formuliert, die bei der Planung, Analyse und Evaluation Orientierungshilfe leisten (vgl. z. B. Winter 2007, 373).

Wie Abbildung 1 zeigt, sind die Orientierungspunkte für die Planung, Durchführung sowie Evaluation des Tagungsportfolios in drei Kategorien zusammengefasst. Winter $(2007,374)$ weist dabei ausdrücklich darauf hin, dass die Unterteilung der einzelnen Merkmale in einzelne Kategorien nicht als Disjunktion oder strenge zeitliche Abfolge zu verstehen ist. Vielmehr bestehen zwischen den einzelnen Merkmalen enge Beziehungen, wie sich auch aus den Pfeilen in der Abbildung 1 ableiten lässt. Nachfolgend werden diejenigen dieser Merkmale aufgegriffen, die bei der Entwicklung des Tagungsportfolios sowie seiner Rahmung besondere Berücksichtigung fanden.

Abb. 1: Orientierungspunkte für die Portfolioarbeit

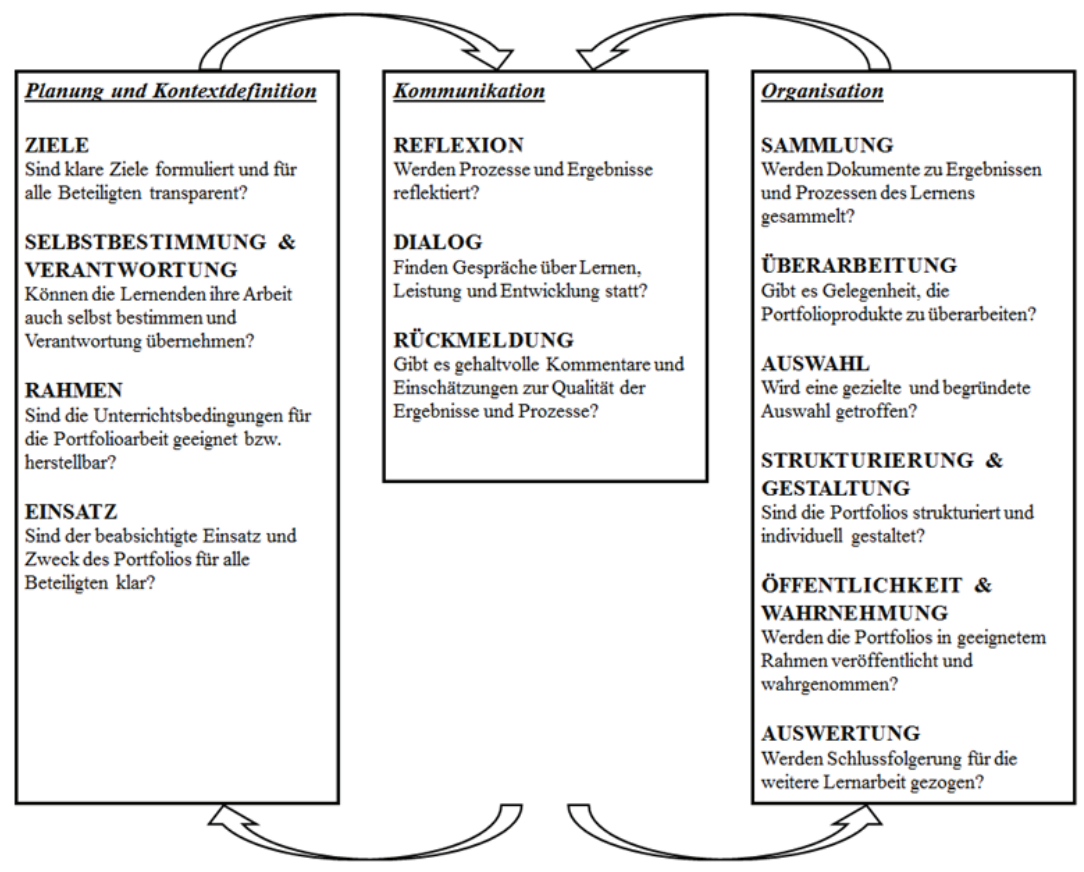

Quelle: Winter (2007, 374) 
Für die Merkmalskategorie Planung und Kontextdefinition ist anzuführen, dass im Rahmen der Planung sowie der Einführung des Tagungsportfolios besonders darauf geachtet wurde, dass die Rahmenbedingungen für den Einsatz eines solchen Portfolios gegeben waren, indem z. B. Zeitfenster für die Reflexionsanlässe geschaffen wurden sowie eine Portfoliomappe mit den einzelnen Reflexionsanlässen und Schriftfeldern erstellt und ausgegeben wurde. Zudem wurden die Teilnehmer/-innen zu Beginn der Tagung eingehend über die Intentionen des Einsatzes des Tagungsportfolios informiert. Rückfragen wurden gemeinsam mit den Teilnehmer/-innen besprochen (vgl. zur Notwendigkeit der Einführung auch u. a. Bräuer 2014, 4 f.; Leonhard 2013, 183).

Bezugnehmend auf die Merkmalskategorie Kommunikation, ist das Merkmal der Reflexion hervorzuheben, welches das Herzstück der Portfolioarbeit darstellt (vgl. z. B. Tettenborn 2014, 42; Winter 2007, 376). Das professionelle Reflektieren ist ein bewusstes Nachdenken vor, während oder auch nach einer bestimmten Situation und/ oder Handlung (vgl. Henning \& Kricke 2016, 37). Es kann schriftlich oder gedanklich stattfinden und sich mit stark variierenden Inhalten befassen. Die Fähigkeit, zu reflektieren, ist dabei eine wichtige Komponente der Problemlösungskompetenz für das berufliche Handeln von ,ganzheitlich denkende[n] und handelnde[n] Persönlichkeiten [...], die über ein Wissen und Können verfügen müssen, das sie zur Bewältigung laufender neuer und unerwarteter Situationen befähigt" (Dubs 2006, 191). Reflexion erfordert dabei entsprechende Spielräume, d. h. eine eigene Rahmung, welche eine handlungsentlastende, distanzierte Auseinandersetzung ermöglicht (vgl. Häcker 2012, 271). Im Wissen darum wurden im Rahmen der Tagung nicht nur Portfoliozeiten zum Reflektieren eingeplant, sondern auch passend zu den Angeboten der Tagung Reflexionsanlässe entwickelt, um die Reflexion über das Gehörte, Gesehene oder auch selbst Durchgeführte zu stimulieren. Exemplarisch für diesen auf den individuellen Erfahrungen basierenden Ansatz steht der folgende Reflexionsanlass: Welche Inhalte/Themen des Vortrages waren besonders bedeutsam für mich? Was kann ich von den neuen Inhalten in mein berufliches Handeln integrieren? Wie könnte das funktionieren? Bei der Konzeption der Reflexionsanlässe sahen sich die Entwicklerinnen mit der Problematik konfrontiert, dass sich in der Fachliteratur nur vereinzelt Ausführungen zur konkreten Ausgestaltung von Reflexionsanlässen bzw. Handlungsanleitungen für das Reflektieren im Portfolio finden lassen (vgl. z. B. Häcker 2005, 6).

Bezugnehmend auf die Merkmalskategorie Organisation, ist festzuhalten, dass diese Merkmale subsumiert, die sich insbesondere auf die Organisation des Portfoliokonzepts beziehen. Für die Ausgestaltung des Tagungsportfolios sind aufgrund seiner speziellen Form nicht alle Charakteristika erfüllbar. Hervorzuheben ist aber das Merkmal der Auswertung, bei dem von der reflektierenden Person Schlussfolgerungen für nachfolgende Lernprozesse 
gezogen werden. Übertragen auf das Tagungsportfolio wurde diesem Merkmal in doppelter Hinsicht Genüge geleistet: zum einen, indem die Teilnehmer/-innen am Ende eines jeden Tages dazu aufgefordert wurden, Bilanz über die Erkenntnis des Tages zu ziehen und dabei ein zentrales Element herauszufiltern, das sie für ihr berufliches Handeln aufnehmen wollen. Dieses Vorgehen zielt sehr explizit auf die Selbsterfahrung der Teilnehmer/-innen $\mathrm{ab}$, indem sie durch die eigene Arbeit mit dem Tagungsportfolio in eine Situation gebracht wurden, wie sie sonst Referendare/Referendarinnen im Rahmen ihrer Studienseminare erleben. Damit wurde eine Parallele zur Tätigkeit der Fachleiter/-innen außerhalb der Tagung hergestellt.

Zum anderen wurde die Merkmalskategorie der Organisation adressiert, indem abschließend in einem letzten, von den Vorträgen, Workshops und dem Unterrichtsbesuch losgelösten, Reflexionsanlass von den Teilnehmer(inne)n bewertet wurde, welche Ziele und Erwartungen sie mit der Teilnahme an den Kongress verbanden und ob diese erreicht wurden. In diesem Zusammenhang wurden sie zudem dazu aufgefordert, für sich festzuhalten, welche Aspekte sie für ihr zukünftiges berufliches Handeln aufnehmen möchten. Der Reflexionsanlass erreichte dies, indem er dazu aufforderte, Bilanz zu ziehen und die Tagung entlang der folgenden Impulse rückblickend $\mathrm{zu}$ evaluieren: Was waren meine Ziele und inwieweit habe ich meine Ziele erreicht? Was waren meine Erwartungen und haben sich diese erfüllt? Folgende Themen und Inhalte standen völlig im Gegensatz zu meinen Erwartungen... . Für mein zukünftiges berufliches Handeln möchte ich folgende Aspekte mehr in den Fokus nehmen... . Das ist mein erster Schritt... . Mit folgenden Personen möchte ich in Kontakt bleiben bzw. in Zusammenarbeit gehen... .

Die einzelnen Reflexionsanlässe trugen durch ihre Ausgestaltung zudem dazu bei, die Teilnehmer/-innen auch im Nachgang des BAK-Bundeskongresses dazu anzuhalten, die Inhalte des Kongresses immer wieder unter Bezugnahme auf ihr eigenes Handeln zu reflektieren und in diesem Zusammenhang Ziele sowie Strategien für deren Umsetzung zu entwickeln. Darüber hinaus stellte das Tagungsportfolio erste Gestaltungshinweise für die Ausgestaltung eines möglichen Portfoliokonzeptes im Vorbereitungsdienst zur Verfügung und ermöglichte den Seminar- und Fachleiter(inne)n, ihre eigenen Erfahrungen mit der Portfolioarbeit an ihre Referendare/ Referendarinnen weiterzugeben. 


\section{Zusammenfassende Ergebnisse der Evaluation des Tagungsportfolios}

Um die Effekte aus dem Einsatz des Tagungsportfolios bewerten zu können, wurde es im Nachgang mit Blick auf die folgenden zwei Fragestellungen evaluiert:

1. Wie hat sich die Einstellung der Fachleiter/-innen zum Portfolio durch die Arbeit mit dem Tagungsportfolio verändert?

2. Welchen Beitrag leistete der Einsatz des BAK-Tagungsportfolios für die zukünftige Ausgestaltung der Portfolioarbeit in der Arbeit der Fachleiter/-innen?

Zur Untersuchung dieser Fragestellungen wurde ein zweistufiges Evaluationsverfahren verwendet. In einem ersten Schritt erfolgte mittels einer Onlinebefragung unmittelbar nach der Tagung eine erste Einschätzung zur Nutzung und ersten Bewertung des Tagungsportfolios durch die Teilnehmer/innen. Erster Block der Onlinebefragung war zunächst eine Abfrage der biografischen Daten der Befragten wie beispielsweise Alter, Berufserfahrung und unterrichtete Schulformen. Die darauf folgenden Items zielten zunächst auf eine Gesamtevaluation der Tagung ab, indem die Teilnehmer/-innen z. B. nach ihrer Zufriedenheit mit der Ausgestaltung, der Organisation sowie den Inhalten der Tagung gefragt wurden. Daran anschließend wurden konkrete Items zum Tagungsportfolio platziert. Hier wurden neben den Portfolioerfahrungen der Teilnehmer/-innen auch ihre Einstellungen zur Portfolioarbeit vor der Tagung sowie ihre Einstellung zur Portfolioarbeit nach der Tagung abgefragt, um gezielt einen möglichen Wandel bewerten zu können. Darüber hinaus waren auch Items enthalten, die sich auf die Ausgestaltung des Tagungsportfolios bezogen.

An der Onlinebefragung nahmen 160 Personen teil, dies entspricht einer Rücklaufquote von $54 \%$. Im Kern brachte die Onlinebefragung hervor, dass das Tagungsportfolio von insgesamt $66 \%$ der Befragten genutzt wurde. Eine Befürwortungsquote von ca. 85 \% für den generellen Einsatz eines Tagungsportfolios ließ den ersten Schluss zu, dass die Idee, Tagungsportfolios auch zukünftig einzusetzen, weiterhin verfolgt werden sollte und die konkret gewählte Gestaltung eine gute Basis bildet.

Im zweiten Schritt wurden anschließend an die Onlinebefragung problemzentrierte Interviews durchgeführt. Die Auswahl der Interviewpartner/innen wurde dabei mit einem qualitativen Stichprobenplan (vgl. z. B. Döring \& Bortz 2016, 303) aus einem Pool von 67 Personen vorgenommen, die sich im Rahmen der Onlinebefragung für ein Interview bereit erklärt hatten. Insgesamt wurden neun Personen interviewt. Diese Stichprobe setzte sich dabei wie folgt zusammen: (1) Drei Personen, die bei der Onlinebefragung anga- 
ben, vor der Nutzung des Tagungsportfolios noch keine Erfahrungen mit der Portfolioarbeit gemacht zu haben. (2) Drei Personen, die bei der Onlinebefragung angaben, vor der Nutzung des Tagungsportfolios bereits Erfahrungen mit der Portfolioarbeit gemacht zu haben und diese als positiv bewerteten. (3) Drei Personen, die bei der Onlinebefragung angaben, vor der Nutzung des Tagungsportfolios bereits Erfahrungen mit der Portfolioarbeit gemacht zu haben und diese als negativ bewerteten.

Mit Blick auf die erste Frage, welche Auswirkungen das Tagungsportfolio auf die Einstellung der Fachleiter/-innen gegenüber der Portfolioarbeit hat, ist festzuhalten, dass das Tagungsportfolio bei zwei Personen eine positive Veränderung der Einstellung bewirkte. Für den Rest der Befragten ergab sich keine identifizierbare Veränderung. An dieser Stelle ist jedoch anzuführen, dass die Interviews erkennen ließen, dass alle befragten Fachleiter/-innen bereits vor der Tagung eine durchweg positive Einstellung zum Konzept des Portfolios hatten, selbst, wenn die bisherigen Erfahrungen negativ waren. Die im Zuge der Onlinebefragung erhobene negative Perspektive einzelner Personen gegenüber der Portfolioarbeit bezog sich ausschließlich auf die praktische Umsetzung der Portfolioarbeit im Studienseminar im Einzelfall und nicht auf das Konzept der Portfolioarbeit im Allgemeinen. So monierten die Fachleiter/-innen u. a. die zeitlichen Rahmenbedingungen für den Einsatz von Portfolios im Studienseminar sowie die defizitären Materialien (z. B. fehlende Anleitung für den Portfolioeinsatz, fehlende Hinweise zur Ausformulierung von Reflexionsanlässen usw.), die für die Portfolioarbeit im Studienseminar vorhanden sind. Selbst die Befragten, die angaben, keine Vorerfahrungen mit Portfolioarbeit zu haben, ließen bezüglich des Portfoliokonzeptes eine positive Einstellung erkennen. Diese Erkenntnis ist im Besonderen vor dem Hintergrund der eingangs beschriebenen Forschungsergebnisse erstaunlich, in denen Referendare/Referendarinnen eine fehlende Wertschätzung seitens der Fachleiter/-innen dem Portfoliokonzept gegenüber konstatierten (vgl. Abschnitt 1). Mit Blick auf die Ergebnisse der Interviews, ist diese Wahrnehmung der Referendare/Referendarinnen daher differenziert zu betrachten. Die interviewten Fachleiter/-innen haben dem Konzept des Portfolios grundsätzlich eine hohe Wertschätzung zugeschrieben. $\mathrm{Zu}$ vermuten ist daher, dass die von den Referendar(inn)en wahrgenommene fehlende Wertschätzung der Portfolioarbeit aus der Ablehnung der Fachleiter/-innen den Rahmenbedingungen gegenüber resultiert, in denen Portfolioarbeit im Studienseminar stattfindet.

Im Wissen darum gilt es, im Rahmen sich anschließender Untersuchungen mittels einer größeren Stichprobe zunächst zu untersuchen, ob die hier gewonnene Erkenntnis bestätigt werden kann. Ferner sind in diesem Zusammenhang auch die Rahmenbedingungen des Einsatzes von Portfolios im Studienseminar näher zu beleuchten. 
Mit Blick auf die zweite Fragestellung, welchen Beitrag der Einsatz des BAK-Tagungsportfolios für die zukünftige Ausgestaltung der Portfolioarbeit in der Arbeit der Fachleiter/-innen leistet, zeigt sich, dass das Tagungsportfolio keine unmittelbaren Auswirkungen auf die Ausgestaltung der Portfolioarbeit im Studienseminar hat. Vielmehr konnte die bereits eingangs skizzierte Wahrnehmung der Referendare/Referendarinnen, dass die Fachleiter/-innen den Eindruck vermitteln, Portfolioarbeit nur aufgrund von Vorgaben umzusetzen, durch die Analyse der Interviews bestätigt werden. Die drei Personen, die angaben, das Portfolio bereits zu nutzen, tun dies aufgrund rechtlicher Vorgaben. Ferner gab keine dieser drei Personen an, durch das Tagungsportfolio etwas in der eigenen Arbeit mit dem Portfolio ändern zu wollen. Trotz der hohen Wertschätzung dem Portfoliokonzept gegenüber und der positiven Bewertung des Tagungsportfolios, hat keine der übrigen sechs Personen im Anschluss an die Tagung ein Portfolio im Studienseminar eingeführt. Unter Rückbezug auf die Ergebnisse der Onlinebefragung, ist anzuführen, dass dort $82 \%$ der Befragten angaben, dass sie sich vorstellen können, das Portfolio zukünftig nutzen zu wollen. Diese Tendenz konnte durch die Interviews nicht bestätigt werden. Gleichwohl lassen sich vereinzelt indirekte Auswirkungen des Tagungsportfolios in Form von Diskussionen unter Fachleiter(inne)n über die Portfolioarbeit identifizieren.

Auf Basis der dargestellten Ergebnisse, der gewonnenen Erfahrungen im Rahmen der Konzeption eines Portfolios sowie der damit einhergehenden Literaturrecherche, wird nachfolgend der Schwerpunkt darauf gelegt, erste Handlungsempfehlungen für die Portfolioarbeit in der Lehrerbildung auszusprechen sowie Forschungsdesiderata auf dem Gebiet der Portfolioarbeit aufzuzeigen.

\section{Ausblick}

Zunächst ist festzuhalten, dass in wissenschaftlichen Diskursen viele Argumente ausfindig zu machen sind, die für den Portfolioansatz in unterschiedlichen Kontexten sprechen. Besonders hervorgehoben wird dabei die Beförderung der Reflexionsfähigkeit durch den Einsatz von Portfolioarbeit (vgl. z. B. Häcker 2011, 109). Wohl nicht zuletzt aus diesem Grund hält die Arbeit mit dem Portfolio seit Beginn des 21. Jahrhunderts Einzug in die ersten beiden Phasen sowie gelegentlich auch die dritte Phase der Lehrerbildung (vgl. z. B Hertle \& Sloane 2007, 11; Kraler 2007, 441; Tettenborn 2014, 43 f.). Die praktische Umsetzung des Portfoliokonzeptes hingegen trifft häufig auf Widerstände, da viele Lehrende die Portfolioarbeit nur als Maßgabe höherer Instanzen wahrnehmen und darum häufig eine ablehnende Haltung dazu aufbauen. Dieser Ablehnung versuchte das Tagungsportfolio zu begegnen, 
indem durch die eigene Arbeit mit dem Tagungsportfolio die Fachleiter/innen die Vorzüge des Portfoliokonzeptes selbst erfahren konnten. Diese Erfahrungen sollten sie dazu motivieren, die Portfolioarbeit künftig in ihre Arbeit zu integrieren, denn besonders die Selbsterfahrung der Teilnehmer/innen im Rahmen der Tagung stellt eine Möglichkeit dar, den Einsatz von Portfolios im Rahmen der eigenen Lehrveranstaltungen zu optimieren.

Ferner ist zu konstatieren, dass, obwohl die Reflexion als „Herzstück“ der Portfolioarbeit anerkannt ist, bisher nur unzureichend definiert wurde, was eigentlich unter dem Konstrukt Reflexion zu verstehen ist. In Folge einer unzureichenden differenzierten Darlegung des Reflexionsbegriffs steht der Ausdruck für eine ganze Reihe an Vorstellungen und Strategien, was sich in einer Vielzahl unterschiedlicher Begriffe (z. B. Reflexion, Reflexionsfähigkeit, Reflexivität, Selbstreflexion, usw.) widerspiegelt, die im Kontext des Konstrukts Reflexion in der Literatur zu finden sind und nur unzureichend voneinander abgegrenzt werden (vgl. z. B. Berndt, Häcker \& Leonhard 2017, 11; Lüsebrink \& Grimminger 2014, 204; Slepcevic-Zach et al. 2015, 16; Wyss 2013, 37). In diesem Zusammenhang besteht mit Blick auf den Einsatz von Portfolios im Rahmen der Lehrerbildung ein Handlungsbedarf, denn erst wenn dem Konstrukt Reflexion ausreichend Kontur verliehen wird, kann die Beförderung der Reflexionsfähigkeit im Rahmen von Portfolioarbeit zu voller Entfaltung kommen.

Darüber hinaus plädieren wir dafür, nicht nur den Begriff der Reflexion zu konturieren, sondern darüber hinaus konkrete Hinweise für die Ausgestaltung von Reflexionsanlässen auszuformulieren. Denn trotz einer zunehmenden Forderung nach Einbindung von Reflexionsprozessen in die Lehrerbildung liegt in Bezug auf die Inhalte von Reflexionen sowie die konkrete Formulierung von Reflexionsanlässen zum Auf- und Ausbau von Reflexionsfähigkeit nur unzureichend empirisch hinterlegtes Wissen vor (vgl. Berndt, Häcker \& Leonhard 2017, 11; Roters 2012, 150). Wer also ein Portfolio selbst entwickeln und Reflexionsanlässe und -impulse formulieren möchte, kann auf wenig Bewährtes zurückgreifen. Diesen Umstand greift die hier dargelegte Handlungsempfehlung auf, indem sie dazu auffordert, Fachleiter(inne)n differenziertes und studienseminarspezifisches Material über die Ausgestaltung von Reflexionsanlässen zur Verfügung zu stellen. Damit soll gewährleistet werden, dass die Fachleiter/-innen trotz zeitlich begrenzten Rahmen zumindest in der Vorbereitung der Portfolioarbeit eine Entlastung erfahren.

Eine weitere Handlungsempfehlung liegt darin, Portfolioarbeit stärker in das Arbeitsleben von Fachleiter(inne)n zu integrieren und damit zu einer größeren Selbstverständlichkeit werden zu lassen. Empfehlungen auf der Anwendungsebene können darin liegen, auch zukünftig bei Tagungen verstärkt Portfolios einzusetzen oder im kleineren Kreise, wie z. B. Arbeitsgruppen von Fachleiter(inne)n innerhalb des Studienseminars, verstärkt ein Port- 
folio zu nutzen. $\mathrm{Zu}$ begründen ist diese Handlungsempfehlung damit, dass gelingende Portfolioarbeit mehr als eine Wiederholungsschleife benötigt, um zu gewährleisten, dass die Portfolioarbeit sich einer ständigen Revision unterzieht. Nur so werden Schwachstellen in der Umsetzung der Portfolioarbeit aufgedeckt und Neuerungen aufgenommen.

Abschließend ist festzuhalten, dass die vorangestellten Handlungsempfehlungen sich als erste Konsequenzen der vorangegangen Untersuchung ergeben. Um dezidiertere Erkenntnisse über die Portfolioarbeit in der Lehrerbildung ableiten sowie weitere Handlungsempfehlungen aussprechen zu können, ist es unabdingbar, die empirische Datenbasis auszuweiten. Konkret bedeutet dies, die folgenden Punkte zum Untersuchungsgegenstand zu machen: (1) Untersuchung der Rahmenbedingungen der Portfolioarbeit in allen Phasen der Lehrerbildung, (2) Befragung der beteiligten Akteure an der Portfolioarbeit mit Blick auf gewünschte Hilfestellungen bei der Umsetzung von Portfolioarbeit und (3) Möglichkeiten der Entwicklung und Ausgestaltung zur Unterstützung der Portfolioarbeit in der Lehrerbildung.

\section{Literatur}

BAK (Bundesarbeitskreis der Seminar- und Fachleiter/-innen) (Hrsg.) (2015). Reflektieren und Beraten. URL: http://www.bak-online.de/kongresse/oldenburg/ [24.02.2016].

Berndt, C., Häcker, T. \& Leonhard T. (2017). Editorial. In C. Berndt, T. Häcker \& T. Leonhard (Hrsg.), Reflexive Lehrerbildung revisited: Traditionen - Zugänge Perspektiven (S. 9-18). Bad Heilbrunn: Klinkhardt.

Bräuer, G. (2014). Das Portfolio als Reflexionsmedium für Lehrende und Studierende. Opladen: Budrich.

Dewey, J. (1933). How We Think. Boston: D. C. Heath \& Co.

Döring, N. \& Bortz, J. (2016). Forschungsmethoden und Evaluation in den Sozialund Humanwissenschaften (5. Aufl.). Heidelberg: Springer.

Dubs, R. (2006). Entwicklung von Schlüsselqualifikationen in der Berufsschule. In R. Arnold \& A. Lipsmeier (Hrsg.), Handbuch der Berufsbildung (S. 191-203). Wiesbaden: VS Verlag für Sozialwissenschaften.

Gläser-Zikuda, M. \& Hascher, T. (2007). Zum Potenzial von Lerntagebuch und Portfolio. In M. Gläser-Zikuda \& T. Hascher (Hrsg.), Lernprozesse dokumentieren, refelktieren und beurteilen (S. 9-21). Bad Heilbrunn: Klinkhardt.

Häcker, T. (2005). Portfolio als Instrument der Kompetenzdarstellung und reflexiven Lernprozessteuerung. bwp@, 8, 1-11.

Häcker, T. (2007). Professionalisierung des Lehrerhandelns durch Professional Development Portfolios. Erziehung und Unterricht, 5-6, 382-391

Häcker, T. (2011). Portfolio: Ein Entwicklungsinstrument für selbstbestimmtes Lernen. Eine explorative Studie zur Arbeit mit Portfolios in der Sekundarstufe I (3. Aufl.). Baltmannsweiler: Schneider Verlag Hohengehren. 
Häcker, T. (2012). Portfolioarbeit im Kontext einer reflektierenden Lehrer/innenbildung. In R. Egger \& M. Merkt (Hrsg.), Lernwelt Universität (S. 264-291). Graz: Springer.

Häcker, T. \& Seemann, J. (2013). Von analogen Portfolios für die Entwicklung von digitalen E-Portfolios lernen. In D. Miller \& B. Volk (Hrsg.), E-Portfolio an der Schnittstelle von Studium und Beruf(S. 73-90). München: Waxmann.

Henning, C. \& Kricke, M. (2016). Portfoliodidaktik. Stuttgart: Raabe.

Hertle, E. M. \& Sloane, P. F. E. (2007). Neue Wege in der Berufsschullehrerbildung. In E. M. Hertle \& P. F. E. Sloane (Hrsg.), Portfolio - Kompetenzen - Standards (S. 7-28). Paderborn: Eusl.

Jahncke, H. (2015). Das Portfoliokonzept als Methode zur Beförderung von Selbstreflexionsprozessen von angehenden Lehrerinnen und Lehrern. bwp@, 28, 1-24.

Jahncke, H., Slopinski, A. \& Hüsing, F. (2015). Erfahrungen mit der Portfolioarbeit in Studienseminaren. berufsbildung, 69(151), 39-41.

Koch-Priewe, B. (2013). Das Portfolio in der Lehrerbildung. In B. Koch-Priewe, T. Leonard, A. Pineker \& J. C. Störtländer (Hrsg.), Portfolio in der Lehrerbildung (S. 41-73). Bad Heilbrunn: Klinkhardt.

Kraler, C. (2007). Portfolioarbeit in der LehrerInnenbildung. Eine Standortbestimmung. Erziehung und Unterricht, 5-6, 441-447.

Leonhard, T. (2013). Portfolioarbeit zwischen Reflexion und Leistungsbewertung. In B. Koch-Priewe, T. Leonhard, A. Pineker \& J. C. Strötländer (Hrsg.), Portfolio in der LehrerInnenbildung (S. 180-192). Bad Heilbrunn: Klinkhardt.

Lüsebrink, I. \& Grimminger, E. (2014). Fallorientierte Lehrer- und Lehrerrinnenausbildung evaluieren - Überlegungen zur Modellierung reflexiver Kompetenz. In I. Pieper, P. Frei, K. Hauenschild \& B. Schmidt-Thieme (Hrsg.), Was der Fall ist: Fallarbeit in Bildungsforschung, Lehrerbildung und frühpädagogischen Ausbildungs- und Berufsfeldern (S. 201-212). Wiesbaden: VS Springer.

Neuweg, G. H. (2017). Herrlich unreflektiert. Warum Könner weniger denken, als man denkt. In C. Berndt; T. Häcker \& T. Leonhard (Hrsg.), Reflexive Lehrerbildung revisited: Traditionen - Zugänge - Perspektiven (S. 9-18). Bad Heilbrunn: Klinkhardt.

Paulson, F. L.; Paulson, P. R. \& Meyer, C. A. (1991). What Makes a Portfolio a Portfolio? Educational Leadership, 48(5), 60-63.

Rebmann, K. \& Schlömer, T. (2010). Lehr-Lernarrangements in der Berufsbildung. In K. Büchter (Hrsg.), Enzyklopädie Erziehungswissenschaft Online (EEO), Band „Berufs- und Wirtschaftspädagogik" (S. 1-34). Weinheim: Juventa.

Schön, D. A. (1983). The Reflective Practitioner. New York: Basic Books.

Schwenk, E. (2012). PortfolioPraxis in der Lehrerbildung. Baltmannsweiler: Schneider Verlag Hohengehren.

Slepcevic-Zach, P.; Riebenbauer, E.; Fernandez, K. \& Stock, M. (2015). Einleitung zum eKEP. In P. Slepcevic-Zach, E. Riebenbauer, K. Fernandez \& M. Stock (Hrsg.), eKEP - ein Instrument zur Reflexion und Selbstreflexion (S. 7-19). Graz. Leykam Buchverlagsgesellschaft.

Tettenborn, S. (2014). Portfolioarbeit in der Lehrerbildung. berufsbildung, 42(146), 42-44.

van Dellen, A. (2013). Das Portfolio als Beitrag zur Reflexion persönlicher Kompetenzentwicklung in der Religions-/PädagogInnenbildung. Osterreichisches Religionspädagogisches Forum, 21, 53-61. 
Winter, F. (2007). Was gehört zu guter Portfolioarbeit? Erziehung und Unterricht, 5$6,372-381$.

Winter, F. (2013). Das Portfolio in der Hochschulbildung. In B. Koch-Priewe, T. Leonhard, A. Pineker \& J. C. Strötländer (Hrsg.), Portfolio in der LehrerInnenbildung (S. 15-40). Bad Heilbrunn: Klinkhardt.

Wyss, C. (2013). Unterricht und Reflexion. Eine mehrperspektivische Untersuchungder Unterrichts- und Reflexionskompetenz von Lehrkräften. Münster: Waxmann. 



\title{
Unterrichtliche Überzeugungen und Praktiken von Lehrkräften an Beruflichen Oberschulen - eine fächervergleichende Analyse
}

\author{
Julia Warwas und Andreas Rausch
}

\section{Problemhintergrund und Untersuchungsfeld}

Die Erforschung unterrichtlicher Überzeugungen und Praktiken von Lehrkräften (zsf. Reusser, Pauli \& Elmer 2011; Seidel 2011) beschränkt sich bislang weitgehend auf den allgemeinbildenden Schulsektor. $\mathrm{Zu}$ den wenigen Ausnahmen zählen Studien an Berufsschulen für berufsqualifizierende, duale Ausbildungsgänge (zsf. Seifried 2009; Götzl, Jahn \& Held 2013). Ausgeblendet blieb hingegen das höherqualifizierende, vollzeitschulische Berufsbildungssegment, das im deutschen Schulsystem wichtige Durchlässigkeitsund Berechtigungsfunktionen erfüllt. Berufliche Oberschulen ermöglichen es Personen mit mittlerem Schulabschluss, einen höheren Abschluss mit berufsund arbeitsweltlicher Ausrichtung zu erlangen und damit in den Hochschulsektor einzutreten (z. B. Nikolai \& Ebner 2011). Über diesen ,zweiten Bildungsweg' erwarben im hier betrachteten Bundesland Bayern im Schuljahr 2013/2014 rund 21.000 Schüler/innen die Fachhochschulreife (79\%) oder aber die fachgebundene oder allgemeine Hochschulreife (21\%).

Makrodidaktisch ähnelt die berufliche Oberstufe infolge ihres Höherqualifizierungsauftrags stark der gymnasialen Oberstufe (König 2014): Ziele, thematische Einheiten und Sequenzen des Unterrichts richten sich - anders als in der dualen Berufsausbildung - nicht an arbeitsprozessnah strukturierten Lernfeldern, sondern an fachinhaltlich sortierten Lehrplänen aus. Damit steht der Unterricht an Beruflichen Oberschulen der Wissenssystematik akademischer Bezugsdisziplinen näher als der situativen Handlungslogik spezifischer Berufsgruppen (Reetz \& Seyd 2006). Dennoch ist sowohl in den sog. Profilfächern der gängigen Ausbildungsrichtungen „Technik“, „Wirtschaft und Verwaltung“, „Sozialwesen“" sowie „Agrarwirtschaft, Bio- und Umwelttechnologie" als auch in allgemeinbildenden Fächern intendiert, arbeitspraktische Anforderungen in relevanten Berufsfeldern erkennbar im Unterricht aufzugreifen (Pahl 2014; ISB o.J.).

Vor diesem Hintergrund stellt sich die Frage, welche Vorstellungen Lehrkräfte an Beruflichen Oberschulen über erfolgreiche Lehr-Lern-Prozesse hegen (unterrichtliche Überzeugungen), wie sie diese Prozesse gewöhnlich gestalten (unterrichtliche Praktiken) und inwieweit es ihnen dabei gelingt, 
Bezüge zur jeweils berufsfeldtypischen Arbeitspraxis herzustellen. Die fächerbasierte Strukturierung der Curricula veranlasst dabei ebenso wie das Fehlen einer schulformspezifischen Mikrodidaktik (Pahl 2014) zur Annahme, dass jene Überzeugungen und Praktiken unterschiedliche Fachkulturen widerspiegeln. Im vorliegenden Beitrag möchten wir erste Schritte zur empirischen Untersuchung dieser Annahme unternehmen, indem wir anhand einer Fragebogenerhebung unter Lehrkräften und Schüler/innen an Beruflichen Oberschulen in Bayern fachbereichsabhängige Unterschiede des Unterrichtens unter Kontrolle konkurrierender Einflussfaktoren überprüfen.

\section{Unterrichtliche Überzeugungen und Praktiken aus fachkultureller Perspektive}

Aus dem Blickwinkel der Fachkulturforschung konstituieren die in Lehrplänen abgegrenzten Fächer nicht lediglich stabile Formate der Lernziel- und Wissensorganisation (Tenorth 1999; Lüders 2007). Sie prägen auch nachhaltig den Habitus der jeweiligen Fachvertreter/innen, in welchem sich geteilte „Wahrnehmungs-, Denk-, Bewertungs- und Handlungsmuster“" (Huber 1991, 422) als modus operandi der pädagogischen Betätigung widerspiegeln. Umgekehrt sorgt der gewohnheitsmäßige Rückgriff auf derartige Muster langfristig dafür, dass Fachkulturen entstehen und die hier geltenden „Gemeinsamkeiten im denkenden, fragenden, forschenden und lehrenden Umgang mit relevanten Ausschnitten der Wirklichkeit" (Hericks \& Körber 2007, 31) als opus operatum des Unterrichtsalltags stetig reproduzieren (Müller-Roselius 2007).

Gemäß der Inkorporationsannahme entwickelt und festigt sich der Fachlehrendenhabitus durch Sozialisationsprozesse. Hierzu gehören eigene Schulerfahrungen, vor allem aber eine disziplinorientierte universitäre Lehrerbildung mit hohen fachwissenschaftlichen und fachdidaktischen Anteilen (Willems 2007). Gemäß der Unbewusstheitsannahme leiten die erworbenen Denk-, Wertungs- und Handlungsmuster didaktische Entwürfe wie auch deren Realisierungen nahezu intuitiv an und lassen den FachlehrendenHabitus als „Stilgenerator“" wirken (Willems 2008). Unter dem Handlungsdruck laufender Interaktionen im Klassenzimmer erlaubt er prompte Reaktionen, die jedoch schematisch ausfallen und folglich auch schülerseitige Erwartungen an charakteristische Choreografien etwa einer Mathematikstunde entstehen lassen und wiederkehrend bestätigen (Lüders 2007). So dokumentieren bspw. Beobachtungen im Rechnungswesen-Unterricht an dualen Berufsschulen eine Dominanz frontaler, lehrerzentrierter Unterrichtsmethoden, welche sich nach Auffassung der ergänzend befragten Lehrpersonen für diese Inhalte auch besser eignen als schüleraktive, handlungsorientierte Methoden 
(z. B. Götzl, Jahn \& Held 2013). Korrespondierend hiermit berichten Berufsschüler/innen davon, einen Großteil der Unterrichtszeit für das Abschreiben von der Tafel, das Memorieren von Regeln und Verfahren sowie das mechanische Üben von Buchungssätzen und Rechenoperationen zu verwenden (Seifried 2009). Der Fachkulturansatz charakterisiert daher auch Entscheidungen zwischen didaktischen Handlungsalternativen als habituell überformt, d. h. als eine fachlich gebändigte pädagogische Autonomie, die Gestaltungsspielräume stets nur innerhalb der Grenzen fachtypischer Aufgabenzuschnitte, (Inter-)Aktionsformen und Methoden ausschöpft (Müller-Roselius 2007). Der normierende Einsatz gleicher Lehrbücher und -materialien sowie die regulierende Kraft innerschulischer Fachbereichsgremien (Clement 2006) tragen zusätzlich dazu bei, dass sich fachintern Vorstellungen von gutem Unterricht annähern und gelebte Unterrichtstraditionen verstetigen. Widerstände gegen curriculare und didaktische Reformen zeugen im Sinne der Stabilitätsannahme von der Veränderungsresistenz etablierter Fachkulturen (z. B. Terhart 2013).

\section{Unterrichtliche Überzeugungen und Praktiken im Spiegel der Lehr-Lern-Forschung}

Die empirische Lehr-Lern-Forschung (zsf. Achtenhagen \& Pätzold 2010 für berufliche Schulen) betrachtet den (Fach-)Inhalt traditionsgemäß als einen von vielen Bestimmungsgründen der Sicht- und Handlungsweisen von Lehrkräften. Überblickswerke betonen dabei den Einfluss sog. internaler und externaler Bedingungen der pädagogischen Betätigung (z. B. Cross Francis, Rapacki \& Eker 2015).

$\mathrm{Zu}$ den folgenreichsten externalen (bzw. kontextspezifischen) Bedingungsfaktoren zählen gemäß Buehl und Beck (2015) die vorhandenen bzw. subjektiv wahrgenommenen Fähigkeiten, Einstellungen und Verhaltensweisen des zu unterrichtenden Schülerklientels. Korrespondierend hiermit führt Mansour (2009) aus, dass Disziplinprobleme, aber auch zeitliche Restriktionen und große Klassen kontextabhängige Zwänge darstellen, welche insbes. die Einsatzhäufigkeit lehrerzentrierter Sozialformen und repetitiver Übungsphasen erhöhen.

Im Bereich internaler (bzw. personenspezifischer) Bedingungsfaktoren gelten Selbstwirksamkeitsüberzeugungen der Lehrkräfte als robuste Prädiktoren ihrer Sicht- und Handlungsweisen (Klassen \& Tze 2014). Woolfolk Hoy, Hoy und Davis (2009) schließen aus den von ihnen gesichteten Studien, dass hoch selbstwirksame Lehrkräfte verglichen mit gering selbstwirksamen Kolleg/innen eine größere und auch bei Rückschlägen anhaltende Bereitschaft zu methodischen Variationen und zum Einsatz schülerzentrierter Sozi- 
alformen aufweisen, den Unterrichtsverlauf flexibler an die Bedürfnisse der Lernenden anpassen, ihnen mehr Wahlfreiheiten bei der Aufgabenbearbeitung zugestehen und auf Verständnisprobleme weniger mit Kritik als mit Beratung und Begleitung reagieren. Beobachtungsstudien im Berufsschulunterricht legen außerdem nahe, dass erfahrene Lehrkräfte häufiger frontale Methoden einsetzen als Novizen (Götzl, Jahn \& Held 2013).

\section{Fragestellungen, Analysegegenstände und Kontrollvariablen der vorliegenden Studie}

Für die Analyse fachkulturell geprägter Sicht- und Handlungsweisen ergeben sich aus den vorangegangenen Überlegungen zwei Leitfragen:

1. Unterscheiden sich Vertreter/innen verschiedener Fächergruppen hinsichtlich ihrer unterrichtlichen Überzeugungen, wenn die Effekte internaler und externaler Bedingungen der pädagogischen Betätigung kontrolliert werden?

2. Lassen sich von Schüler/innen wahrgenommene Merkmale der Unterrichtsgestaltung auf die Fachzugehörigkeit der Lehrkräfte zurückführen, wenn die Effekte internaler und externaler Bedingungen der pädagogischen Betätigung, aber auch die Effekte lehrerindividueller Überzeugungen kontrolliert werden?

Unterrichtliche Überzeugungen charakterisieren wir dabei anhand der subjektiven Bevorzugung didaktischer Strategien, die eher transmissive oder eher konstruktivistische Grundideen beim Lehren und Lernen fachspezifischer Unterrichtsinhalte realisieren (Kuntze 2012). So kann etwa die Strategie des repetitiven und prüfungsorientierten Einübens fachspezifischer Arbeitstechniken i.S.v. Drill and Practice (Lim, Tang \& Kor 2012) als Ausdruck einer transmissiven Grundorientierung gewertet werden, bei welcher die Lehrkraft bestrebt ist, Lerninhalte kleinschrittig darzubieten und Schüler/innen mit korrekten Lösungsalgorithmen für klar zugeschnittene Aufgabenformate auszustatten. Demgegenüber stellen die Bearbeitung authentischer Probleme aus dem beruflichen Alltag (z. B. Sembill, Wuttke, Seifried, Egloffstein \& Rausch 2007), das eigenständige Entdecken und Ausloten von Lösungsstrategien (z. B. Winter 2016) oder das Diskutieren und Argumentieren mit Klassenkamerad/innen (z. B. Wuttke 2012) beispielhafte konstruktivistische Strategien dar, die Schüler/innen dazu anregen sollen, fachspezifische Wissensstrukturen und Arbeitsweisen anknüpfend an ihren bisherigen Erfahrungshorizont (inter)aktiv zu erschließen. 
Unterrichtliche Praktiken werden anhand von Merkmalen der Ausführung, Regulation und Organisation von Lernaktivitäten ausdifferenziert (Seidel 2011) und mittels schülerseitiger Wahrnehmungen des von den befragten Lehrkräften erteilten Fachunterrichts operationalisiert (z. B. Grühn 2000). Im Bereich der Ausführung unterrichtlicher Lernaktivitäten betrachten wir das Ausmaß, in dem Schüler/innen die Auseinandersetzung mit den Fachinhalten erstens als kognitiv herausfordernd und zweitens als arbeitsweltlichanwendungsbezogen wahrnehmen (s. auch Seeber \& Squarra 2003). Bei der Regulationskomponente konzentrieren wir uns auf das Erleben einer anregenden und unterstützenden Lernumgebung. Die Organisation von Lernaktivitäten wird anhand der wahrgenommenen Einsatzhäufigkeit verschiedener Sozialformen abgebildet.

Als Kontrollvariablen, deren jeweilige Effekte bei der Ermittlung fachgebundener Differenzen rechnerisch herauspartialisiert werden, fließen entsprechend der verfügbaren Meta-Analysen und Forschungsübersichten berufliche Selbstwirksamkeitsüberzeugungen der Lehrkräfte, lernrelevante Voraussetzungen der Schüler/innen, ein gelingendes Klassenmanagement sowie die Größe der unterrichteten Klasse ein. Zudem berücksichtigt die vorliegende Studie das Geschlecht der Lehrkräfte, die Höhe ihres Lehrdeputats sowie als Indikator beruflicher Erfahrung - ihr Dienstalter.

Die Untersuchung der genannten Leitfragen erscheint nicht nur deshalb lohnend, weil Berufliche Oberschulen, wie eingangs erwähnt, trotz ihrer spezifischen makrodidaktischen Struktur bisher in der empirischen Berufsbildungsforschung unbeachtet blieben. Sie gewinnt angesichts der in Kapitel 2 erläuterten Veränderungsresistenz etablierter Fachkulturen und aktuell geplanter curricularer Reformen (siehe hierzu die laufenden Veröffentlichungen auf den Interentseiten des ISB Bayern) zusätzlich an Bedeutung.

\section{Methode}

\subsection{Stichprobe und Instrumentierung}

An sieben Schulen füllten 550 Schüler/innen aus 29 Klassen geschlossene Fragebögen mit Ratingskalen und persönlichen Angaben aus. 46.2\% der Teilnehmenden sind männlich; die große Mehrheit befindet sich in den Altersklassen 17-18 Jahre (45.5\%) sowie über 19 Jahre (49.0\%). Anhand lehrerseitiger Angaben zum erteilten Unterricht ergibt sich folgende Einteilung: mathematisch-naturwissenschaftliche (MN-) Fächer $=5$ Klassen; wirtschaftswissenschaftliche (W-) Fächer $=7$ Klassen; technologisch-technische (T-) Fächer $=7$ Klassen; sprachliche (S-) Fächer $=5$ Klassen; erziehungsund sozialwissenschaftliche (ES-) Fächer $=5$ Klassen. Das männliche Lehr- 
personal (insgesamt 16 Personen) dominiert in den MN-Fächern $(4 \mathrm{~m} / 1 \mathrm{w})$ und T-Fächern $(6 \mathrm{~m} / 1 \mathrm{w})$. Breit streuen das Ausmaß der Berufserfahrung (gemessen an der Unterrichtstätigkeit in Jahren: $\mathrm{M}=16.31 ; \mathrm{SD}=9.11$; $\mathrm{Min}=2$; Max = 30), der Umfang der Lehrverpflichtung (in Wochenstunden: $\mathrm{M}=$ 19.62; $\mathrm{SD}=5.73$; $\mathrm{Min}=6$; $\mathrm{Max}=26$ ) sowie die Größe der unterrichteten Klasse $(M=20$ Schüler/innen; $\mathrm{SD}=5.00 ; \mathrm{Min}=10 ; \mathrm{Max}=30)$.

Tabelle 1 dokumentiert die deskriptiven Kennwerte der aus Schülersicht erfassten unterrichtlichen Praktiken. Ihnen ist zu entnehmen, dass zwar innerhalb jeder Klassengemeinschaft weitgehende Einigkeit hinsichtlich aller $\mathrm{zu}$ bewertenden Dimensionen der Unterrichtsgestaltung besteht (M(AD)Werte $\leq 1.00)$, markante Abweichungen zwischen den Fachlehrkräften jedoch vorrangig im Bereich der Ausführung und Regulation von Lernaktivitäten erlebt werden $\left(\mathrm{ICC}_{1}\right.$-Werte $\left.\geq 10 \%\right)$. Bei der Organisation von Lernaktivitäten finden sich hingegen klassenübergreifend hohe Anteile lehrerzentrierter Sozialformen, v.a. das gelenkte Lehrgespräch.

Tab. 1: Deskriptive Kennwerte der schülerseitigen Wahrnehmungen unterrichtlicher Praxis

\begin{tabular}{|c|c|c|c|c|c|c|}
\hline Konstrukt (Itemzahl) & $\mathrm{M}$ & SD & Alpha & $\mathrm{ICC}_{1}$ & $\mathrm{ICC}_{2}$ & $\mathrm{M}(\mathrm{AD})$ \\
\hline \multicolumn{7}{|c|}{ Ausführung und Regulation von Lernaktivitäten } \\
\hline Anforderungsniveau der & $3.96^{\mathrm{a}}$ & 1.09 & .78 & .17 & .80 & .77 \\
\hline \multicolumn{7}{|l|}{ Unterrichtsinhalte (3) } \\
\hline Anwendungsbezug der & $3.75^{\mathrm{a}}$ & 1.30 & .87 & .20 & .83 & .93 \\
\hline \multicolumn{7}{|l|}{ Unterrichtsinhalte (3) } \\
\hline Anregende, unterstützende & $3.96^{\mathrm{a}}$ & 1.09 & .89 & .34 & .91 & .69 \\
\hline Unterrichtsgestaltung (6) & & & & & & \\
\hline \multicolumn{7}{|c|}{ Organisation von Lernaktivitäten (Sozialformen) } \\
\hline Lehrvortrag (1) & $4.47^{b}$ & 1.13 & --- & .05 & .51 & .82 \\
\hline Lehrgespräch (1) & $4.90^{\mathrm{b}}$ & .88 & --- & .01 & .19 & .59 \\
\hline Einzelarbeit (1) & $4.48^{\mathrm{b}}$ & 1.01 & --- & .07 & .61 & .73 \\
\hline Partnerarbeit (1) & $4.40^{\mathrm{b}}$ & .89 & --- & .06 & .55 & .67 \\
\hline Problemzentrierte & $3.71^{\mathrm{b}}$ & 1.17 & --- & .12 & .75 & .84 \\
\hline \multicolumn{7}{|l|}{ Gruppenarbeit (1) } \\
\hline
\end{tabular}

Hinweise: Antwortformate von $1=$ stimmt gar nicht bis $6=$ stimmt voll und ganz (a) sowie von $1=$ nie bis $6=$ immer $(\mathrm{b})$. Alpha $=$ Cronbachs Alpha; ICC $=$ Intraclass correlation; $\mathrm{M}(\mathrm{AD})=$ Mean Absolute Deviation

Quelle: Eigene Darstellung

Lehrkräfte schätzten eigene Fähigkeiten zur Bewältigung beruflicher Anforderungen (berufliche Selbstwirksamkeit: $\mathrm{M}=4.57$; $\mathrm{SD}=.78 ; \alpha=.86$ ) sowie zur Sicherstellung einer störungsarmen, geordneten Lernumgebung in der unterrichteten Klasse (gelingendes Klassenmanagement: $\mathrm{M}=4.69 ; \mathrm{SD}=$ $1.02 ; \alpha=.89)$ auf sechsfach gestuften Antwortformaten ein. Anhand bipolarer Adjektivlisten beurteilten sie zudem, inwiefern die Schüler/innen der 
unterrichteten Klasse über günstige bzw. ungünstige Eigenschaften für erfolgreiche Lernprozesse verfügen (z. B. ziellos versus zielstrebig), wobei hohe Ausprägungen der zugehörigen Skala lernförderliche Voraussetzungen indizieren $(\mathrm{M}=3.99 ; \mathrm{SD}=.89 ; \alpha=.88)$. Darüber hinaus signalisierten die Lehrkräfte mithilfe sechsfach gestufter Antwortformate ihre Zustimmung zu beschreibenden Aussagen didaktischer Strategien: Diskursive und anwendungsorientierte Auseinandersetzung $(\mathrm{M}=4.53$; $\mathrm{SD}=1.14 ; \alpha=.89)$, Entdeckendes Vorgehen und innere Differenzierung $(\mathrm{M}=3.74 ; \mathrm{SD}=1.06 ; \alpha=.72)$ sowie Anleiten und Einüben $(\mathrm{M}=4.26 ; \mathrm{SD}=1.32 ; \alpha=.72)$.

\subsection{Vorbereitende Analysen und verwendete Effektstärkenmaße}

Die Auswahl von Kontrollvariablen für die Kovarianzanalysen erfolgte anhand bivariater Zusammenhänge mit den Kriteriumsvariablen, wobei aufgrund der geringen Klassenzahl die Korrelationsstärke das Selektionskriterium darstellte. Als Schwellenwert wurde ein mindestens moderater Zusammenhang in Höhe von $r \geq .30$ zugrunde gelegt (Cohen 1992). Demzufolge bestehen systematische positive Beziehungen

- zwischen der Überzeugung Diskurs und Anwendung und einem gelingendem Klassenmanagement $(r=.36)$ sowie zwischen der Überzeugung Anleiten und Einüben und dem Lehrdeputat in Wochenstunden $(\mathrm{r}=.32)$;

- zwischen schülerseitg wahrgenommener Anregung und Unterstützung und beruflicher Selbstwirksamkeit der Lehrkraft $(\mathrm{r}=.40)$ wie auch einem gelingenden Klassenmanagement $(\mathrm{r}=.40)$ sowie zwischen dem schülerseitig wahrgenommenen Anwendungsbezug und einem gelingenden Klassenmanagement $(\mathrm{r}=.57)$ wie auch der lehrerindividuellen Überzeugung Diskurs und Anwendung $(\mathrm{r}=.33)$;

- zwischen der schülerseitig wahrgenommenen Einsatzhäufigkeit von Partnerarbeit und dem Geschlecht der Lehrkraft $(\mathrm{r}=.30$, wobei $0=$ männlich und 1 = weiblich).

Ferner existieren negative Zusammenhänge

- zwischen der Überzeugung Anleiten und Einüben und gelingendem Klassenmanagement $(\mathrm{r}=-.30)$;

- zwischen schülerseitg wahrgenommener Anregung und Unterstützung und der Klassengröße $(\mathrm{r}=-.37)$;

- $\quad$ zwischen der schülerseitig wahrgenommenen Einsatzhäufigkeit von Einzelarbeit und und der lehrerindividuellen Überzeugung Diskurs und Anwendung $(\mathrm{r}=-.34)$ wie auch den lehrerseitig eingeschätzten lernförderlichen Voraussetzungen der Schüler/innen $(r=-.30)$. 
In den nachfolgenden Kovarianzanalysen wird das Ausmaß fachgebundener Unterschiede in unterrichtlichen Überzeugungen und Praktiken anhand von adjustierten f-Werten quantifiziert, um einer Überschätzung der praktischen Bedeutsamkeit von Mittelwertdifferenzen in kleinen Stichproben entgegenzuwirken. Für die metrischen Prädiktoren wird das ebenfalls konservative Effektstärkenmaß $\varepsilon^{2}$ verwendet. Die Interpretation folgt der Klassifikation für f- und $\eta^{2}$-Werte nach Cohen (1992) und ermöglicht eine Abgrenzung von kleinen Effekten $\left(f_{\text {adj }} \geq .10\right.$ bzw. $\left.\varepsilon^{2} \geq .01\right)$, mittleren Effekten $\left(f_{\text {adj }} \geq .25\right.$ bzw. $\varepsilon^{2} \geq .06$ ) und großen Effekte ( $f_{\text {adj }} \geq .40$ bzw. $\varepsilon^{2} \geq .14$ ). Für eine zufallskritische Absicherung der Ergebnisse wird aufgrund der geringen Zahl der Vergleichsgruppen ein Alpha-Fehlerniveau $\leq .10$ akzeptiert.

\section{Empirische Befunde}

Entsprechend Leitfrage 1 werden zunächst Einflüsse des Fachs auf unterrichtliche Überzeugungen überprüft. Tabelle 2 dokumentiert für die Strategie Diskurs und Anwendung substanzielle Abweichungen mittleren Ausmaßes zwischen den betrachteten Fächergruppen der Stichprobe. Auch unter Kontrolle subjektiver Einschätzungen eines gelingenden Klassenmanagements, die fachübergreifend positiv mit dieser Strategie verknüpft sind ( $F=3.046$; B $=.38 ; \mathrm{SE}=.22 ; \varepsilon^{2}=.09$ ), sprechen sich S- und ES-Lehrkräfte überzufällig stärker für diskursive und anwendungsbezogene Lehrstrategien aus als MNLehrkräfte. S-Lehrkräfte mit den höchsten Ausprägungen weichen zudem deutlich von W-Lehrkräften ab.

Signifikante und im Umfang erhebliche fachabhängige Variationen treten bei der Strategie Entdecken und Differenzieren zutage. W-Lehrkräfte verfolgen diese Strategie nach eigenen Angaben deutlich weniger als Kolleg/innen in allen anderen Fachbereichen. Unter den S-Lehrkräften sind erneut die höchsten Zustimmungswerte zu verzeichnen.

Hinsichtlich der Strategie Anleiten und Einüben unterscheiden sich die Lehrkräfte verschiedener Fachgruppen jedoch kaum. Lediglich W-Lehrkräfte weichen signifikant von T-Lehrkräften ab und erreichen die nominell höchsten Zustimmungswerte auf dieser Skala. Darüber hinaus erweist sich die Höhe der individuellen Unterrichtsverpflichtung fachübergreifend als eigenständiger Prädiktor $\left(\mathrm{F}=4.001 ; \mathrm{B}=.58 ; \mathrm{SE}=.29 ; \varepsilon^{2}=.13\right)$. An Beruflichen Oberschulen räumen Lehrkräfte dem ausführlichen Anleiten und wiederholenden Einüben von Arbeitsschritten demnach einen umso größeren Stellenwert im Unterricht ein, je mehr Wochenstunden sie erteilen. 
Tab. 2: (Adjustierte) Mittelwerte, Standardfehler und (ko-)varianzanalytische Ergebnisse für fachabhängige Variationen unterrichtlicher Überzeugungen

\begin{tabular}{|c|c|c|c|c|c|c|c|}
\hline \multirow[b]{2}{*}{ Konstrukt } & \multicolumn{5}{|c|}{$\begin{array}{l}\text { Deskriptive Kennwerte der Fachbereiche: } \\
\qquad \text { M (SE) }\end{array}$} & \multicolumn{2}{|c|}{$\mathrm{AN}(\mathrm{C}) \mathrm{OVA}$} \\
\hline & $\begin{array}{c}\text { S- } \\
\text { Fächer }\end{array}$ & $\begin{array}{c}\text { W- } \\
\text { Fächer }\end{array}$ & $\begin{array}{c}\text { T- } \\
\text { Fächer }\end{array}$ & $\begin{array}{c}\text { ES- } \\
\text { Fächer }\end{array}$ & $\begin{array}{l}\text { MN- } \\
\text { Fächer }\end{array}$ & $\mathrm{F}$ & $f_{\text {adj. }}$. \\
\hline $\begin{array}{l}\text { Diskurs und } \\
\text { Anwendung }\end{array}$ & $\begin{array}{l}5.24 \\
(.47)\end{array}$ & $\begin{array}{l}4.06 \\
(.40)\end{array}$ & $\begin{aligned} & 4.71 \\
&(.38) \\
&> \mathrm{W}^{+}, \mathrm{M} \\
& \mathrm{E}>\mathrm{MN}\end{aligned}$ & $\begin{array}{l}5.01 \\
(.49)\end{array}$ & $\begin{array}{l}3.78 \\
(.46)\end{array}$ & 1.978 & .37 \\
\hline $\begin{array}{l}\text { Entdecken und } \\
\text { Differenzieren }\end{array}$ & $\begin{array}{l}4.93 \\
(.35)\end{array}$ & $\begin{array}{c}2.62 \\
(.29) \\
S>V \\
W \\
\end{array}$ & $\begin{array}{r}3.91 \\
(.29) \\
\mathrm{T}^{*}, \mathrm{ES} \\
* *, \mathrm{ES}^{+},\end{array}$ & $\begin{array}{r}3.53 \\
(.35) \\
\mathrm{MN}^{+} \\
\mathrm{N}^{* *}\end{array}$ & $\begin{array}{l}4.07 \\
(.35)\end{array}$ & $6.942 * *$ & .91 \\
\hline $\begin{array}{l}\text { Anleiten und } \\
\text { Einüben }\end{array}$ & $\begin{array}{l}4.20 \\
(.57)\end{array}$ & $\begin{array}{l}4.75 \\
(.47)\end{array}$ & $\begin{array}{c}3.33 \\
(.49) \\
\mathrm{W}>\mathrm{T}^{*}\end{array}$ & $\begin{array}{l}4.56 \\
(.56)\end{array}$ & $\begin{array}{l}4.64 \\
(.67)\end{array}$ & 1.318 & .21 \\
\hline
\end{tabular}

Hinweise:

$\mathrm{M}=$ Mittelwert; SE = Standardfehler der Schätzung; Antwortformat von 1 (trifft gar nicht zu) bis 6 (trifft sehr zu)

$\mathrm{S}=$ sprachliche, $\mathrm{W}=$ wirtschaftswissenschaftliche, $\mathrm{T}=$ technologisch-technische, $\mathrm{ES}=$

erziehungs- und sozialwissenschaftliche, $\mathrm{MN}$ = mathematisch-naturwissenschaftliche Fächer

$+\mathrm{p} \leq 0.10 ; * \mathrm{p} \leq .05 ; * * \mathrm{p} \leq .01$

Quelle: Eigene Darstellung

Zur Beantwortung der zweiten Forschungsfrage berichtet Tabelle 3 (adjustierte) Mittelwerte und (ko-)varianzanalytische Ergebnisse für die schülerseitig wahrgenommenen Merkmale der Unterrichtsgestaltung. In der Dimension Anregung und Unterstützung weichen allerdings bei simultaner Schätzung internaler und externaler Bedingungsfaktoren nur W-Lehrkräfte systematisch von ES-Lehrkräften ab, wobei Erstere die niedrigsten, Letztere die höchsten Bewertungen erhalten. Die berücksichtigten Kovariaten wirken dabei additiv, ohne dass sich ein signifikanter Einzelprädiktor ausmachen ließe. Die größte Varianzbindung erzielen die beruflichen Selbstwirksamkeitsüberzeugungen der unterrichtenden Lehrkraft $\left(\mathrm{F}=2.691 ; \mathrm{B}=.20 ; \mathrm{SE}=.12 ; \varepsilon^{2}=.07\right)$.

Fachgebundene Unterschiede im Anwendungsbezug der Unterichtsinhalte zeigen sich in der Stichprobe in mittlerem Ausmaß. Erneut sind es die ESLehrkräfte, denen eine arbeitsplatznahe Auseinandersetzung mit Lerninhalten bescheinigt wird. Hierin unterscheiden sie sich nicht nur von W-Lehrkräften, sondern auch von MN- und S-Lehrkräften überzufällig. Fachübergreifend erweist sich zudem ein effizientes Klassenmanagement als prognostisch hoch bedeutsam für einen anwendungsorientierten Unterricht $(\mathrm{F}=5.981 ; \mathrm{B}=.28$; $\left.\mathrm{SE}=.11 ; \varepsilon^{2}=.13\right)$. Das Anforderungsniveau der Lerninhalte empfinden Schüler/innen hingegen in allen Fachbereichen als gleichwertig. 
Fachabhängige Schwankungen beim Einsatz von Partnerarbeit sowie Einzelarbeit lassen sich bei gleichzeitiger Berücksichtigung von Kontrollvariablen nicht bzw. nur in geringem Umfang feststellen. Stattdessen zeigt sich, dass Lehrkräfte, welche von der Wirksamkeit einer diskursiven und anwendungsorientierten Auseinandersetzung mit Lerninhalten überzeugt sind, ungeachtet ihrer Fachzugehörigkeit deutlich seltener Aufgaben in Einzelarbeit bearbeiten lassen als Lehrkräfte, die diese Auffassung weniger teilen ( $\mathrm{F}=$ 3.056; $\mathrm{B}=-.11 ; \mathrm{SE}=.06 ; \varepsilon^{2}=.09$ ). Zudem neigen weibliche Lehrkräfte häufiger als ihre männlichen Kollegen dazu, in schüleraktiven Phasen jeweils Banknachbar/innen zusammenarbeiten zu lassen $(\mathrm{F}=2.312 ; \mathrm{B}=-.19$; $\mathrm{SE}=$ $\left..13 ; \varepsilon^{2}=.08\right)$.

Tab. 3: (Adjustierte) Mittelwerte, Standardfehler und (ko-)varianzanalytische Ergebnisse für fachabhängige Variationen unterrichtlicher Praktiken

\begin{tabular}{|c|c|c|c|c|c|c|c|}
\hline \multirow[b]{2}{*}{ Konstrukt } & \multicolumn{5}{|c|}{$\begin{array}{l}\text { Deskriptive Kennwerte der Fachbereiche: } \\
\text { M (SE) }\end{array}$} & \multicolumn{2}{|c|}{$\mathrm{AN}(\mathrm{C}) \mathrm{OVA}$} \\
\hline & $\begin{array}{c}\text { S- } \\
\text { Fächer }\end{array}$ & $\begin{array}{c}\text { W- } \\
\text { Fächer }\end{array}$ & $\begin{array}{c}\text { T- } \\
\text { Fächer }\end{array}$ & $\begin{array}{c}\text { ES- } \\
\text { Fächer }\end{array}$ & $\begin{array}{c}\text { MN- } \\
\text { Fächer }\end{array}$ & $\mathrm{F}$ & $f_{\text {adj. }}$ \\
\hline \multicolumn{8}{|c|}{ Ausführung und Regulation von Lernaktivitäten } \\
\hline $\begin{array}{l}\text { Anregung und } \\
\text { Unterstützung }\end{array}$ & $\begin{array}{l}4.08 \\
(.31)\end{array}$ & $\begin{array}{l}3.66 \\
(.23)\end{array}$ & $\begin{array}{c}4.14 \\
(.23) \\
\mathrm{ES}>\mathrm{W}^{*} \\
\end{array}$ & $\begin{array}{l}4.36 \\
(.31)\end{array}$ & $\begin{array}{l}4.01 \\
(.29)\end{array}$ & 1.033 & .07 \\
\hline Anwendungsbezug & $\begin{array}{l}3.74 \\
(.24)\end{array}$ & $\begin{array}{l}3.62 \\
(.20) \\
\mathrm{ES}\end{array}$ & $\begin{array}{r}3.87 \\
(.19) \\
\mathrm{S}^{+}, \mathrm{W}^{*},\end{array}$ & $\begin{array}{l}4.37 \\
(.25) \\
1 N^{*}\end{array}$ & $\begin{array}{l}3.41 \\
(.24)\end{array}$ & 2.126 & .39 \\
\hline Anforderungsniveau & $\begin{array}{l}2.98 \\
(.22) \\
\end{array}$ & $\begin{array}{l}3.31 \\
(.19) \\
\end{array}$ & $\begin{array}{l}2.96 \\
(.19) \\
\end{array}$ & $\begin{array}{l}3.22 \\
(.22) \\
\end{array}$ & $\begin{array}{l}3.31 \\
(.22) \\
\end{array}$ & .763 & .00 \\
\hline \multicolumn{8}{|c|}{ Organisation von Lernaktivitäten (Sozialformen) } \\
\hline Einzelarbeit & $\begin{array}{l}4.47 \\
(.15)\end{array}$ & $\begin{array}{l}4.45 \\
(.13)\end{array}$ & $\begin{array}{l}4.70 \\
(.14)\end{array}$ & $\begin{array}{l}4.29 \\
(.17)\end{array}$ & $\begin{array}{l}4.27 \\
(.16)\end{array}$ & 1.266 & .19 \\
\hline \multicolumn{8}{|c|}{$\mathrm{T}>\mathrm{MN}^{+}, \mathrm{ES}^{+}$} \\
\hline Partnerarbeit & $\begin{array}{l}4.32 \\
(.14)\end{array}$ & $\begin{array}{l}4.35 \\
(.12) \\
\end{array}$ & $\begin{array}{l}4.50 \\
(.12)\end{array}$ & $\begin{array}{l}4.60 \\
(.14)\end{array}$ & $\begin{array}{l}4.35 \\
(.14)\end{array}$ & .799 & .00 \\
\hline $\begin{array}{l}\text { Problemzentrierte } \\
\text { Gruppenarbeit }\end{array}$ & $\begin{array}{l}3.54 \\
(.22)\end{array}$ & $\begin{array}{l}3.87 \\
(.19)\end{array}$ & $\begin{array}{l}3.63 \\
(.19)\end{array}$ & $\begin{array}{l}4.08 \\
(.22)\end{array}$ & $\begin{array}{l}3.53 \\
(.22)\end{array}$ & 1.224 & .18 \\
\hline
\end{tabular}

Hinweise:

$\mathrm{M}=$ Mittelwert; $\mathrm{SE}=$ Standardfehler der Schätzung

Antwortformat von $1=$ stimmt gar nicht bis $6=$ stimmt voll und ganz (Ausführung und Regulation) bzw. von $1=$ fast nie bis $6=$ fast immer (Organisation)

$\mathrm{S}=$ sprachliche, $\mathrm{W}=$ wirtschaftswissenschaftliche, $\mathrm{T}=$ technologisch-technische, $\mathrm{ES}=$ erziehungs- und sozialwissenschaftliche, $\mathrm{MN}=$ mathematisch-naturwissenschaftliche Fächer $+\mathrm{p} \leq 0.10 ; * \mathrm{p} \leq .05 ; * * \mathrm{p} \leq .01$

Quelle: Eigene Darstellung 
Die Häufigkeit, in der problemzentrierte Gruppenarbeiten durchgeführt werden, differiert wiederum nur in geringem Ausmaß zwischen den Fachbereichen. Diese Unterschiede lassen sich vorrangig darauf zurückführen, dass ES-Fächer höhere Merkmalsausprägungen aufweisen als S- und MN-Fächer.

\section{Zusammenfassung und Ausblick}

Wir sind der Frage nachgegangen, ob und in welcher Hinsicht unterrichtsbezogene Überzeugungen und Praktiken von Lehrkräften an Beruflichen Oberschulen fachkulturell geprägt sind. Anhand der Befragungsdaten von 550 Schüler/innen aus 29 Klassen und ihren Lehrkräften lassen sich diese Überzeugungen und Praktiken erstmals näher charakterisieren, auch wenn sie zweifellos unter dem Vorbehalt fehlender Generalisierbarkeit angesichts der geringen Klassenzahl stehen. Aus demselben Grund mussten die Fachbereiche in breit gefasste Kategorien unterteilt werden (bspw. wirtschaftswissenschaftliche Fächer), wodurch sicherlich bestehende Binnenvariationen (etwa zwischen Betriebs- und Volkswirtschaftslehre) vernachlässigt werden. Diesen Limitationen stehen jedoch eine sorgfältige, literaturgestützte Auswahl und Kontrolle konkurrierender Einflussfaktoren (sog. internale und externale Bedingungen der pädagogischen Betätigung) ebenso entgegen wie die Verwendung spezieller Effektstärkenmaße, die eine Überschätzung der praktischen Bedeutsamkeit ermittelter Fachbereichsunterschiede in kleinen Stichproben verhindern.

Die auf dieser Basis berichteten Ergebnisse lassen interessanterweise zunächst eine Gemeinsamkeit der Unterrichtsgestaltung erkennen: Klassenübergreifend berichten Schüler/innen an Beruflichen Oberschulen von einer hohen Einsatzhäufigkeit der Sozialformen Lehrvortrag und fragend-entwickelndes Lehrgespräch. Dieser Befund korrespondiert mit beobachtungs- und befragungsgestützten Unterrichtsbeschreibungen an dualen Berufsschulen, die dort ebenfalls eine weite Verbreitung frontaler Methoden feststellen (zsf. Götzl, Jahn \& Held 2013).

Die zentrale Frage nach dem relativen Stellenwert fachbereichsabhängiger gegenüber person- und kontextbedingten Variationen (weiterer) unterrichtlicher Praktiken und Überzeugungen ist indes nicht eindeutig zu beantworten. Gegen ein Primat person- und kontextspezifischer Einflüsse (also internaler und externaler Bedingungen) spricht, dass nur wenige der erfassten Variablen in systematischer bivariater Beziehung zu den Kriteriumsvariablen stehen. Bei simultaner Schätzung ihrer Effekte und Einbezug der Fachzugehörigkeit einer Lehrkraft ergeben sich kaum noch eigenständige Erklärungsleistungen. Festzuhalten bleiben aber zwei jeweils inhaltlich kohärente Befunde: Erstens ist ein gelingendes Klassenmanagement systematisch sowohl 
an lehrerseitige Präferenzen für diskursive und anwendungsorientierte Lehrstrategien als auch an schülerseitige Wahrnehmungen einer anwendungsnahen Auseinandersetzung mit fachlichen Lerninhalten gekoppelt. Dieser Befund unterstreicht die Bedeutung einer störungsarmen Lernumgebung für die intensive - und im Falle Beruflicher Oberschulen arbeitsweltlich orientierte Befassung mit Lerninhalten (Ophart \& Thiel 2008). Zweitens erleben Schüler/innen übereinstimmend mit Erkenntnissen der Selbstwirksamkeitsforschung (s. Kapitel 3) den Unterricht bei hoch selbstwirksamen Lehrkräften als stärker anregend und unterstützend als bei Lehrkräften, die ihre eigenen Bewältigungsfähigkeiten beruflicher Anforderungen eher ungünstig einschätzen.

Eine Dominanz des Facheinflusses zeichnet sich wiederum in einigen, nicht aber in allen betrachteten Dimensionen unterrichtlicher Überzeugungen und Praktiken ab. Ein schlüssiges Bild ergibt sich erneut durch eine integrative Betrachtung von Lehrer- und Schüleraussagen, die zugleich potentielle Verzerrungen von Selbst- bzw. Fremdeinschätzungen im Sinne einer gegenseitigen Bestätigung abschwächt: Die befragten Lehrkräfte in wirtschaftswissenschaftlichen sowie mathematisch-naturwissenschaftlichen Fächern messen der transmissiven Strategie Anleiten und Einüben einen höheren Stellenwert bei als den eher konstruktivitischen Strategien Diskurs und Anwendung sowie Entdecken und Differenzieren. Schüler/innen erleben im zugehörigen Fachunterricht einen vergleichsweise geringen Anwendungsbezug, vor allem im Kontrast zu erziehungs- und sozialwissenschaftlichen Fächern. Von ESLehrkräften weichen W-Lehrkräfte zudem überzufällig negativ hinsichtlich einer aus Schülersicht anregenden und unterstützenden Unterrichtsgestaltung $\mathrm{ab}$, während MN-Lehrkräfte signifikant seltener den methodischen Zugang über authentische Problemstellungen der beruflichen Praxis wählen, die kollaborativ zu lösen sind (problemzentrierte Gruppenarbeit).

Bilanzierend betrachtet scheinen in diesen Befunden zumindest erste Konturen fachkulturell geteilter Wahrnehmungs-, Denk- und Handlungsmuster von Lehrkräften an Beruflichen Oberschulen auf, die es in vertiefenden Studien weiter auszudifferenzieren und zu konkretisieren gilt. Obwohl Schülerurteile zum Fachunterricht meist hohe Validität besitzen (Wagner et al. 2013), sind für diese Zwecke ergänzende Unterrichtsbeurteilungen durch trainierte Beobachter sicherlich wertvoll. Zur Aufdeckung fachspezifischer Choreografien des Unterrichtens und zugehöriger Begründungsmuster der Lehrkräfte sind zudem videogestützte Auswertungen in Kombination mit offenen Befragungen wie etwa dem stimulated recall gewinnbringend. Dabei lassen sich auch weitere Kontrollvariablen wie etwa Unterrichtsphasen oder das lehrerindividuelle Niveau fachspezifischen Wissens berücksichtigen. 


\section{Literatur}

Achtenhagen, F. \& Pätzold, G. (2010). Lehr-Lernforschung und Mikrodidaktik. In R. Nickolaus, G. Pätzold \& H. Reinisch, H. (Hrsg.), Handbuch Berufs-und Wirtschaftspädagogik (S. 137-160). Bad Heilbrunn: Klinkhardt UTB.

Buehl, M. M. \& Beck, J. S. (2015). The relationship between teachers' beliefs and teachers' practices. In H. Fives \& M. G. Gill (Eds.), International Handbook of Research on Teachers' Beliefs (pp. 66-84). New York, London: Routledge.

Clement, U. (2006). Curricula für die berufliche Bildung - Fächersystematik oder Situationsorientierung? In R. Arnold \& A. Lipsmeier (Hrsg.) Handbuch der Berufsbildung (S. 260-268). Wiesbaden: VS Verlag für Sozialwissenschaften.

Cohen, J. (1992). Quantitative Methods in Psychology. Psychological Bulletin 112(2), 155-159.

Cross Francis, D., Rapacki, L., \& Eker, A. (2015). The Individual, the context, and practice. A Review of the Research on Teachers' Beliefs Related to Mathematics. In H. Fives \& M. G. Gill (Eds.), International Handbook of Research on Teachers' Beliefs (pp. 336-352). New York, London: Routledge.

Götzl, M., Jahn, R.W. \& Held, G. (2013). Bleibt alles anders?! Sozialformen, Unterrichtsphasen und echte Lernzeit im kaufmännischen Unterricht.bwp@-Berufsund Wirtschaftspädagogik online, 24, 1-22.

Grühn, S. (2000). Unterricht und schulisches Lernen: Schüler als Quellen der Unterrichtsbeschreibung. Münster: Waxmann.

Hericks, U. \& Körber, A. (2007). Methodologische Perspektiven quantitativer und rekonstruktiver Fachkulturforschung in der Schule. In J. Lüders (Hrsg.), Fachkulturforschung in der Schule (S. 31-48). Opladen: Verlag Barbara Budrich.

Huber, L. (1991). Sozialisation in der Hochschule. In K. Hurrelmann \& D. Ulich (Hrsg.), Neues Handbuch der Sozialisationsforschung (S. 417-441). Weinheim: Beltz.

ISB (Staatsinstitut für Schulqualität und Bildungsforschung München) (o.J.). Deutsch an der Beruflichen Oberschule. Online: https://www.isb.bayern.de/beruflicheoberschule/faecher-nach-ausbildungsrichtungen/allgemeinbildende-faecher/ deutsch/ (09.09.2016)

Klassen, R. M. \& Tze, V. M. C. (2014). Teachers' self-efficacy, personality, and teaching effectiveness: A meta-analysis. Educational Research Review 12, 59-76.

König, K. (2014). Die Berufliche Oberschule in Bayern. Geschichte, Organisation, didaktische Konzeption. Bildung und Erziehung, 67(1). 29-68.

Kuntze, S. (2012). Pedagogical content beliefs: global, content domain-related and situation-specific components. Educational Studies in Mathematics, 79(2), 273292.

Lim, C.S., Tang K.N., \& Kor, K.K. (2012). Drill and Practice in Learning (and Beyond). In N. Seel (ed.), Encyclopedia of the Sciences of Learning (pp. 10401042). Wiesbaden: Springer.

Lüders, J. (2007). Einleitung: Fachkulturforschung in der Schule. In J. Lüders (Hrsg.), Fachkulturforschung in der Schule (S. 7-14). Opladen: Barbara Budrich.

Mansour, N. (2009). Science Teachers' Beliefs and Practices: Issues, Implications and Research Agenda. International Journal of Environmental \& Science Education, 4(1), 25-48. 
Müller-Roselius, K. (2007). Habitus und Fachkultur. In J. Lüders (Hrsg.), Fachkulturforschung in der Schule (S.15-30). Opladen: Verlag Barbara Budrich.

Nikolai, R. \& Ebner C. (2011). Wege zwischen beruflicher und hochschulischer Bildung in deutschsprachigen Ländern. Wirtschaftsdienst, 91(1), 42-47.

Ophardt, D. \& Tiehl, F. (2008). Klassenmanagement als Basisdimension der Unterrichtsqualität. In M. K. W. Schwer (Hrsg.), Lehrer-Schüler-Interaktion. Inhaltsfelder, Forschungsperspektiven und methodische Zugänge (S. 259-282). Wiesbaden: VS Verlag für Sozialwissenschaften.

Pahl, J.-P. (2014). Berufsbildende Schule. Bestandsaufnahmen und Perspektiven (2. erweiterte und überarbeitete Aufl.). Bielefeld: Bertelsmann

Reetz, L., \& Seyd, W. (2006). Curriculare Strukturen beruflicher Bildung. In R. Arnold \& A. H. Lipsmeier (Hrsg.), Handbuch der Berufsbildung (S. 227-259). Wiesbaden: VS Verlag für Sozialwissenschaften.

Reusser, K., Pauli, C. \& Elmer, A. (2011). Berufsbezogene Überzeugungen von Lehrerinnen und Lehrern. In E. Terhart, H. Bennewitz \& M. H. Rothland (Hrsg.), Handbuch der Forschung zum Lehrerberuf (S.478-495). Münster: Waxmann.

Seeber, S. \& Squarra, D. (2003). Lehren und lernen in beruflichen Schulen. Schülerurteile zur Unterrichtsqualität. Frankfurt am Main: Peter Lang.

Seidel, T. (2011). Lehrerhandeln im Unterricht. In E. Terhart, H. Bennewitz \& M. Rothland (Hrsg.), Handbuch der Forschung zum Lehrerberuf (S. 605-629). Münster: Waxmann.

Seifried, J. (2009). Unterricht aus der Sicht von Handelslehrern. Frankfurt: Peter Lang.

Sembill, D., Wuttke, E., Seifried, J. Egloffstein, M. \& Rausch, A. (2007). Selbstorganisiertes Lernen in der beruflichen Bildung - Abgrenzungen, Befunde und Konsequenzen. Berufs- und Wirtschaftspädagogik online, 13.

Terhart, E. (2013). Widerstand von Lehrkräften in Schulreformprozessen: Zwischen Kooperation und Obstruktion. In N. McElvany \& H. G. Holtappels (Hrsg.), Empirische Bildungsforschung: Theorien, Methoden, Befunde und Perspektiven (S. 75-92). Münster: Waxmann.

Wagner, W., Göllner, R., Helmke, A., Trautwein, U., \& Lüdtke, O. (2013). Construct validity of student perceptions of instructional quality is high, but not perfect: Dimensionality and generalizability of domain-independent assessments. Learning and Instruction, 28, 1-11.

Willems, Katharina (2007). Doing gender while doing discipline? Zur Macht der Illusion in den Unterrichtsfächern Physik und Deutsch. In J. Lüders, (Hrsg.), Fachkulturforschung in der Schule, (S. 151-167). Opladen: Barbara Budrich.

Willems, H. (2008). Figurationen, Felder, Habitus und Kapitaltypen. In H. Willems (Hrsg.), Lehr(er)buch Soziologie. Für die pädagogischen und soziologischen Studiengänge (Bd. 1, S. 67-88). Wiesbaden: VS Verlag für Sozialwissenschaften.

Winter, H.W. (2016). Entdeckendes Lernen im Mathematikunterricht. Einblicke in die Ideengeschichte und ihre Bedeutung für die Pädagogik (3. aktualisierte Aufl.). Wiesbaden: Springer.

Woolfolk Hoy, A., Hoy, W. K. \& Davis H. A. (2009). Teachers' Self-Efficacy Beliefs. In K. R. Wentzel \& A. Wigfield (Eds.), Handbook of Motivation at School (pp. 627-653). New York, London: Routledge. 
Wuttke, E. (2012). Silence is Silver, Talk is Gold? Analysis of Classroom Talk in A Learner Centred Setting. In E. Hjörne, G. van der Aalsvoort \& G. de Abreu (Eds.), Learning, Social Interaction and Diversity - Exploring Identities in School Practices (pp. 103-117). Rotterdam, Bosten, Taipeh: SensePublishers. 



\title{
Die Öffnung des Hochschulzugangs für beruflich Gebildete: Förderung studienrelevanter Schlüsselkompetenzen in der Studieneingangsphase am Beispiel der Universität Kassel
}

\author{
Christian Schmidt
}

\section{Einleitung}

Die mit der Ausweitung des Hochschulzugangs verbundene größere Heterogenität der Studierenden in Bezug auf die Studierfähigkeit stellt Hochschulen vor allem in der Studieneingangsphase vor große Herausforderungen. Die Förderrichtlinien des Qualitätspakts Lehre sehen aktuell eine Förderung von Maßnahmen zur Entwicklung innovativer Studienmodelle zur Ausgestaltung der Studieneingangsphase in Hinblick auf eine heterogener gewordenen Studierendenschaft vor (vgl. Bundesministerium für Bildung und Forschung 2014, 2) und so werden aktuell viele Programme mit dieser Zielsetzung gefördert. Beispielhaft sei hier die Studie von Bosse und Trautwein (,Hamburger Modell - Studierfähigkeit“) zu individuellen und institutionellen Herausforderungen der Studieneingangsphase genannt (vgl. Bosse \& Trautwein 2014).

Die zentrale Fragestellung des Beitrags lautet vor diesem Hintergrund, wie die Studieneingangsphase Heterogenität als Problemstellung strukturell aufgreifen kann und inwieweit Lehrangebote Kompetenzentwicklung und reflektion im Kontext des wissenschaftlichen Arbeitens mit den fachlichen Inhalten verbinden können. Zunächst werden hierzu Ansätze aus dem Fachdiskurs dargestellt und das Projekt ProBeg L4 als ein auf Binnendifferenzierung und Kompetenzrastern basierender Ansatz in der Lehramtsausbildung für Berufsbildende Schulen an der Universität Kasse ausgeführt. ${ }^{1}$ Der Innovationsgehalt und Beitrag zu Theorie und Praxis werden abschließend diskutiert.

1 „Professionsbezogene Begleitung der L4-Studierenden unter Berücksichtigung heterogener Eingangsvoraussetzungen (ProBeg L4)“ ist ein Teilprojekt von PRONET (Professionalisierung durch Vernetzung) der Universität Kassel und wird im Rahmen der Qualitätsoffensive Lehrerbildung des BMBF gefördert. 


\section{Die Ausweitung des Hochschulzugangs für beruflich Qualifizierte}

Die Durchlässigkeit des Bildungssystems, die Verschränkung beruflicher und höherer Allgemeinbildung und die Anerkennung beruflicher Kompetenzen in Bezug auf den Hochschulzugang sind Themenstellungen, die im Kontext des demographischen Wandels sowie des Europäischen und Deutschen Qualifikationsrahmens und des prognostizierten Fachkräftemangels wieder verstärkt in der wissenschaftlichen Fachdiskussion stehen (vgl. Münk, Walter \& Schmidt 2013). Neben der traditionellen Argumentationslinie, die seit den 1960er Jahren aus Gründen der Bildungsgerechtigkeit und der bildungstheoretischen Gleichstellung beruflicher und höherer Allgemeinbildung der Durchlässigkeit von beruflicher Bildung und Hochschulbildung das Wort redet, wird aktuell auch aus der Perspektive eines drohenden Fachkräftemangels dafür plädiert, Karrieremodelle von der beruflichen Bildung in die Hochschule zu fördern (vgl. Dobischat et al. 2016, 60 ff.).

Die Möglichkeit des Erwerbs eines Hochschulzugangs nach der Berufsausbildung und eventuell folgender Berufstätigkeit z. B. an berufsbildenden Schulen oder aber auch an Abendgymnasien besteht schon lange. Berufliche Gymnasien sowie Berufs- und Fachoberschulen stellen hierfür geschaffene Institutionen dar. Außerdem existieren Möglichkeiten des Hochschulzugangs für beruflich Qualifizierte aufgrund von beruflicher Qualifikation und/ oder Berufserfahrungen (vgl. BIBB 2016, 416). Hier ist zum einen der Erwerb der allgemeinen Hochschulzugangsberechtigung mit der Fortbildungsprüfung (z. B. die Meister- oder Technikerprüfung) zu nennen (vgl. Schütz 2014, 201), zum anderen existieren in bestimmten Bundesländern universitäre Hochschulzugangsprüfungen für beruflich Qualifizierte ohne die genannten Hochschulzugänge, welche den Zugang zu bestimmten Studienbereichen ermöglichen (vgl. für Hessen: BQuHSchulZVO 2015).

Durch eine solche Öffnung der Hochschulen bekommt die alte Debatte um die Gleichwertigkeit beruflicher Bildung und höherer Allgemeinbildung eine neue Dimension, da nun neben der formalen oder bildungstheoretischen Gleichwertigkeit sehr viel stärker die Frage im Raum steht, inwieweit berufliche Bildungswege tatsächlich für ein Hochschulstudium qualifizieren. Vielfach wird diskutiert, dass mit Ausweitung des Hochschulzugangs die Studierenden heterogener bezüglich der studiumsrelevanten Kompetenzen seien und das Thema „Studierfähigkeit“ an Relevanz gewönne (vgl. Hanft 2015, 15 ff.; 20 ff.).

Diese Fragestellungen treffen auf einen hochschulpolitischen Diskurs, der ein unzureichendes Leistungsniveau der Studierenden konstatiert und häufig eine kritische Haltung zur Studienstrukturreform einnimmt (vgl. Bosse \& Trautwein 2014, 42). Eine Diskrepanz zwischen den Lebenslagen und 
Qualifikationsprofilen der Studierenden und den Angebotsstrukturen sowie Studienanforderungen in der Studieneingangsphase wird vermutet (vgl. Brahm, Jenert \& Wagner 2014, 43). Die genannten Fragestellungen sind aber ebenso als Teil des Diskurses um Lebenslanges Lernen zu verorten, der die Notwendigkeit lebenslanger Qualifizierung zur Aufrechterhaltung der „Employability“ unterstreicht. Dies führt dazu, dass Übergänge aus der beruflichen Erstausbildung in Hochschulbildung ebenso an Bedeutung gewinnen, wie die Verknüpfung von Hochschulbildung und beruflicher Weiterbildung. Euler spricht von „Überlappungen zwischen dem unteren Rand der Hochschulbildung und dem oberen Rand der beruflichen Bildung" (Euler 2017, 42), verweist auf das Wachstum hybrider Bildungsgänge wie duale Studiengänge und konstatiert, dass die Durchlässigkeit für beruflich Qualifizierte nicht mehr nur eine Forderung der Berufsbildung, sondern eine bildungspolitische Vorgabe sei (vgl. ebd.).

Bei den an Universitäten verankerten Studiengängen könnten vor allem die Lehramtsstudiengänge für Berufsbildende Schulen von einer größeren Heterogenität der Studierfähigkeit aufgrund der Öffnung der Hochschule betroffen sein. So stellen Studiengänge des Lehramts für Berufsbildende Schulen Angebote dar, die häufig auch von jungen Menschen ohne akademischen familiären Hintergrund gewählt werden (vgl. Sonntag 2016, 62, 101). Außerdem liegt es gerade für Studienanfänger, die über den beruflichen Bildungsweg einen Hochschulzugang erworben haben, nahe, das Lehramt an berufsbildenden Schulen in ihre akademischen Bildungsaspirationen einzubeziehen, da sie den Lernort berufsbildende Schule ja bereits kennen.

Die Lehramtsausbildung für berufsbildende Schulen steht also ganz besonders vor der Herausforderung, auf die Öffnung des Hochschulzugangs und die größere Heterogenität bezüglich der Studierfähigkeit in der Studieneingangsphase zu reagieren. Gerade in den technisch-gewerblichen Fachrichtungen, die von einem großen LehrerInnenmangel betroffen sind, erscheint es überlebensnotwendig, diese Frage zu adressieren.

\section{Heterogenität in der Studieneingangsphase und didaktische Konzeptionen}

\section{Empirische Befunde zum Studienerfolg}

Empirische Forschungsbefunde zum Studienerfolg beruflich gebildeter Studierender zeichnen ein uneinheitliches Bild. Insgesamt erscheint die empirische Forschungslage keine grundlegenden Unterschiede bezüglich des Studienerfolgs von beruflich Qualifizierten und traditionellen Studierendengruppen festzustellen (vgl. zusammenfassend Grendel, Lübbe \& Haußmann 2014, 
44). Teilweise werden allerdings größere Übergangsprobleme in der Studieneingangsphase von beruflich Qualifizierten konstatiert (vgl. ebd.). Grendel, Lübbe und Haußmann konnten zeigen, dass mit zunehmender Dauer der Berufserfahrung beruflich Gebildeter der Studienerfolg zunimmt, andererseits die fachliche Nähe der vor dem Hochschulstudium ausgeübten Berufstätigkeit keinen Einfluss auf den Studienerfolg nimmt (vgl. Grendel, Lübbe \& Haußmann 2014, 58).

Eine Studie zum Zusammenhang zwischen der Art des Hochschulzugangs und dem Studienerfolg im Studiengang Wirtschaftspädagogik an der Universität Kassel konnte feststellen, dass mit der allgemeinen Hochschulreife eine größere Erfolgsquote in den Modulprüfungen zu verzeichnen war, als mit einer Fachhochschulreife (vgl. Sonntag 2016, 169). Insgesamt hatten hier vor allem Studierende mit Fachhochschulzugang und ohne Ausbildung deutlich geringere Erfolgsquoten in den Prüfungen. Bei den Abschlussnoten des Gesamtstudiengangs waren Studierende ohne allgemeine Hochschulreife signifikant seltener in der notenbesten Gruppe (vgl. Sonntag 2016, 227). Auch bezüglich der Studiendauer und der Anzahl von Fehlversuchen schnitten Studierende mit allgemeiner Hochschulreife besser ab (vgl. Sonntag 2016, 198).

\section{Didaktische Konzeptionen im Fachdiskurs}

Die gestiegene Bedeutung der universitären Studieneingangsphase schlägt sich in vielfältigen Fachveröffentlichungen zu dem Thema nieder. Hier wird diese verstärkt als Bereich der Reflexion von studienerfolgsrelevanten Kompetenzen und der Orientierung angesehen. So heben Bochert und Bestmann die Implementierung von Gelegenheiten der Kompetenzreflexion in der Studieneingangsphase als gerade für beruflich gebildete Studierende wichtig hervor und entwickeln entsprechende Seminareinheiten (vgl. Bochert \& Bestmann 2015, 213 ff.). Zur Orientierung, Organisation und methodologischen Reflexion werden E-Learning-Angebote (vgl. Krebs 2013) und EPortfolios (vgl. van den Berg \& Tan 2013; Merkt 2007) diskutiert. Brückenkurskonzepte stellen auf die Notwendigkeit einer angemessenen Propädeutik ab (vgl. Poppinga, Wetzel \& Marlen 2015). Aber auch die Notwendigkeit einer „sinnvollen Selbstprüfung bezüglich Studienfachwahl und Studierfähigkeit" (Schallert; Budka; Payrhuber 2008, 278) wird unterstrichen. Elaborierte didaktische Konzepte zielen auf die Reflexion der Lernstile seitens der Studierenden (vgl. Hultberg et. al. 2008; Kjellgren et. al. 2008). KrügerBasener und Rabe setzen in Mathematik-Vorkursen gezielt Binnendifferenzierung als didaktisches Mittel ein, um Leistungsunterschiede konzeptionell zu berücksichtigen (vgl. Krüger-Basener \& Rabe 2014, 313 ff.). Ziel fast aller Ansätze ist die Verringerung von Studienabbruchsquoten und ein besserer Übergang in die akademisch-wissenschaftliche Lehr-Lernkultur (vgl. van den Berg \& Tan 2013, 219 f.; Poppinga, Wetzel \& Marlen 2015). 
Vor allem an Fachhochschulen existieren bereits implementierte Konzepte zur Studieneingangsphase, die häufig auf Brückenkurse in als besonders schwierig eingeschätzten Fächern oder Inhalten (vgl. Krüger-Basener \& Rabe 2014) oder dem Fachstudium vorgelagerte Orientierungsstudiengänge abstellen (vgl. Raue \& Schröder 2014, 185 ff.).

Insgesamt fällt auf, dass die Ansätze in der Regel die bestehenden LehrLernformen der Universität durch Angebote ergänzen, die Kompetenzreflexion initiieren (gesonderte Seminare, zusätzlich zu führende E-Portfolios, zusätzliche Web-based Trainings) (vgl. Bochert \& Bestmann 2015, 213ff; Krebs 2013, 244; van den Berg \& Tan 2013, 224) oder als zusätzliche Angebote Defizite abbauen sollen (vgl. Poppinga, Wetzel \& Marlen 2015). Verkürzt könnte man zusammenfassen, es gehe um Reflexion on the top und defizitorientierte Anpassung an einen einheitlichen Erwartungshorizont anstatt struktureller Anpassung an Heterogenität.

Auch fällt auf, dass ein Bezug zu hochschuldidaktischen Konzepten, abgesehen von der Betonung der Förderung von Reflexion als Voraussetzung für vertieftes Lernen und die Entwicklung mentaler Modelle (vgl. Merkt 2007, 291), häufig ausbleibt. Ein solcher theoretischer hochschuldidaktischer Ansatzpunkt stellt das Constructive Alignment dar, welches die quantitative Ausweitung und erhöhte Heterogenität der Studierendenschaft im Zuge der Bildungsexpansion zum Ausgangspunkt nimmt und auf daraus resultierend veränderte Motivationsstrukturen und Wissensbestände sowie Lernstile eingeht (vgl. Biggs \& Tang 2007). Stark verkürzt geht der Ansatz davon aus, dass die größere Heterogenität der Studierendenschaft heute ein verändertes Studienverhalten bedinge: Studierenden, die sich in Lernstil und Lernmotivation an den konkreten Prüfungsvorgaben ausrichteten und ein eher instrumentelles Verhältnis zum Hochschulstudium hätten, stellten die Mehrheit gegenüber intrinsisch an den Inhalten akademischer Lehrveranstaltungen orientierten Studierenden (vgl. Biggs \& Tang 2007, 8). Diese Motivation führe zu oberflächlichem Lernen. Der Ansatz zielt darauf ab, einen engen Bezug zwischen erwarteten Lernzielen, kompetenzorientierter Prüfung und didaktischmethodischer Gestaltung der Lehrveranstaltung herzustellen, um auch ursprünglich oberflächlich Lernende zu einer vertieften akademischen Auseinandersetzung mit universitären Angeboten zu bringen. Zentral ist in diesem Kontext eine klare Kommunikation bezüglich der Learning Outcomes (vgl. Biggs \& Tang 2007, 21 ff.) Diesem Ansatz entsprechend sollten Lehrveranstaltungen in der Studieneingangsphase die Reflexionsanlässe bezüglich der eigenen studienrelevanten Kompetenzen integrieren und klar darlegen, welches Niveau in den entsprechenden Kompetenzen erreicht werden sollte, um die Grundlagenveranstaltungen erfolgreich zu absolvieren. Die Reflexion sollte in einem Zusammenhang mit den Lernzielen der grundlegenden Lehrveranstaltungen stehen. Außerdem kann ein erfolgversprechender Ansatz darin liegen, der Heterogenität durch binnendifferenzierte Lehrformen zu 
begegnen, die Reflexion und Kompetenzerwerb auf unterschiedlichen Niveaustufen ermöglichen. Im folgenden Kapitel wird ein solcher Ansatz an der Universität Kassel vorgestellt.

\section{Struktur und Intention des Projekts „Professionsbezogene Begleitung der L4-Studierenden unter Berücksichtigung heterogener Eingangsvoraussetzungen (ProBeg L4)“}

Traditionell stellt der Universitätsstandort Kassel ein Studienangebot, welches viele beruflich gebildete Studierende aufnimmt. Dies ist zum einen begünstigt durch die Tatsache, dass in Hessen mit der Fachhochschulreife ein Hochschulstudium begonnen werden kann, zum anderen durch das Gründungsprogramm als Gesamthochschule. So verfügen $25 \%$ der Studierenden in Kassel über eine Fachhochschulreife (bundesweit: $2 \%$ ) und $53 \%$ der Studierenden haben kein Elternteil mit akademischer Bildung (bundesweit: 37 \%) (vgl. Sonntag 2016, 9 ff.). Im Studiengang Wirtschaftspädagogik stellten die Erstsemester mit Fachhochschulreife oder fachgebundener Hochschulreife im Wintersemester 2014/15 mit 72,8\% deutlich die Mehrheit, während 26,6 \% mit der allgemeinen Hochschulreife das Studium begannen. Studierende, die mit einer Fortbildungsprüfung o. ä. ein Studium aufnehmen, stellten mit $6 \%$ eine Minderheit dar (vgl. Sonntag 2016, 7). Somit kann der genannte Studiengang als typisches Beispiel für eine größere Heterogenität in der Studieneingangsphase gelten. An der Universität Kassel wurden Empfehlungen zum Umgang mit Heterogenität in der Lehre entwickelt, welche die Notwendigkeit einer frühen Diagnostik und Förderung von Schlüsselkompetenzen rund um die Studierfähigkeit sowie zur Binnendifferenzierung in den Lehrangeboten hervorheben (vgl. AG Heterogenität 2009, 7 ff.).

Im Projekt ProBeg L4 wird erprobt, durch eine Verbindung von Selbstassessments und binnendifferenzierten Grundlagenveranstaltungen eine der heterogenen Studierendenschaft angemessene Form der Förderung studienerfolgsrelevanter Schlüsselkompetenzen zu erreichen und auch eine Klärung bezüglich der Anforderungen eines universitären Studiums und somit eine informierte Studienentscheidung frühzeitig herbeizuführen.

Individuelle Selbstassessments der Studierfähigkeit über Kompetenzraster in Verbindung mit Arbeitsaufgaben unterstützten im erprobten Ansatz die Einstufung in unterschiedliche Lernstufen. Die Studierenden evaluieren ihre Kompetenzen anhand eines entsprechenden Kompetenzrasters und wählen den Kompetenzen entsprechende Lernstufen. Das Kompetenzraster organisiert Kompetenzen des Verständnisses wissenschaftlicher Texte, der wissen- 
schaftlichen Ausdrucksfähigkeit und Arbeitsweise und der informieren Studienwahl auf vier Niveaustufen.

Abb. 1.: Kompetenzraster ${ }^{2}$ (Ausschnitt)

\begin{tabular}{|c|c|c|}
\hline $\begin{array}{l}\text { Anforderungen } \\
\text { in der Studien- } \\
\text { eingangsphase }\end{array}$ & Niveau 1 & Niveau 2 \\
\hline \multirow{2}{*}{$\begin{array}{l}\text { Verständnis wissen- } \\
\text { schaftlicher Texte }\end{array}$} & $\begin{array}{l}\text { Ich verstehe wissenschaftliche } \\
\text { Texte und Statistiken und kann } \\
\text { einzelne Aussagen wiedergeben. }\end{array}$ & $\begin{array}{l}\text { Ich verstehe wissenschaftliche } \\
\text { Texte umfänglich und kann wichti- } \\
\text { gere von weniger wichtigen Aussa- } \\
\text { gen unterscheiden. }\end{array}$ \\
\hline & $\begin{array}{l}\text { Ich kann die theoretischen Grund- } \\
\text { lagen des Textes benennen. }\end{array}$ & $\begin{array}{l}\text { Ich kann Argumente gedanklich } \\
\text { vor dem Hintergrund theoretischer } \\
\text { Grundlagen eines Textes einord- } \\
\text { nen. }\end{array}$ \\
\hline \multirow{3}{*}{$\begin{array}{l}\text { (Wissenschafts-) } \\
\text { sprachliche } \\
\text { Ausdrucks } \\
\text { fähigkeit }\end{array}$} & $\begin{array}{l}\text { Ich habe etwas Erfahrung bei der } \\
\text { selbstständigen Produktion von } \\
\text { Texten, bin aber bei Orthografie } \\
\text { und Grammatik sehr unsicher. In } \\
\text { der Anwendung von Wissen- } \\
\text { schaftssprache bin ich unerfahren. }\end{array}$ & $\begin{array}{l}\text { Ich habe Erfahrung bei der selbst- } \\
\text { ständigen Produktion von Texten, } \\
\text { mache aber manchmal noch Fehler } \\
\text { bei Orthografie und Grammatik. } \\
\text { Ich habe bereits erste Erfahrungen } \\
\text { mit Fachvokabular gesammelt. }\end{array}$ \\
\hline & $\begin{array}{l}\text { Ich kann klar und verständlich } \\
\text { formulieren. }\end{array}$ & Ich kann sachbezogen formulieren. \\
\hline & $\begin{array}{l}\text { Ich kann im Text aufgeführte } \\
\text { Fakten und Argumente korrekt } \\
\text { widergeben und sorgfältig be- } \\
\text { schreiben. }\end{array}$ & $\begin{array}{l}\text { Ich kann Aussagen eines Textes in } \\
\text { Beziehung setzen und dies in } \\
\text { nachvollziehbarer Weise schriftlich } \\
\text { formulieren. }\end{array}$ \\
\hline
\end{tabular}

Quelle: Eigene Darstellung

Neben der reflexiven Einordnung in die Lernstufen durch die Studierenden sollen die Assessments ganz unmittelbar auch die Notwendigkeit der Kompetenzentwicklung unterstreichen. Durch das Assessment in mehreren aufeinanderfolgenden Lehrveranstaltungen in der Studieneingangsphase (die ersten zwei Semester) wird die Reflexion einer längeren individuellen Kompetenzentwicklung ermöglicht. Die Dokumentation des langfristigen Fortschritts in den Selbstassessments wird über ein E-Portfolio sichergestellt. Der portfoliogestützte Gebrauch des Rasters vermittelt, dass mit dem Erreichen der Niveaustufen drei und vier ein Mindestniveau bezogen auf die Studierfähigkeit erreicht wird. Die Kompetenzraster werden regelmäßig in den tutoriellen Angeboten und Seminaren reflektiert und der Zusammenhang zwischen erwarteten Kompetenzen und den Modulprüfungen verdeutlicht. Methodisch orientieren sich die Assessments nicht an den verbreiteten retrospektiven

2 Das Kompetenzraster wurde im Projektkontext auf der Grundlage der von Bosse und Trautwein analysierten kritischen Studienanforderungen entwickelt (vgl. Bosse \& Trautwein 2014, 48f.). 
Selbstberichten zu Lernstrategien bzw. Lernstrategiefragebögen, die zwar Selbsteinschätzungen erfassen, diese aber selten in einem empirisch gesicherten Zusammenhang zum tatsächlichen Lernverhalten stehen. Stattdessen wird in Anlehnung an Artlet (vgl. Artlet 2000) das jeweils zu Beginn und zum Ende der Grundlagenveranstaltungen stattfindende Assessment mit Aufgaben zur Textinterpretation verbunden, um den tatsächlichen Strategiegebrauch im Handlungskontext in die Selbsteinschätzung der relevanten Kompetenzen einfließen zu lassen.

\section{Abb. 2.: Assessments und Lernstufen in aufeinander folgenden Lehrveranstaltungen}

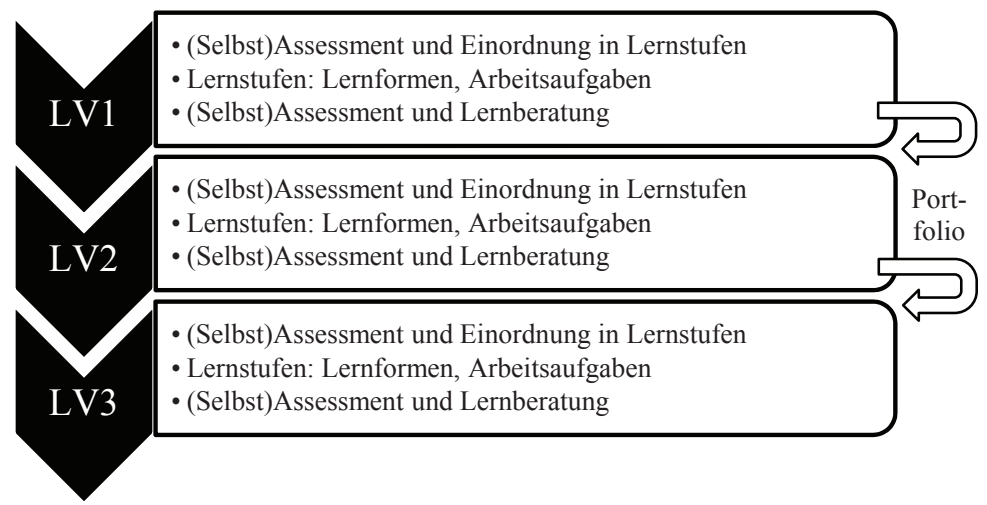

Quelle: Eigene Darstellung

Die Lernstufen selbst unterscheiden sich hinsichtlich des Anteils von Selbsterarbeitungsphasen oder assistierter Arbeitsphasen und der Unterstützung bei Literaturrecherchen. Studierende, die sich in den studienerfolgsrelevanten Kompetenzen auf einer niedrigen Lernstufe einordnen, bekommen eine höhere Unterstützung bei der Literaturrecherche und Vorgaben bei der zu verwendenden Literatur, haben einen moderaten Anteil an Selbsterarbeitungsphasen und häufigere Präsenztermine als Studierende in den höheren Lernstufen. Bis zum Ende der Studieneingangsphase sollen so unterschiedliche Studienvoraussetzungen kompensiert sowie Studienabbrüchen und Verzögerungen im Studienverlauf präventiv entgegengewirkt werden. Lernberatung und tutorielle Begleitung erfolgen auf der Grundlage einer individuellen Reflexion der Kompetenzentwicklung und verweisen auf der Grundlage des sichtbaren Kompetenzstandes auf weiterführende unterstützende Angebote des Service- 
center Lehre. ${ }^{3}$ Hochschuldidaktisch orientiert sich der Ansatz am Constructive Alignment (vgl. Biggs \& Tang 2007), da Lehre, Kompetenzerwartungen und Prüfung bzw. Assessment in enger Abstimmung erfolgen.

\section{Diskussion und Fazit}

Der Ansatz, die Selbsteinschätzung relevanter Schlüsselkompetenzen im Studium mit Portfolioarbeit und binnendifferenzierten Grundlagenveranstaltungen zu verbinden, adressiert zentrale Probleme, die mit der Öffnung der Hochschulen für beruflich Qualifizierte in Verbindung stehen. Er stimmt die Kompetenzentwicklung zur Studierfähigkeit mit den inhaltlichen Anforderungen in der Studieneingangsphase ab und bezieht sich somit auf die eingangs formulierte Fragestellung nach strukturellen Ansätzen zum Umgang mit Heterogenität.

Der in Kapitel 2 formulierten Kritik an manchen didaktische Konzeptionen und Modellen, die lediglich additiv zum inhaltlichen Studium Gelegenheiten der Reflexion anbieten, stellt er eine Perspektive entgegen, welche diese Reflektion durch die Anbindung an Grundlagenmodule verbindlicher gestaltet.

Da Evaluationsergebnisse noch nicht vorliegen, kann der Erfolg des Ansatzes nicht beurteilt werden. Wenn die Annahmen des Constructive Alignments allerdings stimmen und mit zunehmender Heterogenität eine Mehrheit der Studierenden ein eher instrumentelles Verhältnis zum Hochschulstudium entwickelt, sollten Lehrangebote in der Studieneingangsphase den Zusammenhang zwischen dem Erwerb propädeutischer Schlüsselkompetenzen und Studienerfolg verdeutlichen. In Fokus steht tatsächlich die Verdeutlichung eines mit universitärem Lehren und Lernen verbundenen Anspruchs an selbstständigem Kompetenzerwerb.

Theoretisch verweist der Ansatz auf die grundlegende Fragestellung nach der Anpassung des Systems (hier die Hochschule) an die zunehmende Heterogenität der Bildungsteilnehmer. Dabei stellt Binnendifferenzierung ein hochschuldidaktisch wenig genutztes und beforschtes Themenfeld dar. Allerdings werden zurzeit viele Modelle propädeutischer Maßnahmen, gerade auch in den gewerblich-technischen Lehramtsausbildungen erprobt, die zumindest im Ansatz Anknüpfungspunkte für je nach Kompetenzstand unterschiedliche Lerngruppen bieten. Für die Zukunft ist zu erwarten, dass die Evaluation und wissenschaftliche Begleitung der QSL-geförderten Projekte

3 Das Servicecenter Lehre stellt eine Serviceeinrichtung der Universität Kassel dar, die hochschulweit und unabhängig von konkreten fachlichen Lehrangeboten Seminare zur Verbesserung der Studierfähigkeit anbietet. 
zur Studieneingangsphase Erkenntnisse zur Wirksamkeit unterschiedlicher Ansätze liefern. Dies ist ein wichtiger Baustein für eine evidenzbasierte Bewertung zum Übergang aus beruflicher Bildung in Hochschulbildung und die alte Frage zur Gleichwertigkeit beruflicher und höherer Allgemeinbildung. Zentral erscheint, die Debatte von ihrem bildungspolitischen und bildungstheoretischen Ausgangspunkt aus in Richtung der Frage weiterzuentwickeln, inwiefern berufliche Kompetenzen und ganz allgemein Kompetenzen, die mit beruflicher Bildung und beruflicher Aufstiegsfortbildung erworben werden, $\mathrm{zu}$ einem erfolgreichen Studieneinstieg beitragen.

Die Frage nach anderen Strukturen und pädagogischen Interventionen in der Studieneingangsphase steht letztendlich vor einer problematischen Zielklärung: Zum einen kann es nicht darum gehen, in Zeiten einer großen Ausweitung der Studierendenquoten das Niveau universitärer Ausbildung zu senken. Auch ist die deutsche Universität personell und hochschuldidaktisch nicht in der Lage, eine Funktion zu erfüllen wie sie manche CollegeStrukturen im angelsächsischen Bereich wahrnehmen, die quasi eine Brückenfunktion zwischen den Lehr-Lern-Arrangements der Sekundarstufe II und einer auf selbstständigem Lernen ausgerichteten universitären LehrLernkultur darstellen. Andererseits müssen Universitäten in der Studieneingangsphase institutionell und hochschuldidaktisch auf die größere Heterogenität der Studienanfänger eingehen und Orientierungsprozesse sowie die Übernahme des universitären Lernmodus unterstützen. Die Frage nach der universitären Sozialisation stellt sich ebenfalls neu, wenn die Bildungswege der StudienanfängerInnen heterogener werden. Letztendlich verlagern sich die Aufgaben der Herstellung von Studierfähigkeit und der individuellen Klärung der Frage, ob die universitäre Form des Bildungserwerbs die individuell richtige ist, in die Studieneingangsphase.

\section{Literatur}

AG Heterogenität (2009). Empfehlungen zum Umgang mit der Heterogenität in den Lernvoraussetzungen der Studierenden. o. Ort.

Artelt, Cordula (2000). Wie prädiktiv sind retrospektive Selbstberichte über den Gebrauch von Lernstrategien für strategisches Lernen? Zeitschrift für Pädagogische Psychologie, 1-10.

Berg, I. \& Tan, W.-H. (2013). Das wissenschaftlich-akademische E-Portfolio in der Studieneingangsphase. In C. Bremer \& D. Krömker (Hrsg.), E-Learning zwischen Vision und Alltag: zum Stand der Dinge (S. 219-229). Münster: Waxmann.

Biggs, J. B. \& Tang, C. (2007). Teaching for quality learning at university. What the student does. 3. Ed., reprinted. Maidenhead: McGraw-Hill (McGraw-Hill education). 
Bochert, N. \& Bestmann, S. (2015). Kompetenzreflexion - Impulse hochschulischer Praxis zur Förderung kompetenzorientierter und partizipativer Lehr-Lernsettings in der Studieneingangsphase berufsbegleitender Studiengänge. In B. Klages, M. Bonillo, S. Reinders \& A. Bohmeyer (Hrsg.), Gestaltungsraum Hochschullehre. Potentiale nicht-traditioneller Studierender nutzen (S. 211-225). Opladen; Berlin; Toronto: Budrich UniPress.

Bosse, E. \& Trautwein, C. (2014). Individuelle und institutionelle Herausforderungen der Studieneingangsphase. Zeitschrift für Hochschulentwicklung, 9 (5), 41-62.

Brahm, T., Jenert, T., Wagner, D. (2014). Nicht für alle gleich - Subjektive Wahrnehmung des Übergangs Schule - Hochschule. Zeitschrift für Hochschulentwicklung, 9 (5), 63-82.

Bundesinstitut für Berufliche Bildung (Hrsg.) (2016). Datenreport zum Berufsbildungsbericht. Bonn. Online: https:/www.bibb.de/dokumente/pdf/bibb_datenreport_2016.pdf (30.11.2016).

Bundesministerium für Bildung und Forschung (2014). Verbindliche Regelungen zur Erstellung von Fortsetzungsanträgen im Bund-Länder-Programm für bessere Studienbedingungen und mehr Qualität in der Lehre. Online: http://www.qualitaetspakt-lehre.de/_media/Foerderaufruf_Periode_2.pdf (12.12.2016).

BQuHSchulZVO (2015). Verordnung über den Zugang beruflich Qualifizierter zu den Hochschulen im Lande Hessen vom 16. Dezember 2015. Gesetz- und Verordnungsblatt für das Land Hessen - 30. Dezember 2015, 655-659.

Dobischat, R., Schäfer, A., Schmidt, C, Wahle, M. \& Walter, M. (2016). Berufslaufbahnkonzepte: Instrumente zur Weiterentwicklung des Berufsbildungssystems? In J. Seifried, S. Seeber \& B. Ziegler (Hrsg.), Jahrbuch der berufs- und wirtschaftspädagogischen Forschung 2016 (S. 59-72). Opladen: Budrich.

Euler, D. (2017). Gleichartig aber nicht gleichwertig? - Überlegungen zu einer Neubestimmung des Verhältnisses zwischen Berufs- und Hochschulbildung. In D. Münk \& M. Walter (Hrsg.), Lebenslanges Lernen im sozialstrukturellen Wandel. Ambivalenzen der Gestaltung von Berufsbiografien in der Moderne (S. 39-62). Wiesbaden: Springer.

Grendel, T., Lübbe, H. \& Haußmann, I. (2014). Effekte der Dauer und der Qualität berufspraktischer Vorerfahrungen auf den Studienerfolg beruflich Qualifizierter. Beiträge zur Hochschulforschung, 36 (4), 40-63.

Hanft, A. (2015). Heterogene Studierende - homogene Studienstrukturen. In A. Hanft, O. Zawacki-Richter \& W. Gierke (Hrsg.): Herausforderung Heterogenität beim Übergang in die Hochschule (S. 13-28). Waxmann: Münster.

Hultberg, J., Plos, K., Hendry, G. \& Kjellgren, K. (2008). Scaffolding students' transition to higher education: parallel itroductory courses for students and teachers. Journal of Further and Higher Education, 32, 47-57.

Kjellgren, K., Hendry, G., Hultberg, J., Plos, K., Rydmark, M., Tobin, G. \& Saljö, R. (2006). Learning to Learn an learning to teach - Introduction tot studies to higher education. In: Medical Teacher (30), e239-e245. Online: http://www.tandfonline. com/doi/pdf/10.1080/01421590802258896?needAccess=true (06.04.2017).

Krebs, J. (2013). E-Learning in der Eingangsphase des Philosophiestudiums. In C. Bremer \& D. Krömker (Hrsg.), E-Learning zwischen Vision und Alltag: zum Stand der Dinge (S. 241-245). Münster: Waxmann. 
Krüger-Basener, M. \& Rabe, D. (2014). Mathe0 - der Einführungskurs für alle Erstsemester einer technischen Lehreinheit. In I. Bausch, R. Biehler, R. Bruder, P. R. Fischer, R. Hochmuth, W. Koepf et al. (Hrsg.), Mathematische Vor- und Brückenkurse. Konzepte, Probleme und Perspektiven (S. 309-324). Wiesbaden: Springer Spektrum.

Merkt, M. (2007). ePortfolios - der ,,rote Faden“ zur Kompetenzentwicklung in Bachelor-Masterstudiengängen. In M. Merkt, K. Mayrberger, R. Schulmeister, A. Sommer \& I. Berk (Hrsg.), Studieren neu erfinden - Hochschule neu denken (S. 285-295). Münster: Waxmann.

Münk, D. (2017). The Dark Side of lifelong Learning: Unzeitgemäße Überlegungen zu einem zeitgemäßen Problem. In D. Münk \& M. Walter (Hrsg.), Lebenslanges Lernen im sozialstrukturellen Wandel. Ambivalenzen der Gestaltung von Berufsbiografien in der Moderne (S. 3-38). Wiesbaden: Springer.

Münk, D., Walter, M. \& Schmidt, C. (2013). Berufliche Bildung und demografischer Wandel: Herausforderungen und Gestaltungsaufgaben für die moderne Arbeitsgesellschaft im Umbruch. In G. Niedermair (Hrsg.), Facetten berufs- und betriebspädagogischer Forschung. Grundlagen, Herausforderungen und Perspektiven (S. 407-430). Linz: Trauner.

Poppinga, T., Wetzel, K. \& Arnold, M. (2015). Elemente innovativer Brückenkurskonzepte in der wissenschaftlichen Weiterbildung für den Abbau von Abbruchquoten in mathematisch-naturwissenschaftlichen Studiengängen. Hochschule und Weiterbildung, 1, 62-69.

Raue, C. \& Schröder, C. (2014). Das Orientierungsstudium Mintgrün: flankierter Systemübertritt von Schule zu Hochschule. Zeitschrift für Hochschulentwicklung, 9 (5), 179-199.

Schallert, C., Budka, P. \& Payrhuber, A. (2008). Die interaktive Vorlesung. Ein Blended-Learning-Modell für Massenvorlesungen im Rahmen der gemeinsamen Studieneingangsphase der Fakultät für Sozialwissenschaften (eSOWI-STEP). In S. Zauchner, P. Baumgartner, E. Blaschitz, \& A. Weissenbäck (Hrsg.), Offener Bildungsraum Hochschule. Freiheiten und Notwendigkeiten (S. 275-286). Münster: Waxmann.

Schütz, A. (2014). Durchlässigkeit der Bildungssysteme - die Rettung zur Sicherung des Angebots an (qualifizierten) Fachkräften? In M. Brucherseifer, D. Münk \& M. Walter (Hrsg.), Arbeit der Zukunft - Zukunft der Arbeit. Berufliche Bildung, Qualifikation und Fachkräftebedarf im Zeichen des demographischen Wandels (S. 199-212). Bonn: Pahl-Rugenstein.

Sonntag, G. (2016). Studienerfolg ohne allgemeine Hochschulreife? Wie Herkunft, Bildungsverlauf und Wahlmotive den Studienerfolg beeinflussen. Marburg: Tectum Wissenschaftsverlag.

GEFORDERT VOM

Bundesministerium

für Bildung

und Forschung 


\section{Entwicklung beruflicher Schulen im regionalen Verbund}

Marc Casper, Bernadette Dilger, Frederik Fischer, Katharina Fütterer, Nicole Naeve-Stoß und Tade Tramm

\section{Einleitung}

Berufliche Schulen entwickeln sich in Zusammenarbeit in regionalen Bildungsnetzwerken. Das Spektrum reicht von verpflichtender Zusammenarbeit bis $\mathrm{zu}$ projektbasierter, freiwilliger Kooperation. Ziel des Beitrages ist es, Chancen und Herausforderungen von Schulentwicklung (SE) an beruflichen Schulen in Verbünden anhand von zwei Projekten aufzuzeigen. Dazu werden in Anlehnung an das Drei-Wege-Modell der Schulentwicklung nach Rolff (1998) die Ebenen der pädagogisch-didaktischen, der personellen sowie der organisatorisch-institutionellen SE als Aufgabenfeld der Berufs- und Wirtschaftspädagogik thematisiert und die Dimensionen der SE im Verbund im Vergleich zu den SE-Dimensionen der Einzelschule erweitert.

Die kooperativ-schulübergreifende und curricular-didaktische SE wird aus „KaBueNet“, dem Netzwerk der Berliner Oberstufenzentren zur Entwicklung des Curriculums für den/die Kaufmann/-frau für Büromanagement, vorgestellt. Der Zusammenhang von profilierter regionaler Bildungsangebotsgestaltung und institutioneller Entwicklung von Verbünden wird am Beispiel des „Regionalen Berufsbildungszentrums (RBZ) Dortmund“ verdeutlicht. Die Gegenüberstellung und Analyse der beiden Projekte erfolgt u. a. unter Bezugnahme auf Aspekte der Educational Governance mit Blick auf Motive und Impulse für die Projektinitiative, die Konstellationen sowie die Chancen, Herausforderungen und Grenzen der Verbundarbeit.

\section{Das Projekt „KaBueNet“}

\subsection{Motivation und Ausgangslage}

Zum Schuljahr 2014 wurde der neue Ausbildungsberuf ,Kaufmann-/frau für Büromanagement" eingeführt, in dem sich die vorigen bürowirtschaftlichen Berufe vereinen. Mit einem Jahr Vorlauf begannen sieben Berliner Oberstu- 
fenzentren unter wissenschaftlicher Begleitung mit der Entwicklung eines gemeinsamen kompetenzorientierten Lehrplans für den Berufsschulunterricht. Dieser regionale Verbund namens „KaBueNet“ (vgl. www.kabuenet. de) stellt sich dabei mehreren Herausforderungen (vgl. Casper 2016, 3f.):

1. Die Bewältigung der Neuordnung ist das vorrangige Problem aus der Sicht der Lehrenden, die inhaltlich und pragmatisch für den laufenden Unterricht handlungsfähig sein wollen.

2. Die Einführung des Lernfeldkonzepts ist für alle Beteiligten eine zusätzliche Herausforderung, da der neue Beruf erstmals nach Lernfeldern statt nach Fächern gegliedert ist. Die Interpretationsnotwendigkeit des neuen, offeneren Rahmenlehrplans ermöglicht eine eigenständigere curriculare Entwicklung auf Seiten der Schulen, fordert diese aber eben auch ein. Von den Kollegien an den einzelnen Schulen wird eine erhöhte curricular-didaktische Kompetenz gefordert, die eine „Neudefinition didaktischer Arbeit“ (Sloane 2002, 10) darstellt. Diese Kompetenzen können aus dem Kontext traditioneller Lehrarbeit heraus nicht selbstverständlich als vorhanden angenommen werden (vgl. Tramm \& Naeve-Stoß 2016, 50; Straßer 2013, 497).

3. Die Entwicklung einer Kooperationskultur ergibt sich als dritte Problemebene. Schulen lassen sich traditionell eher als „lose gekoppelte Systeme“ (Bormann 2000, 3) kennzeichnen. Im Fachunterricht können Lehrende als Experten eines Fachs relativ autonom unterrichten, wohingegen im fachübergreifenden Lernfeldunterricht eine höhere Abstimmung zwischen den Beteiligten notwendig ist. Das Lehrerteam wird im Lernfeldkonzept zur zentralen Instanz (vgl. Casper 2016, Straßer 2013, Sloane 2003).

In diesem Sinne können die genannten Herausforderungen im Rahmen der Neuordnung auch als (1) inhaltliche, (2) didaktisch/konzeptionelle sowie (3) soziale/organisationale Reformdimensionen interpretiert werden. Um diesen Herausforderungen zu begegnen, bietet sich bei Stadtstaaten wie Berlin eine schulübergreifende Kooperation an, als Ergänzung zur schulinternen Kooperation.

\subsection{Organisation und Arbeitsstränge}

Um als Verbund aufzutreten, sind neben gemeinsamen Zielen auch gemeinsame Beschlüsse und ein gewisses Maß an Verbindlichkeit notwendig. Dennoch hat ein Netzwerk wie KaBueNet größere Freiheitsgrade als eine einzelne, hierarchisch organisierte schulische Institution. Nach den Kategorien der (Educational) Governance-Literatur lässt sich KaBueNet als Netzwerk zwischen den Idealtypen Gemeinschaft und Organisation bezeichnen. Für Ka- 
BueNet ist ,auf der schulübergreifenden Ebene durchaus eine realtypische Gemeinschaft zu erkennen [...], die auf der Reziprozität der aufgeteilten Lernfeldentwicklung basiert (dem Austausch von zeitversetzt und arbeitsteilig erstellten Produkten)“ (Casper 2016, 18). Die Arbeitsteilung erfolgt entlang der in Abbildung 1 dargestellten Arbeitsstränge.

\section{Abb. 1: Aufbauorganisation in KaBueNet}

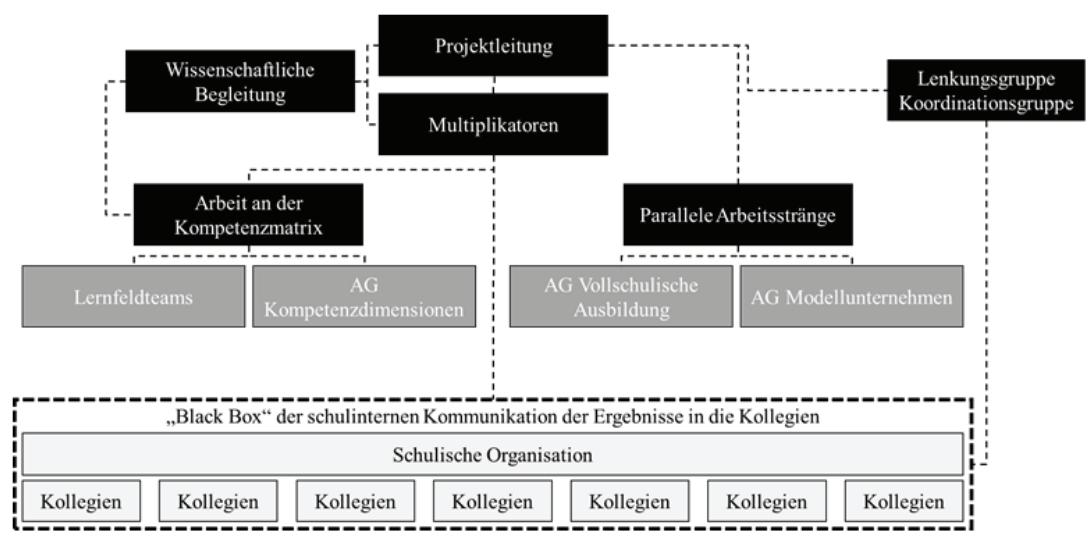

Quelle: Casper $(2016,19)$

Zentraler Bezug der curricularen Entwicklung ist eine Kompetenzmatrix im Sinne des Hamburger Modells. Gemeinsam verabschiedete Gestaltungskriterien sowie Planungsformate bieten somit zwar Orientierung und einen verbindlichen Rahmen, inhaltlich unterliegt die curriculare Arbeit der einzelnen Gruppen jedoch einer projekttypischen Ergebnisoffenheit. Die insgesamt 13 Lernfelder des Bildungsgangs wurden auf die beteiligten Schulen verteilt, sodass im Lernfeld-Arbeitsstrang schulinterne Teams je ein bis drei Lernfelder auf mehreren Planungsebenen ausarbeiten. Eine weitere AG mit jeweils einem Vertreter pro Schule erarbeitet parallel ein System von Kompetenzdimensionen, das als bildungsgangübergreifendes Ordnungsraster zur Entwicklung beruflicher Handlungskompetenz dienen soll. Die beiden Achsen Lernfelder und Kompetenzdimensionen bilden schließlich die Kompetenzmatrix als curriculares Kerninstrument (linker Zweig in Abbildung 1). Parallel hierzu arbeiten schulübergreifende Gruppen an der Ausarbeitung der vollschulischen Variante des Bildungsgangs und an der Entwicklung eines authentischen Modellunternehmens als Lernumwelt (rechter Zweig in Abbildung 1).

Bei der schulübergreifenden Entwicklungsarbeit handelt es sich also um einen Austausch auf Vertrauensbasis, da keine Sanktionen formalisiert wurden und es in KaBueNet keine disziplinierende hierarchische Instanz gibt. Qualitätsambitionen und Erwartungshaltungen können sich allein auf die 
Selbstverpflichtung und das Vertrauen der Beteiligten berufen, was den oben erwähnten Realtypus der Gemeinschaft unterstreicht. Hier zeigt sich ein besonderes Potenzial von Netzwerken für kooperative Bildungsverantwortung, denn ,schwache Verbindungen erhöhen die Wahrscheinlichkeit der Koordination zwischen Akteuren, die sich in Situationen mit einem höheren Anforderungsgehalt nicht aufeinander abgestimmt hätten bzw. gar nicht erst aufeinander getroffen wären" (Kuper 2004, 243-244). Um dieses Aufeinandertreffen zu institutionalisieren und dem Netzwerk eine Projektstruktur zu geben, entwickelte die wissenschaftliche Begleitung ein Workshop-Konzept, in dessen Rahmen bislang 40 schulübergreifende Workshops stattfanden (Stand 31.03.2017). Diese Workshops wurden zu Beginn maßgeblich von der wissenschaftlichen Begleitung gestaltet und sukzessive in die Eigenverantwortung der Projektschulen übertragen, sodass eine langfristige, kontinuierliche Selbststeuerung angestrebt wird.

Auf der Ebene der einzelnen Schulen wiederum entzieht sich das Meiste bislang der Beobachtung der wissenschaftlichen Begleitung (vgl. „Black Box" in der Grafik). Es ist zu erwarten, dass der Erfolg der Implementation an den einzelnen Schulen in besonderem Maße von schulspezifischen organisationalen Faktoren abhängt. Hierauf zielt die Implementationsforschung und -begleitung ab, die aktuell für die kommende Projektphase konzipiert wird.

\subsection{Die Hamburger Kompetenzmatrix als Impulsgeber für Personalentwicklung}

Anhand des Drei-Wege-Modells der Schulentwicklung (vgl. Rolff 1998, 305) wird deutlich, dass Unterrichtsentwicklung (UE), Organisationsentwicklung $(O E)$ und Personalentwicklung (PE) konsequenterweise zusammen zu denken sind. Ausgangspunkt in KaBueNet ist die curriculare Entwicklung von Lernfeldunterricht, das zentrale Anliegen ist also eine Entwicklung des Unterrichts. Hieraus ergeben sich Konsequenzen für die Organisation und das Personal.

Die grundlegende Annahme des Hamburger Modells ist, dass die Ausarbeitung der Lernfelder für ein kompetenzorientiertes Curriculum allein nicht ausreicht. Bei einem rein lernfeldorientierten Lehrplan würden zwar prozessbezogene Kenntnisse, Fertigkeiten und Fähigkeiten ausgebildet. Eine ganzheitliche Entwicklung beruflicher Kompetenzen über die Lernfelder hinweg würde jedoch vernachlässigt, wenn Lernfelder für sich isoliert betrachtet werden. Es bedarf also einer zweiten entwicklungslogischen Achse, die quer $\mathrm{zu}$ allen Lernfeldern mitgedacht werden muss, einer Struktur von Kompetenzdimensionen, die sich aus dem Kompetenzverständnis der Kultusministerkonferenz (KMK-Rahmenlehrpläne) bzw. dem Kompetenzbegriff nach Roth ableitet. Die Dimensionen der „Sach-, Sozial- und Werteinsicht“ (Roth 
1971, 596) bzw. der Fach-, Sozial- und Selbstkompetenz (KMK) müssen für den konkreten Beruf ausdifferenziert und spezifiziert werden. Dies ist eine Reflexion und Zielklärung, die von den Entwicklern und unterrichtenden Kollegien im Rahmen einer schulnahen Curriculumentwicklung geleistet werden muss. Im Fall KaBueNet geschieht dies in der erwähnten schulübergreifenden Arbeitsgruppe „Kompetenzdimensionen“.

\subsection{Zukünftige Projektaufgaben und Herausforderungen}

Zum Sommer 2017 ist der erste Jahrgang beendet, der auf der Basis einer ersten Version der Kompetenzmatrix ausgebildet wurde. Im nächsten Schritt verlagert sich die Netzwerkarbeit somit auf die Evaluation und Überarbeitung auf Basis des bisherigen Unterrichts sowie auf Implementation und Transfer des Curriculums. Aus Sicht der wissenschaftlichen Begleitung ergibt sich der folgende Ausblick für die drei Wege der Schulentwicklung:

- In der Dimension Unterrichtsentwicklung: Iterative Weiterentwicklung und Umsetzung von Lernsituationen im Sinne der Kompetenzmatrix;

- In der Dimension Organisationsentwicklung: Implementierung, Dissemination und Qualitätssicherung in den Einzelschulen;

- In der Dimension Personalentwicklung: Weiterbildung und Coaching der Kollegien an den Einzelschulen durch ausgebildete Multiplikatoren.

\section{Das Projekt „RBZ Dortmund“}

\subsection{Motivation und Ausgangslage}

Das Projekt „Regionales Berufsbildungszentrum (RBZ) Dortmund“ wird in Zusammenarbeit der acht städtischen Berufskollegs, der Stadt Dortmund und des Ministeriums für Schule und Weiterbildung (MSW) des Landes NRW durchgeführt. Im Zeitraum von 2013 bis 2018 wird die Entwicklung und der Aufbau eines Kooperationsverbundes der acht Berufskollegs in verschiedenen Handlungsbereichen der beruflichen Bildung bearbeitet (vgl. Buschfeld, Dilger, Fischer \& Fütterer 2016). Impuls und Auslöser war die Absicht, einen institutionellen Rahmen zu schaffen, um Herausforderungen wie dem grundlegenden Strukturwandel, der demografischen Entwicklung und dem zukünftigen Fachkräftebedarf zu begegnen. Dieser Rahmen sollte dabei der Bedeutung der beruflichen Schule als regionaler Standortfaktor und den besonderen sozioökonomischen Bedingungen einer Großstadt gerecht werden. Intention des Projektvorhabens ist die Entwicklung des strategischen Bildungsange- 
bots, die Entwicklung der institutionellen Rahmenbedingungen sowie einer nachhaltigen Verbund-Arbeit. Die einzelnen Akteure haben unterschiedliche Motivationen, um sich in dem Projekt zu engagieren:

- Durch das Projekt entstehen für das einzelne Berufskolleg Möglichkeiten der Schulentwicklung und der Professionalisierung der eigenen Lehrkräfte.

- Die Gesamtheit der Berufskollegs (als Berufskolleg-Verbund) profitiert durch das Projekt von der Konzentration auf Kooperation (statt auf Konkurrenz), einer effizienten Ressourcennutzung, der Sicherung und Stärkung des Einflusses in der Region (durch das Sprechen mit „einer Stimme") sowie durch die kooperative Lösung dynamischer Anforderungen.

- Die Vorteile für die Stadt Dortmund als Kommune ergeben sich durch eine gemeinsam koordinierte Berufskollegentwicklungsplanung, die Klärung und Zentralisierung von Ansprechpartnern in den Berufskollegs sowie durch ein effizientes Ressourcenmanagement (insb. in Hinblick auf Räumlichkeiten).

- Durch das Projekt werden Alternativen für die Zusammenarbeit beruflicher Schulen entwickelt. Das Projekt stellt somit ein Modellvorhaben für das Land NRW (in Gestalt des Ministeriums für Schule und Weiterbildung) dar.

\subsection{Handlungsfelder des Projekts}

Die Arbeit der einzelnen Projektgruppen ist in Qualitätszirkeln (QZ) organisiert, die sich in vier Handlungsfeldern ordnen. Diese gliedern sich in zwei strategische und zwei operative Handlungsfelder, die sich in ihren Leitideen einerseits mit dem Ziel, Pläne für ein zukünftiges RBZ zu konzipieren, und andererseits bereits jetzt Prozesse unabhängig von der Form des späteren RBZs zu erproben, unterscheiden (siehe Abbildung 2).

Auf der strategischen Ebene werden Konzepte zur Gestaltung, Planung und Steuerung des regionalen Bildungsangebotes entwickelt. Dies führt von der Planung und Umsetzung von Prozessen eines kommunalen Bildungsmonitorings als Datenbasis (QZ Monitoring) zu einer abgestimmten regionalen Bildungsangebotsplanung (QZ regionales Bildungsangebot). Das Handlungsfeld stellt gleichzeitig die Schnittstelle zur Regionalen Berufskollegentwicklungsplanung der Stadt Dortmund (BK-EP) dar. Die BK-EP möchte neben einer Analyse der Ist-Situation und einer Bedarfsabschätzung auf Grundlage der Entwicklung der Schülerzahlen einen Prozess zur Abstimmung und Gestaltung des regionalen Berufsbildungsangebots im Verbund implementieren (vgl. Fischer \& Göckede 2015). In Handlungsfeld II werden Szenarien für eine institutionelle Ausgestaltung der Zusammenarbeit der acht Berufskollegs erarbeitet, mit der Frage, in welcher institutionell-organisato- 
rischen Form das RBZ agieren soll (vgl. Bücking \& Dilger 2015). Es wurden drei Szenarien entwickelt, die sich in ihren jeweiligen Mehrwerten für verschiedene Zielgruppen, ihrem Institutionalisierungsgrad, dem zugrunde liegenden Steuerungsgedanken sowie der Akteurskonstellation unterscheiden: 1) RBZ als Dienstleistungszentrum, 2) RBZ als Modellberufskolleg und 3) RBZ als Dachverband.

Abb. 2: Handlungsfelder des Projekts RBZ Dortmund

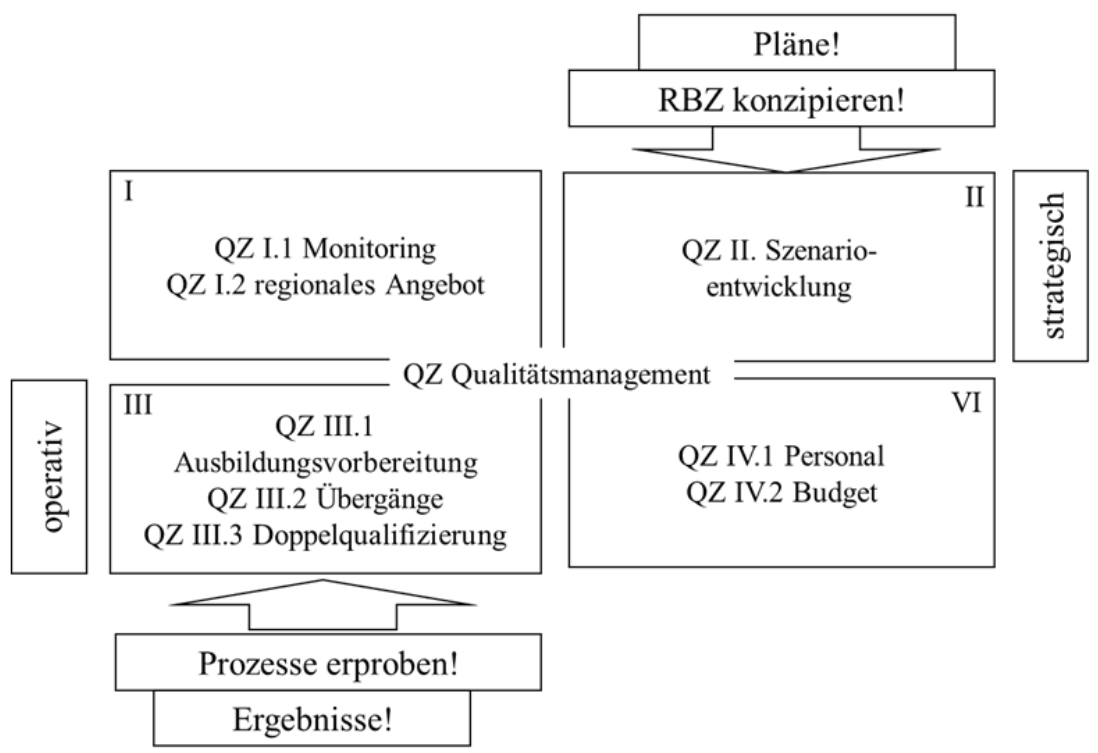

Quelle: Eigene Darstellung nach Buschfeld \& Dilger (2014, 23)

Auf der operativen Ebene werden konkrete schüler- und unterrichtsnahe Prozesse in Zusammenarbeit entwickelt und erprobt (z. B. in der Ausbildungsvorbereitung, der Doppelqualifizierung und der Eingangs- und Übergangsberatung). Es werden dabei Bildungsangebote berücksichtigt, die im Verbund mit Mehrwert entwickelt und umgesetzt werden können. Handlungsfeld IV beschäftigt sich mit der Abstimmung und Erprobung von Personalmanagement- und Beschaffungsprozessen im Verbund. Durch die starke Abhängigkeit der inhaltlichen Ausgestaltung von den institutionellen und rechtlichen Rahmenbedingungen wurde die Arbeit in die Entwicklungsworkshops des Handlungsfeldes II integriert (vgl. Buschfeld \& Dilger 2016, 7). Der QZ Qualitätsmanagement versteht sich als Dienstleister und An- 
sprechpartner für alle Projektteilnehmer und Qualitätszirkel und ist daher keinem Handlungsfeld direkt zugeordnet.

\subsection{Bisherige wissenschaftliche Projektbegleitung und-erfahrung}

Als theoretisch-analytische Referenzkonzepte dienen die Arbeiten zur Schulentwicklung (vgl. Rolff, Buhren, Lindau-Bank \& Müller 2000), zu Qualitätsmanagement beruflicher Schulen (vgl. Dubs 2003) und zu Verbundstrukturen und Educational Governance (vgl. Altrichter, Brüsemeister \& Wissinger 2007; Maag Merki, Langer \& Altrichter 2014). Über die Aktivitäten in den einzelnen Projektjahren wird jährlich ein Bericht der wissenschaftlichen Begleitung veröffentlicht (vgl. Buschfeld \& Dilger 2014; Buschfeld \& Dilger 2015; Buschfeld \& Dilger 2016).

Im Projekt sind verschiedene Spannungsfelder erkennbar geworden. Das Projekt steht auf der strategischen Ebene vor der Herausforderung, dass die Schulen ihre Rolle als alleinstehende Institution verlassen müssen und gefordert sind, eine gemeinsame strategische Zielrichtung zu definieren. Dazu gehört bspw. der Abgleich von Mehrwerten und Aufwänden, die mit dem Verbund für die einzelne Schule verknüpft sind. Auf der operativen Ebene werden die konkreten Herausforderungen der Zusammenarbeit sichtbar, die wiederum im Rahmen der strategischen Ausrichtung zu berücksichtigen sind. Es muss entschieden werden, in welchem Maß Entscheidungsbefugnisse von der Einzelschule an einen Verbund abgegeben werden und wie ein Verbund bei der Steuerung einer Einzelschule mitwirken darf.

\section{Entwicklung beruflicher Schulen im Verbund - eine typisierende Sichtweise}

\subsection{Zur Orientierung}

Beide dargestellten Projekte können in der Tradition einer wirtschaftspädagogischen Gestaltungsforschung gesehen werden, bei der ein Problem der (wirtschaftspädagogischen) Praxis den Ausgangspunkt bildet. Das Erkenntnisinteresse ist dabei von Beginn an auf die systematische Analyse des Problems ausgerichtet, um auf der Grundlage eine theoriegeleitete Weiterentwicklung der Praxis anzuregen (vgl. Sloane 2005; Euler 2011; Dilger 2014). Im Folgenden sollen anhand einer kategorialen Gegenüberstellung der beiden Projekte Gemeinsamkeiten und Unterschiede herausgearbeitet werden, um anschließend auf der Basis der bisherigen Erfahrungen durch die wissen- 
schaftliche Begleitung Herausforderungen, aber auch die Potentiale einer Schulentwicklung im Verbund zu skizzieren.

\subsection{Impulse für die berufliche Schulentwicklung im Verbund}

Herausforderungen für die Entwicklung von beruflichen Schulen im Verbund lassen sich nach exogenen Einflüssen und endogenen Impulsen unterscheiden: Für die beiden skizzierten Projekte können sowohl exogene als auch endogene Entwicklungsimpulse für die berufliche Schulentwicklung im Verbund herausgestellt werden. Während es beim RBZ-Projekt insbesondere um die Institutionalisierung und Professionalisierung der schulischen Organisationsstrukturen und -prozesse geht, wurde das Projekt KaBueNet durch die curriculare Reform in den Büroberufen initiiert. Bei beiden Projekten zeigt sich jedoch das Zusammenspiel der eher pädagogisch-didaktischen mit den organisatorisch-institutionellen Entwicklungsimpulsen. Berufliche Schulentwicklung im Verbund erfolgt demnach in den Bereichen „Entwicklung einer professionellen Bildungsinstitution“ und „Entwicklung profilierter Bildungsangebote".

\subsection{Zielsetzungen der beruflichen Schulentwicklung im Verbund}

Basierend auf den bisherigen Erfahrungen können bei den Zielsetzungen vier unterschiedliche Potentiale und deren Nutzen für die Verbünde identifiziert werden:

- Lern-, Entwicklungs- und Innovationspotentiale können sich daraus ergeben, dass vorhandene Expertisen zusammenkommen, gemeinsam genutzt und weiterentwickelt werden, was sich positiv auf die Qualität von Innovationen auswirken kann - ganz im Sinne: Gemeinsam sind die Schulen entwicklungsfähiger, adaptiver und innovativer als allein.

- Promotorenpotential kann dadurch entstehen, dass Interessen gebündelt und mit Blick auf strategische Ziele zusammen verfolgt werden können.

- Effizienzpotential ist anhand von Synergieeffekten erkennbar, wobei hier vor allem die Optimierung des Ressourceneinsatzes als Mehrwert angesehen werden kann.

- Aus den drei vorgenannten Mehrwerten kann sich im Idealfall ein Autonomiepotential ergeben, denn durch die Arbeit im Verbund können erweiterte Handlungsspielräume des Verbundes entstehen. Neue Innovationen, in Verbindung mit einer Bündelung der Interessen und des Durchsetzungswillens sowie der Optimierungsabsicht bei den eingesetzten Ressourcen, können zu veränderten Rahmenbedingungen 
für die Handlungs- und Gestaltungsspielräume des gesamten Verbunds und/oder auch der Einzelschule führen.

Unserer Erfahrung nach stehen bei den Projekten die Lern-, Entwicklungsund Innovationspotentiale im Vordergrund, gefolgt von den Effizienzpotentialen und den Promotionspotentialen. In der Diskussion im Innenverhältnis der Verbünde hat das Autonomiepotential einen großen Stellenwert. In der Außendarstellung und damit in der Legitimierung der Projekte wird der Mehrwert der gemeinsamen Entwicklung und der damit möglichen Innovationen herausgestellt.

\subsection{Schulentwicklungsdimensionen als Ansatzpunkte für die Klassifikation von Entwicklung beruflicher Schulen im Verbund}

Ausgehend vom Drei-Wege-Modell der Schulentwicklung geht es darum, Unterrichtsentwicklung, Organisationsentwicklung und Personalentwicklung zusammen zu denken (vgl. Rolff 1998, 305). Ein Vergleich der beiden Projekte zeigt, dass im Berliner Projekt die Unterrichtsentwicklung den Ausgangspunkt der Verbundarbeit dargestellt hat und sich daraus Konsequenzen und Entwicklungsherausforderungen für die Dimensionen Personal und Organisation ergeben haben. Im Projekt „RBZ Dortmund“ wurden hingegen im Sinne einer integrierten institutionellen Entwicklung alle drei Entwicklungsdimensionen von Beginn an gleichzeitig bearbeitet. Während sich also beim KaBueNet-Projekt die Frage nach den erforderlichen Entwicklungen auf der Personal- und Organisationsebene zur Sicherstellung der Implementation und Dissemination der curricularen Produkte stellt, entsteht beim RBZ-Projekt die Herausforderung, die unterschiedlichen Entwicklungsaktivitäten auf allen drei Ebenen aufeinander zu beziehen und zusammen zu führen.

\subsection{Merkmale der Educational Governance als Klassifikationspunkte von Entwicklung beruflicher Schulen im Verbund}

Mit der Perspektive der Bildungssteuerung gelangen die Merkmale der Akteure und ihrer Konstellation, die Entwicklung der Mehrebenenlogik zur Steuerung von Bildungsinstitutionen sowie die Formen der kooperativen Arbeitsweise und die Struktur der Arbeitsweise in den Blick (vgl. Kussau \& Brüsemeister 2007). Durch die Form der Zusammenarbeit in beiden Projekten wird eine neue Akteurskonstellation geschaffen, nämlich der Verbund, was $\mathrm{zu}$ einer veränderten Zuweisung von Rechten und Leistungsstrukturen führt. Im RBZ-Projekt stellt sich diesbezüglich als zentrale Frage, wie die Entscheidungsstrukturen im Verbund aufgestellt werden und wie diese mit dem bisher bestehenden Leitungssystem der einzelnen Schule in Abstim- 
mung gebracht werden können. Im Projekt KaBueNet ergeben sich u. a. Fragestellungen hinsichtlich der Verbindlichkeit der entwickelten curricularen Produkte für die Einzelschulen, des Einbezugs und der Beteiligung der Kolleginnen und Kollegen, die nicht am Entwicklungsprozess beteiligt waren und letztlich auch hinsichtlich der Übertragung der Entwicklungsstrategie auf weitere Bildungsgänge.

Mit der Zusammenschließung zu Verbünden bekommt in beiden Projekten der Verbund als Institution eine höhere Bedeutung für die Gestaltung des regionalen Bildungsangebots. Durch die gemeinsame Ausgestaltung und die Etablierung des Verbundes als eigene Akteurkonstellation wird eine weitere Ebene in der Steuerung des regionalen Bildungsangebotes eingeführt. Dies eröffnet einerseits Möglichkeiten für eine spezifischere Profilbildung in der Region und es verbessert die Chancen zur Interessensbündelung und -durchsetzung durch das Wirken des Verbundes als kollektiver Akteur. Andererseits entstehen dadurch Veränderungen in der Abstimmung zwischen den Akteuren in der Berufsbildungsregion. Zudem entsteht als neue Entwicklungsherausforderung die nachhaltige Gestaltung der Beziehungen innerhalb des Verbundes.

\subsection{Zusammenfassende Überlegungen, Potentiale und Herausforderungen der beruflichen Schulentwicklung im Verbund}

In Erweiterung des Drei-Wege-Modells nach Rolff (1998) ergeben sich u.E. angesichts der beruflichen Schulentwicklung in Verbünden folgende drei Dimensionen:

- Entwicklung curricular-didaktischer Konzeptionen,

- Entwicklung eines regional-profilierten Bildungsangebotes,

- Entwicklung von Strukturen und Prozessen mit personellen und institutionellen Regelungen und Kompetenzen im Innen- und Außenverhältnis des Verbundes.

Aus den bisherigen Erfahrungen der wissenschaftlichen Begleitungen in den Projekten können die folgenden Herausforderungen für die Arbeit in regionalen Verbünden festgehalten werden:

- Schaffung einer Balance zwischen gemeinsamen und konkurrierenden Aufgaben sowie Aushandlung von gemeinsamen Interessen und den Interessen der Einzelschulen;

- Sicherstellung der Dissemination und Implementation der im Verbund entwickelten Produkte an den jeweiligen Einzelschulen;

- Motivation der Einzelakteure zur Beteiligung am Verbund, insbesondere dann, wenn der Mehrwert bzw. das Potential der Verbundarbeit nicht 
unmittelbar für den einzelnen Akteur „erfahrbar“ wird, sondern andere Akteure im Verbund entlastet. Und schließlich die

- Entwicklung nachhaltiger Netzwerkstrukturen und -prozesse. In diesem Zusammenhang stellt sich vor allem der hohe Koordinationsaufwand als eine Herausforderung dar, da die Arbeit im Verbund u. a. ein hohes personelles Engagement sowie zeitliche Ressourcen erfordert.

Im Sinne einer ersten Bilanzierung können anhand der bisherigen Erfahrungen folgende Mehrwerte der Arbeit in regionalen Verbünden angenommen werden:

- Größen- und Effizienzeffekte: Durch Entwicklungsarbeiten im berufsschulischen Verbund entsteht die Möglichkeit, Bildungsangebote in der Region zu sichern. Zudem können Effizienzeffekte durch die gemeinsame Entwicklungsarbeit entstehen, insbesondere kann ein Potential bei der didaktischen Konzeptionsentwicklung und in der Etablierung von schülernahen Prozessen festgestellt werden (z. B. bei der Entwicklung doppelqualifizierender Angebote in der dualen Ausbildung).

- Innovationseffekte können durch gemeinsam entwickelte Produkte (z. B. schulische Curricula, Unterrichtsmaterialien) und/oder didaktische Prozessanleitungen (z. B. Handreichungen zur Planung eines lernfeldorientierten Curriculums) entstehen, aber beispielweise auch durch neu konzipierte Bildungsgänge, die an mehreren Einzelschulen angeboten werden.

- Möglichkeiten für Qualitätseffekte ergeben sich durch kollegiale Diskussionen, da ein Austausch z. B. über „gute“ Schule, ,guten“ Unterricht usw. stattfinden und darüber eine Verständigung auf gemeinsame Qualitätskriterien erfolgen kann.

\section{Literatur}

Altrichter, H., Brüsemeister, T. \& Wissinger, J. (2007). Educational Governance: Handlungskoordination und Steuerung im Bildungssystem. Wiesbaden: VS.

Bormann, I. (2000). Schule als lernende Organisation - Kann eine veränderte Lehrerbildung Schule verändern? Sowi-online. Online: http://www.sowi-online.de/sites/ default/files/bormann.pdf (05.01.2017).

Bücking, K. \& Dilger, B. (2015). Entwicklung von regionalen Verbund-Szenarien für berufliche Schulen. Kölner Zeitschrift für Wirtschaft und Pädagogik, 30(59), 2550 .

Buschfeld, D. \& Dilger, B. (2014, 2015, 2016). Weiterentwicklung der acht Berufskollegs in städtischer Trägerschaft zu einem Regionalen Berufsbildungszentrum: Jahresberichte der wissenschaftlichen Begleitung. Online: http://www.rbzdo.de/wissenschaftliche-begleitung (16.12.2016). 
Buschfeld, D., Dilger, B., Fischer, F. \& Fütterer, K. (2016). Weiterentwicklung der Berufskollegs im Verbund - ein Bericht über das Modellprojekt „Regionales Berufsbildungszentrum Dortmund“. bwp@Berufs-und Wirtschaftspädagogik-online, 31, 1-19. Online: http://www.bwpat.de/ausgabe31/buschfeld_etal_bwpat 31.pdf (12.12.2016).

Casper, M. (2016). Schulübergreifende kooperative Curriculumentwicklung aus der Perspektive von organisationalem Lernen, Change Management und Educational Governance am Beispiel des Netzwerks KaBueNet für den Beruf „Kaufmann/frau für Büromanagement".bwp@Berufs- und Wirtschaftspädagogik-online, 31, 1-26. Online: http://www.bwpat.de/ausgabe31/casper_bwpat31.pdf (05.01. 2017).

Dilger, B. (2014). Herausforderungen der Gestaltungsforschung in der Wirtschaftspädagogik. In U. Braukmann, B. Dilger \& H.-H. Kremer (Hrsg.), Wirtschaftspädagogische Handlungsfelder. Festschrift für Peter F. E. Sloane zum 60. Geburtstag, (S. 363-378). Detmold: Eusl.

Dubs, R. (2003). Qualitätsmanagement für Schulen. Studien und Berichte des IWP, 13. St. Gallen: IWP, Institut für Wirtschaftspädagogik.

Euler, D. (2011). Wirkung- vs. Gestaltungsforschung - eine feindliche Koexistenz. Zeitschrift für Berufs- und Wirtschaftspädagogik, 107(4), 520-542.

Fischer, F. \& Göckede, B. (2015). Regionale Berufskollegentwicklungsplanung als schwierig-vereinfachtes Verfahren. Kölner Zeitschrift für Wirtschaft und Pädagogik, 30(59), 3-24.

Kuper, H. (2004). Netzwerke als Form pädagogischer Institutionen. In W. Böttcher \& E. Terhart (Hrsg.), Organisationstheorie in pädagogischen Feldern. Analyse und Gestaltung, (S. 237-252). Wiesbaden: VS Verlag für Sozialwissenschaften.

Kussau, J. \& Brüsemeister, T. (2007). Educational Governance: Zur Analyse der Handlungskoordination im Mehrebenensystem der Schule. In H. Altrichter, T. Brüsemeister \& J. Wissinger (Hrsg.), Educational Governance. Handlungskoordination und Steuerung im Bildungssystem, (S. 14-53). Wiesbaden: Springer VS.

Maag Merki, K., Langer, R. \& Altrichter H. (2014). Educational Governance als Forschungsperspektive: Strategien, Methoden, Ansätze. Wiesbaden: Springer VS.

Rolff, H.-G. (1998). Entwicklung von Einzelschulen: Viel Praxis, wenig Theorie und kaum Forschung - Ein Versuch, Schulentwicklung zu systematisieren. In H.-G. Rolff, K. O. Bauer, K. Klemm \& H. Pfeiffer (Hrsg.), Jahrbuch der Schulentwicklung. Daten, Beispiele und Perspektiven, (S. 295-326). Weinheim: Beltz.

Rolff, H.-G., Buhren, C. G., Lindau-Bank, D. \& Müller, S. (2000). Manual Schulentwicklung: Handlungskonzept zur pädagogischen Schulentwicklungsberatung (SchuB). Weinheim, Basel: Beltz.

Roth, H. (1971). Pädagogische Anthropologie. Hannover: Schroedel.

Sloane, P. F. E. (2002). Schulorganisation und schulische Curriculumarbeit. In R. Bader \& P. F. E. Sloane (Hrsg.), Bildungsmanagement im Lernfeldkonzept. Curriculare und organisatorische Entwicklung. Beiträge aus den Modellversuchsverbünden NELE \& SELUBA, (S. 9-29). Paderborn: Eusl-Verlag.

Sloane, P. F. E. (2003). Schulnahe Curriculumentwicklung. bwp@,Berufs- und Wirtschaftspädagogik - online, 4, 1-23. Online: http://www.bwpat.de/ausgabe4/ sloane_bwpat4.pdf, (05.01.2017). 
Sloane, P. F. E. (2006). Berufsbildungsforschung. In R. Arnold und A. Lipsmeier (Hrsg.), Handbuch der Berufsbildung, (S. 610-627). Wiesbaden: VS Verlag für Sozialwissenschaften.

Straßer, P. (2013). Lernfelder - die (ungenutzte) Wiederkehr des Exemplarischen. Zeitschrift für Berufs- und Wirtschaftspädagogik, 109(4), 496-510.

Tramm, T. \& Naeve-Stoß, N. (2016). Lernfeldübergreifende Kompetenzentwicklung als curriculare Planungsperspektive im Kontext einer kooperativen Curriculumentwicklung in der kaufmännischen Berufsbildung. In A. Dietzen, R. Nickolaus, B. Rammstedt \& R. Weiß (Hrsg.), Kompetenzorientierung. Berufliche Kompetenzen entwickeln, messen und anerkennen, (S. 49-70). Bielefeld: W. Bertelsmann Verlag. 


\section{Herausgeberschaft}

Prof. Dr. Susan Seeber

Georg-August-Universität Göttingen

Professur für Wirtschaftspädagogik und Personalentwicklung

Susan.seeber@wiwi.uni-goettingen.de

Prof. Dr. Jürgen Seifried

Universität Mannheim

Lehrstuhl für Wirtschaftspädagogik - Berufliches Lehren und Lernen

seifried@bwl.uni-mannheim.de

Prof. Dr. Birgit Ziegler

Technische Universität Darmstadt

Institut für Allgemeine Pädagogik und Berufspädagogik

ziegler@bpaed.tu-darmstadt.de

\section{Autorinnen und Autoren}

Prof. Dr. Karin Büchter

Helmut Schmidt Univeristät Hamburg

Fakultät für Geistes- und Sozialwissenschaften

Berufsbildung

buechter@hsu-hh.de

Marc Casper, M. Ed.

Fakultät für Erziehungswissenschaft

Institut für Berufs- und Wirtschaftspädagogik

Universität Hamburg

marc.casper@uni-hamburg.de

Prof. Dr. Bernadette Dilger

Universität St. Gallen

Institut für Wirtschaftspädagogik

bernadette.dilger@unisg.ch 
Dr. Katja Driesel-Lange

Westfälische Wilhelms-Universität Münster

Institut für Erziehungswissenschaft

Arbeitsbereich: Berufspädagogik

katja.driesel-lange@uni-muenster.de

Frederik Fischer, Dipl.-Hdl.

Universität zu Köln

Institut für Berufs-, Wirtschafts- und Sozialpädagogik

frederik.fischer@uni-koeln.de

Prof. Dr. Dietmar Frommberger

Universität Osnabrück

Berufs- und Wirtschaftspädagogik

dietmar.frommberger@uni-osnabrueck.de

Prof. Dr. Bärbel Fürstenau

Technische Universität Dresden

Lehrstuhl Wirtschaftspädagogik

baerbel.fuerstenau@tu-dresden.de

Katharina Fütterer, M. Sc.

Universität zu Köln

Institut für Berufs-, Wirtschafts- und Sozialpädagogik

katharina.fuetterer@uni-koeln.de

Ass.-Prof. Mag. Dr. Christoph Helm

Johannes Kepler Universität Linz

Abteilung für Bildungsforschung

christoph.helm@jku.at

Dr. Mandy Hommel

Technische Universität Dresden

Lehrstuhl Wirtschaftspädagogik

mandy.hommel@tu-dresden.de

Heike Jahncke, B. A., M. Sc.

Carl von Ossietzky Universität Oldenburg

Fachgebiet Berufs- und Wirtschaftspädagogik

heike.jahncke@uni-oldenburg.de 
Karina Kiepe, B. A., M. Ed. Helmut Schmidt Universität Berufs- und Arbeitspädagogik kiepek@hsu-hh.de

Mag. Bettina Kreuzer Johannes Kepler Universität Linz Abteilung für Bildungsforschung kreuzer.bettina@gmx.net

Silke Lange, B. Sc., M. Ed.

Universität Osnabrück

Berufs- und Wirtschaftspädagogik

silke.lange@uni-osnabrueck.de

Prof. Dr. Anke Langner

Technische Universität Dresden

Professur für Erziehungswissenschaft mit dem Schwerpunkt Inklusive Bildung anke.langner@tu-dresden.de

Prof. Dr. Claudia Leopold

Universität Freiburg

Zentrum für Lehrerinnen- und Lehrerbildung

claudia.leopold@unifr.ch

Prof. Mario López

Universität Santiago de Chile

Facultad de Ingeniería

Departamento de Ingeniería Industrial

mario.lopez@usach.cl

Prof. Dr. Nicole Naeve-Stoß

Universität zu Köln

Institut für Berufs-, Wirtschafts- und Sozialpädagogik

nicole.naeve-stoss@uni-koeln.de

Mag. Jacqueline Netzthaler

Johannes Kepler Universität Linz

Abteilung für Bildungsforschung

jacqueline.netzthaler@gmx.at 
Prof. Dr. Manuela Niethammer

Technische Universität Dresden

Professur Bautechnik, Holztechnik, Farbtechnik und

Raumgestaltung/Berufliche Didaktik

manuela.niethammer@tu-dresden.de

Svenja Ohlemann, Dipl.-Betriebswirtin (FH)

Technische Universität Berlin

Institut für Erziehungswissenschaft

Fachgebiet Pädagogische Psychologie

svenja.ohlemann@tu-berlin.de

Prof. Héctor Ponce

Universität Santiago de Chile

Facultad de Administración y Economia

hector.ponce@usach.cl

Prof. Dr. Andreas Rausch

Universität Mannheim

Professur für Berufs- und Wirtschaftspädagogik

rausch@uni-mannheim.de

Prof. i.V. Dr. Christian Schmidt

Universität Kassel

Institut für Berufsbildung

Fachgebiet Berufs- und Wirtschaftspädagogik

christian.schmidt@uni-kassel.de

Dr. Matthias Söll

Universität Kassel

Institut für Berufsbildung

Fachgebiet Wirtschaftsdidaktik

matthias.soell@uni-kassel.de

Prof. Dr. Tade Tramm

Fakultät für Erziehungswissenschaft

Institut für Berufs- und Wirtschaftspädagogik

Universität Hamburg

tade.tramm@uni-hamburg.de 
Dr. Julia Warwas

Otto-Friedrich-Universität Bamberg

Lehrstuhl für Wirtschaftspädagogik

julia.warwas@uni-bamberg.de 


\section{Schlüssel- kompetenzen}

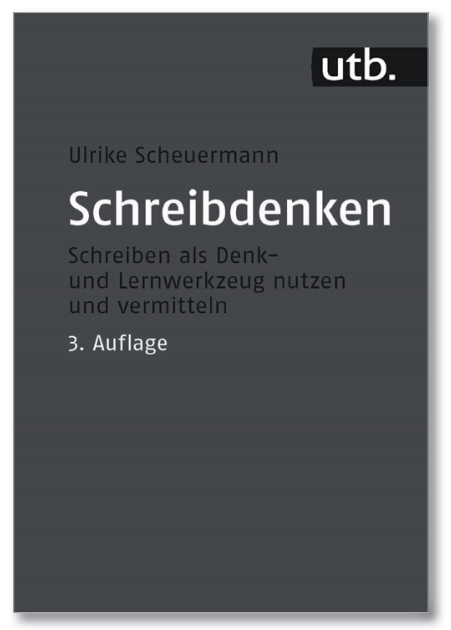

Ulrike Scheuermann

Schreibdenken

Schreiben als Denk- und Lern-

werkzeug nutzen und vermitteln

utb S

Kompetent lehren, Band 3

3., durchgesehene Auflage 2016.

128 S. Kt. $9,99 €(D), 10,30 €(A)$

ISBN 978-3-8252-4717-1

eISBN 978-3-8385-4717-6

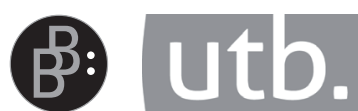

\section{utb.}

\section{Schreibmythen} entzaubern

Ungehindert schreiben

in der Wissenschaft

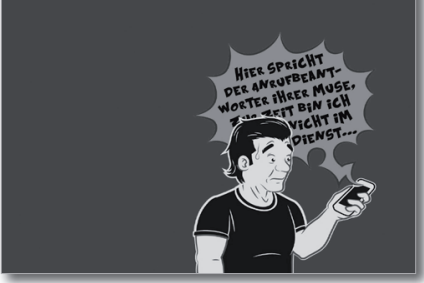

Christian Wymann

Schreibmythen

entzaubern

Ungehindert schreiben in der

Wissenschaft

utb S

2016. 118 S. Kt.

$12,99 €(D), 13,40 €(A)$

ISBN 978-3-8252-4660-0

eISBN 978-3-8385-4660-5

Jetzt in Ihrer Buchhandlung bestellen oder direkt bei: www.utb-shop.de utb GmbH I Industriestr. 2 I 70565 Stuttgart 


\section{Schreiben, Publizieren, Präsentieren}

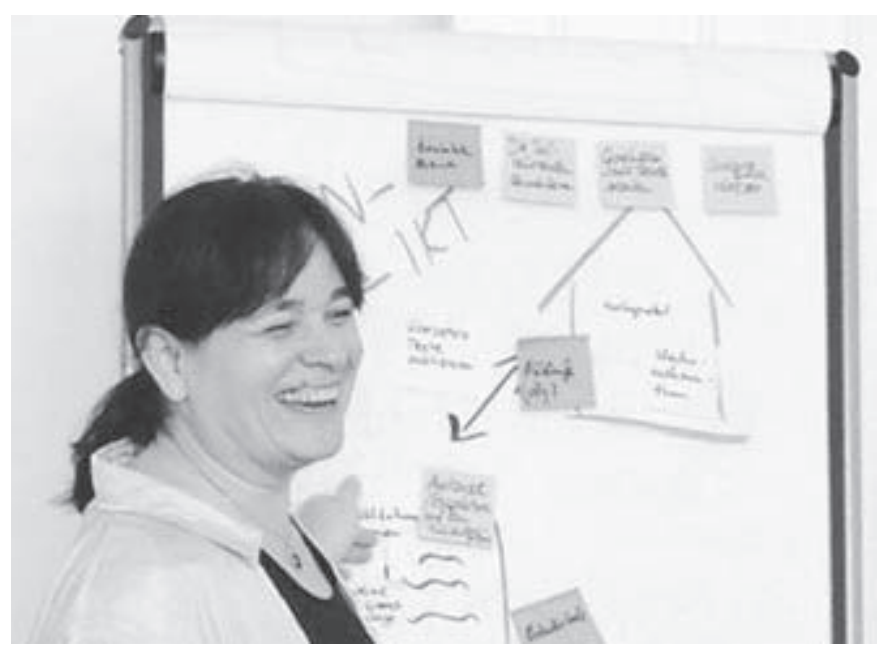

budrich training

bietet Schulungen für Studierende und AkademikerInnen in den Schlüsselkompetenzen der wissenschaftlichen Kommunikation - auf Deutsch und auf Englisch.

Workshops, Vorträge, Seminare:

- Wissenschaftliches Schreiben

- Wissenschaftliches Publizieren

- Präsentieren und Moderieren

Wir bieten auch Einzelberatungen und Coachings.

budrich training - Schlüsselkompetenzen in der Wissenschaft

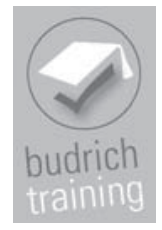

Wir freuen uns auf Ihre Anfrage:

budrich training

Stauffenbergstr. 7. D-51379 Leverkusen Opladen

Tel +49 (0)2171.344.594 • Fax +49 (0)2171.344.693•

info@budrich.de

www.budrich-academic.de • www.budrich-training.de 


\section{Interpretative Schul- und Unterrichtsforschung}
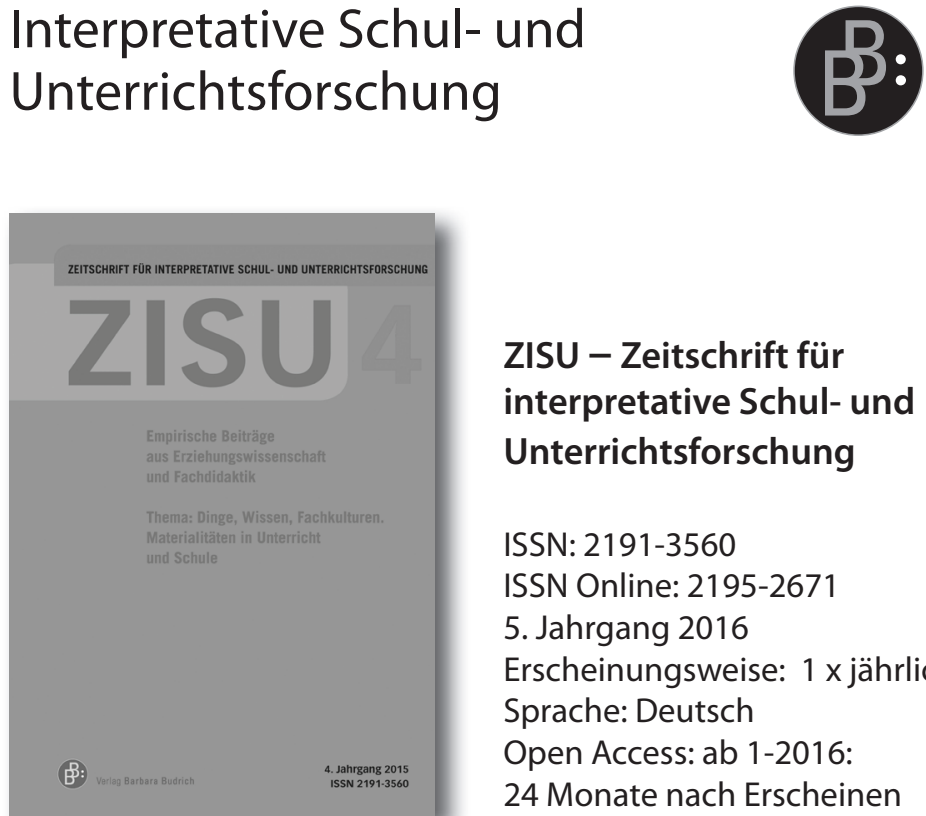

ZISU - Zeitschrift für interpretative Schul- und Unterrichtsforschung

ISSN: 2191-3560

ISSN Online: 2195-2671

5. Jahrgang 2016

Erscheinungsweise: 1 x jährlich Sprache: Deutsch

Open Access: ab 1-2016:

24 Monate nach Erscheinen

Die ZISU ist ein Forum für

Redaktion: herausragende qualitativProf. Dr. Torsten Pflugmacher empirische Forschungsergebnisse aus Fachdidaktik und Erziehungswissenschaft. Sie dient der Vernetzung von gemeinsamen Forschungsperspektiven und will die Aufmerksamkeit auf interdisziplinäre, theoriefähige, qualitative Schul- und Unterrichtsforschung richten.

Didaktik der Deutschen Sprache und Literatur Seminar für deutsche Philologie Universität Göttingen

Waldweg 26

37073 Göttingen

zisu@budrich-journals.de

Weitere Informationen zur Zeitschrift sowie Bestellmöglichkeiten unter:

\section{zisu.budrich-journals.de}

